\title{
La sectorización basada en criterios energéticos como herramienta para la gestión hídrica de redes de distribución de agua
}

Francisco Javier Salguero Barceló

Directores:

Ricardo Cobacho Jordán

Miguel Ángel Pardo Picazo

Tesis Doctoral

Valencia, octubre 2021 


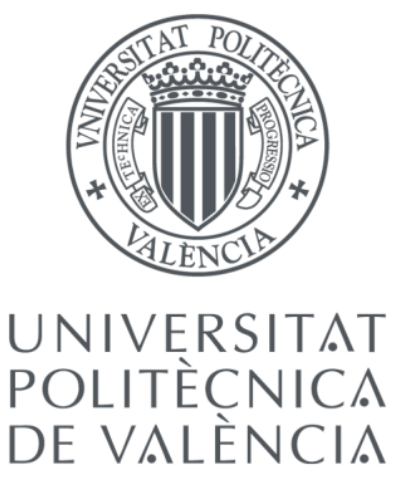

\section{La sectorización basada en criterios energéticos como herramienta para la gestión hídrica de redes de distribución de agua}

Presentada por:

Francisco Javier Salguero Barceló

Dirigida por:

Ricardo Cobacho Jordán Miguel Ángel Pardo Picazo 


\section{Agradecimientos}

No podría concluir este trabajo sin mostrar mi más sincero agradecimiento a todas aquellas personas que, de una manera u otra, han hecho que esto sea posible.

En primer lugar, a mis directores, Ricardo Cobacho Jordán y Miguel Ángel Pardo Picazo, quienes me han guiado y aconsejado ante las innumerables dudas. Su experiencia, cercanía y amabilidad constantes durante todos estos años me han permitido sentirme cómodo y seguro durante todo el camino.

A María José y a Ana, mi mujer y mi hija, por sus sonrisas incondicionales durante todo el proceso y por todo el tiempo sacrificado para que este trabajo saliera adelante. A mis padres y abuelos, quienes me lo han dado todo sin pedir nada a cambio. A mis hermanos y a mis amigos, por compartir cada victoria y alegría.

A mis compañeros y amigos de Aguas de Valencia por brindarme la oportunidad de disfrutar trabajando.

Y en último lugar, a todos aquellos que piensan que las cosas no son posibles. Realmente ellos mueven el mundo, al impulsar a quienes creemos lo contrario.

Sin todos ellos, este trabajo no habría sido posible. 


\section{Resumen}

La sectorización de las redes ha demostrado ser una metodología estratégica en la reducción de los volúmenes incontrolados fugados, considerado uno de los principales problemas a los que tienen que enfrentarse diariamente los gestores de los sistemas de distribución de agua. Su principal objetivo es facilitar la monitorización de las redes, dividiéndolas en subsistemas, sobre los que se puede realizar un mejor y más fácil control de las anomalías en el consumo y presión.

Uno de los inconvenientes que presenta esta técnica recae en su diseño. No existe una solución única, sino que atiende a diversos criterios para realizarlo. De manera generalizada, este diseño se basa en la experiencia del propio diseñador, siguiendo criterios puramente prácticos y con procesos manuales de prueba y error, cuyo grado de dificultad está vinculado al tamaño y complejidad de la propia red.

Esta tesis plantea una metodología sistemática para el diseño de la sectorización de una red de distribución de agua, según criterios energéticos, con el fin de facilitar la delimitación de cada uno de los sectores de la red. Como resultado, se obtiene una organización de la red en sectores buscando una optimización energética de la misma, de tal modo que posteriores técnicas de mejora, como es el caso de la gestión de presiones, se vean favorecidas.

La sectorización ha resultado ser una técnica eficaz en la búsqueda de fugas. Sin embargo, como supone una pérdida de capacidad hidráulica y una merma en la calidad del agua, esta tesis se cuestiona el planteamiento de una técnica no intrusiva alternativa que logre los mismos resultados. Así, se propone el desarrollo de una metodología que localice las fugas centrada en la interdependencia de todos los elementos y en el empleo de sensores de caudal y presión.

El trabajo pone de manifiesto que es posible sectorizar la red de tal manera que se consigan mayores beneficios para la misma. Ahora bien, también es posible el empleo de otras técnicas que no requieran el cierre de tuberías pero que desprendan soluciones similares. El empleo de una técnica u otra dependerá de cada caso, de los objetivos establecidos y de la política de trabajo de cada gestor. 


\section{Resum}

La sectorització de les xarxes ha demostrat ser una metodologia estratègica en la reducció dels volums incontrolats perduts, considerat un dels principals problemes als quals han d'enfrontar-se diàriament els gestors dels sistemes de distribució d'aigua. El seu principal objectiu és facilitar la monitorització de les xarxes, dividint-les en subsistemes, sobre els quals es pot realitzar un millor i més fàcil control de les anomalies en el consum i pressió.

Un dels inconvenients que presenta aquesta tècnica recau en el seu disseny. No existeix una solució única, sinó que atén diversos criteris per a realitzar-ho. De manera generalitzada, aquest disseny es basa en l'experiència del propi dissenyador, seguint criteris purament pràctics i amb processos manuals de prova i error, el grau de dificultat del qual està vinculat a la grandària i complexitat de la pròpia xarxa.

Aquesta tesi planteja una metodologia sistemàtica per al disseny de la sectorització d'una xarxa de distribució d'aigua, segons criteris energètics, amb la finalitat de facilitar la delimitació de cadascun dels sectors de la xarxa. Com a resultat, s'obté una organització de la xarxa en sectors buscant una optimització energètica d'aquesta, de tal manera que posteriors tècniques de millora, com és el cas de la gestió de pressions, es vegen afavorides.

La sectorització ha resultat ser una tècnica eficaç en la cerca de fuites d'aigua. No obstant això, com suposa una pèrdua de capacitat hidràulica i una disminució en la qualitat de l'aigua, aquesta tesi es qüestiona el plantejament d'una tècnica no intrusiva alternativa que aconseguisca els mateixos resultats. Així, es proposa el desenvolupament d'una metodologia que localitze les fuites d'aigua centrada en la interdependència de tots els elements i en l'ús de sensors de cabal i pressió.

El treball posa de manifest que és possible sectoritzar la xarxa de tal manera que s'aconseguisquen majors beneficis per a aquesta. Ara bé, també és possible l'ús d'altres tècniques que no requerisquen el tancament de canonades però que desprenguen solucions similars. L'ús d'una tècnica o una altra dependrà de cada cas, dels objectius establits i de la política de treball de cada gestor. 


\section{Abstract}

The sectorization of networks has proven to be a strategic methodology in the reduction of uncontrolled leakage volumes, considered one of the main problems that water distribution system managers must face on a daily basis. Its main objective is to facilitate the monitoring of the networks, dividing them into subsystems, on which a better and easier control of anomalies in consumption and pressure can be performed.

One of the drawbacks of this technique lies in its design. There is no single solution, but it is based on different criteria. In general, this design is based on the designer's own experience, following purely practical criteria and with manual trial and error processes, whose degree of difficulty is linked to the size and complexity of the network itself.

This thesis proposes a systematic methodology for the design of the sectorization of a water distribution network, according to energy criteria, in order to facilitate the delimitation of each of the sectors of the network. As a result, an organization of the network in sectors is obtained, looking for an energetic optimization of the same, in such a way that later improvement techniques, such as pressure management, are favoured.

Sectorization has proven to be an effective technique in the search for leaks. However, as it implies a loss of hydraulic capacity and a decrease in water quality, this thesis questions the approach of an alternative non-intrusive technique that achieves the same results. Thus, it is proposed the development of a methodology to locate the leaks focused on the interdependence of all the elements and the use of flow and pressure sensors.

The present work shows that it is possible to sectorize the network in such a way as to achieve greater benefits for the network. However, it is also possible to use other techniques that do not require pipe closure but provide similar solutions. The use of one technique or another will depend on each case, on the established objectives and on the working policy of each manager. 


\section{Tabla de contenidos}

Capítulo 1 Introducción y objetivos.......................................................................

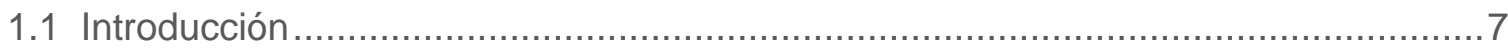

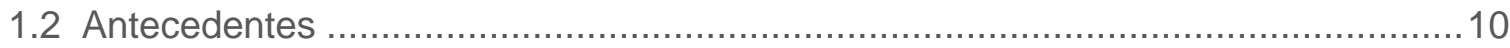

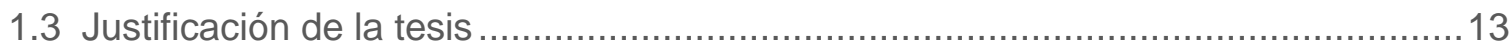

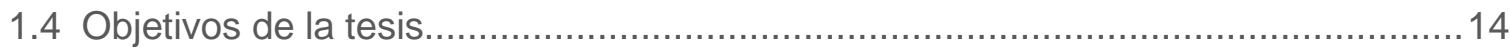

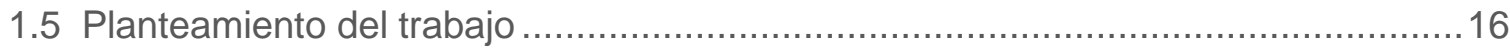

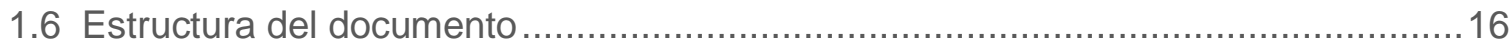

1.7 Marco colaborativo: Doctorado Industrial ...................................................... 17

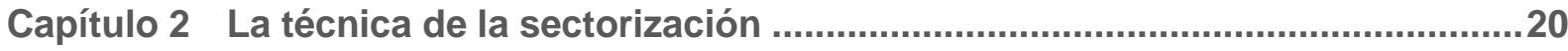

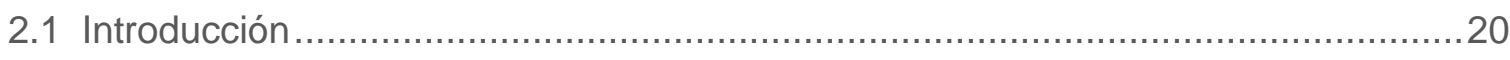

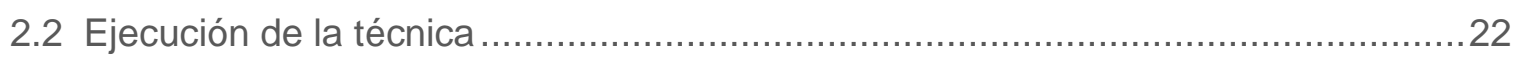

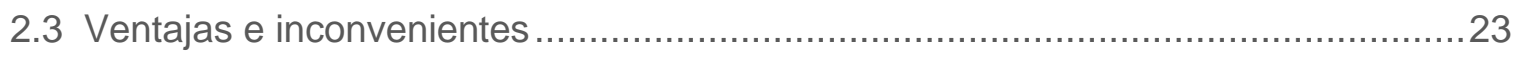

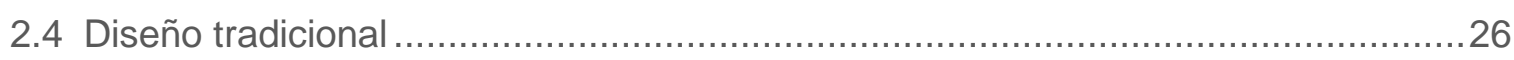

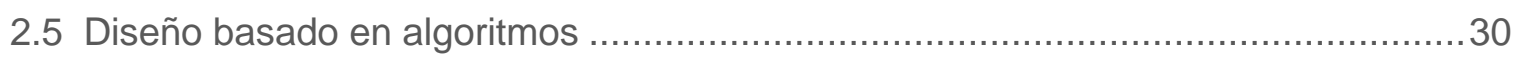

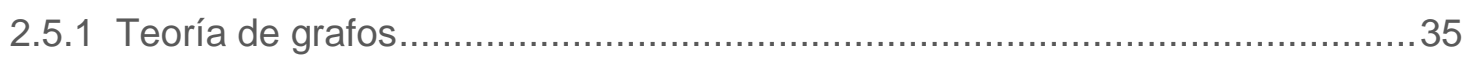

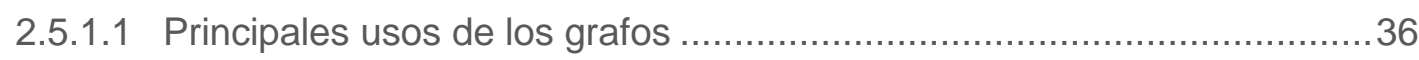

2.5.1.2 Aplicación en la sectorización de redes de distribución de agua..............37

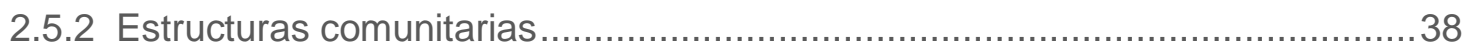

2.5.2.1 Principales usos de las estructuras comunitarias ..................................40

2.5.2.2 Aplicación en la sectorización de redes ............................................. 41

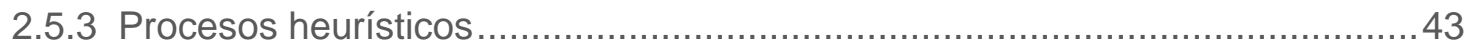

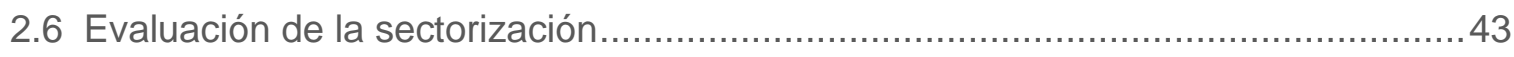

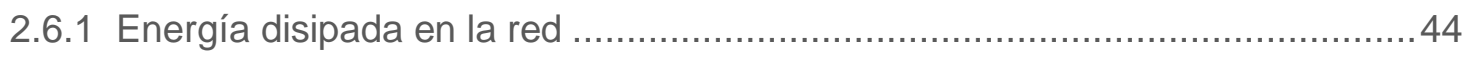




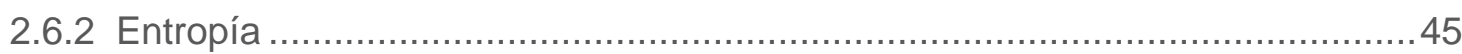

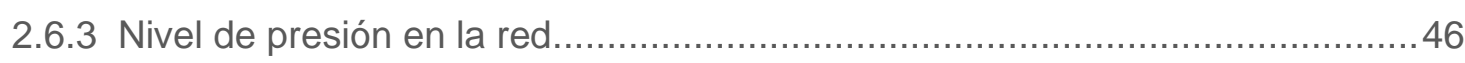

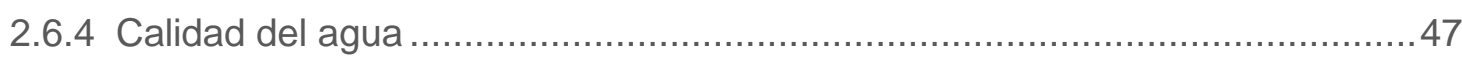

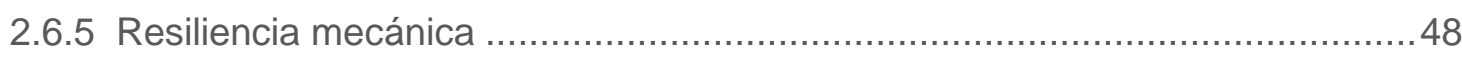

2.6.6 Capacidad de la red ante condiciones de incendio.......................................48

Capítulo 3 Aplicación de conceptos energéticos en la fase de diseño .......................50

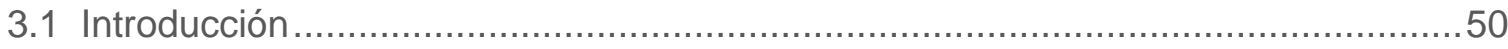

3.2 El concepto energético en los sistemas de distribución ....................................... 51

3.3 Diseño de sectorizaciones basado en conceptos energéticos ..............................54

3.3.1 La sectorización desde la óptica monocriterio (energética) …........................55

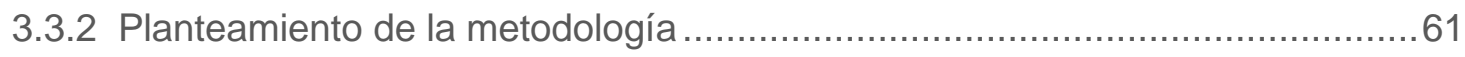

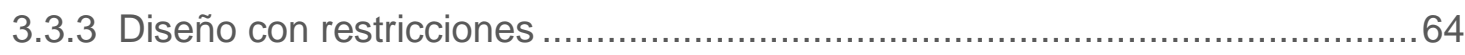

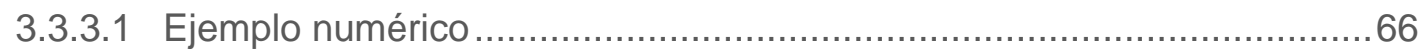

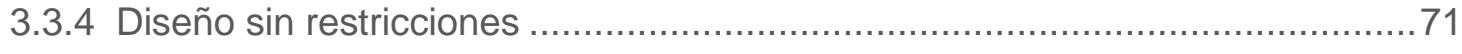

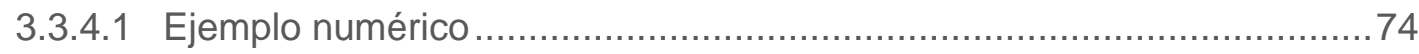

Capítulo 4 Sensores para la localización de fugas....................................................77

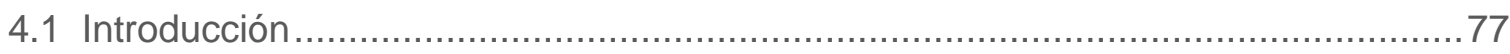

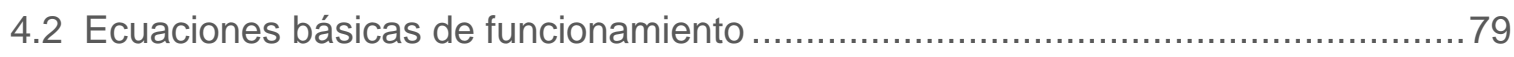

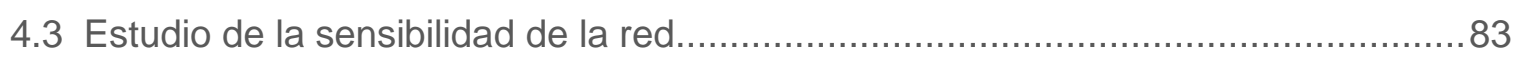

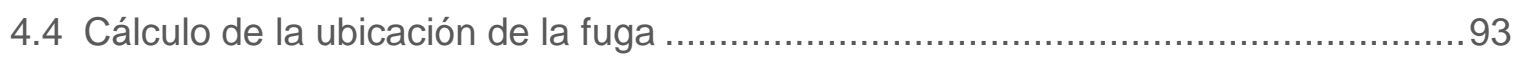

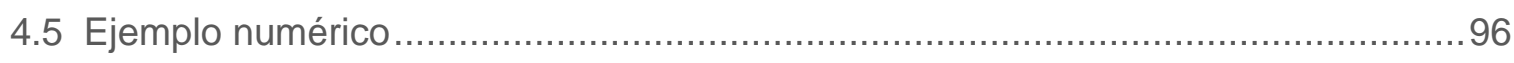

Capítulo 5 Casos de estudio ............................................................................... 108

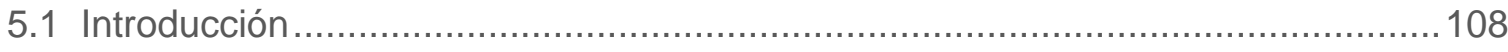

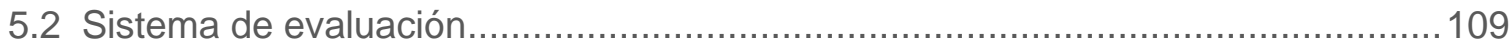

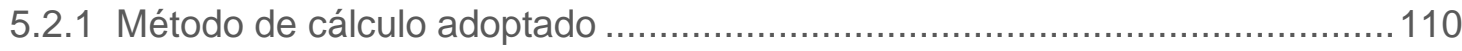

5.2.2 Objetivos de evaluación seleccionados..................................................111

5.2.3 Criterios de evaluación seleccionados ....................................................112

5.2.4 Métricas de evaluación seleccionadas ..................................................113

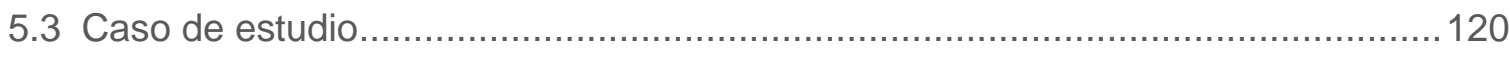

5.3.1 Caso práctico 1. Red sintética ......................................................... 120

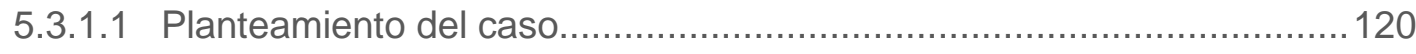

5.3.1.2 Sectorización propuesta según conceptos energéticos........................ 122

5.3.1.3 Sensorización para la localización de fugas........................................ 125

5.3.1.4 Efectividad y eficiencia en la búsqueda de fugas no declaradas...........131

5.3.1.5 Capacidad hidráulica de la red resultante .......................................... 133

5.3.1.6 Calidad del agua de la red resultante........................................... 135

5.3.1.7 Coste de implantación ................................................................. 136 
5.3.1.8 Valoración de las alternativas ...................................................... 137

5.3.1.9 Discusión de los resultados obtenidos ................................................ 138

5.3.2 Caso práctico 2. Red de distribución real ................................................... 141

5.3.2.1 Planteamiento del caso.............................................................. 141

5.3.2.2 Sectorización según conceptos energéticos ........................................ 145

5.3.2.3 Sensorización para la localización de fugas........................................ 150

5.3.2.4 Efectividad y eficiencia en la búsqueda de fugas no declaradas............156

5.3.2.5 Capacidad hidráulica de la red resultante .......................................... 157

5.3.2.6 Calidad del agua de la red resultante................................................ 158

5.3.2.7 Coste de implantación ................................................................ 159

5.3.2.8 Valoración de las alternativas ......................................................... 160

5.3.2.9 Discusión de los resultados obtenidos .............................................. 161

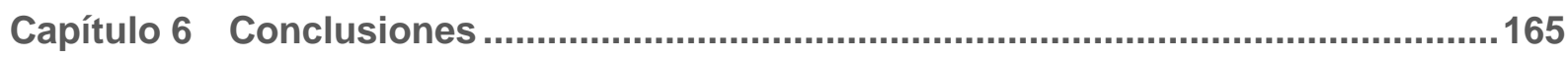

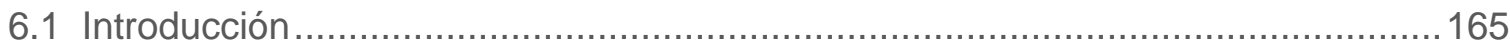

6.2 Criterios energéticos como guía en el diseño de la sectorización ......................... 166

6.3 Sensorización como herramienta para la localización de las fugas.......................167

6.4 Comparación de las metodologías............................................................... 168

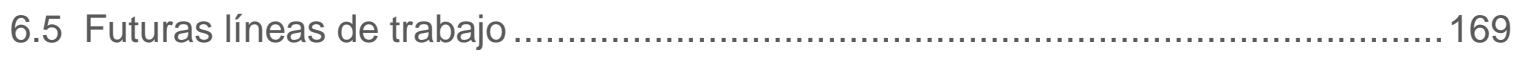

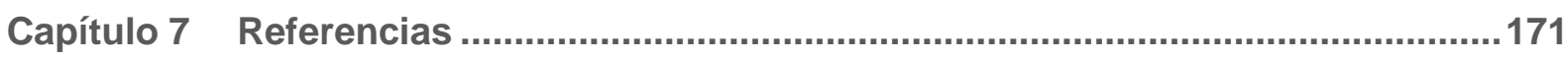




\section{Capítulo 1 Introducción y objetivos}

\subsection{Introducción}

El papel del agua en el desarrollo de la Humanidad es incuestionable. Desde su origen, el ser humano ha necesitado este preciado elemento en mayor o menor medida. En la Edad del Bronce, año 4.000 a.C., la escasez de precipitaciones en la antigua Mesopotamia convirtió a los ríos Tigris y Éufrates en la única manera de obtener agua. Este hecho, y la dependencia del regadío para los cultivos, obligaron a los agricultores asentados en las llanuras existentes entre ambos ríos a unirse, apareciendo así los primeros pueblos y ciudades (Viollet, 2010). Era tal la dependencia del agua con estas primeras civilizaciones, que los reyes y gobernantes veían en ella un arma de gran poder, de tal modo que, en caso de querer castigar a una determinada zona, tan solo tenían que cortarle el suministro. Con el paso de los años, el agua continuó ganando importancia hasta convertirse en un factor fundamental en el origen de las grandes capitales actuales. París, por ejemplo, fue fundada por los Parisios, entre los años 250 a.C. y 200 a.C. en lo que hoy se conoce como La Île de la Cité. Según señalan varios indicios, este lugar fue elegido por razones de defensa estratégica al estar protegidos por los brazos del rio Sena. Años después, concretamente en la mitad del siglo XV, y $1.600 \mathrm{~km}$ al sur, el agua sigue acompañando a la sociedad sevillana aportando, además de su valor intrínseco, un valor añadido. Así, el agua constituía uno de los más importantes atributos de prestigio y poder económico (Fernández, 2012). Aunque no es necesario echar la vista tan atrás para observar la importancia del agua en el desarrollo de la humanidad. Concretamente, el estado semiárido de Israel está viviendo, desde los años 1940, una auténtica explosión gracias, en parte, al agua (Siegel, 2017). Hoy en día, el agua continúa jugando un papel central en la economía, en el desarrollo de nuestras sociedades y en la forma que vemos el mundo. Sin embargo, el escenario futuro hacia el que nos dirigimos es bien distinto del pasado (WWAP, 2018). 
La mitad de la fuerza laboral mundial está concentrada en ocho sectores que depende del agua y de los recursos naturales: agricultura, silvicultura, pesca, energía, industria manufacturera con uso intensivo de recursos, reciclaje, construcción y transporte. Tan solo en la pesca, agricultura y silvicultura encuentran su empleo más de mil millones de personas. Y los cortes en los suministros de agua dulce amenazan seriamente a estos dos últimos sectores (OIT, 2013). Poniendo números a esta situación (OIT, 2015), se estima que 1.350 millones de puestos de trabajo (el $42 \%$ de la población activa total mundial) dependen fuertemente del agua. Por sectores, el $95 \%$ de los puestos de trabajo en el sector agrícola, el $30 \%$ en el sector industrial y el $10 \%$ en el sector de los servicios dependen directamente de este elemento. Del resto, el $36 \%$ de la población activa total mundial depende de una manera moderada del agua. Probablemente, tienen una dependencia moderada el $5 \%$ de los puestos de trabajo en el sector agrícola, el $60 \%$ en el sector industrial y el $30 \%$ en el sector de los. O, dicho de otro modo, el $78 \%$ de la fuerza laboral mundial dependen del agua.
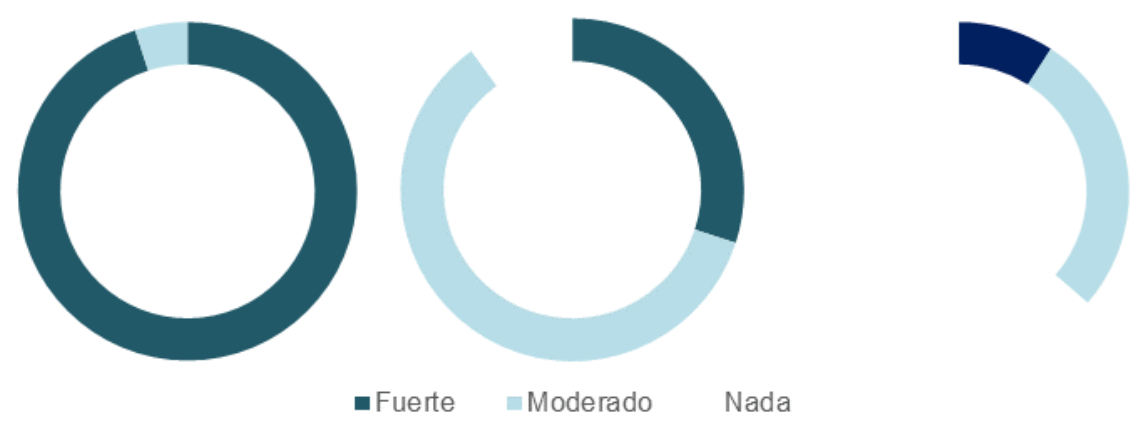

Figura 1.1. Nivel de dependencia con el agua de diferentes sectores (agricultura, industria y servicios)

Sobre todo, en el apoyo al sustento de los estratos más empobrecidos, la agricultura tiene un papel muy importante, donde representa el principal sector de empleo. Una buena muestra de ello es la situación del África subsahariana, donde este sector engloba el $60 \%$ de los puestos de trabajo. Evidentemente, la calidad y cantidad de empleo en el sector agroalimentario, la producción agrícola y la estabilidad de los ingresos se verán comprometidos por un suministro de agua insuficiente o irregular.

En el sector de la energía, la dependencia del agua no es nada despreciable, representando alrededor del $15 \%$ del total mundial de agua extraída. Aunque hay que destacar el auge en el uso de las energías renovables, como la solar fotovoltaica, la eólica y la geotérmica, las cuales no utilizan agua. Una gran parte de la producción total de electricidad depende estrechamente del agua y se estima que la demanda de este recurso aumentará de forma significativa, en más de un tercio en el periodo 2010-2035 (WWAP, 2014).

En el sector de la industria, grandes cantidades de trabajadores se concentran en los subsectores con mayor consumo de agua: 22 millones en alimentación y bebidas, 20 millones en el sector químico, farmacéutico, goma y neumáticos, así como 18 millones en la electrónica (OIT, 2015). Aproximadamente, el $4 \%$ de las extracciones de agua mundiales son empleadas en la industria. Y para el año 2050, se ha pronosticado en un $400 \%$ el incremento en el consumo de la industria manufacturera (OECD, 2012).

Al margen de la agricultura, la industria o la energía, el consumo de las redes municipales tampoco es nada despreciable, representando el $10 \%$ del consumo mundial de agua dulce (WWAP, 2012). 
Queda más que justificado que el agua es una componente esencial de las economías nacionales y locales y es necesario que las políticas sobre la gestión del agua tomen un mayor significado e importancia a nivel global. Pero son numerosas las amenazas a las que se debe enfrentar: el aumento del nivel de vida y una acelerada urbanización, el aumento de la demanda de agua, alimentos (especialmente carne) y energía debido a una población creciendo a una velocidad de vértigo (UNESCO, 2017; WWAP, 2019), un mundo cada vez más contaminado, unos recursos explotados limitados, unas infraestructuras ineficientes y en decadencia (Watson et al., 2004) y un cambio climático irremediable. Pero como bien indica Ban Ki-moon en el Informe de las Naciones Unidas sobre el Desarrollo de los Recursos Hídricos en el Mundo 2016 (WWAP, 2016), el agua queda en un segundo plano ante los problemas como desarrollo sostenible, las migraciones humanas, los conflictos internacionales o los desastres naturales. Dejando de lado los asuntos del agua, se corre el peligro de provocar un grave efecto negativo sobre los modos de vida, las poblaciones y la economía, con unos resultados potencialmente catastróficos y considerablemente costosos. Es posible que las oportunidades de crecimiento se vean limitadas por la escasez de agua, y no solo esto. En áreas como la agricultura, los asentamientos humanos, la industria, la producción de energía y el mantenimiento de ecosistemas, se intensificará todavía más la lucha por el agua entre los usuarios (OECD, 2012). Esto perjudicará a la seguridad alimentaria, la energía y las aguas regionales, y potencialmente a la seguridad geopolítica, provocando migraciones a varias escalas.

Por lo tanto, la gestión sostenible del agua y sus infraestructuras y el acceso a un suministro seguro, fiable y asequible es la única vía de escape. Se conseguirá así mejorar el nivel de vida, una mayor inclusión social, expandir las economías locales y promover la creación de puestos de trabajo más dignos. Principalmente en países en vías de desarrollo.

En esta línea de la sostenibilidad y volviendo al 10\% del consumo mundial de agua dulce producido en las ciudades, cabe destacar que no todo el volumen inyectado en los sistemas tiene como destino el consumo final, sino que un alto porcentaje se pierde por el camino. Este fenómeno, el agua que se escapa del sistema sin ser controlada, es conocido coloquialmente como fugas (Ofwat, 2008) y en conjunto volumen incontrolado fugado (Lambert \& Hirner, 2000). Respecto a su magnitud, la Environmental Protection Agency (EPA, 2010) realizó un estudio que mostraba la tasa de fugas en distintas ciudades norteamericanas. En todas ellas, las fugas oscilaban entre el 35,15\% de Williamsport (Pennsylvania) y el 15,20\% de Memphis (Tennessee). Pero, ampliando el rango geográfico, este porcentaje puede sobrepasar el $50 \%$ como en Bytom, Polonia (Wojciech \& Krzysztof, 2012), o en países del tercer mundo (ADB, 2008; Kingdom et al., 2006). A escala nacional, las fugas en España suman el $25 \%$ del volumen anual suministrado en las redes de abastecimiento (INE, 2018).

La detección y reducción de los consumos incontrolados fugados son, por tanto, uno de los mayores retos de los gestores de las redes de distribución de agua a presión. De manera individual, una fuga ocasionada en cualquier elemento de la infraestructura ajena a la propiedad individual puede provocar daños materiales, como son los producidos por las inundaciones ocasionadas por una rotura, por ejemplo. Desde la óptica de la infraestructura, un volumen incontrolado implica la necesidad de un mayor volumen producido, y con ello un mayor consumo energético, de productos químicos para potabilizar y mayores infraestructuras. Y a escala global, un mayor volumen producido significa una mayor cantidad de $\mathrm{CO}_{2}$ emitido a la atmósfera o un agravante para el fenómeno de la sequía, entre otros. 
Y aunque la minimización de las fugas es casi una tarea obligatoria y justificada, y la gestión de estas ha sido abordada por infinidad de estudios (Renaud et al., 2107), su consecución es un trabajo arduo y nada trivial. Por un lado, los sistemas de distribución de agua, centrándonos en las zonas urbanas, se han transformado en grandes infraestructuras complejas compuestas por tuberías de todos los calibres y materiales, innumerables nudos de consumo y varios puntos de regulación, constituyendo un sistema, cuyo punto de funcionamiento varía según lo hace las demandas. Y por otro, la tasa de renovación de las infraestructuras es menor que la tasa de envejecimiento con lo que aumenta la probabilidad de aparición de fugas y roturas.

En conclusión, el camino a recorrer por los gestores de los sistemas de distribución de agua está bien marcado. Tienen la obligación de realizar una gestión sostenible y óptima de todos los recursos, con especial relevancia el agua. Y encuentran en la reducción de volúmenes fugados una importante oportunidad. Evidentemente, dicha oportunidad de mejora requerirá un trabajo extenso y, a la vez, especializado.

\subsection{Antecedentes}

No todo el volumen inyectado en un sistema de distribución tiene como destino final el consumo por parte del usuario, sino que porcentajes importantes de este volumen se escapan del sistema sin ser consumidos por el usuario final. Esto se debe a que la presurización del dota al fluido de una gran facilidad para escapar por cualquier orificio que encuentro a su paso (huecos, roturas, desgastes en los materiales, etc.). Cuando esto se produce, todo el volumen que se escapa del sistema sin ser controlado y de manera fortuita recibe el nombre volumen incontrolado fugado (Lambert \& Hirner, 2000), pérdidas reales (Farley \& Trow, 2003) o, más coloquialmente, fugas (Ofwat, 2008).

Estos volúmenes incontrolados fugados representan uno de los principales problemas a los que tienen que enfrentarse los gestores de los sistemas de distribución de agua. Y es un fenómeno presente en todos los sistemas, en mayor o menor medida, según sus características o política de gestión seguida. La aparición de las roturas supone un tema muy estudiado por investigadores y gestores de redes de abastecimiento. Se puede afirmar que dependen de muchos factores, pero los más representativos son la edad del material, las variaciones de presión que sufre la tubería, el número de acometidas, la longitud y el diámetro. Los volúmenes incontrolados fugados no sólo representan un problema económico, sino que también son un problema ambiental, de sostenibilidad, operacional y, sobre todo, de salud y seguridad. Desde la óptica económica, evidentemente, la presencia de una cantidad de agua perdida implica un coste asociado a estos volúmenes por el propio recurso en sí mismo y por toda la energía necesaria para la producción, transporte y la potabilización. Un coste ineludible si se quiere hacer llegar agua hasta el punto de consumo. Desde el punto de vista ambiental, los volúmenes fugados implican un exceso de emisión de $\mathrm{CO}_{2}$ a la atmósfera y una sobreexplotación del propio recurso en origen. Evidentemente, un sistema ineficiente y no respetuoso con el medio ambiente nunca conseguirá ser sostenible. Desde la óptica de la operación de los sistemas, las fugas suponen pérdidas de la calidad del servicio. Asimismo, estas fugas pueden desencadenar un colapso del sistema, por rotura de algún elemento, y cortes en el suministro a la población. Por último, y la consecuencia más importante, los escapes de agua atacan directamente a la calidad del agua (Colombo \& Karney, 2002) ya que 
se permite la entrada de patógenos y agentes infecciosos al sistema de distribución durante condiciones de bajas presiones.

El origen, comportamiento y frecuencia de aparición de estos volúmenes incontrolados fugados está ampliamente estudiado (Puust et al., 2010) y aunque son muchos los factores que intervienen (Morris Jr, 1967; AWWA, 1990) se podría destacar la edad y material de las infraestructuras, el nivel medio de presión, la gestión de la red y el estado general de la misma como los más influyentes en su aparición. Una vez iniciado el escape, el volumen fugado se puede producir con mayor o menor magnitud. Atendiendo a este criterio, las fugas se clasifican en tres tipos (Figura 1.2), las cuales tienen que ser localizadas usando métodos distintos:

- Fugas de fondo: Son pequeñas pérdidas reales que no se observan desde la superficie y tienen tan poca magnitud que son indetectables con las tecnologías actuales. No por ello significan un volumen fugado pequeño, todo lo contrario, a largo plazo este componente puede suponer un alto porcentaje dentro del total del volumen perdido, por producirse de manera continuada.

- Fugas no declaradas: Quedan caracterizadas por un caudal de pérdidas mayor que las fugas de fondo, pero no el suficiente como para ser detectadas a simple vista. Por ello, es preciso su búsqueda. El caudal de estas fugas es moderado y su duración depende de la política de gestión y mantenimiento seguida. Es decir, como son detectables con la tecnología actual, se identifican cuando se realiza un plan de búsqueda de roturas.

- Fugas declaradas: Se trata de fallos estructurales de la infraestructura. En este caso, se ocasionan unos caudales de fuga elevados, pero como son rápidamente localizadas y reparadas su duración es pequeña.

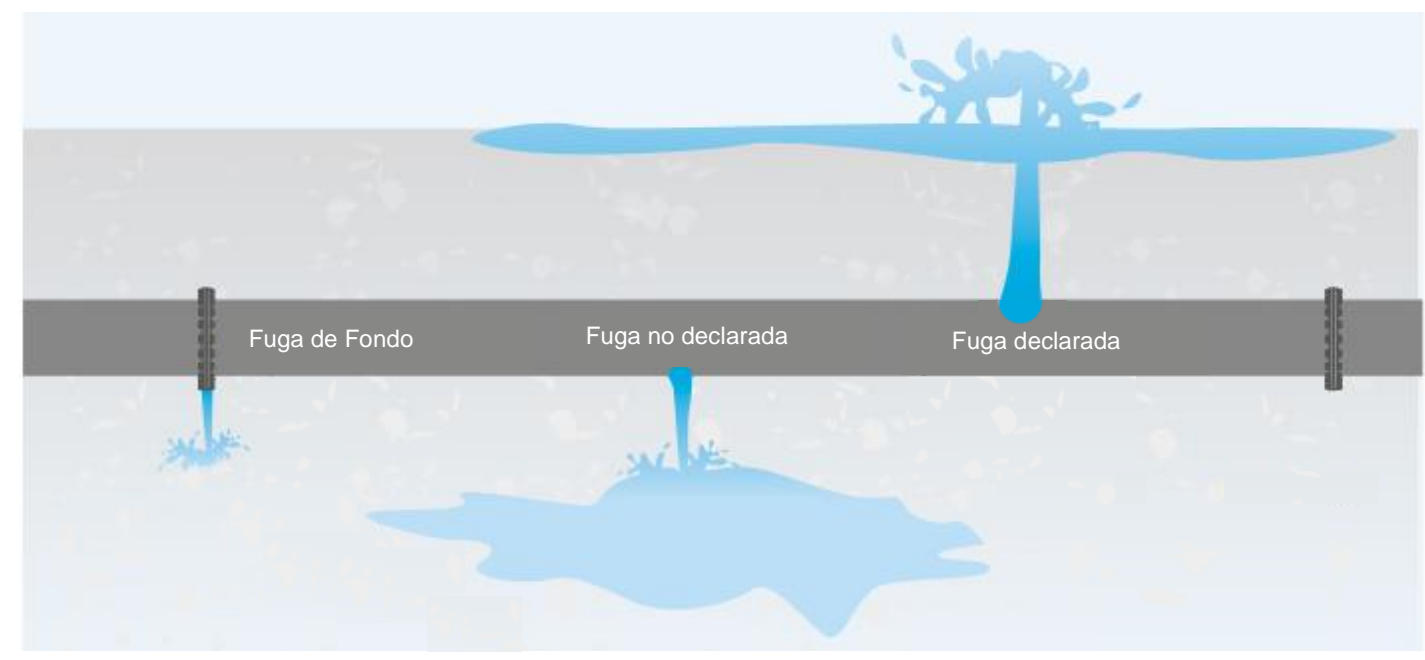

Figura 1.2. Diferentes tipos de fugas según su magnitud (GIZ \& VAG, 2011)

Actualmente, son numerosos los estudios, manuales y libros que abordan el problema de las fugas y sus estrategias de control (GIZ \& VAG, 2011; Farley \& Trow, 2003; Ofwat, 2008; Farley, 2001). De manera agrupada y resumida, el Water Loss Task Force de la International Water Association (IWA) propone cuatro líneas estratégicas de actuación para reducir y controlar este problema. Dichas actuaciones (Figura 1.3) se describen a continuación: 


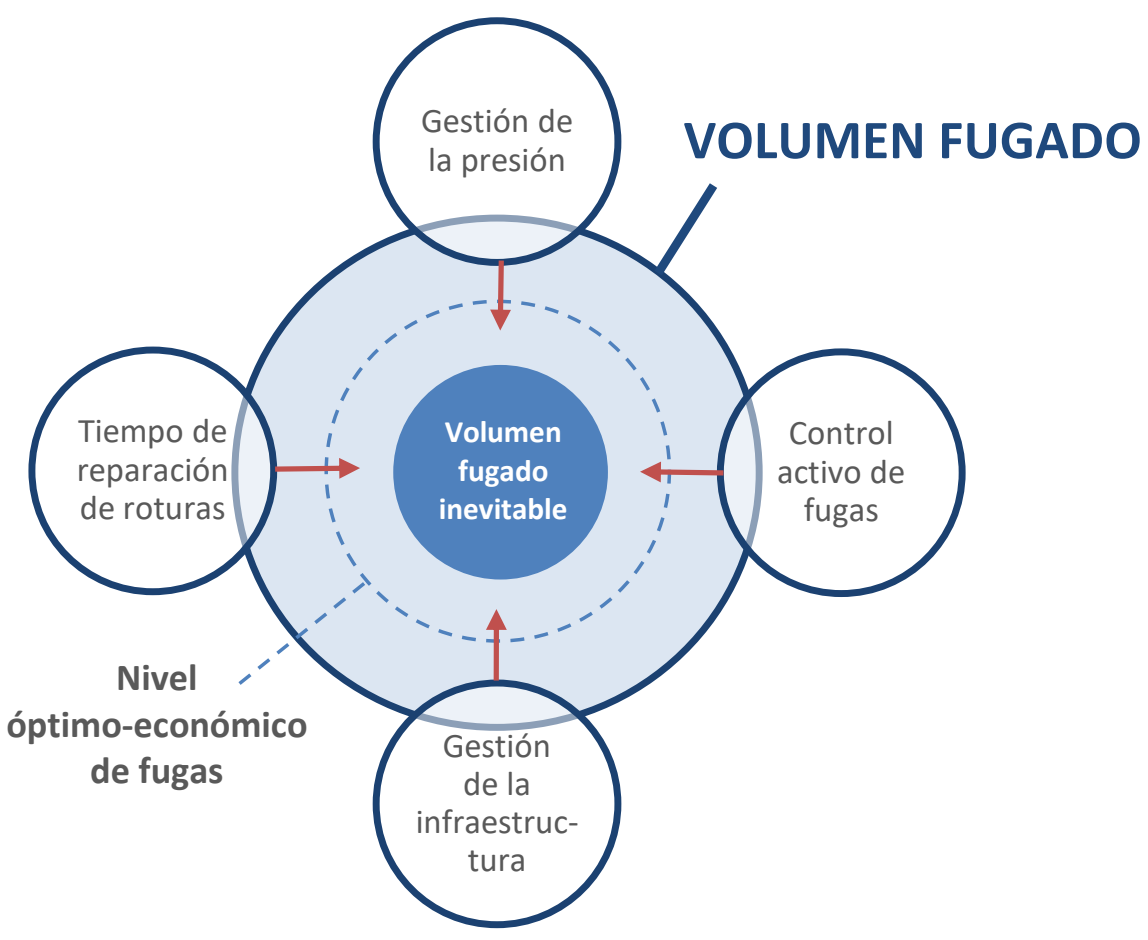

Figura 1.3. Líneas de actuación en la gestión de fugas según el Water Loss Task Force, de la IWA

- Gestión de la presión: Es una actividad clave para reducir pérdidas de agua y disminuir la frecuencia de la aparición de roturas en la red.

Disminuir el nivel de presiones medio influye en el caudal fugado, así como también en el nivel de consumo de la población. El proceso contrario es análogo, a mayor presión, mayores fugas y consumos.

- Tiempo de reparación de roturas: Evidentemente, el mínimo tiempo que se tarde en la reparación desde que es detectada por el usuario o por el equipo de detección y localización de fugas es clave para reducir el volumen fugado.

- Gestión de la infraestructura: Un mantenimiento correctivo y preventivo de todos los componentes del sistema de distribución prevendrá la aparición de pequeños defectos y con ellos, fugas, desde fugas de fondo hasta graves roturas. Poniendo datos a este factor, por ejemplo, el 40\% del parque de tuberías de distribución de agua potable en España, unos $225.000 \mathrm{~km}$, tiene más de 30 años (AEAS \& AGA, 2018). Este lógico deterioro por la edad de todos los elementos favorece la aparición de las fugas.

- Control activo de fugas: Se trata de un conjunto de acciones proactivas ejecutadas para lograr un nivel bajo de pérdidas. La sectorización, las inspecciones del sistema de distribución o las mediciones continuas de flujos nocturnos son algunas de ellas.

Aunque estas cuatro líneas de actuación presentadas son igualmente importantes y su implantación en los sistemas de distribución deber ser conjunta y continua, será la última, el control activo de fugas, sobre la que se centrará este trabajo de investigación.

Puust et al. (2010) realizan un excelente trabajo de recopilación de todas las técnicas empleadas dentro de esta línea de actuación. El estudio abarca métodos o modelos (actuales y pasados) empleados desde antes de conocer la existencia de una fuga hasta el control del nivel fugado. Según la política de activación escogida, se establece un control pasivo de fugas, sobre el cual solo se reparan las fugas declaradas, y un control activo de fugas, en el cual el 
gestor se plantea buscar y reparar todas las fugas, sean declaradas o no (Figura 1.2). Para esta última política de gestión, los autores establecen diferentes grupos de métodos según su objetivo y empleo durante el proceso vital de una fuga.

En primer lugar, establecen los métodos de evaluación de fugas. Su objetivo es cuantificar la cantidad de agua que se está escapando, sin importar dónde se esté produciendo. Según cómo se calcule el volumen fugado, se tienen los métodos de evaluación Top-down o Bottomup. Varios ejemplos de técnicas englobadas en este primer grupo de actuación, globalmente extendidas, son la sectorización de las redes o el método del caudal mínimo nocturno (Liemberger \& Farley, 2005).

Una vez que se cuenta con la certeza de la existencia de la fuga, es necesario pasar a los métodos de localización de ésta. De entre todas las técnicas existentes, destacan los métodos de conocimiento de fuga y de localización. Los primeros se basan en modelos de simulación, no informan de la ubicación exacta y se basan en el análisis del comportamiento del flujo transitorio en grandes tuberías arteriales. Estas técnicas no proporcionan buenos resultados en tuberías con caudales pequeños. El segundo grupo, los métodos de localización, engloba todas las técnicas cuyo objetivo es identificar y priorizar el área donde está la fuga para facilitar su posterior ubicación con precisión. Debido a su uso global, se destacan dentro de este grupo el uso de registradores acústicos (Stephens et al., 2020), el cierre de zonas (step-testing), los sensores de movimiento de tierras y los georradares.

En último lugar, cuando se ha reducido la zona de búsqueda, llegan los métodos de ubicación, que tienen como objetivo el encontrar el punto exacto donde se está produciendo el escape. Las técnicas más extendidas son el uso de correladores, la detección con gas trazador o el uso de hidrófonos.

\subsection{Justificación de la tesis}

El agua ha sido y será un pilar clave en la evolución de la actividad humana. Pero, en la actualidad, son numerosas las amenazas a las que se debe enfrentar, y un uso sostenible de este recurso es la única manera de enfocar dichos problemas.

De entre todas las acciones necesarias para conseguirlo, una que los gestores de los sistemas de distribución de agua deberán abordar es la minimización de los volúmenes incontrolados fugados. Así, se conseguirá ajustar la producción al consumo y con ello un consumo energético ajustado al necesario para dar el servicio, un aumento en la calidad del servicio ofrecido y unos riesgos, principalmente por problemas de salubridad, reducidos.

Por ello, es necesario dotar a los gestores de herramientas, o metodologías, que ayuden a minimizar el problema de los volúmenes incontrolados fugados. Además, estas metodologías deberán presentar un enfoque práctico y sencillo que ayuden en su implantación en la cultura del gestor. Y no solo eso, sino que deberán ser respetuosas, a su vez y de manera global, con todos los parámetros de funcionamiento de los sistemas.

Conocido el problema principal y justificada su solución, se presenta este trabajo de investigación que tiene como título La sectorización basada en criterios energéticos como herramienta para la gestión hídrica de redes de distribución de agua. 


\subsection{Objetivos de la tesis}

La presente tesis pretende profundizar en cómo se realiza la distribución sectorial en las redes de distribución urbana sectorizadas. Esta técnica, la sectorización, es una de las más eficaces en la gestión de los volúmenes incontrolados fugados. Primeramente, se debe comprender el funcionamiento de esta y sus desarrollos, para posteriormente, analizar las posibilidades que permitan una mejor implementación coordinada junto con otras técnicas de gestión. Asimismo, se estudian técnicas alternativas basadas en la sensorización ${ }^{1}$ de las redes buscando un mismo objetivo, la rápida identificación de las fugas, pero sin afectar al comportamiento hidráulico de la red.

Para ello, se ha definido una serie de objetivos generales y específicos que ayudarán a desarrollar y organizar el trabajo de investigación. Estos son:

Objetivo general 1: Proponer una metodología de sectorización semiautomática en la toma de decisiones basada en niveles de energía.

Como primer objetivo de la tesis se propone el desarrollo de una metodología sistemática para el diseño de la sectorización de una red de distribución de agua según criterios energéticos y que facilite la clara delimitación de cada uno de los sectores hidrométricos de la red.

Este objetivo, a su vez, se divide en los tres objetivos específicos, que se describen a continuación:

Objetivo específico 1.1: Aportar nuevos criterios de diseño.

Aunque el proceso de sectorización no ha sido estandarizado, como se detallará en el Capítulo 2, sí que existen una serie de recomendaciones que sirven de guía en una primera delimitación de los sectores hidrométricos: tipo de red, tipo y número de abonados, materiales, trazados, diámetros, avenidas principales, otros tipos de infraestructuras, etc. Muchos de los criterios empleados presentan un carácter heurístico, cuyo desempeño lejos queda de una optimización del funcionamiento hidráulico. Por ese motivo, se propone el uso de criterios energéticos para la definición de los diferentes sectores.

Objetivo específico 1.2: Automatización de diseño de la sectorización.

Uno de los inconvenientes que presenta la técnica de sectorización de redes de distribución de agua recae en su diseño al ser, en la mayoría de los casos, un proceso de prueba y error que depende principalmente de la experiencia del diseñador y cuyo grado de dificultad está vinculado al tamaño y complejidad de la propia red.

Por ello, este segundo objetivo específico plantea la posibilidad de una automatización del diseño de las sectorizaciones. Para ello, emplea un algoritmo semiautónomo de diseño para la definición de los sectores.

\footnotetext{
${ }^{1}$ El término sensorización.... La palabra sensorización es un anglicismo no registrado aún por nuestra RAE, pero en castellano parece difícil encontrar un vocablo que defina mejor que aquella «acción de dotar con múltiples elementos que permitan percibir».
} 
Objetivo específico 1.3: Analizar los beneficios indirectos de la sectorización.

La sectorización de una red implica, en la mayoría de los casos, la modificación de la disposición inicial de la misma al tener que actuar sobre el estado de válvulas de corte para aislar los sectores. Ahora bien, esta alteración influye (positiva o negativamente) sobre la efectividad de otras técnicas de gestión, como por ejemplo la gestión de presiones encaminada a la reducción del volumen incontrolado fugado.

Este tercer objetivo específico plantea analizar la relación entre el diseño de la sectorización y la efectividad u operativa de otras técnicas de gestión, principalmente en aquellas orientadas a la disminución del volumen fugado.

Objetivo general 2: Estudiar y plantear una metodología que permita la búsqueda de fugas sin la necesidad de sectorizar la red.

La sectorización ha resultado ser una técnica eficaz en la búsqueda de fugas, aunque, en contrapartida, como inconvenientes supone una pérdida de capacidad hidráulica y una merma en la calidad del agua. Por ello, se cuestiona el planteamiento de una técnica no intrusiva alternativa que logre los mismos resultados. Por tanto, se propone el desarrollo de una metodología que localice las fugas centrada en la interdependencia de todos los elementos y el empleo de sensores de caudal y presión.

Y este objetivo presenta dos objetivos específicos:

Objetivo específico 2.1: Análisis de sensibilidad de la red.

Se propone un análisis de sensibilidad de la red de distribución medida como la variación del caudal en las líneas y de la presión en los nudos producido por pequeñas variaciones en las demandas. De este modo se identifica el comportamiento medio específico de cada elemento ante un consumo incontrolado ocurrido en cualquier punto de la red, con demanda indeterminada y magnitud variable. Como resultado se obtiene la variación media de los caudales en las líneas y presiones en los nudos ante cualquier incremento de consumo en la red de magnitud dada.

Objetivo específico 2.2: Localización de posición de la fuga.

Teniendo como datos de entrada el conocimiento del comportamiento medio específico de cada elemento ante un incremento de demanda en cualquier punto de la red (obtenido en el objetivo anterior) y la medición obtenida por los sensores instalados, se propone una formulación matricial de las ecuaciones del comportamiento de la red en la que las incógnitas son, directamente, los caudales de fuga en cada nudo, y se resuelven por el algoritmo del gradiente (Todini \& Pilati, 1988).

Objetivo general 3: Comparación de las metodologías.

Como último objetivo general se realizará una comparación entre las dos metodologías planteadas en los objetivos 1 y 2 . Se abordarán también las limitaciones y bondades que presentan cada una de ellas. 


\subsection{Planteamiento del trabajo}

Para dar logro a los objetivos marcados y respuesta a las preguntas planteadas, se plantean dos líneas de trabajo en paralelo: una primera que defienda la necesidad de sectorización y una segunda que promulga lo contrario.

Ambas comienzan con una primera etapa teórica que estudia el comportamiento teórico y físico de todas las metodologías. Para ello, se analizan y estudian nuevos enfoques, basados en la teoría de grafos y en la resolución de algoritmos multiobjetivo, con los que se intentará definir de manera autónoma la sectorización de cualquier red de distribución. Por otro lado, y para defender la no necesidad de sectorizar, se abordan las variaciones de todos los parámetros hidráulicos de la red y se representa dicho comportamiento en un modelo de simulación hidráulica, de modo que la lectura de pocas variables refleje las variaciones en la red.

La investigación teórica se complementa con una segunda fase de aplicación práctica en un modelo. En esta etapa se aplican todas las metodologías planteadas con el fin de analizar los resultados de cada una de ellas y poder así compararlas. Se emplean para ello dos casos de estudio. El primero es una red sintética sencilla de pequeño tamaño, que permite la adecuada comprensión de los desarrollos y de los resultados obtenidos. El segundo es un sistema real de distribución de agua potable.

Para la implantación de las metodologías se cuenta con la ayuda del software Epanet (Rossman, 2002), para la modelación hidráulica de los casos, y su motor de cálculo, a través de su Toolkit, y Microsoft VBA (Visual Basic, a través de Excel), para programar la resolución de los cálculos planteados.

\subsection{Estructura del documento}

La tesis se ha organizado en 6 capítulos (de los cuales, el presente es el Capítulo 1. Introducción y objetivos) y las referencias.

En el Capítulo 2. La técnica de la sectorización se realiza la descripción de esta técnica, se detalla en qué consiste y cómo se ejecuta, se recogen las ventajas e inconvenientes que presenta, así como los principales criterios para su diseño y definición, siempre desde un punto de vista tradicional. Acto seguido, se aborda esta misma técnica bajo nuevos criterios de diseño recogidos en la bibliografía actual.

En el Capítulo 3. Aplicación de conceptos energéticos en la fase de diseño se desarrolla una metodología para la definición de la sectorización de las redes de distribución de agua basada en niveles de energía y de forma autónoma en la toma de decisiones.

Estos dos capítulos, 2 y 3, buscarán la consecución del objetivo general 1 y de los objetivos específicos $1.1,1.2$ y 1.3 .

En el Capítulo 4. Sensores para la localización de fugas se desarrolla una metodología para la localización de los volúmenes incontrolados fugados basada en los datos de presión y caudal a través de la monitorización de las redes y en la sensibilidad hidráulica de las mismas. Con este capítulo se alcanzará el objetivo general 2 y los objetivos específicos 2.1. y 2.2. 
En el Capítulo 5. Casos de estudio se aplican las metodologías desarrolladas en los capítulos 3 y 4 en casos concretos para, posteriormente, realizar una comparación de ambos métodos.

Finalmente, en el Capítulo 6. Conclusiones se establecen las principales conclusiones en base a los resultados y las discusiones alcanzadas.

\subsection{Marco colaborativo: Doctorado Industrial}

Se entiende por Doctorado Industrial la tesis desarrollada en una empresa ajena a la propia Universidad (bien pertenezca al sector privado o Administración Pública). En el Real Decreto 99/2011, de 28 de enero, por el que se regulan las enseñanzas oficiales de doctorado, capítulo 15 se especifica que:

"Se otorgará la mención "Doctorado Industrial" siempre que concurran las siguientes circunstancias:

a) La existencia de un contrato laboral o mercantil con el doctorando. El contrato se podrá celebrar por una empresa del sector privado o del sector público, así como por una Administración Pública.

b) El doctorando deberá participar en un proyecto de investigación industrial o de desarrollo experimental que se desarrolle en la empresa o Administración Pública en la que se preste el servicio, que no podrá ser una Universidad. El proyecto de investigación industrial o de desarrollo experimental en el que participe el doctorando debe tener relación directa con la tesis que realiza. Esta relación directa se acreditará mediante una memoria que tendrá que ser visada por la Universidad.

2. En el caso de que el proyecto de investigación industrial o de desarrollo experimental se ejecute en colaboración entre la Universidad y la empresa o Administración Pública en la que preste servicio el doctorando, se suscribirá un convenio de colaboración marco con entre las partes. En este convenio se indicarán las obligaciones de la Universidad y las obligaciones de la empresa o Administración Pública, así como el procedimiento de selección de los doctorandos."

En el Real Decreto 195/2016 se introducen las menciones de doctorado internacional y de doctorado industrial por razones de interés general. En este Real Decreto se especifica que "Como quiera que estas dos menciones son consecuencia de la expresa regulación de estos dos tipos de Doctorado en el Real Decreto 99/2011, de 28 de enero, se hace necesario contemplar dicha denominación en el Real Decreto 1002/2010, de 5 de agosto, precisamente, para que sea posible llevar a cabo la expedición del correspondiente título de Doctor" y además añade "En la actualidad hay Universidades que pueden introducir en sus estudios de Doctorado el concepto de Doctorado industrial. Si el Real Decreto de Doctorado 99/2011, de 28 de enero, no contempla de forma expresa este tipo de Doctorado lo único que hace el Estado es perjudicar a los estudiantes que pueden cursar este tipo de estudios universitarios, comoquiera que no se les reconoce en el título, la mención de Doctor industrial. Este tipo de Doctorados se realizarían con la colaboración del tejido empresarial para garantizar los lazos entre el mundo académico y el mundo empresarial, ya sea éste empresa pública o empresa privada.". 
De este modo, el doctorado industrial pretende fomentar la investigación en el tejido empresarial y la participación de la industria en los programas de doctorado, incentivar la transferencia de conocimiento entre la universidad y su entorno, e impulsar la inserción laboral de los jóvenes investigadores. Todo esto en cualquier área de conocimiento, desarrollando un proyecto investigación industrial o de desarrollo experimental.

La presente tesis ha sido desarrollada en el seno de una empresa del sector privado y aborda uno de los principales problemas de los gestores de los sistemas de distribución de agua. Se opta a la mención de Doctorado Industrial por la Universitat Politècnica de València ya que se cumplen las condiciones de contrato laboral entre doctorando y empresa (Aguas de Valencia S.A.) y que el producto de la investigación ha consistido en la implementación directa del mismo en una red de abastecimiento de agua potable. La segunda condición se cumple con el convenio firmado entre la Universitat Politècnica de València y Aguas de Valencia y la aprobación del proyecto denominado Sensorización de la red como herramienta para la detección de la ubicación de volúmenes dentro de la línea de ayudas en materia de fortalecimiento y desarrollo del Sistema Valenciano de Innovación (Agència Valenciana de la Innovació $(\mathrm{AVI})$ ), concretamente en la línea de actuación de Promoción de Talento de Doctorandos empresariales (Innodocto).

Por tanto, se ha conseguido así un excelente marco de colaboración entre la Universitat Politècnica de València y la empresa privada que, además de la presente tesis doctoral, ha contribuido a enriquecer en diferentes ámbitos. En la empresa privada, se han obtenido metodologías entendibles y replicables para la gestión de los volúmenes incontrolados. La sencillez de las herramientas son un elemento diferenciador para su posterior implantación entre los gestores de redes de abastecimiento. Se consigue así, fomentar la investigación en el tejido empresarial y la participación de la industria en los programas de doctorados, incentivar la transferencia de conocimiento entre la universidad y su entorno, e impulsar la inserción laboral de los jóvenes investigadores. Los objetivos de esta tesis coinciden plenamente con la línea estratégica de Aguas de Valencia S.A., ya que la reducción del volumen incontrolado fugado es uno de los pilares dentro su política de gestión eficiente (Global Omnium, 2019).

Para finalizar, la divulgación de los principales trabajos y conclusiones obtenidos en las diferentes líneas investigadas ha contribuido a enriquecer, en el ámbito académico, a la comunidad científica. Así, se pueden destacar las siguientes contribuciones que esta tesis ha aportado durante su periodo de desarrollo:

- Salguero, F. J., Cobacho, R., \& Pardo, M. A. (2019). New approaches in the current management of water losses in distribution networks. 10th IWA Specialist Conference on Efficient Use and Management of Water. Manila, Philippines

- Salguero, F. J., Cobacho, R., \& Pardo, M. A. (2019). Sensorización de las redes de distribución de agua como herramienta en la localización de fugas. VI Jornadas de Ingeniería del Agua (JIA 2019). Toledo, España.

- Salguero, F. J., Cobacho, R., \& Pardo, M. A. (2018). Segmentation vs Pressure Sensing: A decision support system to obtain the best alternative for water leakage detection in water distribution networks. 5th Water Efficiency Conference (WATEFCon 2018). Future of Water in Europe: Local, regional, and global best practice. Aveiro (Portugal). 
- Salguero, F. J., Cobacho, R., \& Pardo, M. A. (2018). Unreported leaks location using pressure and flow sensitivity in water distribution networks. Water Science and Technology: Water Supply, ws2018048. doi:10.2166/ws.2018.048.

- Salguero, F. J., Cobacho, R., \& Pardo, M. A. (2017). Water losses identification using pressure and flow sensitivity in water distribution networks. 9th IWA Specialist Conference on Efficient Use and Management of Water. Bath (United Kingdom)

- Salguero, F. J., Cobacho, R., \& Pardo, M. A. (2017). Sectorización de redes de distribución de agua según criterios de eficiencia energética. V Jornadas de Ingeniería del Agua (JIA 2017). La Coruña, España. 


\section{Capítulo 2 La técnica de la sectorización}

\subsection{Introducción}

La vida de una fuga es el tiempo que transcurre desde que se origina el escape de agua hasta que se repara el defecto que lo provoca, y se puede calcular como la suma de los tiempos transcurridos durante las fases de evaluación, detección y localización y ubicación. La primera fase engloba el periodo desde que se produce el escape hasta que se conoce de su existencia, y su duración dependerá, en gran medida, de las infraestructuras existentes, el tamaño de la red, los sistemas de control y la política de gestión de fugas seguida. Tras conocerse la existencia de fugas, es necesario la detección y localización en la zona donde se estén produciendo. Por ello, la duración de esta fase dependerá del tamaño y su búsqueda. La duración de la última fase, los trabajos de ubicación, está vinculada en exclusiva a la experiencia de los técnicos encargados de ello, y los métodos y tecnologías empleadas.

Para reducir la vida de las fugas, los gestores no han parado de trabajar durante el último siglo (Pilcher, 2003) con el objetivo de acortar la duración de cualquiera de las fases anteriores. Y han sido varias las metodologías empleadas con el mismo fin. Véase para ello la red de distribución mostrada en la Figura 2.1. Se trata de una red de distribución de agua por gravedad desde un único depósito de cabecera, y que cuenta con un caudalímetro en el punto de entrada al sistema. Si se quiere conocer la existencia de fugas o cuantificar el volumen fugado, se puede seguir alguno de los siguientes tres métodos. En primer lugar, se puede recurrir a la práctica conocida como Step-Testing (Boulos \& Aboujaoude, 2011). Esta técnica consiste en aislar pequeñas zonas durante las horas de menor consumo. Durante estos periodos, teóricamente, no se están produciendo consumos o estos son mínimos, siendo la mayor parte del volumen inyectado al sistema volumen incontrolado fugado. Si durante las operaciones de cierre, no se produce disminución en el caudal inyectado, esto significará que en la zona desconectada no existen fugas. En cambio, si al aislar una zona se produce un descenso en el caudal inyectado, se puede atribuir este descenso al volumen incontrolado 
fugado. Con este método se puede conseguir acotar la localización a una zona concreta, pero se trata de un método manual, que provoca cortes en el servicio, cuya efectividad dependerá del número, estado y ubicación de las válvulas de corte instaladas en la red y con el que, al hacer cerradas durante periodos muy cortos de tiempo, no se puede descartar consumo ante una bajada del caudal inyectado.

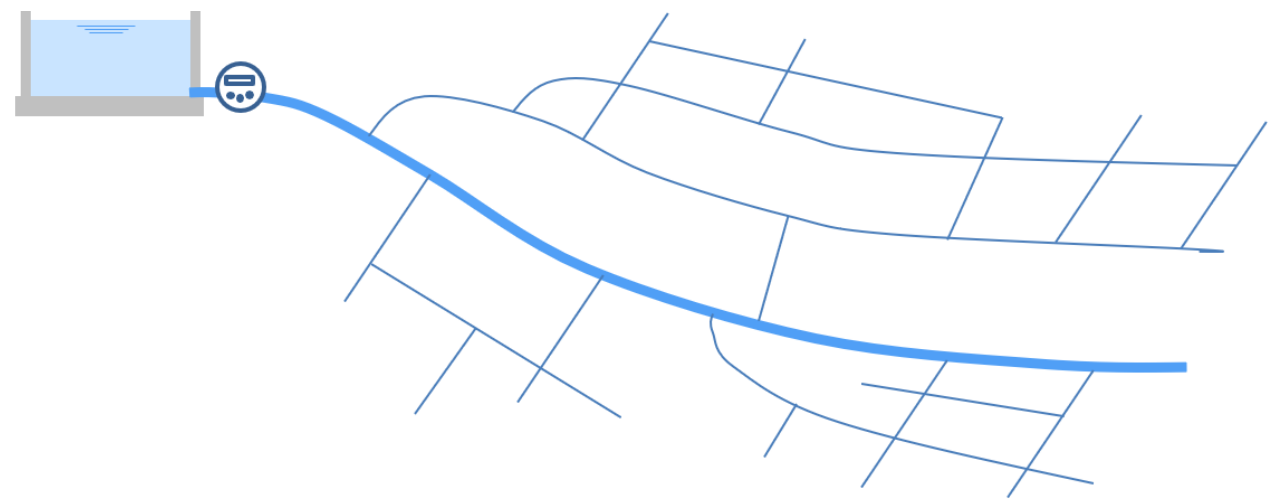

Figura 2.1. Red de distribución de agua con un contador en el punto de entrada

Un segundo método, para conocer cuánto volumen se está escapando del sistema, es el del caudal mínimo nocturno (Liemberger \& Farley, 2005). Este método se basa en la hipótesis de que un gran porcentaje del volumen inyectado a un sistema durante las horas nocturnas es debido a las fugas, ya que no se estará produciendo consumo durante estas horas, o este será mínimo. A diferencia del Step-Testing, en el método del caudal mínimo nocturno se realiza un seguimiento más completo del consumo, lo que permite conocer el comportamiento del flujo inyectado y comprobar si existe o no consumo, así como obtener una estimación del nivel fugado en el sistema. En todo caso, la efectividad de este método dependerá del tamaño de la infraestructura, así como de la naturaleza y comportamiento de los consumos. El último método para obtener el volumen fugado, y más generalmente establecido entre los gestores, consiste en la realización del balance hídrico del sistema. Así, se obtiene el volumen incontrolado fugado medio de todo el sistema durante el periodo estudiado. La realización de este análisis es sencilla, pero requiere de todos los datos de consumo para ello.

Los anteriores métodos pueden ser usados de manera individual, o de manera conjunta para potenciar los resultados obtenidos. E incluso, pueden mejorarse para reducir aún más el tiempo medio de la vida de las fugas. Persiguiendo este objetivo, en los años 80 vio la luz uno de los estudios pioneros referente a este tema: la Water Authorities Associations publicó el informe n²6, realizado por el Technical Working Group on Waste of Water, el cual tiene como título Leakage Control Policy and Practice (Water Authorities Association, 1985). En dicho informe, el grupo de trabajo plasmó los métodos más apropiados en el control de los volúmenes incontrolados fugados que estaban empleando, en aquel momento, las empresas especializadas de Reino Unido. Y entre todos los presentados habría que destacar una metodología seguida en las ciudades de Plymouth y Rickmansworth (Farley, 2010). En dichos casos, y vistas las ventajas e inconvenientes que presentaban las anteriores técnicas, dividían la red de distribución en varias subredes más pequeñas e independientes entre sí. Así, conseguían sistemas más pequeños que facilitaban un mejor control de las anomalías y una mayor rapidez en las dos primeras fases de la búsqueda de fugas. Esta técnica quedó bautizada como sectorización, y en la actualidad es una de las técnicas más empleadas por los gestores por ser una de las mejores contribuciones al ratio coste/efectividad y eficiencia dentro de la gestión de fugas (Di Nardo \& Di Natale, 2010; Farley \& Liemberger, 2005; Sarbu, 
2009; Sturm \& Thornton, 2005). Años después se ha podido comprobar que la sectorización de las redes no solo ha contribuido a la gestión de las fugas, sino que la gestión de la calidad del agua (Özdemir, 2018) o la gestión de la presión (Galdiero et al., 2016; Saldarriaga, 2016) se ven también mejoradas. De hecho, la partición de una red de agua, una metodología similar a la sectorización divide el sistema en distritos independientes, cada uno de ellos alimentado por su fuente de agua sin conexiones a otros distritos, con el fin de lograr zonas con suministro independiente (Tzatchkov et al., 2006).

\subsection{Ejecución de la técnica}

Las redes urbanas de distribución de agua presentan un diseño mallado. Esto es así debido a que, durante muchos años, el diseño de estos sistemas ha buscado la resiliencia y la capacidad del suministro. Si bien, un sistema mallado presenta muchas ventajas, no facilita los trabajos centrados en el control activo de fugas. Si se coge, por ejemplo, un sistema de estas características con un único punto controlado de suministro solo será posible conocer cuánto volumen se está escapando del sistema sin ser registrado de manera global. Así, tampoco es posible conocer dónde se está escapando. Ahora bien, es posible ir cerrando tuberías y creando, así, zonas aisladas dentro del total del sistema mallado. En el momento en que se equipe la tubería de suministro con un medidor de caudal se estará creando un Distric Metered Area (DMA), conocido como sector. Y la técnica en su conjunto se le denomina sectorización.

Según se ha apuntado en el apartado anterior, sectorizar una red se basa en fraccionarla en varias subredes, todas ellas controladas. Reside, en estas divisiones, la eficacia de esta técnica, dado que se ha producido una división en zonas de menor tamaño. La eficacia en la detección de anomalías aumenta al ser más sencilla la interpretación del comportamiento del caudal inyectado. Además, con esta distribución de zonas independientes es posible la definición de la intensidad y la distribución espacial y temporal de las fugas.

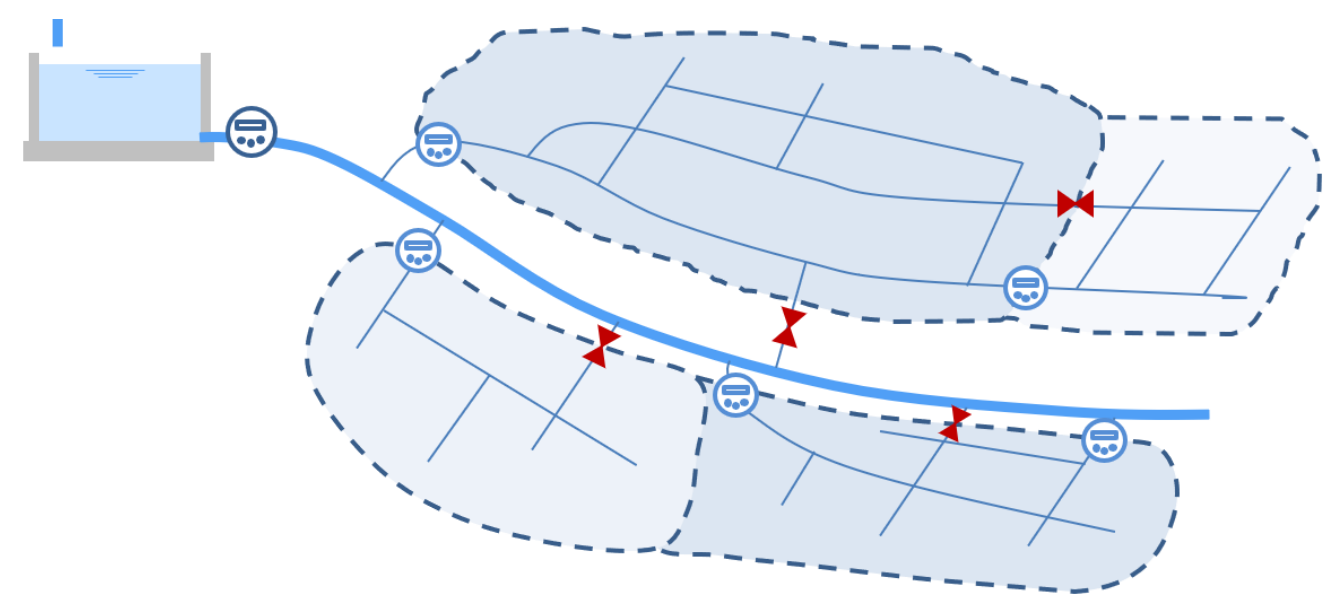

Figura 2.2. Red de distribución de agua sectorizada

En cambio, si se quiere actuar de manera táctica sobre el problema (Alegre \& Coelho, 2012) será necesario aislar diferentes zonas de la red de distribución para poder estudiarlas por separado. 
Una vez creados los sectores, el siguiente paso será el análisis de los caudales circulantes por las tuberías de suministro. Se conseguirá, así, una agilización en la gestión de las infraestructuras, principalmente en lo referente al control activo de fugas y la gestión de la presión, pero que conlleva limitaciones desde el punto de vista del diseño, la calidad del agua distribuida y la robustez del propio sistema de distribución (Tzatchkov et al., 2014).

Desde el punto de vista de la planificación, esta técnica se estructura en las siguientes fases:

- Fase I - Diseño de la sectorización: La primera etapa consiste en la definición de los propios sectores, en la delimitación de las fronteras de estos. Es, sin duda, la base del diseño (Diao et al., 2013) y la fase más complicada y que marcará el grado del éxito conseguido. En esta fase, y de cara a una ejecución exitosa, es aconsejable el uso de un modelo de simulación hidráulico que permita analizar y evaluar diversos escenarios de funcionamiento de la red. En caso de no disponer del mismo, diseñar una sectorización para un gran sistema se basará en un proceso de prueba y error sin ningún fundamento técnico.

- Fase II - Ejecución de la sectorización: Una vez se conoce cómo debe ser la sectorización, esto es, dónde deben colocarse los contadores sectoriales y qué tuberías deben cerrase, el siguiente paso consiste en la ejecución de dicha sectorización. Se instalan los contadores y las válvulas de seccionamiento necesarias.

- Fase III - Operación y Control: Tras las fases de proyecto y ejecución, que tienen una duración relativamente corta, llega la última etapa de la sectorización que consiste en su operación. Se entiende por operación el conjunto de todos los trabajos de análisis de los consumos para la detección de anomalías, tales como fugas o roturas. Estos trabajos de operación quedan englobados dentro de la gestión de las infraestructuras, más concretamente, en la línea de trabajos de control activo de fugas.

Una de las debilidades que presenta el método de Step-Testing reside en que los cierres temporales aíslan totalmente la zona, dejándola sin suministro. Para mejorar dicha deficiencia, con la sectorización se consigue aislar zonas, de manera permanente, sin desatender suministro alguno. Este hecho, que ayuda en el control activo de las fugas, puede penalizar la fiabilidad y robustez del sistema. Por ello, autores como Wright et al. (2015) o Di Nardo et al. (2016) proponen el empleo de válvulas de control remoto que permitan modificar las fronteras de los sectores según el punto de funcionamiento requerido (roturas en la red, incendios, reducción de los niveles medios de presión, etc.). Esta modalidad se conoce como sectorización dinámica.

\subsection{Ventajas e inconvenientes}

La sectorización de las redes nació con el principal objetivo de hacer frente al problema de los volúmenes fugados y mejorar, con ello, la propia gestión de las infraestructuras. Pero esta no es la única ventaja que con ella se consigue, tal y como citan numerosos de autores. A continuación, se recogen las principales ventajas que se consigue con la sectorización de las redes de distribución de agua:

- Incremento en la capacidad de detección de fugas o anomalías: La principal ventaja que presenta esta técnica consiste en el aumento de la capacidad de detección de fugas o anomalías en la red. Esto es debido a la división de la red y al mayor control 
de esta. Y es ahí donde reside la eficacia de esta técnica. A diferencia de la Figura 2.1, la disposición de la red de la Figura 2.2 cuenta con mayor número contadores, los cuales registran unos caudales menores que los trasegados por el contador general. Esto permite detectar, sobre cada uno de los contadores sectoriales, desviaciones de consumo indetectables en el contador general.

- Reducción del área de búsqueda de fugas: En línea con la anterior ventaja, el simple hecho de dividir la red en varias subredes independientes y controladas permite conocer cuándo se produce una anomalía y en qué sector se está produciendo. Gracias a ello, la carga de trabajo necesaria para la ubicación de la fuga será menor y, por ende, el tiempo de búsqueda.

- Limitación del área de influencia de anomalías o contaminantes: En caso de producirse algún tipo de anomalía o contaminación en la red, una red sectorizada reducirá sus efectos negativos tan solo a la extensión del sector afectado gracias a las desconexiones entre las diferentes zonas (Grayman et al., 2009; Tzatchkov et al., 2014). Tanto es así que Grayman et al. (2016) definen la sectorización como un diseño pasivo contra la contaminación, pudiendo llegar a reducir el número de personas y kilómetros afectados en un 60\% y 89\%, respectivamente (Di Nardo et al., 2014).

- Mejora en la evaluación de los trabajos dentro del control activo de fugas: Una mayor definición de los consumos en la red permitirá evaluar con mayor claridad los resultados de las campañas de reparación de fugas o gestión de presiones en la red, por ejemplo.

- Gestión de la presión: La gestión de la presión puede ser una tarea complicada sobre una red mallada debido a la gran cantidad de condiciones de contorno existentes. Por ello, muchos autores (Awad et al., 2009) aprovechan la simplificación de la red que les brinda la sectorización para implantar una gestión de presión más efectiva y fácil de proyectar. Se puede ejecutar una gestión de presiones dentro de cada sector, siguiendo un diseño tradicional de optimización (Araujo et al., 2006), o se puede equipar el punto de entrada al sector con una válvula reductora de presión para reducir los niveles excesivos de presión durante momentos puntuales a lo largo del día (Gomes et al., 2013).

- Gestión de la red: En último lugar, la sectorización permitirá aplicar un enfoque táctico a los trabajos de gestión de la red (Alegre \& Coelho, 2012), permitiendo la priorización de las diferentes zonas dentro de una red de futuros trabajos o intervenciones.

Como se ha visto, esta técnica presenta numerosas ventajas, pero en contra, y por el simple hecho de operar de manera directa sobre los elementos de la red, la sectorización lleva asociada una serie de inconvenientes:

- Pérdida de fiabilidad y robustez del sistema: Una de las tareas a realizar en la sectorización es el cierre de tuberías para aislar sectores. De esta manera, se está ramificando la red con la consiguiente disminución de garantía de suministro respecto a la red mallada de partida. Desde el punto de vista de la fiabilidad, la red está perdiendo posibles caminos de transporte, con lo que la probabilidad de fallo en el tiempo aumenta (Yazdani \& Jeffrey, 2012). En cuanto a la robustez, por el mismo motivo, la red perderá capacidad hidráulica para realizar el trasporte del caudal requerido, pudiendo producirse una disminución de la presión y creando puntos críticos 
(Farley \& Liemberger, 2005). Siguiendo esta línea, Campbell (2017) destaca la posibilidad de aumentos de consumo energético en algunos sistemas para hacer frente a estos cierres de tubería. Por otra parte, cabe destacar que, esta pérdida de fiabilidad puede ser muy pequeña o despreciable (Grayman et al., 2016).

- Aumento del tiempo de permanencia: Al realizar cierres de tuberías, se está aumentando el recorrido del agua hasta el punto de consumo e incrementando el número de zonas finales de trayecto, puntos muertos, donde esta queda estancada (Farley \& Liemberger, 2005). Estos motivos hacen que el agua permanezca más tiempo dentro de las redes mermando los estándares de calidad (Armand et al., 2015; Grayman et al., 2009). Aunque este aumento del tiempo de permanencia del agua en la red dependerá de cada sistema, pudiendo ser un valor despreciable (Grayman et al., 2016), por norma general lleva asociado una serie de problemas que dependerán del mismo (Tabla 2.1).

Tabla 2.1 Resumen de los problemas de calidad del agua asociados con el tiempo de permanencia de esta en la red (EPA, 2002)

\begin{tabular}{|c|c|c|}
\hline Problemas químicos & Problemas biológicos & Problemas físicos \\
\hline $\begin{array}{c}\text { Formación de subproductos de } \\
\text { desinfección }\end{array}$ & $\begin{array}{c}\text { Biodegradación de subproductos } \\
\text { de desinfección }\end{array}$ & Aumento de la temperatura \\
\hline Descomposición del desinfectante & Nitrificación & Deposición de sedimentos \\
\hline Eficacia del control de corrosión & $\begin{array}{c}\text { Recrecimiento, recuperación y } \\
\text { blindaje microbiano }\end{array}$ \\
\hline Sabor y olor & Salor y olor & \\
\hline
\end{tabular}

- Inversión económica: En último lugar, implantar la técnica de la sectorización lleva aparejado, necesariamente, un gasto. Inicialmente, será necesario equipar la infraestructura según los requisitos estudiados en la fase de diseño. Tras ello, la sectorización de la red requiere de unos costes de mantenimiento anuales para el correcto funcionamiento de los equipos y las comunicaciones. $Y$, en algunos casos, puede llegar a ser necesario reforzar ciertos tramos de tubería para garantizar la presión de suministro.

Una vez detalladas las principales ventajas e inconvenientes, a modo resumen se muestran, en la Tabla 2.2, todas ellas:

Tabla 2.2 Ventajas e inconvenientes de la técnica de la sectorización

\begin{tabular}{|c|c|}
\hline Ventajas & Inconvenientes \\
\hline $\begin{array}{c}\text { Incremento en la capacidad de detección de fugas o } \\
\text { anomalías }\end{array}$ & Ramificación de las redes \\
\hline $\begin{array}{c}\text { Disminución del área de búsqueda de cada anomalía } \\
\text { particular }\end{array}$ & Disminución de la fiabilidad del suministro \\
\hline $\begin{array}{c}\text { Disminución del área de influencia de las anomalías y } \\
\text { aumento de la capacidad para aislar zonas en } \\
\text { respuestas a estas }\end{array}$ & Disminución de la robustez del sistema \\
\hline
\end{tabular}




\begin{tabular}{|c|c|}
\hline Ventajas & Inconvenientes \\
\hline Evaluación de los trabajos de control activo de fugas & $\begin{array}{c}\text { Aumento del tiempo de permanencia del agua y } \\
\text { creación de puntos muertos (disminución de la } \\
\text { calidad del agua) }\end{array}$ \\
\hline $\begin{array}{c}\text { Priorización de zonas y determinación de los } \\
\text { momentos de intervención de búsqueda de fugas }\end{array}$ & $\begin{array}{c}\text { Necesidad de una inversión económica inicial y de } \\
\text { operación }\end{array}$ \\
\hline $\begin{array}{c}\text { Posibilidad de reducción de la presión y, con ello, del } \\
\text { número de roturas }\end{array}$ & $\begin{array}{c}\text { Disminución de la presión y creación de nuevos } \\
\text { puntos críticos }\end{array}$ \\
\hline Ayuda en la gestión de presiones & Posibles aumentos del consumo energético \\
\hline
\end{tabular}

Como se ha desarrollado en este apartado, la sectorización de una red de distribución presenta muchas ventajas y, también, inconvenientes sobre los objetivos principales de esta infraestructura. $Y$ aunque el ahorro de agua producido debería prevalecer frente a los pequeños inconvenientes ocasionados, es necesario realizar un análisis comparativo entre los beneficios y costes (Ferrari \& Savic, 2015) o entre los aspectos positivos y negativos de la técnica (Campbell et al., 2016) para poder cuantificar, de manera global, la efectividad de esta técnica en cada sistema. En esta línea, Savić \& Ferrari (2014) concluyen que tras la sectorización de una red se puede observar una disminución, en función del número de sectores, en la robustez del sistema y el tiempo de permanencia del agua, pero que son irrelevantes comparados con los beneficios obtenidos en términos de reducción de fugas, mejora en la seguridad y un mejor control y gestión de la red.

\subsection{Diseño tradicional}

Debido a la gran cantidad de criterios que intervienen (tipo de red, tipo y número de abonados, materiales, trazados, diámetros, avenidas principales, otros tipos de infraestructuras, etc.), la mayoría de los autores coinciden en la complejidad a la hora de realizar el diseño de la sectorización (Alvisi \& Franchini, 2014).

Tradicionalmente y de manera generalizada, el diseño de esta técnica ha estado supeditado a la experiencia del diseñador con procesos de prueba y error (Di Nardo et al., 2013; Farley, 2008; Grayman et al., 2016; Tzatchkov et al., 2014), y cuyo punto de partida eran elementos que facilitaran la delimitación entre sectores, como grandes avenidas, barrios, otras infraestructuras o cualquier otro tipo de delimitaciones naturales. Una vez predefinidos algunos sectores, el resto se conseguían con el cierre de tuberías y comprobando su efecto sobre la presión de los nudos dependientes, terminando el proceso cuando el diseñador así lo consideraba. En el caso de tener un modelo de simulación, las pruebas se podrían realizar sobre el mismo hasta conseguir un escenario sectorizado que cumpliera con los requisitos impuestos.

Respecto a los criterios seguidos por los diseñadores, no se han dictado unos estándares fijos para la definición de cada sectorización o cada sector. Por el contrario, sí existen varias recomendaciones para ello (Tabla 2.3), siendo los principales factores los siguientes (Morrison et al., 2007): 
- Orografía: La definición de sectores según niveles de cotas del terreno, buscando con ello niveles de presiones homogéneos, es uno de los principales criterios seguidos por la mayoría de los diseñadores (GIZ \& VAG, 2011).

- Tamaño: No se recomiendan sectores con una gran extensión (Farley M. , 2001). Eso significaría mayores demandas, mayores tiempos de búsqueda de fuga, menor eficiencia en la gestión de la presión y caudales de entrada al sector mayores.

- Patrones de consumo similares: Si el patrón de comportamiento de consumo en todos los abonados es homogéneo, con mayor facilidad se pueden estudiar y detectar anomalías debido a variaciones en los caudales de entrada. Por ello, se recomienda separar en sectores diferentes los consumos diferentes.

- Número de tuberías de suministro: Uno de los requisitos que debe cumplir una sectorización es poder medir el agua de manera práctica y económica (Tzatchkov et al., 2014). En esta línea, son muchos los autores que defienden la única entrada por sector (Di Nardo et al., 2013), o el menor número de estas.

- Otras infraestructuras urbanas: En muchos casos, son los elementos urbanos existentes, tales como grandes avenidas o líneas de tren, los que marcan el punto de partida en la delimitación de los sectores.

- Volumen incontrolado fugado: Algunos autores determinan el tamaño de los sectores fijando como criterio cuánto volumen incontrolado fugado esperan englobar dentro cada uno de ellos (Farley M. , 2001). En el caso de fijar este valor, el tamaño del sector dependerá del estado de la infraestructura y la presión media. En cambio, si se quiere establecer un nivel económico de fugas, el tamaño dependerá, además, del coste de intervención (Hunaidi, 2005).

- Calidad del agua: Este factor es más una restricción que un criterio. No se han encontrado recomendaciones de calidad del agua de cara al diseño, en cambio, habitualmente se comprueba que el tiempo de permanencia (Rossman, 2002) de la red sectorizada no sobrepase unos valores establecidos como límites por el diseñador. Pero en caso contrario, esto no será un factor determinante de la no ejecución de un sector, sino la justificación de la necesidad de tomar medidas complementarias, como campañas de purga o ampliación de la red (Sturm \& Thornton, 2005; Tzatchkov et al., 2014).

- Presiones mínimas de servicio y capacidad frente a incendios: Al igual que el anterior, otros requisitos que debe cumplir la sectorización de toda red es una presión mínima en cualquier punto, mayor que la presión mínima de servicio, y una capacidad hidráulica suficiente para poder cumplir en el punto más desfavorable con las condiciones legales de caudal y presión ante situaciones de incendio.

No obstante, existen otras recomendaciones menos presentes, pero no por ello menos importantes: MacDonald \& Yates (2005) indican que el tamaño máximo para cada sector, siguiendo las indicaciones de la Halifax Regional Water Commission, debe permitir, una vez detectada una fuga, el poder inspeccionarlo en un solo día. Siendo así, se deberá englobar un máximo de 150-200 hidrantes, 2500 acometidas o 30 kilómetros de tuberías. Respecto a las tuberías de suministro a los sectores, se recomienda seleccionar el menor diámetro posible, para detectar el flujo nocturno con mayor sensibilidad, pero que sea capaz de cumplir 
con los requisitos contraincendios. Respecto al tamaño de los sectores, Ferrari et al. (2014), siguiendo las indicaciones de Farley (1985) y Morrison et al. (2007), establecen un rango mínimo y máximo de acometidas por sector, entre 500 y 5000. De Paola et al. (2014) recomiendan un rango mínimo y máximo de demanda que deben englobar los sectores, según el número de acometidas.

A modo de resumen, la Tabla 2.3 contiene las principales recomendaciones recogidas en la bibliografía.

Tabla 2.3 Principales criterios para el diseño de las sectorizaciones

\begin{tabular}{|c|c|c|c|c|}
\hline Criterio & Subcriterio & Recomendación & Autor & Comentarios \\
\hline \multirow{12}{*}{$\begin{array}{l}\text { TAMAÑO DEL } \\
\text { SECTOR }\end{array}$} & \multirow{7}{*}{$\begin{array}{l}\text { Número de } \\
\text { acometidas }\end{array}$} & 2.000 & $\begin{array}{l}\text { (Charalambous et al., } \\
\text { 2014) }\end{array}$ & \\
\hline & & 2.500 & (MacDonald \& Yates, 2005) & \\
\hline & & $500-5.000$ & $\begin{array}{c}\text { (Ferrari et al., 2014) } \\
\text { (Farley, 1985) } \\
\text { (Morrison et al., 2007) } \\
\text { (Grayman et al., 2016) }\end{array}$ & \\
\hline & & $1.000-2.500$ & (Farley, 2008) & \\
\hline & & $500-3.000$ & $\begin{array}{c}\text { (EPA, 2010) } \\
\text { (Gomes et al., 2013) } \\
\text { (Morrison et al., 2007) }\end{array}$ & \\
\hline & & $<500$ & (Morrison et al., 2007) & $\begin{array}{c}\text { Si el estado de la } \\
\text { infraestructura es } \\
\text { malo }\end{array}$ \\
\hline & & $<1000$ & (Farley M. , 2001) & $\begin{array}{l}\text { Sectores } \\
\text { pequeños }\end{array}$ \\
\hline & $\begin{array}{l}\text { Número de } \\
\text { hidrantes }\end{array}$ & $150-200$ & (MacDonald \& Yates, 2005) & \\
\hline & \multirow{3}{*}{$\begin{array}{l}\text { Longitud de } \\
\text { red }\end{array}$} & $30 \mathrm{~km}$ & (MacDonald \& Yates, 2005) & \\
\hline & & $4-30 \mathrm{~km}$ & (GIZ \& VAG, 2011) & \\
\hline & & Longitud óptima & (Hunaidi, 2005) & $\begin{array}{c}\text { Cálculo según los } \\
\text { costes de } \\
\text { intervención }\end{array}$ \\
\hline & $\begin{array}{l}\text { Tiempo de } \\
\text { inspección }\end{array}$ & 1 día & (MacDonald \& Yates, 2005) & \\
\hline \multirow{5}{*}{$\begin{array}{c}\text { TUBERÍAS DE } \\
\text { ALIMENTACIÓN } \\
\text { Y No DE } \\
\text { CONEXIONES }\end{array}$} & \multirow{5}{*}{ Conexiones } & $\begin{array}{l}\text { Ninguna conexión } \\
\text { entre sectores }\end{array}$ & (Ferrari et al., 2014) & \\
\hline & & $\begin{array}{c}\text { Minimizar el número } \\
\text { de conexiones entre } \\
\text { zonas }\end{array}$ & (Grayman et al., 2016) & \\
\hline & & $\begin{array}{l}\text { Minimizar contadores } \\
\text { y válvulas de corte }\end{array}$ & $\begin{array}{l}\text { (Di Nardo et al., 2013) } \\
\text { (GIZ \& VAG, 2011) }\end{array}$ & $\begin{array}{l}\text { Reducción de } \\
\text { costes }\end{array}$ \\
\hline & & $\begin{array}{l}\text { Usar VRP y válvulas } \\
\text { antirretorno cerradas } \\
\text { como válvulas } \\
\text { frontera }\end{array}$ & \multirow{2}{*}{$\begin{array}{c}\text { (Sturm \& Thornton, 2005) } \\
\text { (Fanner et al., 2007) }\end{array}$} & \multirow{2}{*}{$\begin{array}{l}\text { Poder usarlas en } \\
\text { caso de incendio } \\
\text { y aumentar la } \\
\text { capacidad }\end{array}$} \\
\hline & & $\begin{array}{l}\text { Instalar VRP en } \\
\text { paralelo al contador } \\
\text { de entrada }\end{array}$ & & \\
\hline
\end{tabular}




\begin{tabular}{|c|c|c|c|c|}
\hline Criterio & Subcriterio & Recomendación & Autor & Comentarios \\
\hline & & $\begin{array}{l}\text { VRP cerradas en } \\
\text { puntos de suministro } \\
\text { secundarios }\end{array}$ & (Fanner et al., 2007) & $\begin{array}{l}\text { La válvula abrirá } \\
\text { cuando las } \\
\text { condiciones de } \\
\text { demanda lo } \\
\text { requieran }\end{array}$ \\
\hline & \multirow[t]{2}{*}{ Diámetro } & $\begin{array}{l}\text { El menor posible para } \\
\text { válvulas de corte }\end{array}$ & $\begin{array}{c}\text { (MacDonald \& Yates, 2005) } \\
\text { (Tzatchkov et al., 2014) } \\
\text { (Fanner et al., 2007) }\end{array}$ & $\begin{array}{l}\text { Minimizar el } \\
\text { efecto final de red }\end{array}$ \\
\hline & & $\begin{array}{l}\text { Tubería de suministro } \\
\qquad>300 \mathrm{~mm}\end{array}$ & (Grayman et al., 2016) & \\
\hline & $\begin{array}{l}\text { Fuentes de } \\
\text { suministro }\end{array}$ & Una por sector & $\begin{array}{l}\text { (Fanner et al., 2007) } \\
\text { (Scarpa et al., 2016) }\end{array}$ & $\begin{array}{l}\text { En ese caso, } \\
\text { denominan a los } \\
\text { sectores i-dma }\end{array}$ \\
\hline & \multirow[b]{2}{*}{$\begin{array}{l}\text { Puntos de } \\
\text { suministro }\end{array}$} & \multirow[b]{2}{*}{ Uno por sector } & (GIZ \& VAG, 2011) & \\
\hline & & & (Gomes et al., 2013) & $\begin{array}{l}\text { Mejor gestión de } \\
\text { esta y menor error } \\
\text { de lectura }\end{array}$ \\
\hline \multirow{3}{*}{ DEMANDA } & \multirow{3}{*}{ Demanda } & $\begin{array}{l}\text { Rango mínimo y } \\
\text { máximos establecido }\end{array}$ & (De Paola et al., 2014) & $\begin{array}{l}\text { Impuesto por } \\
\text { número de } \\
\text { acometidas }\end{array}$ \\
\hline & & $\begin{array}{l}\text { Según la demanda } \\
\text { unitaria y el volumen } \\
\text { consumido }\end{array}$ & (Pesantez et al., 2019) & $\begin{array}{c}\text { Consumo } \\
\text { homogéneo en } \\
\text { todos los sectores }\end{array}$ \\
\hline & & $\begin{array}{l}\text { Ubicar a agrandes } \\
\text { consumidores cerca } \\
\text { de las fronteras o } \\
\text { extremos cerrados }\end{array}$ & $\begin{array}{l}\text { (Sturm \& Thornton, 2005) } \\
\text { (Fanner et al., 2007) }\end{array}$ & $\begin{array}{c}\text { Para evitar } \\
\text { problemas de } \\
\text { estancamiento del } \\
\text { agua }\end{array}$ \\
\hline DISTRIBUCIÓN & $\begin{array}{l}\text { Cotas del } \\
\text { terreno }\end{array}$ & $\begin{array}{l}\text { Distribución } \\
\text { homogénea del } \\
\text { terreno }\end{array}$ & (Thornton, 2003) & $\begin{array}{l}\text { Presión uniforme } \\
\text { en el sector }\end{array}$ \\
\hline COSTES & $\begin{array}{l}\text { Sectorización } \\
\text { de mínimo } \\
\text { coste }\end{array}$ & $\begin{array}{l}\text { Combinación de } \\
\text { robustez y otros } \\
\text { indicadores }\end{array}$ & (Gomes et al., 2013) & $\begin{array}{l}\text { Análisis de } \\
\text { diferentes } \\
\text { escenarios }\end{array}$ \\
\hline
\end{tabular}

Además de todo lo visto hasta ahora, hay otros importantes factores a tener presentes, pero sobre los cuales hay claras recomendaciones establecidas. Los requisitos de redundancia, robustez de la red o el estado de las infraestructuras, son algunos de ellos. Por ello, es de suma importancia abordar, durante el diseño, diferentes posibles escenarios de funcionamiento, tanto presente como futuros (Gomes et al., 2013).

Nótese que, hasta este punto, todos los criterios o recomendaciones han tenido carácter técnico, principalmente hidráulico, quedando al margen las restricciones estrictamente económicas. No obstante, el criterio económico es el que, en la mayoría de los proyectos a realizar, no solo guía la definición final, sino que es el condicionante principal (número máximo de válvulas a instalar, número máximo de caudalímetros a instalar, etc.) (Sturm \& Thornton, 2005). Pocos son los autores que dan un paso más y añaden los criterios económicos a los anteriormente citados. Savić \& Ferrari (2014) y Ferrari \& Savic (2015) defienden que para 
establecer la selección de una u otra sectorización planteada es necesario evaluar los costes y beneficios asociados a cada una de ellas, eligiendo el escenario que proporcione unos mejores comportamientos. $\mathrm{O}$, dicho de otra manera, después de analizar todas las alternativas posibles, la sectorización elegida deberá resultar de realizar un balance hídrico, práctico y económico (Morrison et al., 2007). Gomes et al. (2013) proponen un modelo de optimización que da como resultado una sectorización de mínimo coste y que permite alcanzar un compromiso entre la robustez y los diferentes criterios adoptados para diferentes posibles escenarios futuros.

\subsection{Diseño basado en algoritmos}

Como se ha visto en el apartado anterior, el diseño tradicional de las sectorizaciones no sigue unas guías generales claramente definidas, sino que se basa en la experiencia del diseñador y en procesos de prueba y error que parten de diferentes recomendaciones.

Al margen de esta modalidad generalizada y tradicional de diseño, recientemente han sido publicados nuevos estudios que, abordando el problema desde diferentes teorías o campos de estudio, tienen como objetivo orientar el diseño hacía la minimización del peso de los criterios y procesos empíricos, la experiencia del propio diseñador y plantear procesos autónomos a la hora de la definición de los sectores (Perelman et al., 2014; Perelman et al., 2015; Di Nardo et al., 2015).

La mayor parte de las metodologías están basadas en algoritmos de aprendizaje no supervisados, y aunque cada una de ellas persigue unos objetivos de optimización diferentes, se podrían clasificar en los siguientes tres campos de estudio, según el tipo de representación que haga del sistema de distribución: El primer grupo sería la Teoría de Grafos. Un grafo se representa gráficamente con un conjunto de vértices (nudos) unidos por líneas (tuberías), que permiten representar relaciones entre elementos de un conjunto. El segundo campo de estudio consiste en las Estructuras Comunitarias. Este campo representa a los sistemas de distribución como una red compleja de individuos interconectados entre sí, pero con mayor o menor intensidad según el par de nodos. Y el último campo engloba a los Procesos Heurísticos, que no pueden ser englobados en los dos anteriores. Recogerá todas las metodologías que se centren en la elaboración de principios, estrategias y reglas que faciliten la búsqueda de la vía de solución de un problema dado.

A lo largo de este apartado se entrará en detalle de cada uno de los tres campos de estudio descritos, abordando su adaptación a los sistemas de distribución y cómo, diferentes autores, lo han particularizado para el problema del diseño de la sectorización. Pero antes, y a modo de resumen, en la Tabla 2.4 se recogen los trabajos que mejor representan la metodología elegida y, a su vez, mejores resultados han conseguido o proponen conseguir. Los estudios aparecen ordenados cronológicamente, se cita la base del diseño seguida y notas de este y se indica si la metodología requiere, a priori, el número de sectores a definir o un tamaño de estos y si selecciona o no las tuberías frontera a cerrar para aislar sectores, 
Tabla 2.4 Nuevas metodologías recogidas en la bibliografía sobre la sectorización

\begin{tabular}{|c|c|c|c|c|c|c|c|}
\hline Autores & Año & Artículo & Base del diseño & $\begin{array}{l}\text { ¿Necesita } \\
\text { número de } \\
\text { sectores? }\end{array}$ & $\begin{array}{l}\text { ¿Necesita } \\
\text { tamaño de } \\
\text { sectores? }\end{array}$ & $\begin{array}{l}\text { Propone } \\
\text { tuberías } \\
\text { de corte }\end{array}$ & Notas \\
\hline Hunaidi, 2005 & 2005 & $\begin{array}{c}\text { Economic comparison of periodic acoustic } \\
\text { surveys and DMA-based leakage } \\
\text { management strategies }\end{array}$ & $\begin{array}{l}\text { Costes de búsqueda } \\
\text { de fugas }\end{array}$ & Si & No & No & \\
\hline $\begin{array}{l}\text { Tzatchkov et al., } \\
2008\end{array}$ & 2008 & $\begin{array}{l}\text { Graph theory-based algorithms for water } \\
\text { distribution network sectorization projects }\end{array}$ & Teoría de Grafos & $\mathrm{Si}$ & $\mathrm{Si}$ & No & \\
\hline $\begin{array}{l}\text { Di Nardo \& Di Natale, } \\
\qquad 2010\end{array}$ & 2010 & $\begin{array}{l}\text { A design support methodology for district } \\
\text { metering of water supply networks }\end{array}$ & Teoría de Grafos & $\mathrm{Si}$ & $\mathrm{Si}$ & Sí & $\begin{array}{l}\text { Propone ubicaciones para } \\
\text { contadores y válvulas de } \\
\text { cortes. Es necesario } \\
\text { verificar con Epanet y no } \\
\text { busca un óptimo de algún } \\
\text { tipo. }\end{array}$ \\
\hline Herrera et al., 2010 & 2010 & $\begin{array}{l}\text { Water supply clusters by multi-agent-based } \\
\text { approach }\end{array}$ & Multi-Agentes & No & Si & No & \\
\hline $\begin{array}{l}\text { Di Nardo \& Di Natale, } \\
\qquad 2011\end{array}$ & 2011 & $\begin{array}{l}\text { A heuristic design support methodology } \\
\text { based on graph theory for district metering of } \\
\text { water supply networks. }\end{array}$ & Teoría de Grafos & $\mathrm{Si}$ & $\mathrm{Si}$ & No & \\
\hline Di Nardo et al., 2011 & 2011 & $\begin{array}{c}\text { Graph partitioning for automatic sectorization } \\
\text { of a water distribution system. }\end{array}$ & Partición de grafos & & & & \\
\hline Gomes et al., 2012 & 2012 & $\begin{array}{l}\text { Decision support system to divide a large } \\
\text { network into suitable District Metered Areas }\end{array}$ & & Si & No & Sí & \\
\hline Tzatchkov et al., 2012 & 2012 & $\begin{array}{l}\text { Graph partitioning algorithms for water } \\
\text { distribution network sectorization projects. }\end{array}$ & Teoría de Grafos & $\mathrm{Si}$ & $\mathrm{Si}$ & No & $\begin{array}{l}\text { Emplea los algoritmos de } \\
\text { partición. }\end{array}$ \\
\hline Ferrari et al., 2014 & 2013 & $\begin{array}{c}\text { Graph-Theoretic Approach and Sound } \\
\text { Engineering Principles for Design of District } \\
\text { Metered Areas }\end{array}$ & $\begin{array}{l}\text { Teoría de Grafos y } \\
\text { algoritmo de } \\
\text { bipartición }\end{array}$ & No & No & No & - \\
\hline
\end{tabular}




\begin{tabular}{|c|c|c|c|c|c|c|c|}
\hline Autores & Año & Artículo & Base del diseño & $\begin{array}{l}\text { ¿Necesita } \\
\text { número de } \\
\text { sectores? }\end{array}$ & $\begin{array}{l}\text { ¿Necesita } \\
\text { tamaño de } \\
\text { sectores? }\end{array}$ & $\begin{array}{l}\text { Propone } \\
\text { tuberías } \\
\text { de corte }\end{array}$ & Notas \\
\hline Diao et al., 2013 & 2013 & $\begin{array}{l}\text { Automated creation of district metered area } \\
\text { boundaries in water distribution systems }\end{array}$ & $\begin{array}{l}\text { Agrupación por } \\
\text { comunidades } \\
\text { (Radicchi, 2004) }\end{array}$ & $\mathrm{Si}$ & $\mathrm{Si}$ & No & $\begin{array}{l}\text { Esta metodología sí que } \\
\text { obtiene una primera } \\
\text { aproximación de las } \\
\text { tuberías que hay que } \\
\text { cortar. }\end{array}$ \\
\hline Di Nardo et al., 2013b & 2013 & $\begin{array}{c}\text { Water Network Sectorization based on } \\
\text { genetic algorithm and minimum dissipated } \\
\text { power paths }\end{array}$ & $\begin{array}{l}\text { Teoría de Grafos y } \\
\text { algoritmos genéticos }\end{array}$ & No & $\mathrm{Si}$ & Sí & $\begin{array}{l}\text { Busca sectores con } \\
\text { suministro independientes } \\
\text { al resto (iDMA) }\end{array}$ \\
\hline Scibetta et al., 2014 & 2014 & $\begin{array}{c}\text { Community Detection as a Tool for District } \\
\text { Metered Areas Identification }\end{array}$ & $\begin{array}{l}\text { Agrupación por } \\
\text { comunidades }\end{array}$ & $\mathrm{Si}$ & $\mathrm{Si}$ & No & \\
\hline De Paola et al., 2014 & 2014 & $\begin{array}{l}\text { Automatic multi-objective sectorization of a } \\
\text { water distribution network. }\end{array}$ & $\begin{array}{l}\text { Teoría de Grafos y } \\
\text { algoritmos genéticos }\end{array}$ & $\mathrm{Si}$ & No & No & \\
\hline Di Nardo et al., 2014 & 2014 & $\begin{array}{c}\text { Ant algorithm for smart water network } \\
\text { partitioning }\end{array}$ & $\begin{array}{l}\text { Teoría de Grafos y } \\
\text { algoritmos genéticos }\end{array}$ & $\mathrm{Si}$ & $\mathrm{Si}$ & Sí & $\begin{array}{c}\text { Propone ubicaciones para } \\
\text { contadores y válvulas de } \\
\text { cortes. Es necesario } \\
\text { verificar con Epanet y no } \\
\text { busca un óptimo de algún } \\
\text { tipo. }\end{array}$ \\
\hline $\begin{array}{l}\text { Giustolisi \& Ridolfi, } \\
2014\end{array}$ & 2014 & $\begin{array}{c}\text { Modularity Index for Hydraulic System } \\
\text { Segmentation. }\end{array}$ & $\begin{array}{l}\text { Agrupación por } \\
\text { comunidades }\end{array}$ & $\mathrm{Si}$ & $\mathrm{Si}$ & No & $\begin{array}{l}\text { Se trata de un proceso } \\
\text { iterativo. }\end{array}$ \\
\hline $\begin{array}{l}\text { Castro Gama et al., } \\
\qquad 2014\end{array}$ & 2014 & $\begin{array}{c}\text { Model-Based Sectorization of Water } \\
\text { Distribution Networks For Increased Energy } \\
\text { Efficiency }\end{array}$ & $\begin{array}{l}\text { Teoría de Grafos y } \\
\text { algoritmos genéticos }\end{array}$ & $\mathrm{Si}$ & $\mathrm{Si}$ & Sí & $\begin{array}{c}\text { Esta metodología sí que } \\
\text { obtiene una primera } \\
\text { aproximación de las } \\
\text { tuberías que hay que } \\
\text { cortar. }\end{array}$ \\
\hline Di Nardo et al., 2015 & 2015 & $\begin{array}{l}\text { Software for partitioning and protecting a } \\
\text { water supply network }\end{array}$ & $\begin{array}{l}\text { Teoría de Grafos y } \\
\text { algoritmos genéticos }\end{array}$ & $\mathrm{Si}$ & $\mathrm{Si}$ & Sí & $\begin{array}{l}\text { Propone ubicaciones para } \\
\text { contadores y válvulas de } \\
\text { cortes. Es necesario } \\
\text { verificar con Epanet. }\end{array}$ \\
\hline
\end{tabular}




\begin{tabular}{|c|c|c|c|c|c|c|c|}
\hline Autores & Año & Artículo & Base del diseño & $\begin{array}{l}\text { ¿Necesita } \\
\text { número de } \\
\text { sectores? }\end{array}$ & $\begin{array}{l}\text { ¿Necesita } \\
\text { tamaño de } \\
\text { sectores? }\end{array}$ & $\begin{array}{l}\text { Propone } \\
\text { tuberías } \\
\text { de corte }\end{array}$ & Notas \\
\hline Giustolisi et al., 2015 & 2015 & $\begin{array}{c}\text { General metrics for segmenting infrastructure } \\
\text { networks }\end{array}$ & $\begin{array}{l}\text { Estructuras } \\
\text { comunitarias }\end{array}$ & $\mathrm{Si}$ & $\mathrm{Si}$ & No & \\
\hline Scarpa et al., 2016 & 2016 & $\begin{array}{l}\text { Elementary DMA Design of Looped Water } \\
\text { Distribution Networks with Multiple Sources }\end{array}$ & Teoría de Grafos & No & $\mathrm{Si}$ & No & $\begin{array}{l}\text { Propone un sector por } \\
\text { cada punto de suministro } \\
\text { (depósito). El cierre de } \\
\text { tuberías lo selecciona con } \\
\text { un algoritmo de prueba y } \\
\text { error. }\end{array}$ \\
\hline Korkana et al., 2016 & 2016 & $\begin{array}{l}\text { Developing an optimization algorithm to form } \\
\text { district metered areas in a water distribution } \\
\text { system }\end{array}$ & Proceso heurístico & No & No & $\mathrm{Si}$ & $\begin{array}{l}\text { Algoritmo de combinación } \\
\text { de tuberías cerradas y } \\
\text { análisis hidráulico de cada } \\
\text { escenario. }\end{array}$ \\
\hline Di Nardo et al., 2016 & 2016 & $\begin{array}{l}\text { Dynamic control of water distribution system } \\
\text { based on network partitioning }\end{array}$ & $\begin{array}{l}\text { Teoría de Grafos y } \\
\text { Proceso heurístico }\end{array}$ & No & No & $\mathrm{Si}$ & $\begin{array}{l}\text { Optimización del número } \\
\text { de tuberías a cerrar. }\end{array}$ \\
\hline Brentan et al., 2017 & 2017 & $\begin{array}{c}\text { Social network community detection for DMA } \\
\text { creation: criteria analysis through multilevel } \\
\text { optimization. }\end{array}$ & $\begin{array}{c}\text { Estructuras } \\
\text { comunitarias y } \\
\text { Proceso heurístico }\end{array}$ & $\mathrm{Si}$ & No & Si & \\
\hline Di Nardo et al., 2017 & 2017 & $\begin{array}{c}\text { Economic and energy criteria for district } \\
\text { meter areas design of water distribution } \\
\text { networks. }\end{array}$ & $\begin{array}{l}\text { Teoría de Grafos y } \\
\text { Proceso heurístico }\end{array}$ & Si & No & $\mathrm{Si}$ & \\
\hline Laucelli et al., 2017 & 2017 & $\begin{array}{l}\text { Optimal design of district metering areas for } \\
\text { the reduction of leakages. }\end{array}$ & $\begin{array}{c}\text { Estructuras } \\
\text { comunitarias y } \\
\text { Proceso heurístico }\end{array}$ & $\mathrm{Si}$ & $\mathrm{Si}$ & $\mathrm{Si}$ & \\
\hline Zhang et al., 2017 & 2017 & $\begin{array}{l}\text { Automatic partitioning of water distribution } \\
\text { networks using multiscale community } \\
\text { detection and multiobjective optimization. }\end{array}$ & $\begin{array}{c}\text { Estructuras } \\
\text { comunitarias y } \\
\text { Proceso heurístico }\end{array}$ & $\mathrm{Si}$ & $\mathrm{Si}$ & $\mathrm{Si}$ & $\begin{array}{l}\text { La función objetivo para la } \\
\text { creación de los sectores } \\
\text { depende del número de } \\
\text { tuberías a cerrar y una } \\
\text { presión y tiempo de } \\
\text { permanencia uniformes. }\end{array}$ \\
\hline
\end{tabular}




\begin{tabular}{|c|c|c|c|c|c|c|c|}
\hline Autores & Año & Artículo & Base del diseño & $\begin{array}{l}\text { ¿Necesita } \\
\text { número de } \\
\text { sectores? }\end{array}$ & $\begin{array}{l}\text { ¿Necesita } \\
\text { tamaño de } \\
\text { sectores? }\end{array}$ & $\begin{array}{l}\text { Propone } \\
\text { tuberías } \\
\text { de corte }\end{array}$ & Notas \\
\hline $\begin{array}{l}\text { Chatzivasili et al., } \\
\qquad 2019\end{array}$ & 2019 & $\begin{array}{c}\text { Optimizing the Formation of DMAs in a Water } \\
\text { Distribution Network through Advanced } \\
\text { Modelling }\end{array}$ & $\begin{array}{l}\text { Teoría de Grafos y } \\
\text { Proceso heurístico }\end{array}$ & $\mathrm{Si}$ & $\mathrm{Si}$ & $\mathrm{Si}$ & $\begin{array}{l}\text { La función objetivo para la } \\
\text { creación de los sectores } \\
\text { depende del tiempo de } \\
\text { permanencia del agua y } \\
\text { del producto } \\
\text { presión.demanda. }\end{array}$ \\
\hline Pesantez et al., 2019 & 2019 & $\begin{array}{c}\text { Multiphase Procedure to Design District } \\
\text { Metered Areas for Water Distribution } \\
\text { Networks }\end{array}$ & $\begin{array}{l}\text { Teoría de Grafos y } \\
\text { Proceso heurístico }\end{array}$ & $\mathrm{Si}$ & $\mathrm{Si}$ & $\mathrm{Si}$ & $\begin{array}{c}\text { Emplea variables } \\
\text { hidráulicas para el proceso } \\
\text { de optimización, pero } \\
\text { siempre buscando } \\
\text { sectores son demandas } \\
\text { totales similares. }\end{array}$ \\
\hline
\end{tabular}




\subsubsection{Teoría de grafos}

Un grafo es una estructura formada por un conjunto de vértices, unidos por unos enlaces llamados aristas o arcos. Así, se suelen representar gráficamente como un conjunto de puntos (vértices) unidos por líneas (aristas) (Schaeffer, 2007). Los grafos son una herramienta para mostrar la relación entre diferentes elementos de un conjunto. Su estudio queda englobado dentro de la Teoría de Grafos, campo de estudio muy maduro que presenta multitud de propiedades, características y cualidades muy desarrolladas para diferentes enfoques y campos de aplicación.

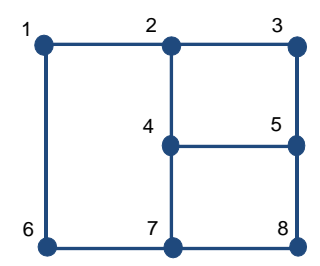

$$
A=\left[\begin{array}{llllllll}
1 & 1 & 0 & 0 & 0 & 1 & 0 & 0 \\
1 & 1 & 1 & 1 & 0 & 0 & 0 & 0 \\
0 & 1 & 1 & 0 & 1 & 0 & 0 & 0 \\
0 & 1 & 0 & 1 & 1 & 0 & 1 & 0 \\
0 & 0 & 1 & 1 & 1 & 0 & 0 & 1 \\
1 & 0 & 0 & 0 & 0 & 1 & 1 & 0 \\
0 & 0 & 0 & 1 & 0 & 1 & 1 & 1 \\
0 & 0 & 0 & 0 & 1 & 0 & 1 & 1
\end{array}\right]
$$

Figura 2.3. Ejemplo de Grafo (izq.) y su matriz de Adyacencias (der.)

Los grafos pueden ser representados de manera gráfica, analítica o matricial. La representación gráfica, Figura 2.3, es la forma más sencilla de observar un grafo, y la relación y disposición de sus elementos. La representación analítica presenta al grafo $G$ de manera más formal. Así, dicho grafo tiene las siguientes características:

$$
\left\{\begin{array}{c}
G=(V, E) \\
V=(1,2 \ldots n) \\
(i, j) \in E \leftrightarrow a_{i j} \neq 0
\end{array}\right.
$$

donde $V$ es el conjunto de los $n$ vértices y $E$ el de las aristas del grafo.

La última forma de representar un grafo es algebraicamente, usando para ello matrices cuadradas simétricas. Estas matrices reciben el nombre de matriz de adyacencias (Figura 2.3), y están compuestas por valores booleanos, que adquieren el valor de 1 siempre y cuando el elemento de la fila i está conectado con el elemento de la fila j mediante una arista, en caso contrario el elemento recibe el valor 0.

Los vértices y aristas de los grafos pueden estar etiquetados, con cualquier valor o cualidad. Por ejemplo, las líneas de un grafo pueden tener asignada una longitud, en el caso de representar rutas de transporte, y los nudos la densidad de población de la ciudad que representen. Incluso, sin mayor problema, se pueden asociar propiedades hidráulicas de un sistema de distribución de agua. Esto, junto con el hecho de la similitud entre un grafo y la topología de las redes de distribución de agua (Figura 2.4), donde las tuberías son las líneas y los nudos los puntos de consumo, ha hecho que numerosos autores aborden a estas infraestructuras como grafos, con el objetivo de dotar a los sistemas de distribución de las ventajas que brinda la Teoría de Grafos. Por citar algún caso, Bartolín (2013) aplica esta semejanza para buscar caminos mínimos desde los puntos de suministro hasta los puntos de consumo. 


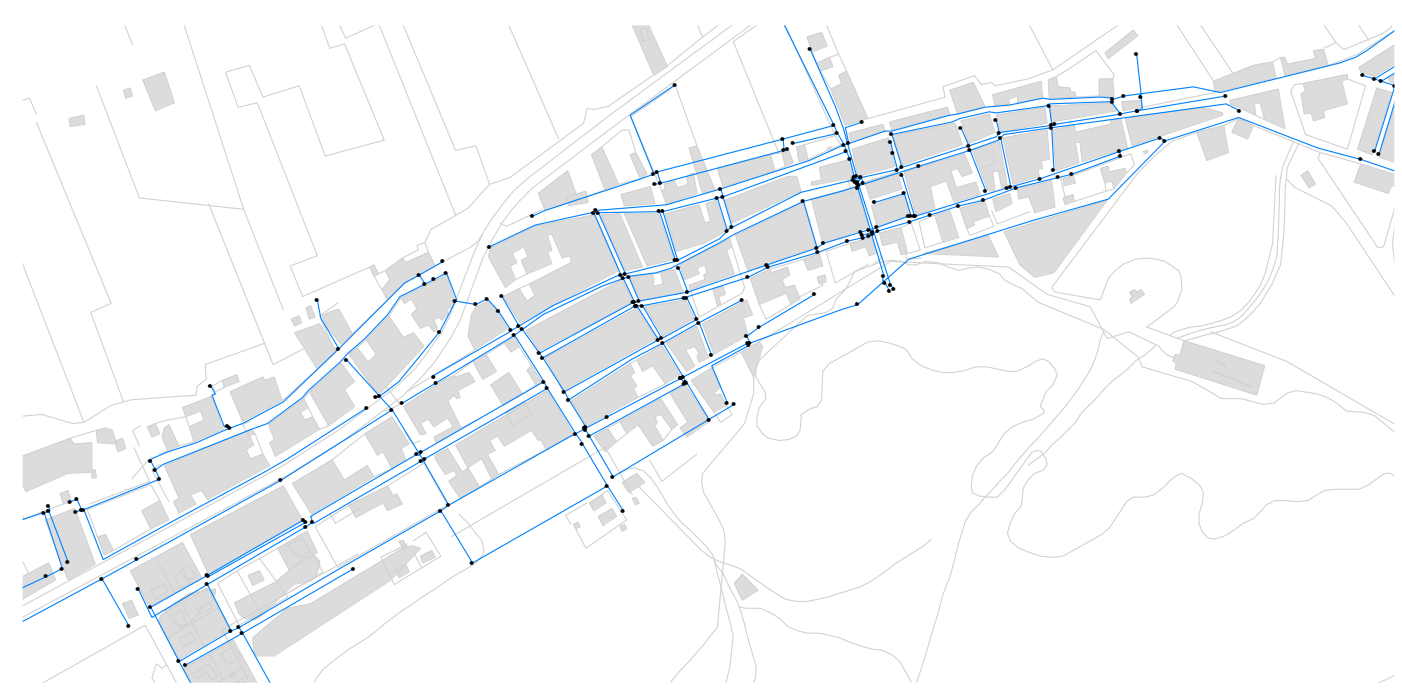

Figura 2.4. Vista en planta de una red de distribución

\subsubsection{Principales usos de los grafos}

Las aplicaciones de los grafos son muy extensas, pero entre todas ellas, el estudio de la conectividad se podría citar como una de las más extendidas. Así, se pueden destacar los siguientes algoritmos de búsqueda:

- Búsqueda de profundidad (DFS o Depth First Search): El DFS es un algoritmo utilizado para recorrer todos los nudos de un grafo de manera ordenada, pero no uniforme. El proceso comienza en un nudo y realiza una expansión hacía nuevos caminos, hasta que no quedan más nudos por visitar. En ese caso, regresa al último camino posible y repite el mismo proceso (Figura 2.5). Este algoritmo puede ser útil, dentro de los sistemas de distribución de agua, a la hora de conocer el camino más corto entre un punto de suministro y uno de consumo, o identificar aquellas zonas independientes durante la fase de construcción de un modelo de simulación hidráulica (Vegas et al., 2016).

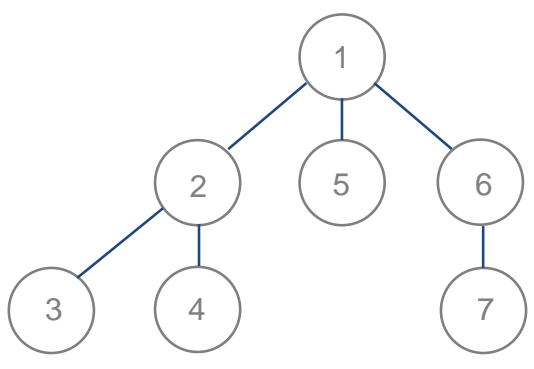

Figura 2.5. Pasos de búsqueda seguidos con el DFS

- Búsqueda en anchura (BFS o Breadth First Search): Al igual que el anterior, se trata de un algoritmo empleado para recorrer todos los nudos del grafo, pero el proceso seguido es distinto. En este caso, el algoritmo recorre el grafo en frentes de vértices y explora todos los nudos vecinos al nudo en que se encuentra. Paso seguido, para cada uno de los vecinos, se exploran sus respectivos vecinos adyacentes y así hasta recorrer todo el grafo. Un ejemplo de aplicación del BFS dentro de los sistemas de distribución de agua, podría ser el obtener una primera aproximación de las zonas suministradas por cada punto de producción. 


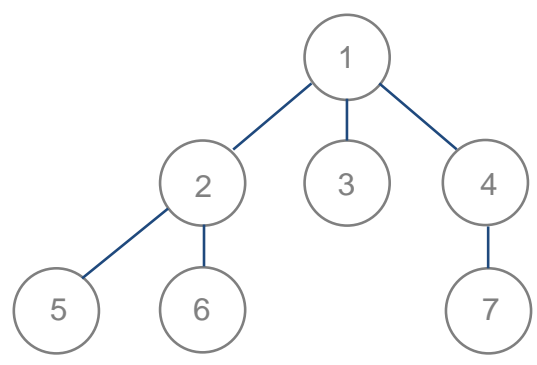

Figura 2.6. Pasos de búsqueda seguidos con el BFS

- Algoritmo de Dijkstra: El algoritmo planteado por Dijkstra (1959), también conocido como algoritmo de caminos mínimos, obtiene el camino mínimo más corto entre un origen y el resto de los vértices en un grafo dirigido con pesos en cada arista.

Este algoritmo parte de un nudo dado y explora los posibles caminos a tomar, calcula el peso de todos ellos y elige el que tenga menor peso. Este algoritmo es similar al formulada por Prim (1957), con el cual se encuentra un árbol recubridor mínimo en un grafo conexo, no dirigido y cuyas aristas están etiquetadas. A diferencia de los anteriores algoritmos, el camino seguido por este método vendrá impuesto por los valores de los elementos seleccionados, y no únicamente por la topología de la red.

\subsubsection{Aplicación en la sectorización de redes de distribución de agua}

Como se ha comentado al principio de este apartado, son muchos los autores que han aplicado la teoría de grafos en la gestión de los sistemas de distribución de agua. Para el caso concreto que nos ocupa, a continuación, se detallan aplicaciones propuestas por diferentes autores con el objetivo de mejorar la forma de diseño y gestión de esta técnica:

- Análisis de conectividad: Deuerlein (2008) aborda el concepto de la red como un gráfico con propiedades de conectividad, lo cual le permitía diferenciar en la red una parte exterior, a la que denominaría bosque, y una central o interior, la cual quedaría definida como núcleo. Simplificación esta que podría ayudar a desarrollar estrategias más eficientes de operación y control, según afirma el autor mencionado. Perelman \& Ostfeld (2011) plantean un algoritmo basado en búsquedas en profundidad y búsquedas en anchura que divide el sistema en diferentes grupos según la dirección del flujo en las tuberías, pero sin considerar el comportamiento hidráulico del sistema. Para ello habría que recurrir a la metodología presentada por Di Nardo \& Di Natale (2011), que, considerando los caudales y presiones de la red, calcula todos los caminos de mínima pérdida entre cada punto de distribución y cada punto de consumo, y su frecuencia de paso. Obtiene como primer resultado un gráfico principal y una primera definición de la sectorización. A partir de aquí, el diseñador deberá seleccionar qué tuberías deberá cerrar para aislar los sectores o dónde colocar los contadores, siempre bajo la previa comprobación hidráulica de las alternativas seleccionadas. Para solventar esta desventaja, posteriormente Di Nardo et al. (2013b) complementarán la anterior metodología con un algoritmo genético para determinar la frontera entre los diferentes sectores. En este caso, los autores partirían de afirmar que existe un grupo de nudos que se encuentran en los caminos de mínima pérdida desde varios depósitos. Por ello proponen, con la ayuda de algoritmos genéticos, ir moviendo de sectores estos nudos según la siguiente función objetivo: 


$$
\text { F.O. } \min \frac{\text { núnero de nudos }}{\sum_{i}^{n} h_{i}}
$$

donde $n$ es el número de nudos y $h_{i}$ es la altura piezométrica del nudo $i$.

Un estudio similar (Gomes et al., 2012) presenta una metodología que emplea el algoritmo Floyd-Warshall para identificar el camino con menor peso, con relación a caudales y diámetros, entre cada punto de suministro y nudo de consumo. Más recientemente, y realizando un estudio parecido al anterior, Alvisi \& Franchini (2014) proponen un procedimiento de sectorización basado en el algoritmo de búsqueda en profundidad y el del camino más corto. Pero a diferencia de los anteriores, el procedimiento de cálculo empieza en un nudo al azar y se va extendiendo por la red hasta alcanzar un tamaño máximo establecido con anterioridad.

- Zonas de influencia y contribución de las fuentes de suministro: Otros autores sectorizan la red priorizando la seguridad frente a posibles contaminaciones 0 calidades del agua. En este sentido, algunos autores buscan crear sectores independientes y abastecidos desde diferentes puntos de suministro. Di Nardo et al. (2013a) definen los sectores i-DMA (isolated District Metered Area) como aquellas zonas que se abastecen desde un punto de suministro que se encuentra en su interior o únicamente le suministra a ella, pudiendo quedar totalmente independiente del resto. Con el mismo objetivo, Scarpa et al. (2016) presentan una metodología para seleccionar y diseñar sectores independientes que se suministren desde fuentes diferentes, basándose, en este caso, en el caudal y sentido del flujo en las tuberías. Evidentemente, ambas metodologías definirán tantos sectores como puntos de suministro existan.

- Algoritmos de partición: El último grupo de las metodologías que se apoyan principalmente en la teoría de grafos son los que emplean algoritmos de partición. Con ello consiguen partir el grafo inicial en $k$ subgrafos, los cuales representarán sectores independientes. Existen multitud de algoritmos de partición y autores que los emplean para sectorizar las redes. Di Nardo et al. (2011) plantean una metodología basada en el método de bisección recursiva multinivel (Multilevel Recursive Bisection, MLRB) planteando la partición de redes bajo cuatro escenarios: sin pesos asociados, asociando un peso a las líneas (caudal, pérdida de carga o diámetro), asociando un peso a los nudos (consumo) o una combinación de las anteriores.

Tzatchkov et al. (2012) proponen un algoritmo de partición en función de la distancia desde el punto de suministro al nudo y de la pérdida de carga en el recorrido.

Posteriormente, Ferrari et al. (2014) emplean la misma técnica que Di Nardo et al. (2011) pero estableciendo unos consumos por sector. Chatzivasili et al. (2019) emplean la técnica de partición conocida como Recursive Coordinate Bisection (RCB) para la división de las redes.

\subsubsection{Estructuras comunitarias}

Una comunidad se puede definir como un grupo de elementos que tienen ciertas cualidades en común. Si, además, estos elementos pueden ser agrupados en grupos, entonces estos forman una estructura comunitaria (Girvan \& Newman, 2002; Newman, 2004). En el contexto de las redes, la estructura comunitaria se refiere a la ocurrencia de grupos cuyos elementos interactúan más fuertemente entre sí que con los elementos de grupos vecinos. 

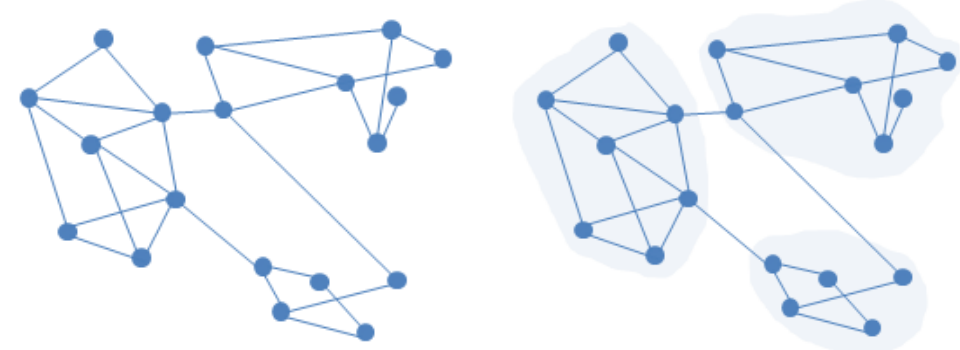

Figura 2.7. Estructura comunitaria (izquierda) formada por 3 comunidades (derecha).

La estructura comunitaria (Figura 2.7) es, para Girvan \& Newman (2002), una propiedad de las redes, las cuales quedan definidas por las líneas de intermediación o interconexión. Y su estudio es una modalidad madura debido al gran ámbito de aplicación que tiene (redes naturales, sociales, tecnológicas, etc.), con numerosos algoritmos y teorías para su definición y detección (Newman, 2004). De todos los indicadores empleados por los autores para ello, habría que destacar el concepto de modularidad definido por Newman \& Girvan (2003). La modularidad mide la fuerza de agrupación o conexión que existe dentro de la estructura comunitaria, y queda definida según la ecuación 2.3:

$$
Q=\sum_{i=1}^{c}\left(e_{i i}-a_{i}^{2}\right)
$$

donde $e_{i i}$ representa la fracción de aristas que conectan dos vértices de la misma comunidad, siendo un valor alto si se tienen comunidades claras o bien definidas, $c$ es el número de comunidades y $a_{i}$ representa la fracción de tuberías que conectan un vértice de otra comunidad $j$ con uno de la comunidad $i$, quedando definida por la ecuación 2.4 :

$$
a_{i}=\sum_{i=1}^{c} e_{i j}
$$

Los sistemas de distribución de agua también se pueden representar como una estructura comunitaria. Para ello, tan solo es necesario fijar los puntos de consumo como los elementos de la comunidad, que se encuentran conectados por las tuberías, y que tienen conexiones más o menos fuertes según la distribución de las infraestructuras. Este punto de vista, el de una red de distribución de agua potable como una estructura comunitaria, ha sido abordado por varios autores en sus trabajos atraídos por la necesidad de simplificar la red, y así las tareas de análisis y gestión.

En este sentido, Girvan \& Newman (2002) plantean detectar estas comunidades dentro de las redes, empleando el concepto edge betweennes (tuberías de intermediación/interconexión), con el cual consiguen destacar las zonas aisladas. Diao et al. (2013) estudian agrupar todos los elementos de tal forma que la densidad de unión entre ellos sea mayor que con el resto de los elementos de otras comunidades, empleando para ello la modularidad (ecuación 2.3). Nuevamente, estos autores son capaces de detectar grupos y delimitar las conexiones entre ellos, sin llegar a estudiar si deben cerrarse o no. De forma similar, pero dando un paso más, Scibetta et al. (2014) reconocen el grado de afinidad entre los nudos de una red y los engloba en diferentes sectores basándose en la modularidad, el flujo y el caudal de las diferentes tuberías. Sobre estos mismos conceptos, pero añadiendo a cada línea un peso y el coste económico de la implantación de la sectorización y el uso de algoritmos genéticos, Giustolisi 
\& Ridolfi (2014) consiguen plantear un frente de pareto con soluciones al problema de la sectorización planteado.

\subsubsection{Principales usos de las estructuras comunitarias}

Dentro de las estructuras comunitarias, las dos tareas de aprendizaje no supervisadas más empleadas son la agrupación de los datos en grupos de similitud (Clustering) y la reducción de la dimensionalidad para comprimir los datos mientras se mantienen su estructura y funcionalidad.

La primera de ellas, tiene como objetivo organizar todos los nudos en diferentes grupos según una similitud definida. Con este objetivo, se han definido varias metodologías de agrupación, de las cuales se pueden destacar las siguientes:

- Agrupación k-means: Es un algoritmo de agrupación de los elementos en $k$ grupos, de tal forma que los individuos de un grupo estén mejor relacionados entre sí que con el resto de los vecinos. La forma que tiene este algoritmo de agrupar es la siguiente:

1. Se seleccionan los $k$ puntos que, inicialmente, serán los centroides de cada grupo.

2. Cada elemento se clasifica calculando su distancia con cada centro de grupo, y se incluye en el grupo cuyo centro esté más cerca de él.

3. Según los elementos agrupados, y tomando la media de todos los vectores en el grupo, se recalcula el centro del conjunto.

4. Se repiten los pasos 2 y 3 hasta que los centros de grupo no cambien mucho entre iteraciones o un número determinado de veces.

Por otro lado, k-means presenta un par de desventajas. Por un lado, el número de grupos es necesario definirlo. Esto no siempre es trivial y puede resultar en soluciones no óptimas según los datos a agrupar. Además, en esta agrupación pueden producirse diferentes resultados en diferentes ejecuciones del algoritmo debido a que los centros de agrupación se seleccionan de manera aleatoria.

- Agrupación de desplazamiento medio: La agrupación de desplazamiento medio se basa en ventanas deslizantes que pretenden localizar áreas densas de puntos de datos. El objetivo de este algoritmo es ubicar los puntos centrales de cada grupo/clase. La ventaja de este algoritmo, frente a la agrupación de k-means, reside en que no es necesario definir el número de grupos, ya que estos se crean automáticamente. En cambio, la desventaja de este método está en que la selección del tamaño de la ventana puede no ser trivial.

- Agrupamiento espacial basado en densidad de aplicaciones con ruido (Density-Based Spatial Clustering of Applications with Noise, DBSCAN): Es uno de los algoritmos más usados y citado en la literatura científica. Basa su agrupación en la densidad de los grupos, y presenta grandes ventajas frente a otras técnicas similares. Principalmente, no es necesario fijar el número de grupos. Además, es capaz de detectar correctamente aquellos nudos con valores atípicos, así como grupos de tamaño y forma arbitraria. En contrapartida, ante grupos con densidad variable el DBSCAN no funciona perfectamente. 
- Agrupamiento jerárquico (Hierarchical clustering): La agrupación jerárquica es similar a la agrupación regular, excepto que su objetivo es crear una jerarquía de agrupaciones. Se aconseja su empleo en aquellos datos cuya estructura es jerárquica, y puede ser útil cuando se requiere flexibilidad en la cantidad de clústeres que finalmente se desea. En términos de resultados del algoritmo, además de las asignaciones de clúster, también construye un árbol que informa sobre las jerarquías entre los clústeres. Luego se puede elegir el número de grupos que se desea de este árbol. Por lo que el número de agrupaciones no es necesario especificarlo.

Como ventaja adicionar, la métrica de distancia seleccionada no es crítica ya que no afecta al resultado final, cosa que no pasa en otros algoritmos similares.

La segunda tarea, la reducción de la dimensionalidad, es muy similar a las técnicas de compresión y trata de reducir la complejidad de los datos mientras se mantiene la mayor cantidad de estructura posible. A diferencia de la técnica de agrupamiento, esta metodología no es sencilla y, por ello, han sido pocos los autores que han recurrido a ella para abordar el diseño de las sectorizaciones. Un ejemplo de ello es Herrera et al. (2012). Al igual que en el caso anterior, a continuación se citan dos de las metodologías de reducción más comúnmente empleadas:

- Análisis de componentes principales (Principal Component Analysis, PCA): Se trata de una técnica cuyo objetivo es reducir la dimensionalidad de un conjunto de datos. Para ello, representa los datos en aquella proyección donde estén mejor representados, en términos de mínimos cuadrados. Se consigue así, transformar un conjunto de observaciones de variables en un conjunto de valores de variables sin correlación líneal, llamadas componentes principales.

- Descomposición en valores singulares (Singular Value Descoposition, SVD): EI SVD es un método apropiado para aquellos conjuntos de datos que pueden ser representados de manera vectorial o matricial. $Y$ trata de descomponer la matriz original en la suma de tres matrices, de tal forma que sea posible descomponer los vectores de los datos en ejes ortogonales.

\subsubsection{Aplicación en la sectorización de redes}

Diao et al. (2013) presentan una metodología que consiste en agrupar todos los elementos de la red en diferentes sectores, buscando una maximización de la modularidad del sistema completo, según la siguiente expresión:

$$
Q=\frac{1}{2 m} \sum_{i j}\left[a_{i j}-\frac{k_{i} \cdot k_{j}}{2 m}\right] \cdot \delta\left(C_{i}, C_{j}\right)
$$

donde $a_{i j}$ es el elemento ij de la matriz $\mathrm{A}_{12}, m$ el número total de líneas, $k_{i, j}$ corresponde con el grado del nudo $i$ o $j$, o lo que es lo mismo, el número de líneas que inciden sobre ese nudo, $\delta\left(C_{i}, C_{j}\right)$ adquiere un valor de 1 si los nudos $i$ y $j$ pertenecen a la misma comunidad y 0 en caso contrario.

Una vez planteado el agrupamiento, y calculado el valor de la modulación, es necesario seleccionar qué tuberías se quieren cerrar para delimitar los diferentes sectores y comprobar su funcionamiento hidráulico. 
En línea de la metodología anterior, Scibetta et al. (2014) presentan una metodología para encontrar la frontera de los sectores rápidamente con la ayuda de un algoritmo, el cual consiste en ir moviendo los nudos entre diferentes sectores y evaluar la modularidad del sistema. Con la diferencia de que consideran, en su cálculo, la dirección y cantidad del flujo que circula por las tuberías. Así, los autores emplean la siguiente expresión:

$$
Q=\frac{1}{W} \sum_{i j}\left[w_{i j}-\frac{S_{i}^{\text {out }} \cdot S_{j}^{i n}}{2 m}\right] \cdot \delta\left(C_{i}, C_{j}\right)
$$

donde $w_{i j}$ corresponde con el peso de la línea que une los nudos $i$ y $j$, y que los autores consideran como el caudal circulante, $W$ es la suma de todos los pesos, $s_{i}^{\text {out }}$ corresponde con la suma de los pesos salientes del nudo $i$, y se calcula como $s_{i}^{\text {out }}=\sum_{j} w_{i j}$, $s_{j}^{\text {in }}$ corresponden con la suma de los pesos entrantes al nudo $j$, y se calcula como $s_{j}^{i n}=\sum_{i} w_{i j}$ y el término $\delta\left(C_{i}, C_{j}\right)$ adquiere un valor de 1 si los nudos $i$ y $j$ pertenecen a la misma comunidad y 0 en caso contrario.

En último lugar, Giustolisi \& Ridolfi (2014) presentan una estrategia multi-objetivo para una óptima segmentación de una red, buscando maximizar la modularidad y minimizar el coste asociado al cierre de las tuberías para aislar los sectores, según las siguientes expresiones:

$$
\left\{\begin{array}{c}
\max (Q)=\max \left(1-\frac{n_{c}}{n_{p}}-\sum_{m=1}^{n_{m}}\left(\sum_{k=1}^{n_{p}} \frac{\left(w_{p}\right)_{k} \cdot \delta\left(M_{m}, M_{k}\right)}{W}\right)^{2}\right) \\
\min (\text { cost })=\min \left(\sum_{j=1}^{n_{d}} c_{j}\right)
\end{array}\right.
$$

donde $n_{c}$ es el número de líneas de conexión entre sectores, $n_{p}$ es el número de líneas, $n_{m}$ el número de sectores, $W_{p}$ es peso de la línea (los autores elijen en su estudio la longitud de las tuberías), $M_{k}$ índica el módulo al que pertenece la línea $k$, el factor $\delta$ tomará el valor de 1 si $M_{m}$ y $M_{k}$ son iguales y 0 en caso contrario, $W$ es la suma de todos los pesos y $n_{d}$ el número de nuevos elementos a instalar.

Para terminar el proceso, y dado que la solución al problema planteado puede no ser única, los autores plantean usar un algoritmo genético para obtener una serie de soluciones y con ellas construir un frente de Pareto para la ayuda en la toma de decisiones.

Giustolisi et al. (2015) plantean como objetivo una sectorización utilizando un índice de modularidad, pero ajustando este indicador para introducirle, como criterios de similitud entre los nudos, variables hidráulicas como diámetro, presión media o elevación del terreno. Laucelli et al. (2017) buscan organizar todos los nudos de la red en grupos maximizando el índice de modularidad y minimizando el número de líneas que conecten esos grupos. Posteriormente, dan un paso más, y estudian qué tuberías deben cerrar para generar los sectores hidráulicos. Un estudio similar se puede encontrar en Zhang et al. (2017), pero en este caso, además, se busca maximizar el nivel de presiones y controlar los tiempos de permanencia del agua.

Al margen de las técnicas de agrupación, pero dentro de las estructuras comunitarias, cabría destacar la contribución de Herrera et al. (2012) que aplica el agrupamiento espectral para lograr el objetivo planteado. 


\subsubsection{Procesos heurísticos}

Ante un problema que se quiere analizar, la heurística tiene como finalidad el elaborar principios o estrategias como ayuda para hallar la solución más eficaz y eficiente al problema que se pretenda analizar, y sobre el cual es difícil hallar una solución óptima o satisfactoria. Dentro del grupo de procesos heurísticos, y volviendo de nuevo a la técnica de la sectorización, se han englobado todas aquellas metodologías que no se han podido catalogar ni dentro de la Teoría de Grafos ni en las Estructuras Comunitarias.

Las metodologías heurísticas, por regla general, no cambian el enfoque hidráulico clásico de los sistemas de distribución, sino que buscan cómo solucionar los problemas complejos planteados. Herrera et al. (2010) proponen un sistema multi-agente para sectorizar una red en función de los puntos de suministro, minimizando en cada grupo de nudos las diferencias de cota, consumos y energías necesarias para transportar el agua. En este mismo camino, De Paola et al. (2014) proponen buscar el mínimo coste de inversión, el coste asociado a las fugas y el coste energético empleando para ello los algoritmos genéticos. Al igual, pero dándole mayor peso al factor energético, se encuentra la metodología propuesta por Castro et al. (2014). O la metodología expuesta por Di Nardo et. al (2014) donde combina el algoritmo hormiga (para encontrar el camino más corto) con la teoría de grafos (para realizar la partición de la red, y los algoritmos genéticos).

También es importante matizar, que numerosos estudios, que principalmente basan su cálculo o enfoque en otras metodologías, recurren a procesos heurísticos para completar la resolución del problema. Laucelli et al. (2017) buscan organizar todos los nudos de la red en grupos maximizando el índice de modularidad, siguiendo una segmentación compleja, para posteriormente abordar el problema de la ubicación de las válvulas de corte. Un caso similar se encuentra en Pesantez et al. (2019), con la diferencia de seguir un enfoque de teoría de grafos para la definición de los sectores.

\subsection{Evaluación de la sectorización}

Cada vez con más auge, los indicadores de desempeño o de gestión (Alegre et al., 2016; Cabrera Jr. \& Pardo, 2008) se están empleando como herramienta de medida entre los gestores de los sistemas de distribución de agua, y no solo para evaluar el trabajo realizado sino también para comparar diferentes líneas alternativas de actuación (Alegre \& Coelho, 2012). Son muchos y muy variados los indicadores planteados en la actualidad. La IWA (Alegre et al., 2000) presenta unos 170 indicadores agrupados según su naturaleza (operacionales, financieros, calidad del servicio, etc.), o la ERSAR (ERSAR, 2009), con otros 70 indicadores, son solo dos ejemplos.

Dentro del ámbito de la técnica de la sectorización, aunque multitud de los indicadores anteriores tienen cabida, no se han establecido de manera generalizada un grupo en concreto para la evaluación y comparación de diferentes sectorizaciones. En este sentido, han sido pocos los autores que han seleccionado una serie de métricas para la evaluación del comportamiento de la red tras la sectorización (Di Nardo et al., 2014) o para analizar el rendimiento de diferentes planteamientos dentro de una misma red (Di Nardo, 2014; Ferrari \& Savic, 2015). Di Nardo et al. (2013a; 2014) y Tzatchkov et al. (2014) van más allá y plantean la organización de los indicadores en 6 grupos, según su naturaleza, y que son: energía 
disipada, entropía, presión en red, calidad del agua, viabilidad mecánica y capacidad ante incendios.

A continuación, y partiendo de la base de los seis grupos antes mencionados, se desarrollarán las recomendaciones recogidas en la bibliografía referente a los indicadores de gestión enfocados a la técnica de la sectorización.

\subsubsection{Energía disipada en la red}

Una de las principales repercusiones de la sectorización de una red es el cambio en la topología inicial de la misma por el simple hecho del cierre de tuberías para aislar unas zonas de otras. Con ello, el balance de los caudales a lo largo de toda la red se ve modificado, y con ello el balance de energías. Para abordar el comportamiento energético, Di Nardo et al. (2013a; 2014) y Tzatchkov et al. (2014) proponen el empleo de los siguientes indicadores:

- Índice de Fiabilidad (Todini, 2000): Este índice representa la relación entre la potencia que se está disipando a través de la fricción en las tuberías y la máxima energía que se podría disipar (hasta reducir la presión en los nudos a la presión mínima de diseño), según la ecuación 2.8:

$$
I_{r}=1-\frac{P_{i n t}}{P_{\max }}=1-\frac{P_{t o t}-P_{\text {ext }}}{P_{\max }}=\frac{\gamma \sum_{i=1}^{n_{n}} q_{i}\left(h_{i}-h_{i D}\right)}{P_{t o t}-\gamma \sum_{i=1}^{n_{n}} q_{i} \cdot h_{i D}}
$$

Donde $q_{i}$ es el consumo del nudo $i, h_{i}$ corresponde con la altura del nudo, $h_{i D}$ la altura mínima obtenida con la presión mínima de diseño, $P_{\text {tot }}$ es la potencia total entregada al sistema, $n_{n}$ el número de nudos de consumo en el sistema, $P_{\text {int }}$ es la potencia disipada por fricción y la $P_{\text {ext }}$ corresponde con la potencia que se escapa del sistema.

Interpretando este indicador, en el caso ideal, cuando no se pueda disipar más energía por fricción, $I_{r}$ tendrá un valor 0 . En el caso contrario, cuando la energía disponible sea igual a la energía entrante en el sistema, $I_{r}$ será igual a la unidad. En este caso, la fiabilidad del sistema será máxima.

Este mismo índice es también empleado por otros autores. Grayman et al. (2009), por ejemplo, le da a su empleo un enfoque desde la seguridad de la red sectorizada.

- Desviación del Índice de Fiabilidad: Siguiendo el indicador anterior, Di Nardo et al. (2013a) proponen no evaluar la fiabilidad en sí del sistema resultante, sino estudiar la variación de esta tras la sectorización de la red. Emplean para ello la expresión 2.9:

$$
I_{r d}=\left(1-\frac{\mathrm{I}_{\mathrm{r}}^{*}}{\mathrm{I}_{\mathrm{r}}}\right) \cdot 100=\left(\frac{\mathrm{P}_{\text {int }}^{*}-P_{\text {int }}}{\mathrm{P}_{\max }-P_{\text {int }}}\right) \cdot 100
$$

Donde I* corresponde con el índice de Todini pero para la red sectorizada.

- Índice de Resiliencia de la red: Este concepto, introducido por Prasad \& Park (2004), incorpora de manera combinada los efectos de la energía excedente en el sistema y la robustez de las mallas de la red. Se define según la ecuación 2.10:

$$
I_{r n}=\frac{\gamma \sum_{i=1}^{n_{n}} C_{i} \cdot P_{i n t}}{P_{\max }}
$$


Donde $C_{i}$ corresponde con el cálculo de la uniformidad de cada nudo según la siguiente ecuación:

$$
C_{i}=\frac{\sum_{j=1}^{M_{i}} d_{j}}{M_{i} \cdot \max \left\{d_{1} \cdots d_{M_{i}}\right\}}
$$

Considerando $M_{i}$ el número de líneas incidentes en el nudo i y $d_{j}$ el diámetro de la línea j.

El índice de Resiliencia está fuertemente relacionado con la capacidad intrínseca del sistema para superar las fallas y al mismo tiempo satisfacer las demandas y presiones de los nudos (Baños et al., 2011). Su valor está comprendido en el rango continuo entre 0 y 1 , donde altos valores significan altas cantidades de energía excedente y una distribución más uniforme de tuberías de distribución y, por lo tanto, una mayor robustez de la red.

- Desviación del Índice de Resiliencia: Al igual que con el índice de fiabilidad, Di Nardo et al. (2013a) proponen estudiar la variación del indicador anterior para comprobar el efecto de la sectorización sobre la red, según la ecuación 2.12:

$$
I_{r n d}=\left(1-\frac{I_{r n}^{*}}{I_{r n}}\right) \cdot 100
$$

Donde $I_{r n}^{*}$ es el índice de robustez de la red sectorizada.

Respecto a la interpretación de este indicador, valores altos corresponden con peores comportamientos de la red sectorizada.

Llegados a este punto, es importante destacar que los anteriores indicadores han sido agrupados por los autores citados bajo el grupo de energía disipada en la red. Pero cierto es que pueden emplearse para evaluar otros criterios, como lo recogido en Herrera et al. (2016), donde se estudia la capacidad de un sistema para mantener su nivel de rendimiento bajo alteraciones de su estado normal empleando para ello los índices de Fiablidad y Resiliencia, entre otros.

\subsubsection{Entropía}

Desde un punto de suministro hasta un punto de consumo, el flujo tendrá diferentes caminos a recorrer según la tipología que presente la red. Y según sea esta, la red responderá en mayor o menor grado ante cualquier tipo de contingencia. Para medir la uniformidad del flujo en las tuberías Prasad \& Tanyimboh (2008), Di Nardo et al. (2013a; 2014) y Tzatchkov et al. (2014) proponen abordar el concepto de entropía formulado por Shannon (1948), según el cual, y bajo la óptica matemática, la entropía aplicada a las redes de distribución se podría entender como todos los caminos que tiene el flujo para llevar agua a un punto de consumo desde el punto de suministro y la probabilidad de que el agua fluya por ellos. Para estudiar estos caminos, los autores proponen usar la expresión 2.13, planteada por Tanyimboh \& Templeman (1993) para su uso en los sistemas de distribución de agua: 


$$
S=\sum_{i=1}^{N_{d}} S_{i}=\sum_{i=1}^{N_{d}} \sum_{j=1}^{N_{i}}\left[-P_{i j} \ln P_{i j}+P_{i j} S_{j}\right]
$$

Donde $S_{i}$ y $S_{j}$ es la entropía de los nudos $i$ y $j$ respectivamente, $N_{d}$ corresponde con los nudos con demanda no nula, $N_{i}$ los nudos desde los cuales el flujo alcanza al $i$-ésimo nudo y $P_{i j}$ es la probabilidad de que el agua fluya a través del nudo $i$ hasta el $j$, y se expresa como la relación entre el caudal entre $i$-ésimo nudo y el $j$-ésimo nudo y el caudal total que llega al nudo $i$, según la siguiente expresión:

$$
P_{i j}={ }^{q_{i j}} / Q_{i}^{t o t}
$$

En esta línea, Scarpa et al. (2016) proponen el empleo del índice de entropía introducido por Awumah et al. (1991) y que se recoge en la expresión 2.15:

$$
S_{\text {tot }}=\sum_{j=1}^{n}\left(\sum_{i=1}^{n(j)} \frac{q_{i j}}{Q_{0}} \cdot \ln \frac{q_{i j}}{Q_{0}}\right)
$$

Donde $n$ es el número total de nudos, $q_{i j}$ el flujo de la $i$-ésima tubería de las $n(j)$ tuberías que llegan al nudo $j$ y $Q_{0}$ es la suma de los flujos de todas las tuberías.

Nótese que el concepto de entropía, adaptado por cada autor según sus necesidades, está muy relacionado con la redundancia mecánica y el grado de mallado de la red.

\subsubsection{Nivel de presión en la red}

El nivel de presiones se ha usado tradicionalmente para evaluar el nivel de servicio ofrecido en los sistemas de distribución de agua. Principalmente, se ha recurrido a los niveles medios, mínimos, máximos y la desviación de estos. Y en el caso de la sectorización de las redes, los diseñadores recurren a este parámetro para evaluar, de una manera rápida, la efectividad de esta, desde la perspectiva de la calidad en el suministro. De manera complementaria, Di Nardo et al. (2013a; 2014) y Tzatchkov et al. (2014) proponen los siguientes indicadores basados en los niveles de presión en los nudos:

- Exceso de presión media: La cual se calcula como:

$$
E P M=\frac{\sum_{i=1}^{n_{n}} \alpha_{i} \cdot q_{i}}{\sum_{i=1}^{n_{n}} q_{i}} \quad \operatorname{con} \begin{cases}\alpha_{i}=0 & \text { si } p_{i} \leq p_{i_{D}} \\ \alpha_{i}=p_{i}-p_{i_{D}} & \text { si } p_{i}>p_{i_{D}}\end{cases}
$$

Donde $n_{n}$ corresponde con el número de nudos, $q_{i}$ es la demanda del nudo $i, p_{i}$ es la presión en el nudo $i$ y $p_{i_{D}}$ es la presión de diseño en el nudo $i$. Un valor alto de este indicador corresponde con un exceso en la presión de la red.

- Déficit medio de presión: En línea con el anterior indicador, se propone comparar o medir el descenso de la presión comparado con la presión de diseño, según la siguiente ecuación:

$$
D M P=\frac{\sum_{i=1}^{n_{n}} \beta_{i} \cdot q_{i}}{\sum_{i=1}^{n_{n}} q_{i}} \quad \text { con } \begin{cases}\beta_{i}=0 & \text { si } p_{i} \geq p_{i_{D}} \\ \beta_{i}=p_{i}-p_{i_{D}} & \text { si } p_{i}<p_{i_{D}}\end{cases}
$$


En este caso, altos valores del déficit de presión conllevarán peores condiciones de servicio.

- Desviación de la presión respecto a la presión de diseño: Este último indicador trata de obtener la desviación media de la presión en los nudos respecto a la presión de diseño. Se calcula según la expresión 2.18:

$$
M S P D=\sqrt{\frac{\sum_{i=1}^{n_{n}}\left(p_{i}-p_{i_{D}}\right)^{2}}{n_{n}}}
$$

Analizando este parámetro, valores altos significan mayores diferencias entre los niveles de presiones en los nudos y el nivel de diseño. Al contrario, niveles bajos implican niveles de presiones más ajustados al valor de diseño.

Nuevamente, y siguiendo esta línea, Scarpa et al. (2016) proponen, para analizar las redes sectorizadas, el empleo del siguiente indicador introducido por Coelho (1997) (HP - Hydraulic performance pressure related):

$$
H P=\sum_{j=1}^{n_{n}} \frac{q_{j}}{q_{0}} \cdot i_{p_{j}}
$$

Donde $q_{j}$ es la demanda del nudo $j, q_{0}$ es la demanda total de la red y el término $i_{p_{j}}$ es un peso asociado al nivel de presión en dicho nudo, siendo un valor de 3 para la presión adecuada de servicio, 2 para un nivel aceptable de servicio $(>1,25 \cdot$ presión de servicio), 1 para una presión inaceptable $(>1,75 \cdot$ presión de servicio) y 0 cuando no se consigue suministrar con la presión mínima de servicio $(<0,75 \cdot$ presión de servicio). Así, una red con un valor HP alto significa que las presiones en los nudos están cerca del nivel de servicio, siendo mínimo el desperdicio de energía a la hora de satisfacer las demandas.

\subsubsection{Calidad del agua}

Los dos principales mecanísmos de deterioro de la calidad del agua son las interacciones entre la pared de la tubería y esta y las reacciones dentro de su propia masa (EPA, 2002). En ambos casos, la edad del agua es un factor importante que contribuye al deterioro de la calidad de la misma dentro del sistema de distribución. Y a su vez, el tiempo que el agua permanece dentro del sistema depende directamente de la demada y de la topología y operación del sistema.

Por ello, y para evaluar el efecto que produce la implantación de una sectorización sobre la calidad del agua, gran parte de los autores (Di Nardo et al., 2013a; 2014; Tzatchkov et al., 2014; Grayman et al., 2009) proponen el uso del tiempo de permanencia del agua en la red. Emplean para ello el uso del software Epanet (Rossman, 2002) para obtener los valores máximos, medios y las desviaciones sufridas de los escenarios planteados.

Debido a que la calidad del agua depende de muchos factores (caudal circulante, materiales empleados, calidad inicial del agua, etc) es complejo establecer unas recomendaciones globales para todos los sistemas. Tanto es así que la literatura recoge ejemplos que van desde horas hasta días (EPA, 2002). En el caso de la American Water Works Associtation recomienda un tiempo de permanencia medio de 1,3 días y un máximo de 3 (AWWA \& AWWARF, 1992). 
Armand et al. (2015) profundizaron más sobre en esta materia y presentan un análisis de quejas recibidas por los abonados durante un periodo de tiempo considerable, en las cuales analizan los efectos de la sedimentación, turbidez, acumulaciones o el bio-film de las tuberías.

\subsubsection{Resiliencia mecánica}

En términos generales, la habilidad o capacidad de un sistema de distribución de agua para satisfacer las demandas del consumidor en condiciones normales o no, pero siempre en cantidad y calidad, se considera confiabilidad de la red (Farmani et al, 2005; Herrera et al., 2016b; Cheng et al., 2020). Y aunque esta capacidad de los sistemas ha sido ampliamente abordada en la bibliografía (Goulter et al. 2000), el concepto de este fenómeno es muy abstracto y su análisis complicado debido a que la capacidad de respuesta depende de las condiciones de contorno establecidas (Monsef et al., 2019).

Tradicionalmente, se ha usado el concepto de confiablidad para evaluar y diseñar los sistemas desde el punto de vista de la seguridad (Cheng et al., 2020). Principalmente, evaluando dos cuestiones igual de importantes: el nivel de agua suministrada y el grado de importancia de cada tubería de manera local o global en el sistema. De manera complementaria a lo apuntado por el autor anterior, Yazdani \& Jeffrey (2011) destacan la importancia de evaluar la forma, estructura, eficiencia y vulnerabilidad de las redes de distribución desde el punto de vista de su estructura y su seguridad de funcionamiento y la susceptibilidad a los daños.

Así, existen diferentes indicadores para medir esta capacidad del sistema. Gheisi et al. (2016) realizan una revisión de todos ellos, clasificándolos en dos grupos principales: la fiabilidad hidráulica, que se refiere a la tolerancia de la red ante los cambios de la demanda (Ostfeld et al., 2002), y la fiabilidad mecánica, que se refiere a la tolerancia frente a los fallos de las infraestructuras (Atkinson et al., 2014). Se han definido muchos parámetros para medir la fiabilidad hidráulica, tales como la altura mínima de suministro, el índice de fiabilidad y el índice de resiliencia. En el caso de la medición de la fiabilidad mecánica, un parámetro para ello puede ser el indicador de fiabilidad de entropía (Tanyimboh \& Templeman, 2000).

\subsubsection{Capacidad de la red ante condiciones de incendio}

La respuesta hidráulica de las redes de distribución, o más bien la capacidad de respuesta, ante situaciones de incendio queda englobada bajo el paraguas de la resiliencia de estas. No es más que la capacidad de reacción ante un escenario de contingencia, aunque con la salvedad de que es un requisito legalmente exigido en un gran número de sistemas. Y de ahí la necesidad de estudiarlo por separado.

Tradicionalmente, este análisis se ha soportado en el uso del modelo de simulación y en el estudio de los escenarios más desfavorables. En el caso de España, es necesario cumplir con las exigencias impuestas por el Código Técnico de la Edificación (RD 314/2006, de 17 de marzo) el cual establece, entre otros requisitos, que ante situaciones de incendios las redes de distribución deben garantizar un caudal mínimo de 1.000 litros por minuto en dos hidrantes consecutivos y una presión mínima de 10 mca. En el caso de México (Tzatchkov, 2007), se requiere una presión mínima en la toma de 5 mca y el caudal garantizado depende del número de habitantes de la localidad, no siendo un requisito en poblaciones pequeñas. Por otro lado, en Inglaterra y para gran parte de las utilities sí se obliga a la instalación de equipos para la lucha contra incendios, pero no impone el diseño de los sistemas de distribución bajo las demandas pico que ello requiere (United Utilities Water plc, 2015). 
Para estudiar el comportamiento de la red bajo condiciones de incendio, Di Nardo et al. (2013a; 2014) y Tzatchkov et al. (2014) proponen el uso del Índice de protección contra incendios (IPI), que queda definido en la ecuación 2.20, y tiene como objetivo el poder comparar diferentes escenarios según el número de nudos con presión inferior a la presión requerida en condiciones de incendio:

$$
I P I=\sum_{i=1}^{n} x_{i} \quad \text { en el cual }\left\{\begin{array}{ll}
x_{i}=0 & \forall i: p_{i} \geq p_{P I} \\
x_{i}=1 & \forall i: p_{i}<p_{P I}
\end{array}\right\}
$$

Donde $n$ es el número de nudos, $p_{i}$ es la presión en el nudo $i$ en condiciones de incendio y $p_{P I}$ es la presión mínima requerida en dichas condiciones. 


\section{Capítulo 3 \\ Aplicación de conceptos energéticos en la fase de diseño}

\subsection{Introducción}

La interdependencia entre el agua y la energía es más que clara. Se requiere energía para poder mover el agua y es preciso el uso de agua para producir gran parte de la energía que consume la actividad y desarrollo humano. Esta relación ha sido ampliamente abordada por la comunidad científica, a la cual se refieren como el nexo agua-energía.

Los enfoques más directos y específicos se centran en cuantificar ambos recursos. Hardy et al. (2012) analizan, para el caso de España, qué cantidad de energía $\left(\mathrm{kWh} / \mathrm{m}^{3}\right)$ es usada en el sector del agua y la necesidad de agua por unidad de energía producida ( $\mathrm{m}^{3} / \mathrm{GWh}$ ). De dicho estudio, se puede destacar que el 5,8\% del consumo energético español es debido al sector del agua, donde la extracción y el tratamiento se llevan el 64\%. Fuera de España, se encuentran valores del 2-3\%, en el caso de Inglaterra, del 13\% para Estados Unidos (Vakilifard et al., 2017) o un valor medio mundial del 7-8\% (Sharif et al., 2019). Otros autores, como Fang \& Chen (2017), abordan la relación entre el nexo agua-energía y la estructura económica de un sistema urbano, todo ello con el objetivo de lograr una gestión de los recursos más eficiente. El mismo enfoque, pero a nivel global, es el seguido por Biggs et al. (2015). Siguiendo con la perspectiva global, recientemente Lee et al. (2017) aborda las consecuencias e impactos del uso del agua y la energía sobre el estrés hídrico de determinadas zonas.

Por todo ello, se hace notorio que las políticas sobre la gestión del agua deberán contemplar las restricciones impuestas por la energía, y viceversa (Scott et al., 2011). Claros ejemplo de ello se encuentran en Australia, donde el consumo energético de muchas ciudades se verá aumentado notablemente si se implementan fuentes alternativas de agua basadas en la desalinización o el reciclaje de aguas residuales (Lee et al., 2017). O en países como 
Sudáfrica, Marruecos, México o China, donde están trabajando en incorporar las restricciones impuestas por el agua en sus plantas de producción de energía (Lee et al., 2017).

Centrados en los sistemas de distribución de agua, la energía, de una manera u otra, está presente en todas las fases. En la fase de diseño, son numerosos los estudios que lo abordan considerando el criterio energético como una de las variables de decisión (Babayan et al., 2005; Dandy et al., 2008; Zheng et al., 2011; Lence et al., 2017), y todos ellos con un objetivo en común: la reducción del consumo energético y de operación. En la fase de operación y control, la energía supone un gran porcentaje dentro de los costes de operación de las infraestructuras. Este peso dependerá, en gran medida, de la tipología y la eficiencia de la infraestructura, pero puede variar desde valores mínimos del 2\% (Ortí et al., 2008), valores medios del 11\% (Ministerio de Medio Ambiente, 2007) o incluso suponer hasta el $58 \%$ de los ingresos totales (Consejería de Agricultura, Pesca y Medio Ambiente, 2009). El número de estudios que abordan el control y operación de los sistemas con el objetivo de la optimización energética son cuantiosos (López \& Martinez, 2006; Burn et al., 2007; Coelho \& AndradeCampos, 2014; Gómez, 2016). Por detallar alguno de ellos, y dentro del ámbito de la línea de investigación seguida, se destaca el trabajo de Nicolás et al. (2014), en el cual se propone un estudio basado en sectorizar la red y usar variadores de velocidad como medida de eficiencia energética, para minimizar los costes totales.

Queda claro con todo ello que, la energía, más concretamente el consumo energético, tiene una especial relevancia en los sistemas de distribución de agua. Pero en este capítulo, se dejará al margen el concepto tradicional (que finalmente se traduce en un coste económico) y se recurrirá al concepto teórico del mismo. Con este enfoque, se plantean nuevos criterios para la definición de sectores hidráulicos, cuya definición ayude a una óptima gestión de la presión posterior.

\section{$3.2 \mathrm{El}$ concepto energético en los sistemas de distribución}

Tras la fase de diseño y dentro del control y operación, es importante gestionar el uso de la energía. Y para ello, es necesario conocer dónde y cómo se está empleando. Con este propósito, Pelli \& Hitz (2000) presentan dos indicadores para evaluar cómo es el consumo de energía en pequeños o medianos sistemas de distribución. El primero de ellos, Structure Indicator, relaciona la mínima energía requerida para el suministro y el volumen total suministrado y es un indicador de la distribución espacial de los puntos de suministro y los consumidores. Se trata de un indicador sencillo de calcular, pero limitado, ya que ignora el efecto de la fricción en las tuberías. El segundo indicador propuesto, Quality Indicator, evalúa, en cierto modo, la eficiencia del sistema y trata de relacionar la energía consumida en el sistema con la mínima requerida. En la misma línea, Colombo \& Karney (2002) abordan la relación entre el consumo energético y las fugas, calculando cómo varía el primero según sea el tipo de fuga y la ubicación de ésta. Filion et al. (2004) presentan un estudio que analiza la energía que interviene en cada una de las etapas del ciclo de vida de las tuberías: analizan la energía requerida para su fabricación, la que se pierde por fricción o la requerida para reparar las roturas ocasionadas en dichas tuberías y, finalmente, la energía necesaria para su desecho o reciclaje. 
Boulos \& Bros (2010) introducen una simple, pero completa, metodología para evaluar la eficiencia energética de toda la red de distribución, desde el punto de entrada al sistema hasta el punto de entrega al consumidor. Este indicador lo definen como la relación entre la potencia requerida para alcanzar la presión mínima frente a la actual potencia empleada:

$$
\eta_{e}=\frac{P_{\min }}{P_{t}}
$$

Siendo $P_{t}$ la potencia consumida y $P_{\min }$ la potencia mínima necesaria para mantener el mínimo nivel de presión requerido en el punto de consumo, y que se calcula según la siguiente expresión:

$$
P_{\text {min }}=\rho \cdot g \cdot \sum_{k=1}^{n} q_{k} \cdot H_{\text {min }}
$$

Donde $n$ es el número de nudos, $q_{k}$ la demanda en el nudo $k$ y $H_{\min }$ la mínima altura piezométrica admisible en el nudo $k$.

Uno de los objetivos que Boulos y Bross intentan conseguir con la definición de esta nueva medida de la eficiencia energética de las redes de distribución es el dar a conocer aquellas zonas o redes de distribución con menores rendimientos y por lo tanto con mayores potenciales de ahorro energético.

Con un enfoque más detallado, y de forma similar a los balances y auditorías hídricas realizadas sobre los sistemas de distribución, Cabrera et al. (2010) presentan un balance energético de aplicación en las redes de distribución de agua. Esta auditoría energética tiene presente todos los flujos de energía entrantes, salientes o almacenadas que permiten la

\begin{tabular}{|c|c|c|c|}
\hline \multirow{3}{*}{$E_{\text {INPUT }}$} & \multirow{2}{*}{$\begin{array}{c}\mathrm{E}_{\mathrm{N}} \\
\text { Energía natural } \\
\text { suministrada }\end{array}$} & $\begin{array}{c}\text { EU } \\
\text { Energía útil cedida con la } \\
\text { demanda de caudal de los } \\
\text { usuarios }\end{array}$ & \multirow{2}{*}{$E_{\text {output }}$} \\
\hline & & $\begin{array}{c}\mathrm{E}_{\mathrm{L}} \\
\underset{\text { Energía asociada a las }}{\text { fugas }}\end{array}$ & \\
\hline & $\begin{array}{c}E_{P} \\
\begin{array}{c}\text { Energía de eje (grupos de } \\
\text { presión) }\end{array}\end{array}$ & $\begin{array}{c}\mathrm{E}_{\mathrm{F}} \\
\text { Energía invertida en } \\
\text { vencer el rozamiento }\end{array}$ & $E_{\text {Disipada }}$ \\
\hline
\end{tabular}
distribución del agua.

Figura 3.1. Balance energético propuesto por Cabrera et al. (2010)

Para un sistema con $n$ nudos de consumo y $n_{l}$ tuberías, alimentados desde $n_{N}$ embalses, con $n_{P}$ bombeos que aportan energía al sistema y con $n_{C}$ depósitos de compensación, los resultados de la auditoría energética planteada se recogen en la siguiente ecuación: 


$$
\begin{aligned}
\gamma \sum_{i=1}^{i=n_{N}} & \left(\sum_{t_{k}=t_{1}}^{t_{k}=t_{p}} Q_{N i}\left(t_{k}\right) \cdot H_{N i}\left(t_{k}\right) \cdot \Delta t_{k}\right)+\gamma \sum_{i=1}^{i=n_{P}}\left(\sum_{t_{k}=t_{1}}^{t_{k}=t_{p}} Q_{P i}\left(t_{k}\right) \cdot H_{P i}\left(t_{k}\right)\right) \Delta t= \\
= & \gamma \sum_{i=1}^{i=n}\left(\sum_{t_{k}=t_{1}}^{t_{k}=t_{p}} q_{u i}\left(t_{k}\right) \cdot H_{i}\left(t_{k}\right)\right) \cdot \Delta t+\gamma \cdot \sum_{i=1}^{i=n}\left(\sum_{t_{k}=t_{1}}^{t_{k}=t_{p}} q_{l i}\left(t_{k}\right) \cdot H_{i}\left(t_{k}\right)\right) \cdot \Delta t \\
& +\gamma \sum_{i=1}^{i=n_{L}}\left(\sum_{t_{k}=t_{1}}^{t_{k}=t_{p}}\left(q_{u j}\left(t_{k}\right)+q_{l j}\left(t_{k}\right)\right) \cdot \Delta h_{j}\left(t_{k}\right)\right) \cdot \Delta t+\sum_{i=1}^{n_{C}} \frac{\gamma \cdot A_{i}}{2}\left(z_{i}^{2}\left(t_{f}\right)-z_{i}^{2}\left(t_{i}\right)\right)
\end{aligned}
$$

donde $\gamma$ es el peso específico del agua, $Q_{N i}\left(t_{k}\right)$ es el caudal aportado por el embalse en el instante $t_{k}, H_{N i}\left(t_{k}\right)$ es su altura piezométrica, $\Delta t_{k}$ el intervalo de tiempo considerado, $Q_{P i}\left(t_{k}\right)$ es el caudal trasegado por el equipo de bombeo, $H_{P}\left(t_{k}\right)$ es su altura de bombeo en ese mismo instante, $q_{u i}\left(t_{k}\right)$ es el caudal entregado a los abonados en el nudo $i, H_{i}\left(t_{k}\right)$ es la altura piezométrica en el nudo $i, q_{l i}\left(t_{k}\right)$ es el caudal fugado en las tuberías adyacentes al nudo $i$ en el instante $t_{k}, \Delta h_{j}\left(t_{k}\right)$ la pérdida de carga de la línea $j, q_{u j}\left(t_{k}\right)$ es el caudal de la línea $j$ necesario para atender la demanda de los usuario, $q_{l j}\left(t_{k}\right)$ es el caudal de la línea $j$ que finalmente se perderá por fugas, $A_{i}$ es la sección del i-ésimo depósito, mientras $z_{i}\left(t_{f}\right)$ y $z_{i}\left(t_{i}\right)$ son los niveles de la superficie libre de agua en el depósito i en los instantes de tiempo extremos.

O de una manera mucho más compacta:

$$
E_{\text {input }}=E_{N}\left(t_{P}\right)+E_{P}\left(t_{P}\right)=E_{U}\left(t_{P}\right)+E_{L}\left(t_{P}\right)+E_{F}\left(t_{P}\right) \pm \Delta E_{C}\left(t_{P}\right)
$$

El trabajo no plantea únicamente la anterior auditoría energética, sino que propone una serie de indicadores energéticos para la evaluación de las redes de distribución, desde una visión energética de la misma. Así, los autores definen un total de ocho indicadores energéticos: dos indicadores de contexto, que dependen únicamente de las características físicas de la red, y seis indicadores energéticos, los cuales permiten determinar cuán eficiente es la gestión de la red.

De todos ellos y en línea con la investigación seguida, es interesante destacar dos. El primero es el indicador de contexto $\mathrm{C}_{2}$, Exigencia energética de la red. Este indicador es el cociente entre la energía mínima útil necesario, definida en cada nudo a partir de la altura mínima, y la energía mínima teórica requerida por una red plana e ideal (sin fugas y sin fricción). Su cálculo es el siguiente:

$$
C_{2}=\frac{E_{\text {min,useful }}}{E_{\text {min, flat }}}=\frac{\gamma \cdot \sum_{t_{k}=t_{1}}^{t_{k}=t_{p}}\left(\sum_{i=n}^{i=n} q_{u i}\left(t_{k}\right) \cdot\left(h_{\text {min }}\right)_{i}\right) \cdot \Delta t}{\gamma \cdot \frac{P_{\text {min }}}{\gamma} \cdot V_{U}\left(t_{P}\right)}
$$

El segundo es el indicador energético $\mathrm{I}_{5}$, Suficiencia en el cumplimiento de estándares. Este indicador es directamente el cociente entre la energía entregada a los usuarios y la energía mínima útil necesaria:

$$
I_{5}=\frac{E_{U}\left(t_{P}\right)}{\gamma \cdot \sum_{i=1}^{n} v_{u, i}\left(t_{P}\right) \cdot h_{\min , i}}
$$


Nótese que este último indicador es similar a la eficiencia energética propuesta por Boulos y Bross, aunque en este caso el cálculo se realiza con energías y no con potencias.

Bolognesi et al. (2014) defienden que es tan importante el conocer cuánta energía se consume, cómo el conocer la eficiencia con la que se realiza dicho consumo. Para ello, estos autores proponen el concepto de energía mínima inevitable como referencia para definir un indicador de eficiencia energética. Aunque este concepto va en la línea de lo expuesto por los dos trabajos anteriores, estos autores tan solo enfocan este concepto de eficiencia sobre equipos de bombeo.

En un estudio posterior, Cabrera et al. (2015) desglosa la energía entregada a los usuarios en tres conceptos:

$$
E_{U}=E_{u o}+E_{t r}+E_{\text {er }}
$$

donde $E_{u o}$ es la energía mínima requerida que debe suministrarse a los usuarios para cumplir con la presión mínima establecida y el caudal demandado; $E_{t r}$ corresponde con la energía topográfica y representa la energía que se debe aportar para alcanzar la presión mínima en la cota del nudo crítico y $E_{\text {er }}$ es la energía de exceso sobre la energía mínima requerida en el nudo crítico y en el resto de los nudos. Gracias a este desglose, y al margen de los indicadores planteados en el anterior estudio, definen el Rendimiento real como un indicador energético que tiene presente las pérdidas energéticas y permite cuantificar la eficiencia de la red relacionando la energía mínima necesaria para suministrar la demanda requerida por los usuarios $\left(E_{u o}\right)$ y la energía real suministrada al sistema $\left(E_{s r}\right)$. Así, el rendimiento real se calcula según la siguiente expresión:

$$
\eta_{a r}=\frac{E_{u o}}{E_{s r}}
$$

En último lugar, Smith et al. (2016) analiza la cantidad de gases de efecto invernadero emitidas a la atmósfera debido a la energía usada en los sistemas de distribución de agua, centrando el estudio en China y en la relación directa entre el volumen incontrolado fugado y la cantidad de gases emitidos.

\subsection{Diseño de sectorizaciones basado en conceptos energéticos}

En el apartado anterior, se han abordado conceptos energéticos aplicados a los sistemas de distribución de agua. En concreto, en la fase de gestión son varios los estudios que plantean el análisis energético con el objetivo de evaluar aquellos sistemas con mayor margen de mejora. Este es el caso de la eficiencia energética, presentada por Boulos \& Bross (2010), o la Suficiencia en el cumplimiento de estándares, presentada por Cabrera et al. (2010). Ambos indicadores buscan la relación entre los niveles actuales de energía y los mínimos necesarios para cumplir con el servicio. A lo largo de este apartado, se seguirá en la línea de los citados indicadores para la definición de los sectores hidráulicos. 
Como ya se abordó en el segundo capítulo, la sectorización de redes consiste en agrupar los elementos en grupos con cierto grado de independencia entre sí. Las ventajas que se consiguen eran más que evidentes para la gestión de las redes, desde diferentes puntos de vista e independientemente de la distribución de los sectores. Ahora bien, esta distribución influirá en el punto de funcionamiento de la red. Y de cara a una posterior optimización de este funcionamiento, como por ejemplo una política de gestión de presiones, los resultados obtenidos serán diferentes en función de dicha dispersión de los sectores. Por ello, se puede afirmar que ciertas sectorizaciones permitirán mayores logros que otras en posteriores procesos de optimización. O, dicho de otro modo, para una misma red, unas distribuciones sectoriales ayudarán más que otras en la gestión global de las mismas.

\subsubsection{La sectorización desde la óptica monocriterio (energética)}

Para profundizar en dicho aspecto, véase el perfil de la red mostrada en la Figura 3.2. Se trata de una red ramificada y sencilla. La red está compuesta por un depósito de cabecera que alimenta a cinco nudos de consumo, cuyas características se muestran en la Tabla 3.1. Para una mayor compresión de los resultados, se asumirá que el sistema no presenta pérdidas por fricción.

Se quiere sectorizar dicha red. Para ello se plantean dos escenarios: Un primer escenario que englobe los nudos 1, 2 y 3 en el primer sector, quedando los nudos 4 y 5 en el segundo sector, y un segundo escenario con un sector englobando los nudos 1 y 2 y el resto en el segundo. Posteriormente, se implantará una gestión de presiones para todo el sistema. De igual manera, se plantean cuatro alternativas de acción: no realizar una gestión de presión, actuar sobre el primer sector, sobre el segundo o de manera conjunta en la entrada de ambos sectores. Así, se tendrán un total de 8 combinaciones posibles a analizar, 4 para cada escenario.

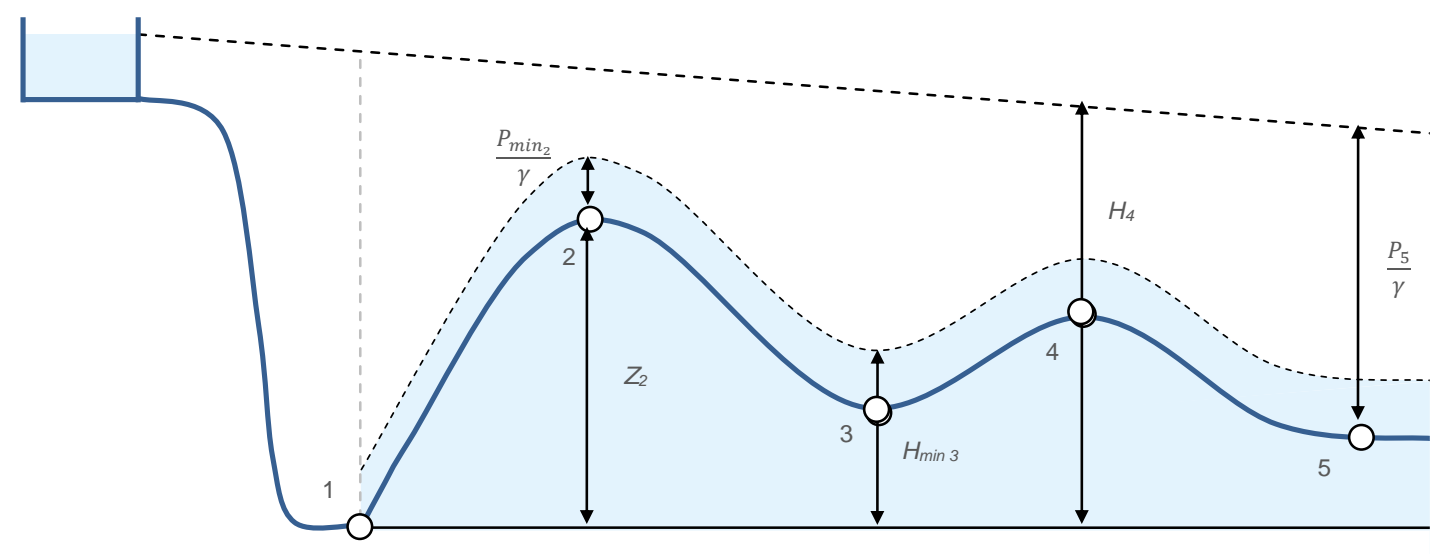

Figura 3.2. Red de distribución por gravedad (vista en perfil)

Tabla 3.1 Características de los nudos de consumo de la Figura 3.2

\begin{tabular}{|c|c|c|c|c|c|c|c|c|c|c|}
\hline & $\underset{(\mathrm{m})}{\mathrm{z}}$ & $\begin{array}{c}P \\
\text { (mca) }\end{array}$ & $\begin{array}{c}\mathrm{H} \\
\text { (mca) }\end{array}$ & $\begin{array}{l}P \text { min } \\
\text { (mca) }\end{array}$ & $\begin{array}{l}\text { Demanda } \\
\quad \text { (Ips) }\end{array}$ & $\begin{array}{c}\text { Q fugas } \\
\text { (Ips) }\end{array}$ & $\begin{array}{l}\text { Energía } \\
\text { útil (kWh) }\end{array}$ & $\begin{array}{c}\text { Energía } \\
\text { fugas } \\
\text { (kWh) }\end{array}$ & $\begin{array}{c}\text { Energía } \\
\text { mínima } \\
\text { (kWh) }\end{array}$ & $\begin{array}{l}\text { Eficiencia } \\
\text { energética }\end{array}$ \\
\hline Nudo 1 & 0 & 70 & 70 & 15 & 1 & 0,4 & 0,69 & 0,27 & 0,15 & \multirow{5}{*}{$46 \%$} \\
\hline Nudo 2 & 35 & 33 & 68 & 15 & 2 & 0,3 & 1,33 & 0,20 & 0,98 & \\
\hline Nudo 3 & 12 & 53 & 65 & 15 & 5 & 0,2 & 3,19 & 0,13 & 1,32 & \\
\hline Nudo 4 & 23 & 40 & 63 & 15 & 6 & 0,2 & 3,71 & 0,12 & 2,24 & \\
\hline Nudo 5 & 10 & 50 & 60 & 15 & 8 & 0,2 & 4,71 & 0,12 & 1,96 & \\
\hline
\end{tabular}


De cara a analizar los diferentes resultados, en primer lugar, se abordará el primer escenario, en el cual se engloban los nudos 1, 2 y 3 en el primer sector, quedando los nudos 4 y 5 en el segundo sector. Según esta distribución (Tabla 3.2), el sector 1 presenta una peor eficiencia energética. Este resultado desprende un mayor distanciamiento entre el nivel de energía disponible en el punto de consumo y el mínimo necesario, y por ende un mayor margen de mejora desde el punto de vista de la eficiencia. Ahora bien, entrando en términos absolutos de energía, es el sector 2 el que presenta un exceso de energía mayor.

Tabla 3.2 Escenario 1, alternativa presión 1.1 - Características energéticas de los sectores 1 y 2

\begin{tabular}{|c|c|c|c|c|c|c|c|c|c|}
\hline Sector & Nudos & $\underset{\text { (mca) }}{\mathrm{H}}$ & $\underset{\text { (mca) }}{\mathbf{P}}$ & $\begin{array}{c}\text { Energía } \\
\text { útil } \\
\text { (kWh) }\end{array}$ & $\begin{array}{c}\text { Energía } \\
\text { fugas } \\
\text { (kWh) }\end{array}$ & $\begin{array}{c}\text { Energía } \\
\text { mínima } \\
\text { (kWh) }\end{array}$ & $\begin{array}{c}\text { Eficiencia } \\
\text { energética }\end{array}$ & $\begin{array}{c}\text { Exceso } \\
\text { Energía } \\
\text { (kWh) }\end{array}$ & $\begin{array}{c}\text { Ahorro } \\
\text { máximo } \\
\text { cons. } \\
\text { energético }\end{array}$ \\
\hline \multirow{3}{*}{ Sector 1} & 1 & 70 & 70 & \multirow{3}{*}{5,2} & \multirow{3}{*}{0,6} & \multirow{3}{*}{2,7} & \multirow{3}{*}{$47 \%$} & \multirow{3}{*}{3,1} & \multirow{3}{*}{$42 \%$} \\
\hline & 2 & 68 & 33 & & & & & & \\
\hline & 3 & 65 & 53 & & & & & & \\
\hline \multirow{2}{*}{ Sector 2} & 4 & 63 & 40 & \multirow{2}{*}{8,4} & \multirow{2}{*}{0,2} & \multirow{2}{*}{4,3} & \multirow{2}{*}{$50 \%$} & \multirow{2}{*}{4,3} & \multirow{2}{*}{$58 \%$} \\
\hline & 5 & 60 & 50 & & & & & & \\
\hline
\end{tabular}

Siguiendo con esta distribución de sectores, se procederá a implementar una gestión de presiones en ambos sectores. Se comenzará (Tabla 3.3) implantando una reducción de presión en el sector 1 , con una única válvula reductora a la entrada del sector, de tal manera que se alcance la presión mínima admisible en el punto crítico de este sector. Así, el nudo crítico sería el nudo 2 y se podría reducir su presión hasta los $15 \mathrm{mca}$, viéndose afectados el resto de los nudos de la red. Acto seguido, y de igual manera, se analizará la alternativa de actuación para la gestión de presión 1.3, reduciendo la presión únicamente en el sector 2 (0). En este caso, el nudo crítico es el 4 y su presión mínima quedará en 15 mca. En esta alternativa, solo se verán afectados los nudos del sector 2. Y finalmente, se analizará la última alternativa, con una reducción de presión máxima admisible en toda la red (Tabla 3.5) empleando sendas válvulas reductoras en cada entrada sectorial. Actuando sobre las dos entradas, es posible conseguir los mínimos niveles de presión en ambos sectores, en los nudos 2 y 4 .

Tabla 3.3 Escenario 1, alternativa presión 1.2 - Gestión de presión en el sector 1

\begin{tabular}{|c|c|c|c|c|c|c|c|c|c|}
\hline Sector & Nudos & $\underset{\text { (mca) }}{\mathrm{H}}$ & $\underset{\text { (mca) }}{P}$ & $\begin{array}{c}\text { Energía } \\
\text { útil } \\
\text { (kWh) }\end{array}$ & $\begin{array}{l}\text { Energía } \\
\text { fugas } \\
\text { (kWh) }\end{array}$ & $\begin{array}{l}\text { Energía } \\
\text { mínima } \\
(\text { kWh) }\end{array}$ & $\begin{array}{l}\text { Eficiencia } \\
\text { energética }\end{array}$ & $\begin{array}{c}\text { Exceso } \\
\text { Energía } \\
(\text { kWh) }\end{array}$ & $\begin{array}{c}\text { Ahorro } \\
\text { máximo } \\
\text { cons. } \\
\text { energético }\end{array}$ \\
\hline \multirow{3}{*}{ Sector 1} & 1 & 52 & 52 & \multirow{3}{*}{1,5} & \multirow{3}{*}{0,4} & \multirow{3}{*}{1,3} & \multirow{3}{*}{$72 \%$} & \multirow{3}{*}{0,5} & \multirow{3}{*}{$15 \%$} \\
\hline & 2 & 50 & 15 & & & & & & \\
\hline & 3 & 47 & 35 & & & & & & \\
\hline \multirow{2}{*}{ Sector 2} & 4 & 45 & 22 & \multirow{2}{*}{8,3} & \multirow{2}{*}{0,3} & \multirow{2}{*}{5,7} & \multirow{2}{*}{$67 \%$} & \multirow{2}{*}{2,8} & \multirow{2}{*}{$85 \%$} \\
\hline & 5 & 42 & 32 & & & & & & \\
\hline
\end{tabular}


Tabla 3.4 Escenario 1, alternativa presión 1.3 - Gestión de presión en el sector 1

\begin{tabular}{|c|c|c|c|c|c|c|c|c|c|}
\hline Sector & Nudos & $\underset{\text { (mca) }}{\mathrm{H}}$ & $\begin{array}{c}\mathbf{P} \\
\text { (mca) }\end{array}$ & $\begin{array}{c}\text { Energía } \\
\text { útil } \\
(\mathbf{k W h})\end{array}$ & $\begin{array}{c}\text { Energía } \\
\text { fugas } \\
\text { (kWh) }\end{array}$ & $\begin{array}{c}\text { Energía } \\
\text { mínima } \\
(\mathrm{kWh})\end{array}$ & $\begin{array}{l}\text { Eficiencia } \\
\text { energética }\end{array}$ & $\begin{array}{c}\text { Exceso } \\
\text { Energía } \\
\text { (kWh) }\end{array}$ & $\begin{array}{c}\text { Ahorro } \\
\text { máximo } \\
\text { cons. } \\
\text { energético }\end{array}$ \\
\hline \multirow{3}{*}{ Sector 1} & 1 & 70 & 70 & \multirow{3}{*}{5,2} & \multirow{3}{*}{0,6} & \multirow{3}{*}{2,7} & \multirow{3}{*}{$47 \%$} & \multirow{3}{*}{3,1} & \multirow{3}{*}{$79 \%$} \\
\hline & 2 & 68 & 33 & & & & & & \\
\hline & 3 & 65 & 53 & & & & & & \\
\hline \multirow{2}{*}{ Sector 2} & 4 & 38 & 15 & \multirow{2}{*}{5,0} & \multirow{2}{*}{0,1} & \multirow{2}{*}{4,3} & \multirow{2}{*}{$84 \%$} & \multirow{2}{*}{0,8} & \multirow{2}{*}{$21 \%$} \\
\hline & 5 & 35 & 25 & & & & & & \\
\hline
\end{tabular}

Tabla 3.5 Escenario 1, alternativa presión 1.4 - Gestión de presión en toda la red

\begin{tabular}{|c|c|c|c|c|c|c|c|c|c|}
\hline Sector & Nudos & $\underset{\text { (mca) }}{\mathrm{H}}$ & $\underset{\text { (mca) }}{P}$ & $\begin{array}{c}\text { Energía } \\
\text { útil } \\
(\mathbf{k W h})\end{array}$ & $\begin{array}{c}\text { Energía } \\
\text { fugas } \\
\text { (kWh) }\end{array}$ & $\begin{array}{c}\text { Energía } \\
\text { mínima } \\
(\mathrm{kWh})\end{array}$ & $\begin{array}{l}\text { Eficiencia } \\
\text { energética }\end{array}$ & $\begin{array}{c}\text { Exceso } \\
\text { Energía } \\
\text { (kWh) }\end{array}$ & $\begin{array}{c}\text { Ahorro } \\
\text { máximo } \\
\text { cons. } \\
\text { energético }\end{array}$ \\
\hline \multirow{3}{*}{ Sector 1} & 1 & 52 & 52 & \multirow{3}{*}{3,80} & \multirow{3}{*}{0,44} & \multirow{3}{*}{2,71} & \multirow{3}{*}{$64 \%$} & \multirow{3}{*}{1,5} & \multirow{3}{*}{$66 \%$} \\
\hline & 2 & 50 & 15 & & & & & & \\
\hline & 3 & 47 & 35 & & & & & & \\
\hline \multirow{2}{*}{ Sector 2} & 4 & 38 & 15 & \multirow{2}{*}{5} & \multirow{2}{*}{0} & \multirow{2}{*}{4} & \multirow{2}{*}{$84 \%$} & \multirow{2}{*}{0,8} & \multirow{2}{*}{$34 \%$} \\
\hline & 5 & 35 & 25 & & & & & & \\
\hline
\end{tabular}

A modo de resumen, se muestra una comparativa entre las cuatro alternativas dentro del primer escenario: la red sectorizada sin actuar sobre ella, gestión de presión únicamente sobre el sector 1, gestión de presión únicamente sobre el sector 2 y una gestión máxima de presión sobre toda la red.

Tabla 3.6 Comparativa de las diferentes alternativas de gestión de presiones - Escenario 1

\begin{tabular}{|c|c|c|c|c|c|c|}
\hline & $\begin{array}{c}\text { Presión } \\
\text { media }\end{array}$ & $\begin{array}{c}\text { Energía } \\
\text { útil (kWh) }\end{array}$ & $\begin{array}{c}\text { Energía } \\
\text { fugas } \\
\mathbf{( k W h )}\end{array}$ & $\begin{array}{c}\text { Energía } \\
\text { mínima } \\
\mathbf{( k W h )}\end{array}$ & $\begin{array}{c}\text { Eficiencia } \\
\text { energética }\end{array}$ & $\begin{array}{c}\text { Exceso de } \\
\text { Energía } \\
\mathbf{( k W h )}\end{array}$ \\
\hline Red sectorizada & 49,2 & 13,63 & 0,84 & 7,03 & $49 \%$ & 7,44 \\
\hline Gestión presión Sector 1 & 31,2 & 9,74 & 0,61 & 7,03 & $68 \%$ & 3,32 \\
\hline Gestión presión Sector 2 & 39,2 & 10,19 & 0,75 & 7,03 & $64 \%$ & 3,90 \\
\hline Gestión presión máxima & 28,4 & 8,78 & 0,59 & 7,03 & $75 \%$ & 2,33 \\
\hline
\end{tabular}

Una vez analizado el escenario anterior, se realizarán los mismos análisis, pero para la distribución sectorial del segundo escenario. En este escenario se englobarán en el sector 1 los nudos 1 y 2, y para el sector 2 los nudos 3,4 y 5 .

Según esta distribución (Tabla 3.7), el sector 2 presenta una peor eficiencia energética, lo que significa un mayor distanciamiento entre el nivel de energía disponible en el punto de consumo y el mínimo necesario, y por ende un mayor margen de mejora desde el punto de vista de la eficiencia. Ahora bien, entrando en términos absolutos de energía, es también el sector 2 el que presenta un exceso de energía mayor. 
Tabla 3.7 Escenario 2, alternativa presión 2.1 - Características energéticas de los sectores 1 y 2

\begin{tabular}{|c|c|c|c|c|c|c|c|c|c|}
\hline Sector & Nudos & $\underset{\text { (mca) }}{\mathrm{H}}$ & $\begin{array}{c}P \\
\text { (mca) }\end{array}$ & $\begin{array}{c}\text { Energía } \\
\text { útil } \\
\text { (kWh) }\end{array}$ & $\begin{array}{c}\text { Energía } \\
\text { fugas } \\
\text { (kWh) }\end{array}$ & $\begin{array}{c}\text { Energía } \\
\text { mínima } \\
(\mathbf{k W h})\end{array}$ & $\begin{array}{l}\text { Eficiencia } \\
\text { energética }\end{array}$ & $\begin{array}{c}\text { Exceso } \\
\text { de } \\
\text { Energía } \\
\text { (kWh) }\end{array}$ & $\begin{array}{c}\text { Ahorro max } \\
\text { cons. } \\
\text { Energético }\end{array}$ \\
\hline \multirow{2}{*}{ Sector 1} & 1 & 70 & 70 & \multirow{2}{*}{2,02} & \multirow{2}{*}{0,47} & \multirow{2}{*}{1,33} & \multirow{2}{*}{$53 \%$} & \multirow{2}{*}{1,16} & \multirow{2}{*}{$16 \%$} \\
\hline & 2 & 68 & 33 & & & & & & \\
\hline \multirow{3}{*}{ Sector 2} & 3 & 65 & 53 & \multirow{3}{*}{11,61} & \multirow{3}{*}{0,37} & \multirow{3}{*}{5,70} & \multirow{3}{*}{$48 \%$} & \multirow{3}{*}{6,27} & \multirow{3}{*}{$84 \%$} \\
\hline & 4 & 63 & 40 & & & & & & \\
\hline & 5 & 60 & 50 & & & & & & \\
\hline
\end{tabular}

Siguiendo con esta distribución de sectores, se procederá a implementar una gestión de presiones en ambos sectores. Se comenzará (Tabla 3.8) implantando una reducción de presión en el sector 1, con una única válvula reductora a la entrada del sector, de tal manera que se alcance la presión mínima admisible en el punto crítico de este sector. Así, el nudo crítico sería el nudo 2 y se podría reducir su presión hasta los $15 \mathrm{mca}$, viéndose afectados el resto de los nudos de la red. Acto seguido, y de igual manera, se analizará la alternativa de actuación para la gestión de presión 2.3, reduciendo la presión únicamente en el sector 2 (Tabla 3.9). En este caso, el nudo crítico es el 4 y su presión mínima quedará en 15 mca. En esta alternativa, solo se verán afectados los nudos del sector 2. Y finalmente, se analizará la última alternativa, con una reducción de presión máxima admisible en toda la red (Tabla 3.10), empleando sendas válvulas reductoras en cada entrada sectorial. Actuando sobre las dos entradas, es posible conseguir los mínimos niveles de presión en ambos sectores, en los nudos 2 y 4 .

Tabla 3.8 Escenario 2, alternativa presión 2.2 - Gestión de presión en el sector 1

\begin{tabular}{|c|c|c|c|c|c|c|c|c|c|}
\hline Sector & Nudos & $\underset{\text { (mca) }}{\mathrm{H}}$ & $\begin{array}{c}P \\
\text { (mca) }\end{array}$ & $\begin{array}{c}\text { Energía } \\
\text { útil } \\
(\mathbf{k W h})\end{array}$ & $\begin{array}{c}\text { Energía } \\
\text { fugas } \\
\text { (kWh) }\end{array}$ & $\begin{array}{c}\text { Energía } \\
\text { mínima } \\
(\mathrm{kWh})\end{array}$ & $\begin{array}{l}\text { Eficiencia } \\
\text { energética }\end{array}$ & $\begin{array}{l}\text { Exceso } \\
\text { de } \\
\text { Energía } \\
\text { (kWh) }\end{array}$ & $\begin{array}{c}\text { Ahorro max } \\
\text { cons. } \\
\text { Energético }\end{array}$ \\
\hline \multirow{2}{*}{ Sector 1} & 1 & 52 & 52 & \multirow{2}{*}{1,49} & \multirow{2}{*}{0,35} & \multirow{2}{*}{1,33} & \multirow{2}{*}{$72 \%$} & \multirow{2}{*}{0,51} & \multirow{2}{*}{$7 \%$} \\
\hline & 2 & 50 & 15 & & & & & & \\
\hline \multirow{3}{*}{ Sector 2} & 3 & 47 & 35 & \multirow{3}{*}{8,25} & \multirow{3}{*}{0,26} & \multirow{3}{*}{5,70} & \multirow{3}{*}{$67 \%$} & \multirow{3}{*}{2,81} & \multirow{3}{*}{$38 \%$} \\
\hline & 4 & 45 & 22 & & & & & & \\
\hline & 5 & 42 & 32 & & & & & & \\
\hline
\end{tabular}

Tabla 3.9 Escenario 2, alternativa presión 2.3 - Gestión de presión en el sector 2

\begin{tabular}{|c|c|c|c|c|c|c|c|c|c|}
\hline Sector & Nudos & $\underset{\text { (mca) }}{\mathrm{H}}$ & $\begin{array}{c}\mathrm{P} \\
\text { (mca) }\end{array}$ & $\begin{array}{c}\text { Energía } \\
\text { útil } \\
(\mathbf{k W h})\end{array}$ & $\begin{array}{c}\text { Energía } \\
\text { fugas } \\
\text { (kWh) }\end{array}$ & $\begin{array}{c}\text { Energía } \\
\text { mínima } \\
(\mathrm{kWh})\end{array}$ & $\begin{array}{c}\text { Eficiencia } \\
\text { energética }\end{array}$ & $\begin{array}{l}\text { Exceso } \\
\text { de } \\
\text { Energía } \\
\text { (kWh) }\end{array}$ & $\begin{array}{c}\text { Ahorro max } \\
\text { cons. } \\
\text { Energético }\end{array}$ \\
\hline \multirow{2}{*}{ Sector 1} & 1 & 70 & 70 & \multirow{2}{*}{2,02} & \multirow{2}{*}{0,47} & \multirow{2}{*}{1,33} & \multirow{2}{*}{$53 \%$} & \multirow{2}{*}{1,16} & \multirow{2}{*}{$16 \%$} \\
\hline & 2 & 68 & 33 & & & & & & \\
\hline \multirow{3}{*}{ Sector 2} & 3 & 40 & 28 & \multirow{3}{*}{6,95} & \multirow{3}{*}{0,22} & \multirow{3}{*}{5,70} & \multirow{3}{*}{$80 \%$} & \multirow{3}{*}{1,47} & \multirow{3}{*}{$20 \%$} \\
\hline & 4 & 38 & 15 & & & & & & \\
\hline & 5 & 35 & 25 & & & & & & \\
\hline
\end{tabular}


Tabla 3.10 Escenario 2, alternativa presión 2.4 - Gestión de presión máxima en toda la red

\begin{tabular}{|c|c|c|c|c|c|c|c|c|c|}
\hline Sector & Nudos & $\underset{\text { (mca) }}{\mathrm{H}}$ & $\begin{array}{c}P \\
\text { (mca) }\end{array}$ & $\begin{array}{c}\text { Energía } \\
\text { útil } \\
\text { (kWh) }\end{array}$ & $\begin{array}{c}\text { Energía } \\
\text { fugas } \\
\text { (kWh) }\end{array}$ & $\begin{array}{c}\text { Energía } \\
\text { mínima } \\
(\mathbf{k W h})\end{array}$ & $\begin{array}{l}\text { Eficiencia } \\
\text { energética }\end{array}$ & $\begin{array}{c}\text { Exceso } \\
\text { de } \\
\text { Energía } \\
\text { (kWh) }\end{array}$ & $\begin{array}{c}\text { Ahorro max } \\
\text { cons. } \\
\text { Energético }\end{array}$ \\
\hline \multirow{2}{*}{ Sector 1} & 1 & 52 & 52 & \multirow{2}{*}{1,49} & \multirow{2}{*}{0,35} & \multirow{2}{*}{1,33} & \multirow{2}{*}{$72 \%$} & \multirow{2}{*}{0,51} & \multirow{2}{*}{$7 \%$} \\
\hline & 2 & 50 & 15 & & & & & & \\
\hline \multirow{3}{*}{ Sector 2} & 3 & 40 & 28 & \multirow{3}{*}{6,95} & \multirow{3}{*}{0,22} & \multirow{3}{*}{5,70} & \multirow{3}{*}{$80 \%$} & \multirow{3}{*}{1,47} & \multirow{3}{*}{$20 \%$} \\
\hline & 4 & 38 & 15 & & & & & & \\
\hline & 5 & 35 & 25 & & & & & & \\
\hline
\end{tabular}

A modo de resumen, se muestra una comparativa entre las cuatro alternativas dentro del primer escenario: la red sectorizada sin actuar sobre ella, gestión de la presión únicamente sobre el sector 1 , gestión de presión únicamente sobre el sector 2 y una gestión máxima de presión sobre toda la red.

Tabla 3.11 Comparativa de las diferentes alternativas de gestión de presiones - Escenario 2

\begin{tabular}{|c|c|c|c|c|c|c|}
\hline & $\begin{array}{c}\text { Presión } \\
\text { media }\end{array}$ & $\begin{array}{c}\text { Energía } \\
\text { útil (kWh) }\end{array}$ & $\begin{array}{c}\text { Energía } \\
\text { fugas } \\
\mathbf{( k W h )}\end{array}$ & $\begin{array}{c}\text { Energía } \\
\text { mínima } \\
\mathbf{( k W h )}\end{array}$ & $\begin{array}{c}\text { Eficiencia } \\
\text { energética }\end{array}$ & $\begin{array}{c}\text { Exceso de } \\
\text { Energía } \\
\mathbf{( k W h )}\end{array}$ \\
\hline Red sectorizada & 49,2 & 13,63 & 0,84 & 7,03 & $49 \%$ & 7,44 \\
\hline Gestión presión Sector 1 & 31,2 & 9,74 & 0,61 & 7,03 & $68 \%$ & 3,32 \\
\hline Gestión presión Sector 2 & 34,2 & 8,97 & 0,70 & 7,03 & $73 \%$ & 2,63 \\
\hline Gestión presión máxima & 27 & 8,44 & 0,57 & 7,03 & $78 \%$ & 1,98 \\
\hline
\end{tabular}

Como era de esperar, los resultados obtenidos en ambos escenarios de sectorización son bien distintos. En el escenario 1, se desprende que la menor eficiencia energética se encuentra en el sector 1 y que la reducción máxima de energía se producirá en el sector 2 (42\% en el sector 1 frente al 58\% en segundo sector). En cambio, en el escenario 2, ha sido el sector 2 el que cuenta con un menor rendimiento energético y un mayor ahorro de energía posible (84\% frente al $16 \%$ que presenta el sector 1$)$. Comparando ambos escenarios, el menor rendimiento energético se da en el sector 1 del escenario 1 y el mayor ahorro posible se obtendrá con el sector 2 del segundo escenario. Una vez sectorizada la red, los mejores resultados tras la gestión de la presión se han obtenido con la sectorización planteada en el escenario 2.

De todo ello, se desprende que la definición de la sectorización que ha permitido conseguir mejores resultados para la gestión activa de fugas o menores consumos energéticos, a priori, es aquella que permita una mayor reducción de la energía útil y energía de fugas. Y, como se ha visto en el ejemplo, no tiene por qué coincidir con una menor eficiencia energética.

Esto es debido a que este indicador, según lo definen Boulos \& Bross (2010) o Cabrera et al. (2010), se obtiene con la altura piezométrica (dependiendo de la cota, presión y velocidad). Despreciando la velocidad, la cota puede aportar un mayor valor que la presión y por ello un mayor peso en el cálculo de la energía en el punto de consumo. Por lo tanto, para la evaluación de los sectores será más apropiado calcular la misma eficiencia energética, pero considerando únicamente el término de la energía aportado por la presión en el punto de consumo. Se le ha denominado a este indicador eficiencia energética dinámica y se calcularía según la siguiente expresión: 


$$
\eta_{e d}=\frac{E_{u o}}{E_{t}}=\frac{\gamma \cdot \sum_{t_{k}=t_{1}}^{t_{k}=t_{p}}\left(\sum_{k=1}^{n} q_{k}\left(t_{k}\right) \cdot{\frac{P_{\min }}{\gamma}}_{k}\right) \cdot \Delta t}{\gamma \cdot \sum_{t_{k}=t_{1}}^{t_{k}=t_{p}}\left(\sum_{k=1}^{n} q_{k}\left(t_{k}\right) \cdot{\frac{P_{k}}{\gamma_{k}}}_{k}\right) \cdot \Delta t}
$$

donde $E_{u o}$ es la energía mínima requerida que debe suministrarse a los puntos de consumo para cumplir con la presión mínima establecida y $E_{t}$ es toda la energía suministrada a los puntos de consumo.

Se podría caer en la equivocación de suponer que evaluar o guiar el diseño de una sectorización según la eficiencia energética dinámica es equivalente a realizarlo siguiendo los niveles de presión. Y este pequeño matiz puede suponer una diferencia importante según el tipo de sistema. En aquellos donde la energía suministrada es totalmente energía natural (por gravedad), aportada por un depósito de cabecera, el ahorro de energía puede ser secundario frente a la disminución de presión. En cambio, en sistemas alimentados por grupos de presión, esta apreciación puede ser muy importante de cara a reducciones de consumos energéticos. Véase para ello la red de la Figura 3.3a, la cual representa a dos nudos independientes, $i$ y $j$, los cuales se suministran por medio de un grupo de impulsión. El nudo $i$ cuenta con una presión de 27 mca y el nudo j de 50 mca, estando marcado el nivel de presión mínima en 25 mca. Respecto al consumo, se plantea un primer escenario (Tabla 3.12) donde se dotará al nudo $i$ con un consumo de $1.000 \mathrm{l} / \mathrm{s}$ y al nudo $j$ con un consumo de $1 \mathrm{l} / \mathrm{s}$, y un segundo escenario (Tabla 3.13) donde se intercambian los consumos. Y sobre la red, se pretende implantar una gestión de presiones.
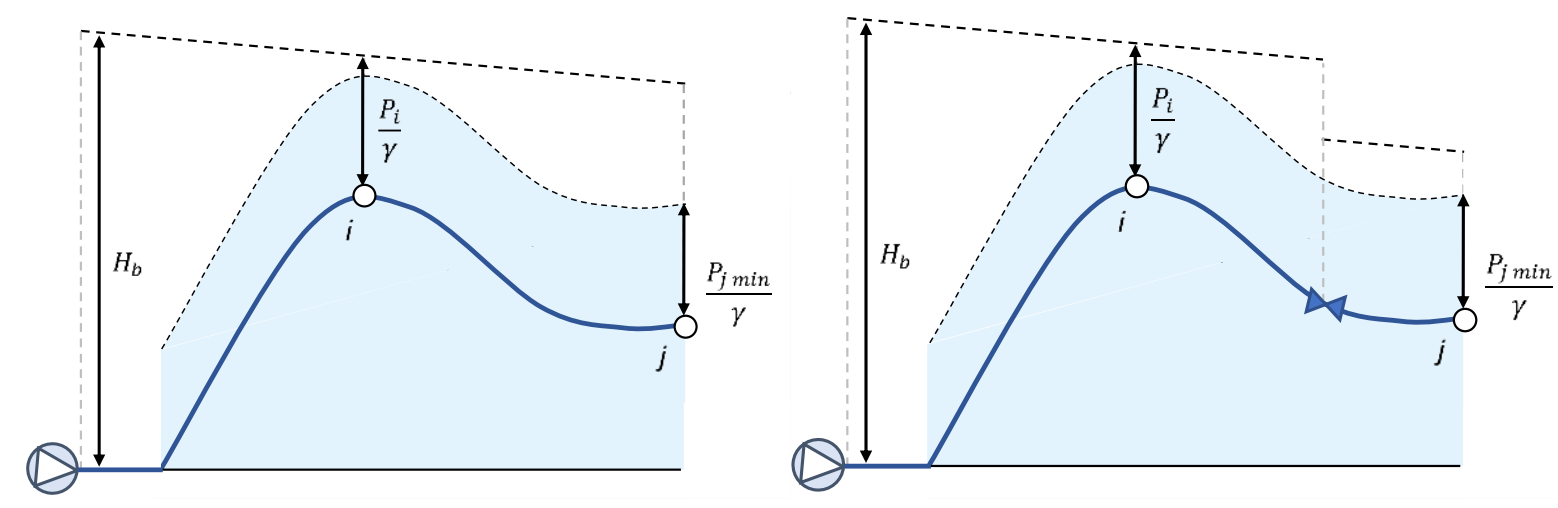

Figura 3.3. Red de distribución con grupo de presión. Sin gestión de presión (a) y con gestión de presión (b)

Una gestión clásica de presiones orientaría los trabajos hacía una reducción de la presión en el nudo $j$ (Figura 3.3b), dejando en un segundo plano la presión del sector $i$, por estar muy próxima a la presión mínima. Para el primer escenario (Tabla 3.12), si se analiza la componente energética del problema, se puede observar cómo cada mca disminuido en la presión del nudo j, significará una reducción del 0,004\% del total de la energía consumida. En cambio, una reducción de tan solo 1 mca en el nudo $i$, conseguirá una reducción del 3,7\%. En el segundo caso (Tabla 3.13), los números son bien distintos. Una disminución de 1 mca en la presión del nudo j conllevará una reducción del 3,7\% en el consumo energético total. Y de un $0,007 \%$ la reducción de 1 mca en la presión del nudo $i$. Por todo ello, se puede afirmar que la eficiencia energética dinámica es un indicador más acertado para la evaluación de la eficiencia energética del sistema. 
Tabla 3.12 Escenario 1 de consumos de la red de la Figura 3.3

\begin{tabular}{|c|c|c|c|c|c|c|c|c|}
\hline Sectores & $\begin{array}{c}\mathbf{P} \\
\text { (mca) }\end{array}$ & $\begin{array}{c}\mathbf{P} \text { min } \\
\text { (mca) }\end{array}$ & $\begin{array}{c}\text { Consumo } \\
\text { (Ips) }\end{array}$ & $\begin{array}{c}\text { Energía } \\
\text { útil } \\
\mathbf{( k W h )}\end{array}$ & $\begin{array}{c}\text { Energía } \\
\text { mínima } \\
\mathbf{( k W h )}\end{array}$ & $\begin{array}{c}\text { Eficiencia } \\
\text { energética de } \\
\text { presión }\end{array}$ & $\begin{array}{c}\text { Exceso de } \\
\text { Energía } \\
\text { (kWh) }\end{array}$ & $\begin{array}{c}\text { Ahorro max } \\
\text { cons. } \\
\text { Energético }\end{array}$ \\
\hline$j$ & 50 & 25 & 1 & 0,49 & 0,25 & & 0,25 & $1,2 \%$ \\
\hline$i$ & 27 & 25 & 1000 & 264,87 & 245,25 & $93 \%$ & 19,62 & $98,8 \%$ \\
\hline
\end{tabular}

Tabla 3.13 Escenario 2 de consumos de la red de la Figura 3.3

\begin{tabular}{|c|c|c|c|c|c|c|c|c|}
\hline Sectores & $\begin{array}{c}\mathbf{P} \\
\text { (mca) }\end{array}$ & $\begin{array}{c}\mathbf{P} \text { min } \\
\text { (mca) }\end{array}$ & $\begin{array}{c}\text { Consumo } \\
\text { (Ips) }\end{array}$ & $\begin{array}{c}\text { Energía } \\
\text { útil } \\
\mathbf{( k W h )}\end{array}$ & $\begin{array}{c}\text { Energía } \\
\text { mínima } \\
\mathbf{( k W h )}\end{array}$ & $\begin{array}{c}\text { Eficiencia } \\
\text { energética de } \\
\text { presión }\end{array}$ & $\begin{array}{c}\text { Exceso de } \\
\text { Energía } \\
\text { (kWh) }\end{array}$ & $\begin{array}{c}\text { Ahorro max } \\
\text { cons. } \\
\text { Energético }\end{array}$ \\
\hline$j$ & 50 & 25 & 1000 & 490,50 & 245,25 & & 245,25 & $99,99 \%$ \\
\hline$i$ & 27 & 25 & 1 & 0,26 & 0,25 & $50 \%$ & 0,02 & $0,01 \%$ \\
\hline
\end{tabular}

\subsubsection{Planteamiento de la metodología}

Por todo lo visto a lo largo de este capítulo, abordar el diseño de las sectorizaciones según criterios energéticos, más concretamente, según niveles de energía, se puede considerar un factor clave de cara a futuros ahorros energéticos. Con esta base, a continuación, se presenta una metodología semi autónoma que ayudará al diseñador en la fase de diseño de las sectorizaciones de las redes de distribución de agua.

La metodología propuesta consiste en un procedimiento de localización y agrupación de aquellas zonas con menor eficiencia energética dinámica. Es decir, se plantea un procedimiento de agrupación de los elementos de la red, de tal forma que la diferencia entre el nivel mínimo de energía disponible para los consumidores en forma de presión y la real entregada sea la menor posible. De manera analítica, se pretende agrupar los nudos en diferentes grupos con el objetivo de alcanzar el valor mínimo de la siguiente función objetivo:

$$
\text { F.O. } \min \sum_{j=1}^{N_{c}} \sum_{i=1}^{n} a b s\left(\eta_{e d_{i}}-c_{j}\right) a_{i j}
$$

donde $N_{c}$ representa el número de clusters o sectores, $n$ el número nudos, $\mathrm{a}_{\mathrm{ij}}$ tomará el valor de 1 si el elemento pertenece al sector y cero en el resto de los casos, $\eta_{e d_{i}}$ corresponde con la eficiencia energética dinámica del nudo $i$ (ecuación 3.9) y $c_{\mathrm{j}}$ será la eficiencia energética dinámica de los nudos que forman el sector $j$.

La resolución de la ecuación 3.10 puede llevarse a cabo con cualquier método de resolución heurístico, como por ejemplo algoritmos genéticos. En el presente trabajo se ha optado por la vía que brinda la teoría de grafos, siguiendo los conceptos recogidos en el segundo capítulo.

Para la resolución adoptada, en primer lugar, y para poder abordar los aspectos topológicos y de conectividad, la red de distribución se considera un grafo no dirigido (Kesavan and Chandrashekar 1972; Gupta and Prasad 2000), es decir, aquel en que las líneas no tienen una dirección definida (o lo que es lo mismo, no se sabe a priori la dirección del caudal). Asimismo, los nudos de consumo de la red de distribución son los vértices. En segundo lugar, y debido a la complejidad y volumen de cálculos a realizar, es preciso emplear un sistema 
multi-agente. Entiéndase un agente como un sistema capaz de actuar independientemente según los cambios en el medio e interactuar con otros (Maturana et. al., 2006). Por último, se recurrirá al algoritmo de Prim (Prim, 1957) para encontrar el árbol recubridor mínimo del grafo conexo (la red), no dirigido y cuyas aristas (los nudos de consumo) están etiquetadas.

Así, la metodología planteada se encargará de encontrar aquella zona en la red donde la variable elegida, en este caso el rendimiento energético dinámico, sea menor. Para ello, el proceso de cálculo barrerá la red de manera ordenada, empezando por el nudo establecido y guiado por los niveles mínimos de eficiencia energética dinámica de los elementos a barrer. Una vez seleccionada una zona, esta quedará eliminada, y se volverá a ejecutar el proceso para el resto de red. Finalmente, se obtendrán diferentes zonas agrupadas según diferentes niveles de eficiencia energética dinámica.

Para mayor comprensión de la metodología, a continuación, se detallan los pasos a seguir y el diagrama de flujo de dicho procedimiento. Este ejemplo partirá de una sencilla red mallada, compuesta por 8 nudos de consumo (identificados con letras) y 10 líneas (identificadas con números). Evidentemente, cada uno de los nudos tendrá un valor de presión y cada línea un valor de caudal, pudiendo así calcular la eficiencia energética dinámica para cada grupo de elementos, aunque para el objetivo que perseguimos el valor en sí no es relevante.

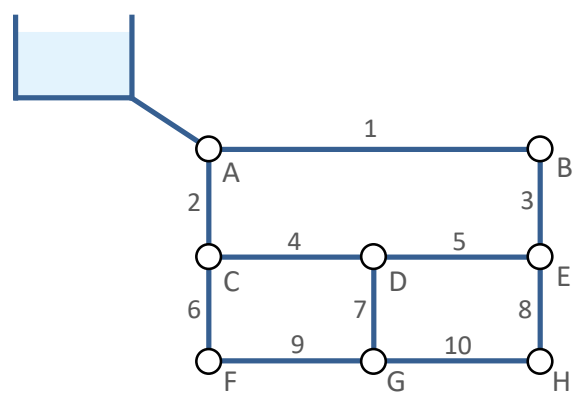

Figura 3.4. Red de distribución suministrada por un depósito de cabecera

Arbitrariamente, se ha cogido el nudo F como punto de partida. Automáticamente, este nudo queda englobado en el clúster de cálculo. Para el grupo de cálculo definido en el paso 1, el rendimiento energético dinámico tomará un valor de $\eta_{e d_{1}}$. A su vez y de cara al siguiente paso, es necesario marcar los nudos frontera con los nudos englobados en el clúster. Así, los nudos directamente conectados con F son C-6 y G-9.

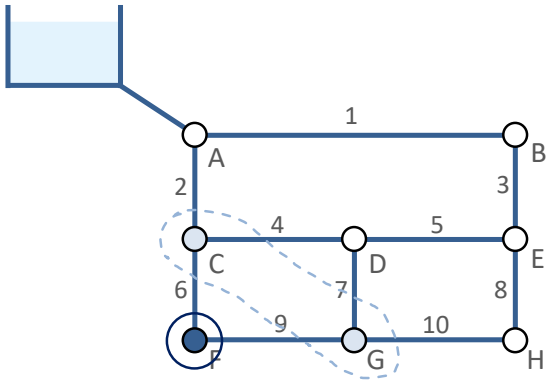

Figura 3.5. Paso 1

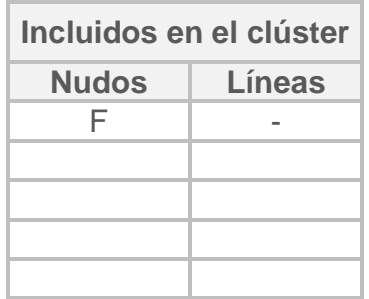

\begin{tabular}{|c|c|}
\hline \multicolumn{2}{|c|}{ Frontera } \\
\hline Nudos & Líneas \\
\hline C & 6 \\
\hline G & 9 \\
\hline & \\
\hline & \\
\hline & \\
\end{tabular}

eficiencia energética dinámica $\eta_{e d_{1}}$

En el paso 2, se incluirá en el clúster al nudo que menor eficiencia energética dinámica tenga. Supongamos que corresponde con el nudo G. Ahora, el clúster está formado por los nudos $\mathrm{F}$ 
y $G$ y la línea 9 , y $\eta_{e d_{2}}$ es el valor de la eficiencia energética dinámica. En cuanto a los nudos frontera, estos serán los C-6, H-10 y D-7.

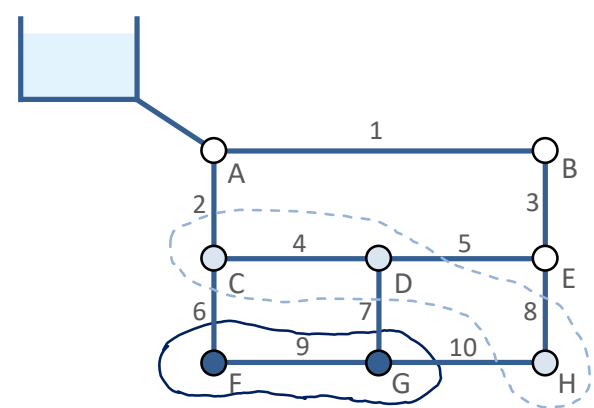

Figura 3.6. Paso 2

\begin{tabular}{|c|c|}
\hline \multicolumn{2}{|c|}{ Incluidos en el clúster } \\
\hline Nudos & Líneas \\
\hline F & - \\
\hline G & 9 \\
\hline & \\
\hline & \\
\hline & \\
\end{tabular}

\begin{tabular}{|c|c|}
\hline \multicolumn{2}{|c|}{ Frontera } \\
\hline Nudos & Líneas \\
\hline C & 6 \\
\hline H & 10 \\
\hline D & 7 \\
\hline & \\
\hline & \\
\hline
\end{tabular}

eficiencia energética dinámica

\section{$\eta_{e d}$}

En el paso 3, el nudo D será incluido en el clúster, siendo $\eta_{e d_{3}}$ el valor de la eficiencia energética dinámica del clúster actualizado. Ahora el grupo de cálculo está formado por los nudos F, G y D. En cuanto a los nudos frontera, estos serán el C-6-4, H-10 y E-5.

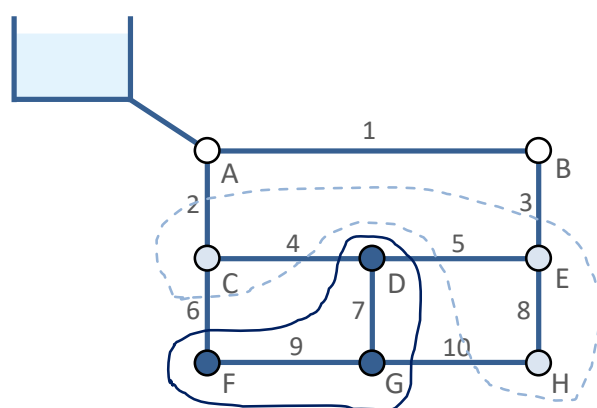

Figura 3.7. Paso 3

\begin{tabular}{|c|c|}
\hline \multicolumn{2}{|c|}{ Incluidos en el clúster } \\
\hline Nudos & Líneas \\
\hline F & - \\
\hline G & 9 \\
\hline D & 7 \\
\hline & \\
\hline & \\
\hline
\end{tabular}

\begin{tabular}{|c|c|}
\hline \multicolumn{2}{|c|}{ Frontera } \\
\hline Nudos & Líneas \\
\hline C & 6 \\
\hline & 4 \\
\hline H & 10 \\
\hline E & 5 \\
\hline & \\
\hline
\end{tabular}

eficiencia energética dinámica

\begin{tabular}{|c|c|}
\hline \multicolumn{2}{|c|}{ Incluidos en el clúster } \\
\hline Nudos & Líneas \\
\hline F & - \\
\hline G & 9 \\
\hline D & 7 \\
\hline C & 6 \\
\hline C & 4 \\
\hline
\end{tabular}

\section{eficiencia energética} dinámica

\begin{tabular}{|c|c|}
\hline \multicolumn{2}{|c|}{ Frontera } \\
\hline Nudos & Líneas \\
\hline A & 2 \\
\hline H & 10 \\
\hline D & 7 \\
\hline & \\
\hline & \\
\hline
\end{tabular}

$\eta_{e d_{4}}$

Figura 3.8. Paso 4

El proceso de barrido continuará hasta que todos los nudos queden incluidos en el clúster. En este caso, el algoritmo de búsqueda ha empezado por el nudo $\mathrm{F}$ y terminará tras 8 pasos (uno por cada nudo). Para cada paso, y tras incluir en el clúster un nuevo nudo, el valor de la eficiencia energética dinámica del clúster se irá actualizando (Figura 3.9). De esta forma, la eficiencia tomará un valor mínimo en alguno de los pasos, siendo los elementos englobados en el clúster, en ese momento, la zona con menor eficiencia energética dinámica y la que 
presentará, a priori, un mayor margen de mejora. En este caso, la menor eficiencia energética dinámica se alcanza en el paso 4. Así, los nudos F, G, D y C y las líneas 9, 6, 4 y 7 engloban la zona con menor eficiencia energética dinámica de toda la red. El resto de los nudos $(A, B$, E y H) quedarán englobados en otra zona con una eficiencia energética mayor. Sobre esta zona se podrá lanzar de nuevo el algoritmo de búsqueda, si se quiere seguir dividiendo.

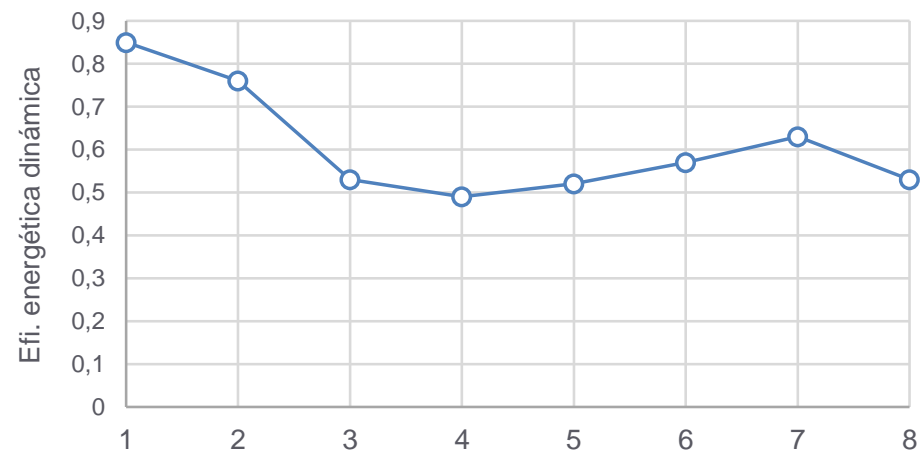

Figura 3.9. Evolución de la eficiencia energética dinámica para cada paso

Una vez ejecutado el algoritmo y obtenidas las zonas resultantes, estas no se pueden entender como sectores finales debido a que no se encuentran aislados entre sí. Pero sí constituyen una buena aproximación a la delimitación de los futuros sectores. Para la definición final, será necesario considerar cuáles son las tuberías de interconexión que deben ser cerradas para aislar el sector (por medio de procesos heurísticos, por ejemplo).

Para terminar con el desarrollo de la metodología y siguiendo con el ejemplo, cabría destacar dos aspectos importantes. En primer lugar, el algoritmo de barrido empezaba en el nudo $\mathrm{F} \mathrm{e}$ avanza nudo a nudo hasta tener toda la red barrida. Una vez terminado, se busca qué grupos de nudos presentan el menor valor de eficiencia energética dinámica. Así, se obtiene la zona con menor eficiencia, empezando el barrido por el nudo F. Evidentemente, si el algoritmo empieza por otro nudo, la zona con menor eficiencia no tiene por qué coincidir. Por ello, si se quiere localizar el mínimo global y no local, será necesario que el algoritmo busque en todas las combinaciones posibles. En segundo lugar, y siguiendo en la misma línea de esta última idea, puede darse el caso de que sea necesario poner restricciones al diseño, y por lo tanto el algoritmo dará como resultado un mínimo local. Por todo ello, la metodología propuesta precisa de unas condiciones mínimas para su correcto funcionamiento, que determinarán el inicio del algoritmo y las condiciones que deberán cumplir las zonas obtenidas como resultado. Según la definición de los vértices y las condiciones de contorno, se plantean dos modalidades de funcionamiento que se desarrollan en los siguientes apartados: diseño con y sin restricciones.

\subsubsection{Diseño con restricciones}

Siguiendo con las premisas anteriores, se plantea en este apartado el primer procedimiento de optimización denominado Diseño con restricciones. Se caracterizará, principalmente, por el hecho de que el desarrollo del algoritmo de cálculo quedará condicionado por diferentes condiciones de contorno fijadas al comienzo de este. Por ejemplo, se pueden fijar el número de sectores, la ubicación de estos o una longitud mínima o máxima de tuberías englobadas en cada sector. 
Una vez establecidos los puntos de partida, el algoritmo se ejecutará un número de veces igual al número de sectores, buscará la zona de menor rendimiento energético dinámico y la excluirá del grupo de cálculo. Una vez realizado esto, y con un sector predefinido menos, se volverá a ejecutar hasta que se completen todos los sectores marcados.

En la Figura 3.10 se muestra el diagrama de flujo de los pasos a seguir para la aplicación de este algoritmo.

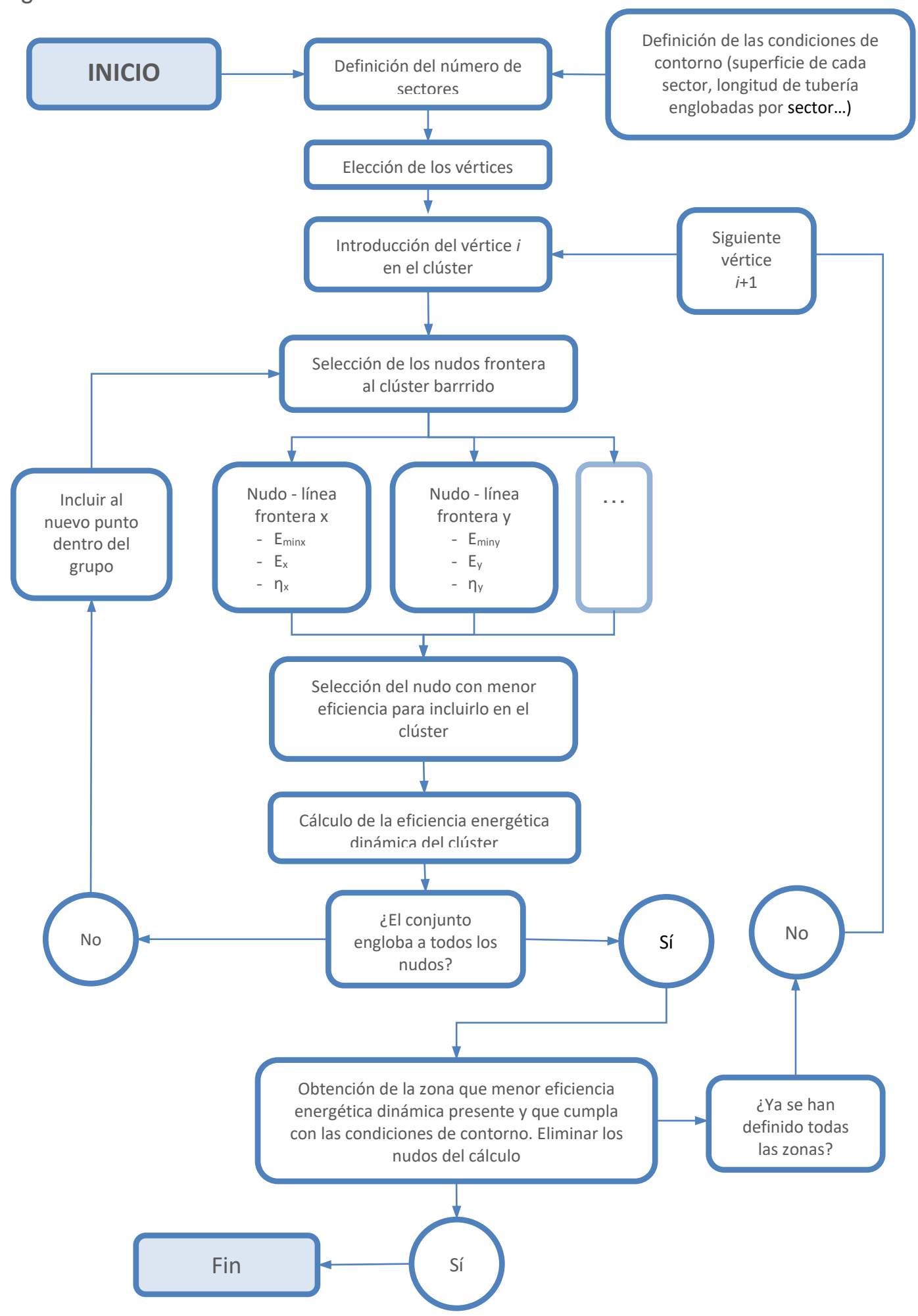

Figura 3.10. Diagrama de flujo del procedimiento de diseño con restricciones 


\subsubsection{Ejemplo numérico}

Para una mejor compresión de la metodología desarrollada, ésta se aplicará sobre la red sintética mostrada en la Figura 3.11. La red tiene una longitud total de $50 \mathrm{~km}$ con 58 tuberías que suministran a 38 nodos de consumo cuya elevación, por simplicidad, es cero. Unos 6.500 $\mathrm{m}^{3}$ de agua se entregan diariamente (40.000 habitantes). Ambos embalses están elevados 50 $\mathrm{m}$. La rugosidad de todas las tuberías es de $0,1 \mathrm{~mm}$, y las longitudes y diámetros de las tuberías se muestran en la Tabla 3.14

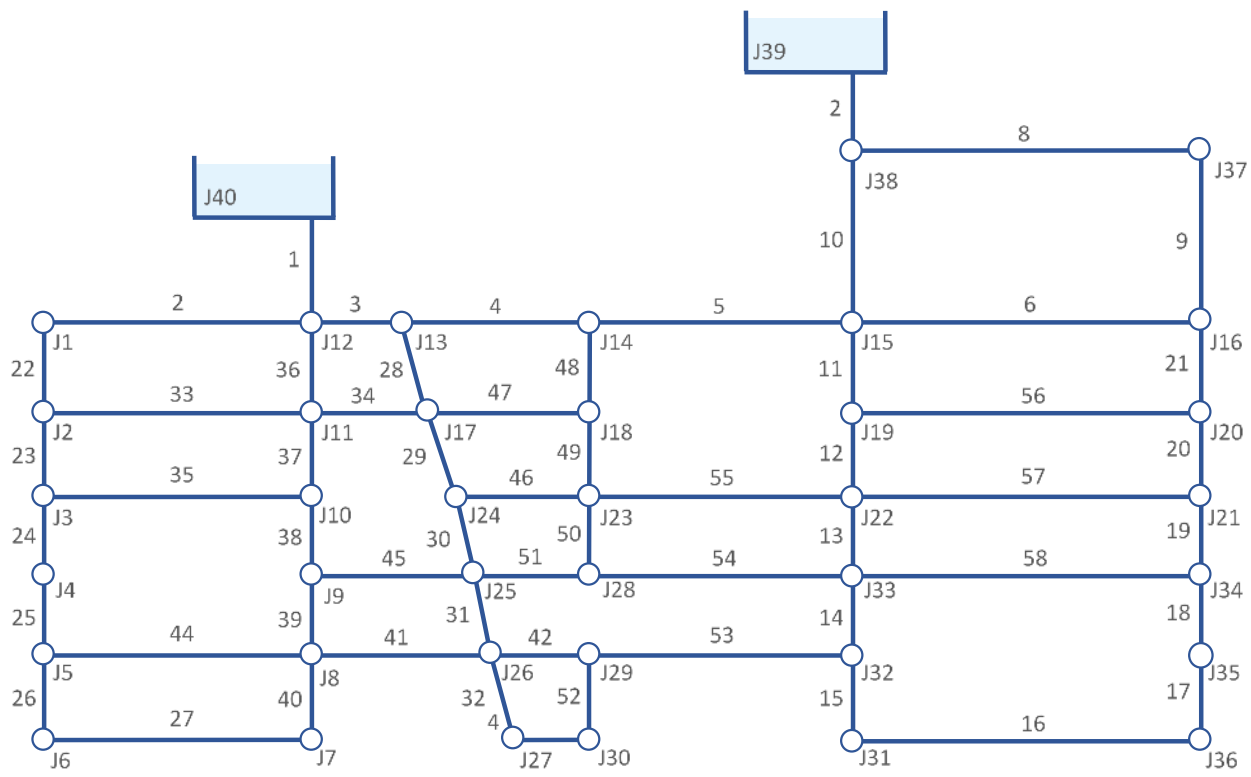

Figura 3.11. Diseño de la red de distribución.

La demanda base es de $60 \mathrm{l} / \mathrm{min}\left(0,001 \mathrm{~m}^{3} / \mathrm{s}\right)$ para cada uno de los nudos entre el 1 y $16, \mathrm{y}$ también para los nudos 21, 27 y 30, mientras que, para los 19 nudos restantes, la demanda base es de $180 \mathrm{l} / \mathrm{min}(0,003 \mathrm{~m} / \mathrm{s})$. Para no dotar de complejidad al caso de estudio, debido a que el objetivo es el de observar el resultado de la metodología, se ha supuesto un rendimiento hidráulico de la red del 100\%, con lo que no es necesario estimar caudales de fugas nodales. Tampoco se ha dotado de modulación horaria para el consumo de los nudos, por la misma razón.

En cuanto a las tuberías, se tiene una red arterial primaria formada por tuberías de 150 y 200 $\mathrm{mm}$ y una red arterial secundaria de $100 \mathrm{~mm}$. La red de distribución está formada por tuberías de 90 y $50 \mathrm{~mm}$. A continuación, se detallan los diámetros de cada una de ellas. 


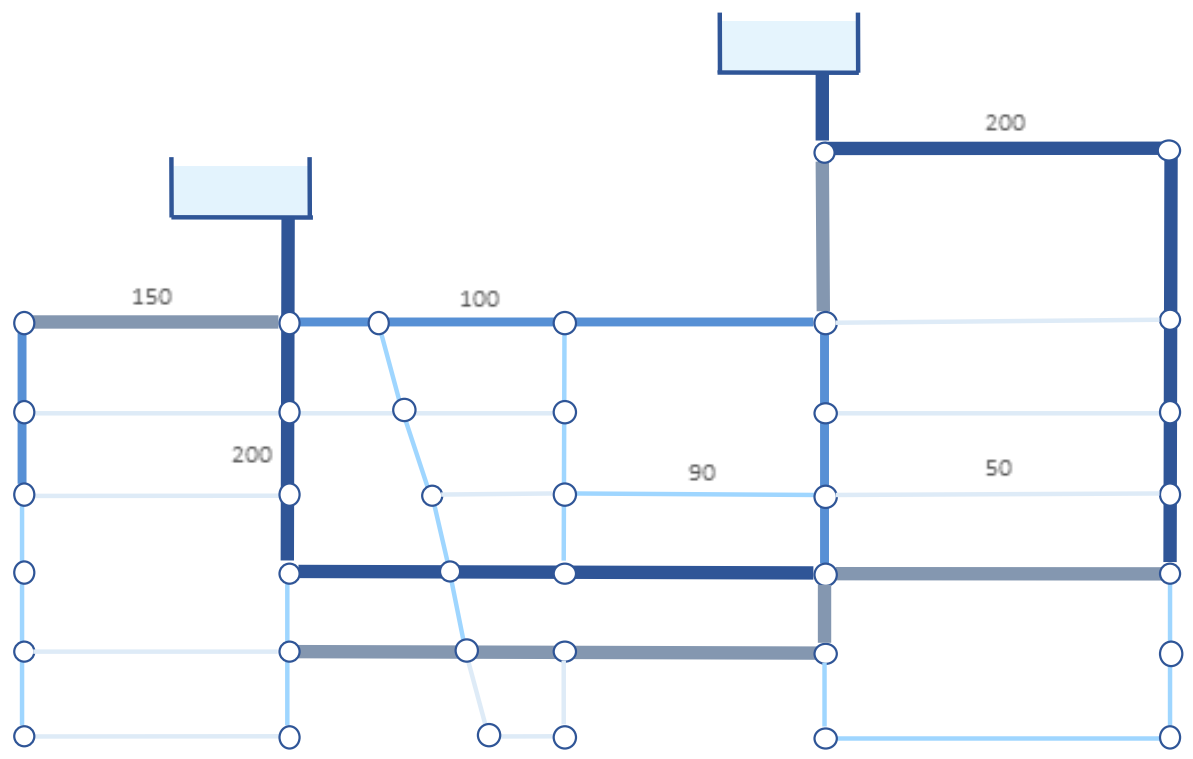

Figura 3.12. Distribución de calibres en las tuberías

En su estado operativo normal, la presión promedio es de 37,7 mca, con valores extremos de 31,7 y 47,0 mca (Tabla 4.1). Los valores promedio de velocidad del agua y pérdida de carga unitaria en las tuberías son $0,41 \mathrm{~m} / \mathrm{s}$ y $2,50 \mathrm{mca} / \mathrm{km}$, respectivamente (Tabla 3.14). La procedencia del caudal es, aproximadamente, del 50\% de cada depósito.

Tabla 3.14 Características físicas y valores de operación normal para variables de tuberías

\begin{tabular}{|c|c|c|c|c|c|}
\hline ID & $\begin{array}{c}\text { Diám. } \\
(\mathrm{mm})\end{array}$ & $\begin{array}{c}\text { Longitud } \\
(\mathrm{m})\end{array}$ & $\begin{array}{c}\text { Caudal } \\
(\mathrm{I} / \mathrm{min})\end{array}$ & $\begin{array}{c}\mathrm{V} . \\
(\mathrm{m} / \mathrm{s})\end{array}$ & $\begin{array}{c}\text { Pérdidas } \\
\text { unitarias } \\
(\mathrm{m} / \mathrm{km})\end{array}$ \\
\hline 1 & 200 & 977,3 & 2298,0 & 1,2 & 7,1 \\
\hline 2 & 150 & 1629,5 & 330,6 & 0,3 & 0,8 \\
\hline 3 & 100 & 559,4 & 345,0 & 0,7 & 6,4 \\
\hline 4 & 100 & 1021,6 & 67,8 & 0,1 & 0,3 \\
\hline 5 & 100 & 1624,3 & 219,0 & 0,5 & 2,7 \\
\hline 6 & 50 & 1862,8 & 25,8 & 0,2 & 1,7 \\
\hline 7 & 200 & 428,1 & 2262,0 & 1,2 & 6,9 \\
\hline 8 & 200 & 1838,5 & 1341,6 & 0,7 & 2,6 \\
\hline 9 & 200 & 986,9 & 1161,6 & 0,6 & 2 \\
\hline 10 & 150 & 987,9 & 740,4 & 0,7 & 3,5 \\
\hline 11 & 100 & 481,3 & 435,6 & 0,9 & 10 \\
\hline 12 & 100 & 510,8 & 266,4 & 0,6 & 4 \\
\hline 13 & 100 & 432,7 & 70,2 & 0,2 & 0,4 \\
\hline 14 & 150 & 461,7 & 531,6 & 0,5 & 1,9 \\
\hline 15 & 90 & 467,7 & 222,0 & 0,6 & 4,8 \\
\hline 16 & 90 & 1830,3 & 42,0 & 0,1 & 0,2 \\
\hline 17 & 90 & 447,1 & 138,0 & 0,4 & 2 \\
\hline 18 & 90 & 464,2 & 318,0 & 0,8 & 9,3 \\
\hline 19 & 200 & 471,8 & 856,2 & 0,5 & 1,1 \\
\hline 20 & 200 & 482,7 & 936,6 & 0,5 & 1,3 \\
\hline
\end{tabular}

\begin{tabular}{|c|c|c|c|c|c|}
\hline ID & $\begin{array}{l}\text { Diám. } \\
(\mathrm{mm})\end{array}$ & $\begin{array}{l}\text { Longitud } \\
\text { (m) }\end{array}$ & $\begin{array}{l}\text { Caudal } \\
\text { (I/min) }\end{array}$ & $\begin{array}{c}\text { V. } \\
(\mathrm{m} / \mathrm{s})\end{array}$ & $\begin{array}{c}\text { Pérdidas } \\
\text { unitarias } \\
(\mathrm{m} / \mathrm{km})\end{array}$ \\
\hline 30 & 90 & 498,5 & 106,2 & 0,3 & 1,2 \\
\hline 31 & 90 & 426,1 & 194,4 & 0,5 & 3,7 \\
\hline 32 & 50 & 471,4 & 60,6 & 0,5 & 8 \\
\hline 33 & 50 & 1634,9 & 18,6 & 0,2 & 1 \\
\hline 34 & 50 & 775,2 & 51,0 & 0,4 & 5,8 \\
\hline 35 & 50 & 1648,6 & 19,2 & 0,2 & 1 \\
\hline 36 & 200 & 496 & 1561,8 & 0,8 & 3,4 \\
\hline 37 & 200 & 476,3 & 1432,2 & 0,8 & 2,9 \\
\hline 38 & 200 & 482,7 & 1352,4 & 0,7 & 2,6 \\
\hline 39 & 90 & 437,4 & 267,0 & 0,7 & 6,7 \\
\hline 40 & 90 & 512,6 & 55,8 & 0,2 & 0,4 \\
\hline 41 & 150 & 1064,9 & 156,0 & 0,2 & 0,2 \\
\hline 42 & 150 & 497,6 & 109,2 & 0,1 & 0,1 \\
\hline 43 & 50 & 485,2 & 0,6 & 0 & 0 \\
\hline 44 & 50 & 1652,9 & 4,2 & 0 & 0,1 \\
\hline 45 & 200 & 1012,2 & 1025,4 & 0,5 & 1,6 \\
\hline 46 & 50 & 699,9 & 6,0 & 0 & 0,1 \\
\hline 47 & 50 & 797,1 & 9,0 & 0,1 & 0,3 \\
\hline 48 & 90 & 501,4 & 226,8 & 0,6 & 4,9 \\
\hline 49 & 90 & 456,9 & 55,8 & 0,2 & 0,4 \\
\hline
\end{tabular}




\begin{tabular}{|c|c|c|c|c|c|}
\hline ID & $\begin{array}{c}\text { Diám. } \\
(\mathrm{mm})\end{array}$ & $\begin{array}{c}\text { Longitud } \\
(\mathrm{m})\end{array}$ & $\begin{array}{c}\text { Caudal } \\
(\mathrm{l} / \mathrm{min})\end{array}$ & $\begin{array}{c}\mathrm{V} . \\
(\mathrm{m} / \mathrm{s})\end{array}$ & $\begin{array}{c}\text { Pérdidas } \\
\text { unitarias } \\
(\mathrm{m} / \mathrm{km})\end{array}$ \\
\hline 21 & 200 & 520,3 & 1127,4 & 0,6 & 1,9 \\
\hline 22 & 100 & 486,6 & 270,6 & 0,6 & 4,1 \\
\hline 23 & 100 & 496 & 229,2 & 0,5 & 3 \\
\hline 24 & 90 & 461,7 & 189,0 & 0,5 & 3,5 \\
\hline 25 & 90 & 473,4 & 129,0 & 0,3 & 1,7 \\
\hline 26 & 90 & 471,8 & 64,2 & 0,2 & 0,5 \\
\hline 27 & 50 & 1625,4 & 4,2 & 0 & 0,1 \\
\hline 28 & 90 & 568,8 & 217,2 & 0,6 & 4,6 \\
\hline 29 & 90 & 469,1 & 0,0 & 0,2 & 0,7 \\
\hline
\end{tabular}

\begin{tabular}{|c|c|c|c|c|c|}
\hline ID & $\begin{array}{c}\text { Diám. } \\
(\mathrm{mm})\end{array}$ & $\begin{array}{c}\text { Longitud } \\
(\mathrm{m})\end{array}$ & $\begin{array}{c}\text { Caudal } \\
(\mathrm{I} / \mathrm{min})\end{array}$ & $\begin{array}{c}\mathrm{V} . \\
(\mathrm{m} / \mathrm{s})\end{array}$ & $\begin{array}{c}\text { Pérdidas } \\
\text { unitarias } \\
(\mathrm{m} / \mathrm{km})\end{array}$ \\
\hline 50 & 90 & 496 & 81,6 & 0,2 & 0,8 \\
\hline 51 & 200 & 593,2 & 544,8 & 0,3 & 0,5 \\
\hline 52 & 50 & 490,9 & 59,4 & 0,5 & 7,6 \\
\hline 53 & 150 & 1652,9 & 129,6 & 0,1 & 0,2 \\
\hline 54 & 200 & 1608,8 & 283,2 & 0,2 & 0,2 \\
\hline 55 & 90 & 1606,6 & 36,6 & 0,1 & 0,2 \\
\hline 56 & 50 & 1860 & 10,8 & 0,1 & 0,4 \\
\hline 57 & 50 & 1843,8 & 20,4 & 0,2 & 1,1 \\
\hline 58 & 150 & 1841,2 & 358,2 & 0,3 & 0,9 \\
\hline
\end{tabular}

Tabla 3.15 Características físicas y valores de operación normal para variables de nudos

\begin{tabular}{|c|c|c|}
\hline ID & $\begin{array}{c}\text { Demanda } \\
(\mathrm{I} / \mathrm{min})\end{array}$ & $\begin{array}{c}\text { Presión } \\
\text { (mca) }\end{array}$ \\
\hline $\mathrm{J} 1$ & 60 & 41,84 \\
\hline $\mathrm{J} 2$ & 60 & 39,89 \\
\hline $\mathrm{J} 3$ & 60 & 38,43 \\
\hline $\mathrm{J} 4$ & 60 & 36,84 \\
\hline $\mathrm{J} 5$ & 60 & 36,03 \\
\hline $\mathrm{J} 6$ & 60 & 35,80 \\
\hline $\mathrm{J} 7$ & 60 & 35,73 \\
\hline $\mathrm{J} 8$ & 60 & 35,93 \\
\hline $\mathrm{J} 9$ & 60 & 38,82 \\
\hline $\mathrm{J} 10$ & 60 & 40,06 \\
\hline $\mathrm{J} 11$ & 60 & 41,43 \\
\hline $\mathrm{J} 12$ & 60 & 43,10 \\
\hline $\mathrm{J} 13$ & 60 & 39,56 \\
\hline
\end{tabular}

\begin{tabular}{|c|c|c|}
\hline ID & $\begin{array}{c}\text { Demanda } \\
(I / \text { min })\end{array}$ & $\begin{array}{c}\text { Presión } \\
\text { (mca) }\end{array}$ \\
\hline J14 & 60 & 39,24 \\
\hline J15 & 60 & 43,62 \\
\hline J16 & 60 & 40,51 \\
\hline J17 & 180 & 37,01 \\
\hline J18 & 180 & 36,80 \\
\hline J19 & 180 & 38,90 \\
\hline J20 & 180 & 39,56 \\
\hline J21 & 60 & 38,93 \\
\hline J22 & 180 & 36,91 \\
\hline J23 & 180 & 36,62 \\
\hline J24 & 180 & 36,67 \\
\hline J25 & 180 & 37,27 \\
\hline J26 & 180 & 35,72 \\
\hline
\end{tabular}

\begin{tabular}{|c|c|c|}
\hline ID & $\begin{array}{c}\text { Demanda } \\
(1 / \text { min })\end{array}$ & $\begin{array}{c}\text { Presión } \\
(\text { mca })\end{array}$ \\
\hline J27 & 60 & 31,99 \\
\hline J28 & 180 & 36,99 \\
\hline J29 & 180 & 35,66 \\
\hline J30 & 60 & 31,99 \\
\hline J31 & 180 & 33,71 \\
\hline J32 & 180 & 35,90 \\
\hline J33 & 180 & 36,76 \\
\hline J34 & 180 & 38,42 \\
\hline J35 & 180 & 34,15 \\
\hline J36 & 180 & 33,29 \\
\hline J37 & 180 & 42,41 \\
\hline J38 & 180 & 47,07 \\
\hline
\end{tabular}

Una vez definido el caso, es necesario concretar las condiciones de contorno que regirán la delimitación de los sectores. En este caso, es necesario definir dos sectores, centrados en los nudos $\mathrm{J} 6$ y J36, una longitud mínima de tuberías englobadas en cada sector de 1.000 metros y una presión mínima de servicio de 20 mca.

Con todo esto, se procede a aplicar el algoritmo de cálculo teniendo como vértices los nudos J6 y J36, elegidos de manera aleatoria. Como resultado se obtienen todos los nudos englobados en dos zonas (Figura 3.13 a). Cada una de estas zonas se considerará una primera delimitación de los sectores finales. Nótese que muchas de las tuberías quedan englobadas en alguna de las dos zonas al estar en dichas zonas su nudos iniciales y finales, pero existen ciertas tuberías cuyos extremos se encuentran en zonas diferentes. Por ello, estas tuberías no quedan incluidas en ninguna zona.

Una vez predefinidos los sectores, el siguiente paso consiste en analizar qué tuberías pueden ser cerradas para aislar entre sí los sectores. Para ello, se empezará analizando las tuberías 
que no han quedado englobadas en ninguna zona. En estas tuberías o bien se deberán instalar válvulas de corte para aislar los sectores, o bien se deberán dejar abiertas para suministrar al segundo sector. En este caso, se dejarán abiertas las dos tuberías arteriales de 200 (nudos 9-10 y 20-21) para poder cumplir los requisitos de presión mínima. Nótese que, para revisar el comportamiento hidráulico del nuevo escenario, es necesaria la simulación hidráulica del mismo. La sectorización final se muestra en la Figura 3.13 b.
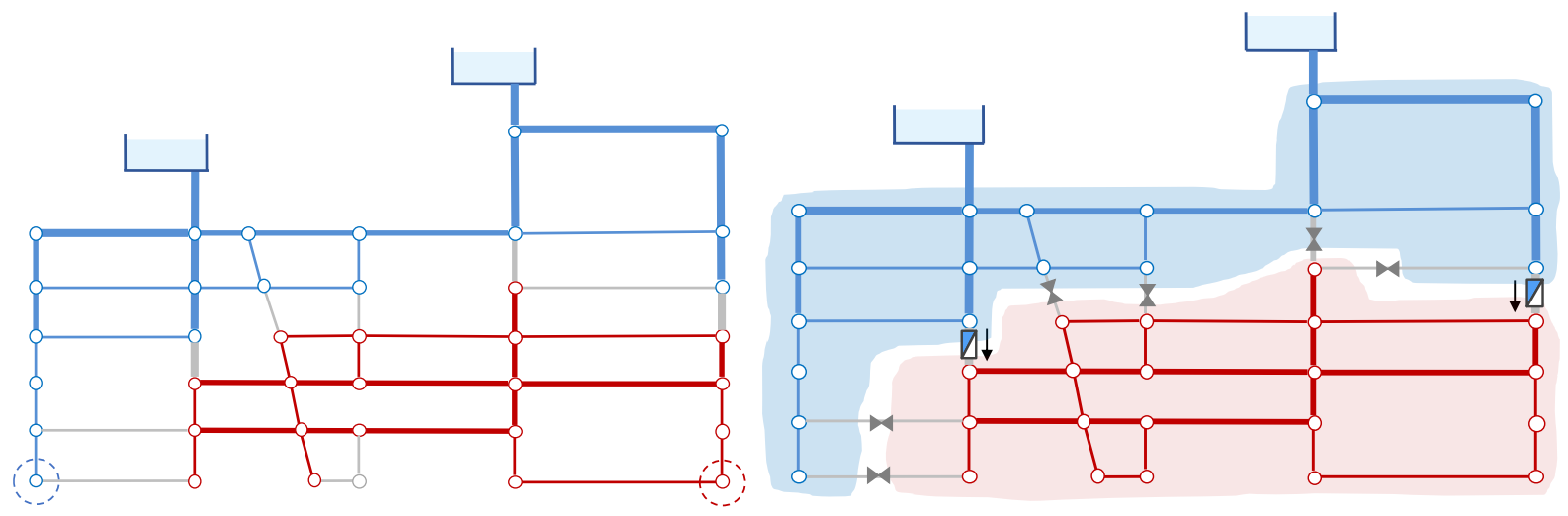

Figura 3.13. Delimitación de sectores tras el proceso de búsqueda (a) y sectorización final (b)

Una vez sectorizada la red, se puede comprobar (Tabla 3.16) cómo la presión media de la red disminuye en un 6,63\%, pasando de un valor medio de 37,88 mca a 35,37 mca. Evidentemente, al cerrar tuberías para aislar los sectores se está forzando al sistema a tener mayores pérdidas de carga. Si se analiza cómo se está usando la energía (Tabla 3.17), se puede comprobar cómo la energía perdida por fricción es mayor. Este hecho, que por lo general hace que la presión media de toda la red también disminuya, no afecta de igual modo en todas las zonas. En esta línea, se puede observar como la disminución de presión en el sector 1 ha sido de $0,85 \%$ frente al 12,44\% registrada en el segundo sector. Es importante destacar que, al modificar la conectividad de la red puede darse el caso de obtener aumentos en la presión media en determinados sectores tras la sectorización.

Tabla 3.16 Balance hídrico de la red tras la sectorización

\begin{tabular}{|l|c|c|c|c|}
\hline & Red actual & $\begin{array}{c}\text { Red } \\
\text { sectorizada }\end{array}$ & Sector 1 & Sector 2 \\
\hline Caudal suministrado (m²/día) & $6.566,40$ & $6.566,40$ & $2.419,20$ & $4.147,20$ \\
\hline Presión media antes de sectorizar (mca) & 37,88 & 37,88 & 39,96 & 36,02 \\
\hline Presión media tras sectorizar (mca) & - & 35,37 & 39,62 & 31,54 \\
\hline
\end{tabular}

Desde el punto de vista energético, como las presiones en el sector son menores, se reduce la energía entregada al usuario final (Tabla 3.17). En cuanto al análisis de la red global, antes y después de la sectorización, se puede observar cómo el valor de la eficiencia energética dinámica aumenta un $8,60 \%$, la energía perdida por fricción aumenta en un $24,05 \%$ y la energía entregada a los usuarios disminuye en un 7,91\%. 
Tabla 3.17 Balance energético de la red tras la sectorización

\begin{tabular}{|c|c|c|c|c|c|c|c|c|}
\hline \multirow{2}{*}{ RED } & \multirow{2}{*}{$\begin{array}{c}\text { Demanda } \\
\left(\mathrm{m}^{3} / \mathrm{d}\right)\end{array}$} & \multicolumn{4}{|c|}{ TOTA ENERGÍA PERDIDA (kWh/d) } & \multirow{2}{*}{$\begin{array}{c}\text { Energía } \\
\text { por caudal } \\
\text { unitario } \\
\left(\mathrm{kWh} / \mathrm{m}^{3}\right)\end{array}$} & \multirow{2}{*}{$\begin{array}{l}\text { Mín. } \\
\text { energía en } \\
\text { grifo de } \\
\text { consumidor }\end{array}$} & \multirow{2}{*}{$\begin{array}{c}\text { Ef. } \\
\text { energética } \\
(\%)\end{array}$} \\
\hline & & Fricción & Control & $\begin{array}{c}\text { Grifo } \\
\text { consumidor }\end{array}$ & Total & & & \\
\hline Red actual & $6.566,40$ & 221,52 & - & 673,2 & 894,72 & 0,10 & 357,84 & 53,16 \\
\hline $\begin{array}{l}\text { Red } \\
\text { Sectorizada }\end{array}$ & $6,566,40$ & 274,80 & - & 619,92 & 894,72 & 0,09 & 357,84 & 57,73 \\
\hline Sector 1 & $2.419,20$ & 205,92 & - & 264,24 & 470,16 & 0,11 & 131,76 & 49,89 \\
\hline Sector 2 & $4.147,20$ & 68,88 & - & 355,68 & 424,56 & 0,09 & 226,08 & 63,56 \\
\hline
\end{tabular}

En el caso anterior, se había supuesto un rendimiento del 100\%, por lo que se había supuesto una red sin fugas. Este escenario, además de ser improbable, no representa la realidad. Por ello, a continuación, se dotará a los nudos de consumo de unos caudales de fuga para simular el efecto que la sectorización tiene sobre estos, bajo un escenario con un rendimiento hidráulico del $85 \%$.

Los caudales de fuga se determinan admitiendo que son proporcionales tanto a la presión en su nudo correspondiente como a la longitud de las tuberías que en él convergen (hipótesis de distribución uniforme). Cada fuga nodal se caracteriza con su correspondiente coeficiente del emisor cuyos valores se determinan mediante según el proceso descrito en Almandoz et al. (2005). Dichos coeficientes para cada nudo son los siguientes:

Tabla 3.18 Coeficientes emisores nodales

\begin{tabular}{|c|c|}
\hline $\mathrm{ID}$ & Emisor $\left(\mathrm{m}^{3-a} \mathrm{~s}\right)$ \\
\hline $\mathrm{J} 1$ & 0,036916 \\
\hline $\mathrm{J} 2$ & 0,044588 \\
\hline $\mathrm{J} 3$ & 0,043577 \\
\hline $\mathrm{J} 4$ & 0,015306 \\
\hline $\mathrm{J} 5$ & 0,042060 \\
\hline $\mathrm{J} 6$ & 0,033845 \\
\hline $\mathrm{J} 7$ & 0,034470 \\
\hline $\mathrm{J} 8$ & 0,059295 \\
\hline $\mathrm{J} 9$ & 0,032470 \\
\hline $\mathrm{J} 10$ & 0,044513 \\
\hline $\mathrm{J} 11$ & 0,058717 \\
\hline $\mathrm{J} 12$ & 0,064849 \\
\hline $\mathrm{J} 13$ & 0,036472 \\
\hline
\end{tabular}

\begin{tabular}{|c|c|}
\hline ID & Emisor $\left(\mathrm{m}^{3-\alpha} \mathrm{s}\right)$ \\
\hline J14 & 0,053175 \\
\hline J15 & 0,088291 \\
\hline J16 & 0,057850 \\
\hline J17 & 0,042827 \\
\hline J18 & 0,028720 \\
\hline J19 & 0,047976 \\
\hline J20 & 0,048566 \\
\hline J21 & 0,047093 \\
\hline J22 & 0,072001 \\
\hline J23 & 0,053203 \\
\hline J24 & 0,027238 \\
\hline J25 & 0,041661 \\
\hline J26 & 0,039654 \\
\hline
\end{tabular}

\begin{tabular}{|c|c|}
\hline ID & Emisor $\left(\mathrm{m}^{3-\alpha} \mathrm{s}\right)$ \\
\hline J27 & 0,014593 \\
\hline J28 & 0,044261 \\
\hline J29 & 0,042546 \\
\hline J30 & 0,014890 \\
\hline J31 & 0,035986 \\
\hline J32 & 0,041731 \\
\hline J33 & 0,071048 \\
\hline J34 & 0,046430 \\
\hline J35 & 0,014364 \\
\hline J36 & 0,035441 \\
\hline J37 & 0,049628 \\
\hline J38 & 0,060221 \\
\hline
\end{tabular}

Así, el caudal de fuga nodal se calculará siguiendo a Cobacho et al. (2014), según la siguiente ecuación:

$$
q_{l i}\left(t_{k}\right)=C_{E} \cdot \Delta H^{\propto}
$$

donde $C_{E}\left(m^{3-\propto} / \mathrm{s}\right)$ es el coeficiente del emisor asignado a cada nudo del sistema, $\Delta H(\mathrm{~m})$ es el salto de presiones que experimenta el agua al atravesar el orificio de fuga y $\alpha=0,5$ es el 
exponente de emisor que representa las características propias del material de la tubería y que adopta valores diferentes según el tipo de material de las tuberías.

Una vez sectorizada la red bajo este nuevo escenario, se puede comprobar (Tabla 3.19) cómo los resultados obtenidos están en línea como los obtenidos en el escenario sin fugas. Respecto al volumen incontrolado fugado, este ha disminuido en un 4,53\%. Evidentemente, al cerrar tuberías para aislar los sectores se está forzando al sistema a tener mayores pérdidas de carga, y ello se refleja en una pérdida de presión media.

Tabla 3.19 Balance hídrico de la red tras la sectorización

\begin{tabular}{|c|c|c|c|c|}
\hline & Red actual & $\begin{array}{c}\text { Red } \\
\text { sectorizada }\end{array}$ & Sector 1 & Sector 2 \\
\hline Caudal suministrado antes de sectorizar (m³/día) & $7.648,08$ & $7.648,08$ & $2.952,29$ & $4.695,79$ \\
\hline Caudal fugado antes de sectorizar (m³/día) & 849,72 & 849,72 & 449,12 & 400,59 \\
\hline Presión media antes de sectorizar (mca) & 34,06 & 34,06 & 36,62 & 31,75 \\
\hline Caudal suministrado después de sectorizar ( $\mathrm{m}^{3} /$ día) & - & $7.608,68$ & $2.951,76$ & $4.656,68$ \\
\hline Caudal fugado después de sectorizar (m³/día) & - & 811,21 & 447,06 & 364,16 \\
\hline Presión media tras sectorizar (mca) & - & 31,02 & 36,15 & 26,4 \\
\hline Caudal fugado mínimo (m³/día) & 643,91 & 643,91 & 328,48 & 315,43 \\
\hline
\end{tabular}

Desde el punto de vista energético, como las presiones en el sector son menores, se reduce la energía entregada al usuario final (Tabla 3.20). En cuanto al análisis de la red global, antes y después de la sectorización, se puede observar cómo el valor de la eficiencia energética dinámica aumenta un $11,32 \%$, la energía perdida por fricción lo hace en un $20,86 \%$ y la energía entregada a los usuarios disminuye en un $11,89 \%$.

Tabla 3.20 Balance energético de la red con fugas tras la sectorización

\begin{tabular}{|c|c|c|c|c|c|c|c|c|}
\hline \multirow[b]{2}{*}{ RED } & \multirow[b]{2}{*}{$\begin{array}{l}\text { Demanda } \\
\qquad\left(\mathrm{m}^{3} / \mathrm{d}\right)\end{array}$} & \multicolumn{4}{|c|}{ TOTA ENERGÍA PERDIDA (kWh/d) } & \multirow{2}{*}{$\begin{array}{c}\text { Energía } \\
\text { por } \\
\text { caudal } \\
\text { unitario } \\
\left(\mathrm{kWh} / \mathrm{m}^{3}\right)\end{array}$} & \multirow{2}{*}{$\begin{array}{l}\text { Mín. } \\
\text { energía en } \\
\text { grifo de } \\
\text { consumidor }\end{array}$} & \multirow{2}{*}{$\begin{array}{c}\text { Ef. } \\
\text { energética } \\
\text { dinámica } \\
(\%)\end{array}$} \\
\hline & & Fricción & Control & $\begin{array}{c}\text { Grifo } \\
\text { consumidor }\end{array}$ & Total & & & \\
\hline Red actual & $7.648,08$ & 334,80 & - & 707,04 & $1.042,08$ & 0,09 & 416,88 & 58,95 \\
\hline $\begin{array}{l}\text { Red } \\
\text { Sectorizada }\end{array}$ & $7.609,68$ & 404,88 & - & 631,92 & $1.036,80$ & 0,08 & 414,72 & 65,62 \\
\hline Sector 1 & $2.951,76$ & 310,08 & - & 297,36 & 607,44 & 0,10 & 160,80 & 54,09 \\
\hline Sector 2 & $4.657,68$ & 94,80 & - & 334,56 & 429,36 & 0,07 & 253,92 & 75,87 \\
\hline
\end{tabular}

Si se realiza una comparativa entre ambos escenarios, se puede observar un comportamiento hidráulico (Tabla 3.16 y Tabla 3.19) y energético (Tabla 3.17 y Tabla 3.20) similar. En el escenario con fugas, se obtienen unos valores de energía de fricción y de grifo de consumidor mayores debido a que también lo son los caudales circulares por la red. Esto hace que las pérdidas por fricción sean mayores, con ello menor la presión media y, en definitiva, una eficiencia energética mayor tanto para el global de la red como para cada uno de los sectores.

\subsubsection{Diseño sin restricciones}

En el anterior apartado, se abordada el procedimiento de sectorización propuesto bajo ciertas restricciones. Donde, una vez ejecutado, se puede asegurar que los sectores obtenidos como 
resultado engloban a aquellos nudos que obtienen un valor mínimo de eficiencia energética dinámica para las condiciones de contorno fijadas. Es decir, se obtiene como resultado un mínimo local, no pudiendo asegurar que sea el mínimo global. Para poder asegurar el mínimo global, es necesario plantear un procedimiento de búsqueda entre todas las combinaciones de agrupación posibles y seleccionar aquella zona con un valor menor, sin considerar ningún factor de delimitación.

A continuación, se adjunta el diagrama de flujo de dicho procedimiento de diseño sin restricciones: 


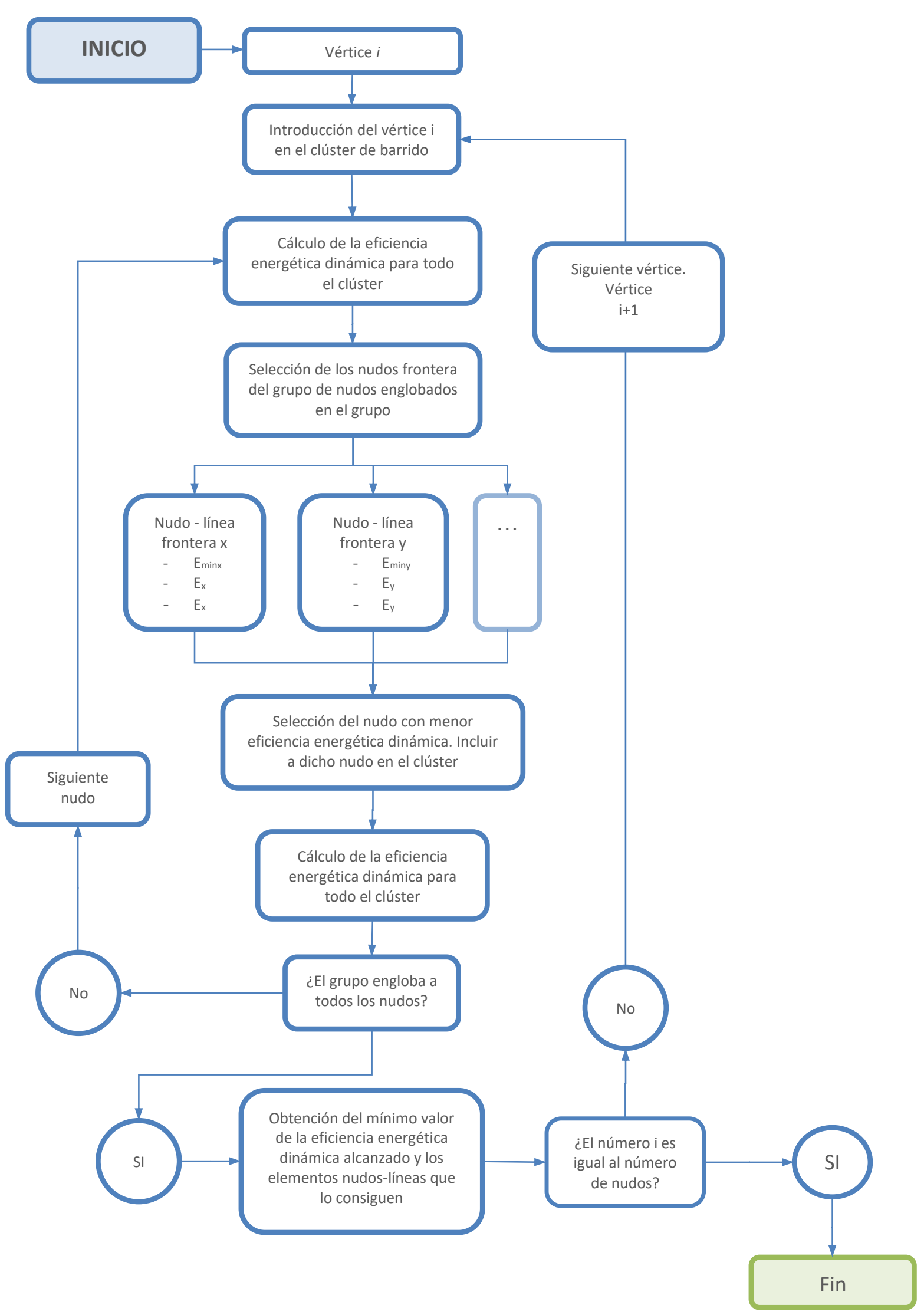

Figura 3.14. Diagrama de flujo del procedimiento de diseño sin restricciones 


\subsubsection{Ejemplo numérico}

Para una mejor compresión de la metodología sin restricciones, esta se va a aplicar sobre la misma red que en el caso anterior (Figura 3.11). Como se ha descrito, se plantea la sectorización sin imponer ningún tipo de condiciones externas que limiten el diseño. Aun así, y para evitar sectores demasiado pequeños, es decir, que la eficiencia energética dinámica menor se produzca para un pequeño número de nudos, se limitará cada sector a que contenga un número superior al $10 \%$ del total.
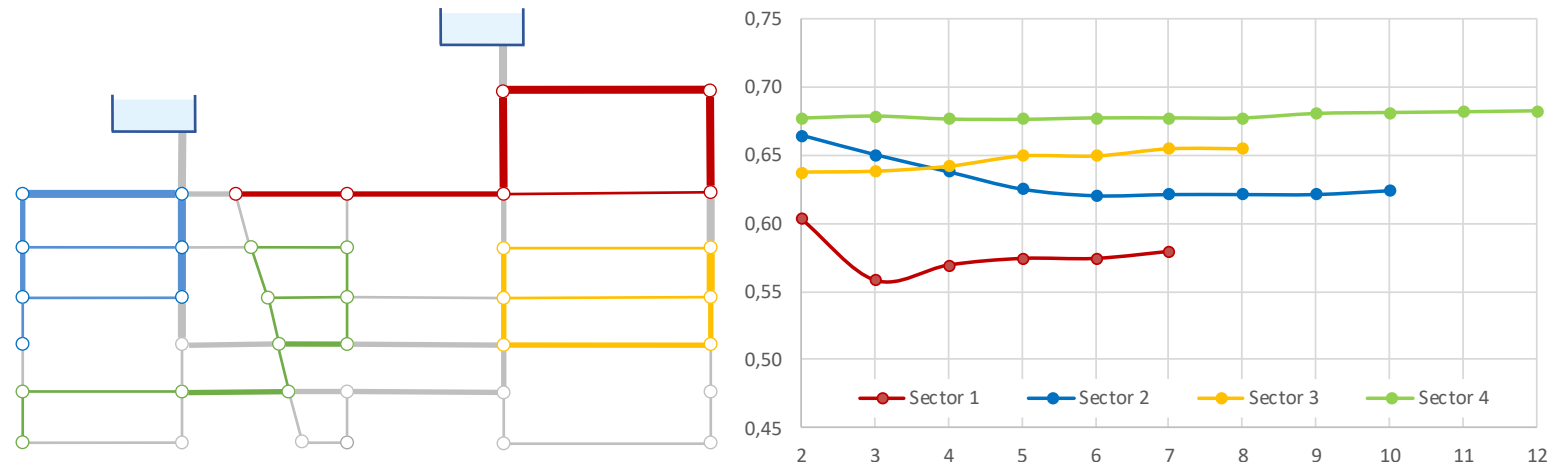

Figura 3.15. Delimitación de sectores tras el proceso de búsqueda sin restricciones (a) y variación de la eficiencia energética dinámica según el número de pasos y áreas (b)

Como resultado, se obtienen un total de cuatro zonas diferenciadas (Figura 3.15 a). En primer lugar, se obtiene una primera zona (roja) que tiene una eficiencia energética dinámica del $57,96 \%$. En segundo lugar, se destaca la segunda zona (azul) con un valor del $62,42 \%$. Acto seguido, la tercera zona (naranja) con una eficiencia del $65,51 \%$. Y en último lugar, se obtiene una zona (verde) que presenta un valor del 68,26\%.

En la Tabla 3.21 se muestran agrupados los nudos y tuberías que forman cada una de las zonas, en las que el número de paso muestra el orden de entrada de los elementos al grupo.

Tabla 3.21 Agrupación de los nudos y líneas según zonas

\begin{tabular}{|c|c|c|c|c|c|c|c|c|c|c|c|c|}
\hline \multirow{2}{*}{$\begin{array}{c}\text { № } \\
\text { Paso }\end{array}$} & \multicolumn{3}{|c|}{ Zona 1 (rojo) } & \multicolumn{3}{|c|}{ Zona 2 (azul) } & \multicolumn{3}{|c|}{ Zona 3 (naranja) } & \multicolumn{3}{|c|}{ Zona 4 (verde) } \\
\hline & $\begin{array}{l}\text { ID } \\
\text { nudo }\end{array}$ & $\begin{array}{l}\text { ID } \\
\text { línea }\end{array}$ & $\begin{array}{c}\text { Ef. } \\
\text { Energ. }\end{array}$ & $\begin{array}{c}\text { ID } \\
\text { nudo }\end{array}$ & $\begin{array}{l}\text { ID } \\
\text { línea }\end{array}$ & $\begin{array}{c}\text { Ef. } \\
\text { Energ. }\end{array}$ & $\begin{array}{c}\text { ID } \\
\text { nudo }\end{array}$ & $\begin{array}{l}\text { ID } \\
\text { línea }\end{array}$ & $\begin{array}{c}\text { Ef. } \\
\text { Energ. }\end{array}$ & $\begin{array}{c}\text { ID } \\
\text { nudo }\end{array}$ & $\begin{array}{l}\text { ID } \\
\text { línea }\end{array}$ & $\begin{array}{c}\text { Ef. } \\
\text { Energ. }\end{array}$ \\
\hline 1 & 14 & - & - & 4 & - & - & 19 & - & - & 17 & - & - \\
\hline 2 & 15 & 4 & $60,34 \%$ & 3 & 22 & $66,43 \%$ & 20 & 54 & $63,73 \%$ & 18 & 45 & $67,75 \%$ \\
\hline 3 & 38 & 8 & $55,79 \%$ & 10 & 33 & $65,03 \%$ & 21 & 18 & $63,80 \%$ & 24 & 27 & $67,89 \%$ \\
\hline 4 & 37 & 6 & $56,93 \%$ & 11 & 35 & $63,79 \%$ & 34 & 17 & $64,18 \%$ & 25 & 28 & $67,68 \%$ \\
\hline 5 & 16 & 5 & $57,43 \%$ & 12 & 34 & $62,55 \%$ & 22 & 10 & $64,96 \%$ & 28 & 49 & $67,66 \%$ \\
\hline 6 & - & 7 & $57,43 \%$ & 1 & 1 & $62,06 \%$ & - & 55 & $64,96 \%$ & 23 & 47 & $67,76 \%$ \\
\hline 7 & 13 & 3 & $57,96 \%$ & 2 & 20 & $62,15 \%$ & 33 & 11 & $65,51 \%$ & - & 44 & $67,76 \%$ \\
\hline 8 & & & & - & 21 & $62,15 \%$ & & 56 & $65,51 \%$ & - & 48 & $67,76 \%$ \\
\hline 9 & & & & - & 31 & $62,15 \%$ & & & & 26 & 29 & $68,07 \%$ \\
\hline 10 & & & & 9 & 36 & $62,42 \%$ & & & & 8 & 39 & $68,14 \%$ \\
\hline 11 & & & & & & & & & & 5 & 42 & $68,19 \%$ \\
\hline 12 & & & & & & & & & & 6 & 24 & $68,26 \%$ \\
\hline
\end{tabular}


Al igual que pasaba con el anterior caso de estudio, como resultado del algoritmo de búsqueda no se obtienen los sectores definitivos, sino una delimitación de estos (Figura 3.15 a). Por ello, es necesario analizar qué tuberías se deberán dejar abiertas y cuáles se deberán de cerrar, así como colocar contadores de control del agua inyectada en cada sector (Figura 3.16).

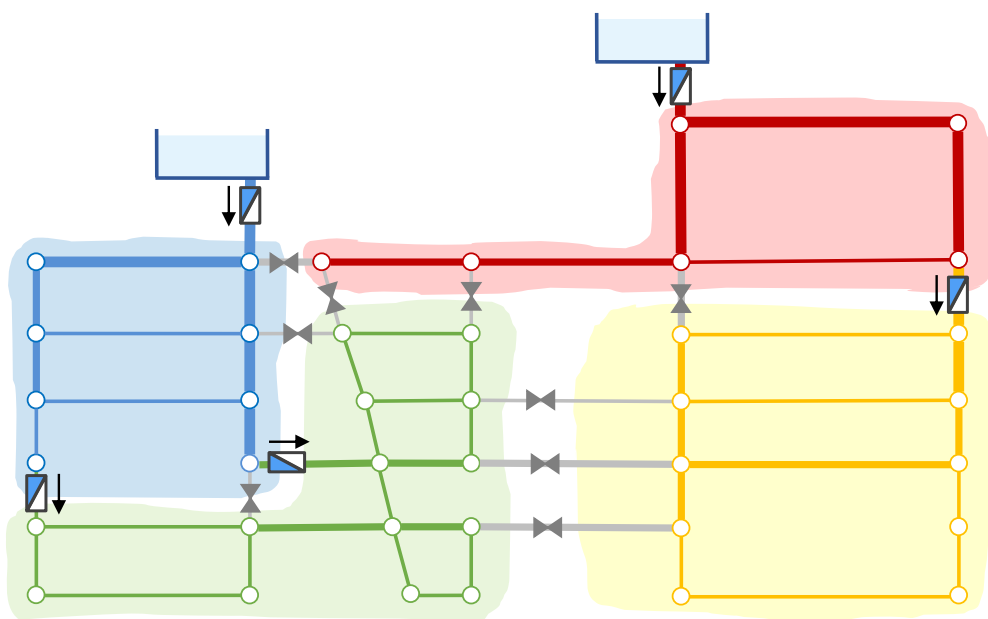

Figura 3.16. Sectorización final propuesta

Analizando los resultados obtenidos para cada escenario (Tabla 3.22), el primer aspecto a considerar es que el nivel de presiones medio de la red tras sectorizar ha descendido un $17,63 \%$. Evidentemente, al cerrar más tuberías se está forzando una mayor pérdida de carga en aquellas tuberías que quedan abiertas. Cabe destacar que, aunque la presión media desciende en los sectores 2, 3 y 4, no lo hace en el sector 1. Esto es debido a que el cierre de tuberías ha cambiado el modo de regulación del sistema, haciendo que el depósito J39 aporte menos caudal y se produzcan menos pérdidas de carga en la tubería de alimentación.

Tabla 3.22 Balance hídrico de la red tras la sectorización

\begin{tabular}{|l|c|c|c|c|c|c|}
\hline & $\begin{array}{c}\text { Red } \\
\text { actual }\end{array}$ & $\begin{array}{c}\text { Red } \\
\text { sectorizada }\end{array}$ & Sector 1 & Sector 2 & Sector 3 & Sector 4 \\
\hline Caudal suministrado (m²/ía) & $6.566,40$ & $6.566,40$ & 864,00 & 691,20 & $2.419,20$ & $2.592,00$ \\
\hline Presión media antes de sectorizar (mca) & 37,88 & 37,88 & 42,07 & 40,05 & 36,65 & 35,73 \\
\hline Presión media tras sectorizar (mca) & - & 31,20 & 42,75 & 38,67 & 24,80 & 26,55 \\
\hline
\end{tabular}

Si se estudian ahora los diferentes valores de energías consumidas y perdidas en la red, así como el valor de la eficiencia energética dinámica para cada uno de los sectores obtenidos, se observa un comportamiento similar al analizado en el estudio del diseño con restricciones, aunque es importante destacar que los valores de las eficiencias de los cuatro sectores presentan unos valores mayores a los obtenidos por el algoritmo para las cuatro zonas delimitadas. Esto es debido a la ejecución de la sectorización, la cual ha implicado el cierre de una serie de tuberías para aislar los sectores, y esto ha modificado a la baja los valores de presiones en toda la red. También hay que notar que el proceso de cálculo del algoritmo puede dejar ciertos nudos y líneas fuera de alguno de los sectores por no cumplir la condición impuesta de longitud mínima. Posteriormente, estos nudos y líneas son englobados dentro de alguno de los sectores modificando su eficiencia energética. La eficiencia energética dinámica es mayor en el sector 3 y no en el 4, como se había definido en la agrupación de zonas. 
Tabla 3.23 Balance energético de la red tras la sectorización

\begin{tabular}{|c|c|c|c|c|c|c|c|c|}
\hline \multirow[b]{2}{*}{ RED } & \multirow{2}{*}{$\begin{array}{l}\text { Demanda } \\
\qquad\left(m^{3} / d\right)\end{array}$} & \multicolumn{4}{|c|}{ TOTA ENERGÍA PERDIDA (kWh/d) } & \multirow{2}{*}{$\begin{array}{c}\text { Energía por } \\
\text { caudal } \\
\text { unitario } \\
\left(\mathrm{kWh} / \mathrm{m}^{3}\right)\end{array}$} & \multirow{2}{*}{$\begin{array}{l}\text { Mín. } \\
\text { energía en } \\
\text { grifo de } \\
\text { consumidor }\end{array}$} & \multirow{2}{*}{$\begin{array}{c}\text { Ef. } \\
\text { Energética } \\
\text { dinámica } \\
(\%)\end{array}$} \\
\hline & & Fricción & Control & $\begin{array}{c}\text { Grifo } \\
\text { consumidor }\end{array}$ & Total & & & \\
\hline Red actual & $6.566,40$ & 221,52 & - & 673,20 & 894,72 & 0,1 & 357,84 & 53,16 \\
\hline $\begin{array}{l}\text { Red } \\
\text { sectorizada }\end{array}$ & $6.566,40$ & 368,64 & - & 526,08 & 894,72 & 0,08 & 357,84 & 68,02 \\
\hline Sector 1 & 864,00 & 119,52 & - & 100,56 & 220,08 & 0,12 & 47,04 & 46,82 \\
\hline Sector 2 & 691,20 & 115,44 & - & 72,96 & 188,16 & 0,11 & 37,68 & 51,71 \\
\hline Sector 3 & $2.419,20$ & 66,72 & - & 160,56 & 227,28 & 0,07 & 131,76 & 82,15 \\
\hline Sector 4 & $2.592,00$ & 66,96 & - & 192,24 & 259,20 & 0,07 & 141,36 & 73,51 \\
\hline
\end{tabular}




\section{Capítulo 4 \\ Sensores para la localización de fugas}

\subsection{Introducción}

A la hora de dar una definición universal del término sensor no existe un acuerdo general, e incluso hay ciertas discrepancias entre diferentes autores y épocas. Así, en los años 90, Conde (1996) definía el sensor como un "Dispositivo capaz de registrar de forma directa, continua y reversible un parámetro físico (sensor físico) o la concentración de una especie química (sensor químico)", donde se resaltaba la importancia de proporcionar lecturas en tiempo real. Años después, Ramírez et al. (2014) lo definen como un "Dispositivo de entrada que provee una salida manipulable de la variable física medida". Pero si en algo coindicen todos los autores es el papel tan importante que los sensores han desarrollado, están desarrollando y desarrollarán en todos los aspectos de la sociedad. Tanto es así que, en febrero de 2003, el MIT identificó las redes de sensores inalámbricos como una de las diez tecnologías emergentes que cambiarían el mundo (Huang, 2003). Incluso, algunos autores (Chong \& Kumar, 2003) se aventuran a etiquetar a las redes inalámbricas de sensores como una de las tecnologías más importantes del siglo XXI.

Y no es para menos. La versatilidad de los sensores es tal que permite la monitorización de cualquier propiedad, recurriendo para ello a una tecnología u otra. Así, la aplicación de los sensores está presente en todos los ámbitos de la sociedad, tales como la automatización de edificios, domótica, medicina, control industrial o agricultura, por citar solo algunos ejemplos.

En los sistemas de distribución de agua, los sensores también están presentes desde hace años y de una manera muy generalizada. El control del nivel de la lámina de agua en los depósitos, la lectura en continuo del caudal trasegado o la medición de la concentración de cloro en la red de distribución, son algunas de las aplicaciones más frecuentes usadas por los gestores de estos sistemas. Sin embargo, ha sido recientemente, e impulsada por el desarrollo de estas tecnologías, cuando la aplicación de los sensores ha dado un enorme paso hacía la 
optimización de procesos. Lai et al. (2010) desarrollan el uso de sensores que, introducidos en el interior de las tuberías y arrastrados por el propio flujo del agua, son capaces de dibujar el trazado de estas. Gong et al. (2016) emplean el uso de pequeños sensores que, al igual que los propuestos por los anteriores autores, se mueven libremente por las tuberías. Pero esos equipos se encargan de recoger datos sobre posibles escapes incontrolados o sobre la calidad del agua (Wu et al., 2014). Estudios más recientes, emplean sensores de fibra óptica instalados sobre la pared interior de las tuberías para monitorizar la presión en continuo (Wong et al., 2018).

Profundizando en los sistemas de distribución de agua, y recurriendo nuevamente al problema de las fugas, los sensores también están presentes en la detección y localización de estas. Aunque estos métodos existen desde hace años, es ahora cuando están sufriendo un fuerte desarrollo gracias a la tecnología actual. Para grandes tuberías arteriales, se emplean los sensores para medir la presión y, en algunos casos se pueden obtener datos que incluso se emplean para analizar hidráulicamente el transitorio y localizar fugas existentes (Puust et al., 2010). La limitación que presentan estas metodologías es que requieren grandes caudales, con lo que se convierten en inoperativas para pequeñas redes de distribución.

Para redes malladas de distribución, la bibliografía propone métodos de localización de las fugas a partir de medidas de presión y de un análisis de sensibilidad hidráulica (variabilidad). Es decir, se aprovecha la interdependencia de todos los parámetros hidráulicos para una adecuada localización. Pudar \& Liggett (1992) consideran la dependencia entre el volumen fugado, la presión de la red y la sección del orificio de fuga. Más recientemente, la mayoría de los estudios se han centrado en determinar la ubicación de los sensores para detectar un mayor número de fallos (Blesa et al., 2016; Casillas et al., 2015; Gamboa-Medina \& Reis, 2017; Sarrate et al., 2014). El resultado, en todo caso, depende del número de sensores instalados (Xie et al., 2017), y está limitado por el presupuesto y la estrategia seguida (FuchsHanusch \& Steffelbauer, 2017). A partir de las medidas de los sensores de presión, Pérez et al. (2011) proponen analizar la diferencia entre dichos datos y sus equivalentes proporcionados por el modelo de simulación, y ante una discrepancia, hacen uso de la matriz de sensibilidad de fuga (leak sensitivity matrix) para plantear su localización. En condiciones ideales, todas estas metodologías presentan grandes resultados, pero la calidad de éstos decrece en presencia de errores tanto en las demandas como en las mediciones. Para intentar minimizar este efecto, estudios posteriores (Casillas et al., 2014) introducen en sus cálculos un análisis en periodo extendido de las mediciones. Otros autores proponen desarrollos similares basados en análisis de sensibilidad para la búsqueda de fugas (Gamboa-Medina \& Reis, 2017; Möderl et al., 2011; Steffelbauer et al., 2014), pero todos requieren plantear numerosos escenarios, y sus simulaciones correspondientes, para explorar los cambios obtenidos. Por ello y considerando el funcionamiento dinámico de las redes de distribución y la siempre imprevisible aparición de las fugas, el tiempo necesario para el desarrollo de dichos análisis puede limitar su uso práctico.

En línea con las referencias apuntadas, y partiendo de los logros conseguidos con la sectorización de las redes frente a los volúmenes incontrolados fugados, el presente capítulo plantea una técnica no intrusiva alternativa que logre los mismos resultados, pero sin afectar a la capacidad hidráulica de la red y a la calidad del agua. Así, se presenta una metodología para la localización de fugas basada en un nuevo cálculo simplificado de la sensibilidad de los elementos de la red y apoyado en la monitorización de las redes (Salguero et al., 2018). De este modo, combina, por una parte, el conocimiento del comportamiento medio específico de cada elemento ante un incremento de demanda en cualquier punto de la red (sensibilidad), y 
por otra, la información proporcionada por los sensores de presión y caudal. El fin de todo ello es llegar a una formulación matricial de las ecuaciones del comportamiento de la red en la que las incógnitas son, directamente, los caudales de fuga en cada nudo, y que se puede resolver por el clásico procedimiento del gradiente (Todini \& Pilati, 1987), obteniendo como resultado dónde se está produciendo el volumen incontrolado.

\subsection{Ecuaciones básicas de funcionamiento}

Por lo general, las redes de distribución de agua potable son grandes redes malladas con elementos de producción, transporte, almacenamiento y regulación. En el diseño y análisis de estas redes entran en juego gran cantidad de variables e incógnitas y unos sistemas de ecuaciones no lineales cuya resolución se requiere.

Dada una red de agua con $p$ tuberías, $n$ nodos y $m$ puntos de suministro, el método para modelar su comportamiento hidráulico a través de ecuaciones de conservación ya ha sido establecido correctamente (Todini \& Pilati, 1987; Todini \& Rossman, 2013). La primera ecuación es la ecuación de conservación de la masa aplicada a cada nodo de red:

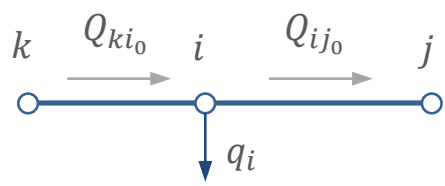

$$
\sum_{k=1}^{n_{i}} Q_{i k_{0}}+q_{i}=0
$$

donde $n_{i}$ es el número total de nudos conectados con el nudo $i, Q_{i k_{0}}$ es el caudal circulante por cada tubería que conecta cada nudo $k$ con el nudo $i$ y $q_{i}$ es la demanda del nudo $i$. Se establece con signo positivo el flujo que llega al nudo y negativo cuando este lo abandona.

La segunda ecuación es la ecuación de conservación de energía aplicada a cada tubería de la red:

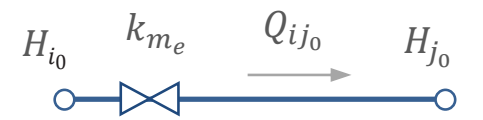

$$
H_{i_{0}}-H_{j_{0}}=h_{f_{i j_{0}}}=r_{i j} Q_{i j_{0}}\left|Q_{i j_{0}}\right|
$$

donde $H_{i_{0}}$ y $H_{j_{0}}$ son las alturas piezométricas de los nudos $i$ y $j$, respectivamente, $h_{f_{i j}}$ representa las pérdidas por fricción en la línea que conecta los nudos $i$ y $j$, y $r_{i j}$ y $Q_{i j_{0}}$ son, respectivamente, la resistencia hidráulica y el caudal circulante por la tubería que conecta los nudos $i$ y $j$.

Ambos conjuntos de ecuaciones de conservación de la masa y la energía, pueden estructurarse mediante notación matricial (Todini \& Pilati, 1987):

$$
\left[\begin{array}{ccc}
A_{11} & \vdots & A_{12} \\
\cdots & \cdots & \cdots \\
A_{21} & \vdots & 0
\end{array}\right] \cdot\left[\begin{array}{c}
Q \\
\cdots \\
H
\end{array}\right]=-\left[\begin{array}{c}
A_{10} \cdot H_{0} \\
q
\end{array}\right]
$$

donde $A_{11}$ es una matriz diagonal, de dimensión $p$ que representa las pérdidas por fricción de cada tubería; $A_{12}$ es la matriz de conectividad asociada a cada uno de los nudos de la red, de dimensiones $[p, n]$ y valores de celdas - 1 en la columna correspondiente al nudo inicial de la línea ij y 1 en la columna correspondiente al nudo final de la línea ij, o lo que es lo mismo, el valor $A_{12}(i, j)$ será - 1 si el flujo de la línea ij abandona el nudo $j$, 0 si no hay tal línea entre los 
nudos $i$ y $j$ o 1 si el flujo de la línea $i j$ entra al nudo $j$; la matriz $A_{21}$ corresponde con la matriz traspuesta de $A_{12}$; $Q$ es el vector de los caudales (desconocidos) de las $p$ líneas; $H$ es el vector de las alturas piezométricas (desconocidas) de los $n$ nudos; $H_{0}$ es el vector de las alturas piezométricas (conocidas) de los $m$ puntos de suministro; $A_{10}$ es el vector con los valores (conocidos) para los $m$ nudos de altura piezométrica fija, con dimensiones $[p, m]$ y con valores de -1 en las filas correspondientes a los tramos conectados a los nudos de altura piezométrica fija; y $q$ es el vector con las demandas nodales (conocidas).

La anterior matriz $A_{11}$, que recoge las pérdidas hidráulicas causadas por los elementos instalados en la red o la existencia de bombeos, tiene la siguiente estructura:

$$
\left[A_{11}\right]=\left[\begin{array}{cccc}
\alpha_{1} Q_{1}^{\left(n_{1}-1\right)}+\beta_{1}+\frac{\gamma_{1}}{Q_{1}} & 0 & \ldots & 0 \\
0 & \alpha_{2} Q_{2}^{\left(n_{2}-1\right)}+\beta_{2}+\frac{\gamma_{2}}{Q_{2}} & \ldots & 0 \\
\vdots & \vdots & \ddots & \vdots \\
0 & 0 & \ldots & \alpha_{N T} Q_{N T}^{\left(n_{N T}-1\right)}+\beta_{N T}+\frac{\gamma_{N T}}{Q_{N T}}
\end{array}\right]
$$

donde $n$ es un exponente que depende de la ecuación para obtener el cálculo de la fricción utilizada (en el caso de emplear la ecuación de Darcy-Weisbach, este exponente tendrá un valor de 2) y $\alpha, \beta$ y $\gamma$ corresponden con parámetros de caracterización de las tuberías, válvulas y bombas.

Para la resolución de las ecuaciones planteadas en 4.3, es decir, para obtener los valores de caudal en las líneas y las alturas piezométricas en los nudos, es necesaria la resolución de un sistema de ecauciones no lineales. Para ello se cuenta con varios métodos de análisis como el Método de Hardy-Cross (Cross, 1936) con corrección de caudales en los circuitos o alturas piezométrias en los nodos o el método de la teoría líneal (Wood \& Charles, 1972). Pero con el desarrollo del mundo digital fueron tomando protagonismo unos métodos sobre otros. Uno de ellos fue el método iterativo de Newton-Raphson desarrollado por los investigadores Martin \& Peters (1963), que consiste en un método numérico que permite el cálculo de las raices de las ecucaiones de forma rápida, mediante iteraciones sucesivas siguiendo la regla de Newton: Si $X_{0}$ es una aproximación a la raiz de $f(X)$, entonces $X_{0}+\partial X_{0}$ será una mejor aproximación, donde, y aplicando el teorema de Taylor para $\left(f(X)_{0}+\partial X_{0}\right)$ :

$$
\partial X_{0}=-\frac{f\left(X_{0}\right)}{f^{\prime}\left(X_{0}\right)}
$$

En el caso, como es el nuestro, de tener un sistema de $\mathrm{N}$ ecuaciones no lineales también se puede emplear este método para encontrar sus raíces, siguiendo la siguiente expresión:

$$
-f_{i}\left(X_{01}, X_{02} \ldots X_{0 N}\right)=\sum_{j=1}^{N} \frac{\partial f_{i}}{\partial X_{j}} \partial X_{0 j}
$$

Escrita en forma matricial, la anterior ecuación 4.6 quedaría expresada de la siguiente manera: 


$$
\left[\begin{array}{ccc}
\frac{\partial f_{1}}{\partial X_{1}} & \cdots & \frac{\partial f_{1}}{\partial X_{N}} \\
\vdots & \ddots & \vdots \\
\frac{\partial f_{N}}{\partial X_{1}} & \cdots & \frac{\partial f_{N}}{\partial X_{N}}
\end{array}\right] \cdot\left[\begin{array}{c}
\partial X_{01} \\
\vdots \\
\partial X_{0 N}
\end{array}\right]=\left[\begin{array}{c}
-f_{1} \\
\vdots \\
-f_{N}
\end{array}\right]
$$

Y de modo general y más compacto, el anterior sistema puede escribirse como:

$$
\begin{gathered}
{\left[\frac{D F}{D X}\right] \cdot[D X]=[-F]} \\
{[D X]=\left[\frac{D F}{D X}\right]^{-1} \cdot[-F]}
\end{gathered}
$$

En la actualidad, el método que emplean la mayoría del software de simulación hidráulica, liderado por Epanet (Rossman, 2002), es el conocido como método del gradiente. Este método, como bien recoge Saldarriaga (2011), fue desarrollado por Todini, O'Connell y Salgado durante los años 80 y planteado de manera definitiva por Todini \& Pilati (1987) años después. Y calcula de manera simultánea los caudales en las tuberías y las alturas piezométricas en los nudos combinando las ecuaciones de continuidad con las ecuaciones de energía para, posteriormente, linealizar las ecuaciones de energía utilizando una expansión en serie de Taylor. Como puede observarse, el sistema de ecuaciones 4.3 no puede ser resuelto de manera directa al tratarse de una ecuación no lineal. Por ello, este método realiza una expansión truncada de Taylor, obteniendo la siguiente estructura:

$$
\left[\begin{array}{cc}
n\left[A_{11}\right] & {\left[A_{12}\right]} \\
{\left[A_{21}\right]} & {[0]}
\end{array}\right] \cdot\left[\begin{array}{l}
{[d Q]} \\
{[d H]}
\end{array}\right]=\left[\begin{array}{l}
{[d E]} \\
{[d q]}
\end{array}\right]
$$

Donde $d E$ representa el desbalance de energía por unidad de peso en cada tubo y $d q$ representa el desbalance de caudal en cada nudo. Ambos términos quedan definidos según las siguientes ecuaciones:

$$
\begin{gathered}
{[d E]=\left[A_{11}\right] \cdot\left[Q_{i}{ }^{T}\right]+\left[A_{12}\right] \cdot\left[H_{i}{ }^{T}\right]+\left[A_{10}\right] \cdot\left[H_{0}\right]} \\
{[d q]=\left[A_{21}\right] \cdot\left[Q_{i}{ }^{T}\right]-[q]}
\end{gathered}
$$

A partir de este punto, el método del gradiente se convierte en un proceso iterativo con:

$$
\begin{aligned}
& {[d Q]=\left[Q_{i}{ }^{T+1}\right]-\left[Q_{i}{ }^{T}\right]} \\
& {[d H]=\left[H_{i}{ }^{T+1}\right]-\left[H_{i}{ }^{T}\right]}
\end{aligned}
$$

Cuya resolución se obtendrá con la sustitución de las ecuaciones 4.10, 4.11, 4.12 y 4.13 en el sistema de ecuaciones mostrado en 4.9.

El desarrollo del método del gradiente para el problema que presenta un sistema de distribución está recogido en el estudio planteado por Todini \& Rossman (2013), y se presenta a continuación: 


$$
\left[\begin{array}{ccc}
A_{11} & \vdots & A_{12} \\
\cdots & \cdots & \cdots \\
A_{21} & \vdots & 0
\end{array}\right] \cdot\left[\begin{array}{c}
Q \\
\cdots \\
H
\end{array}\right]+\left[\begin{array}{c}
A_{10} \cdot H_{0} \\
q
\end{array}\right]=\left[\begin{array}{c}
f_{1} \\
\cdots \\
f_{2}
\end{array}\right]
$$

Donde $f_{1}$ y $f_{2}$ indican cuán cerca se encuentra el sistema de cero, según la solución aproximada elegida de $Q$ y $H$.

Aplicando Newton-Raphson al anterior sistema, se obtiene como resultado el siguiente conjunto de ecuaciones para las correcciones de caudales y alturas en la iteración $\tau$ del proceso.

$$
\left[\begin{array}{cc}
D_{11}^{\tau} & A_{12} \\
A_{21} & 0
\end{array}\right] \cdot\left[\begin{array}{l}
d Q \\
d H
\end{array}\right]=\left[\begin{array}{l}
f_{1}^{\tau} \\
f_{2}^{\tau}
\end{array}\right]
$$

Con:

$$
\begin{gathered}
d Q=Q^{\tau}-Q^{\tau+1} \\
d H=H^{\tau}-H^{\tau+1} \\
f_{1}^{\tau}=A_{11}^{\tau} Q^{\tau}+A_{12} H^{\tau}+A_{10} H_{0} \\
f_{2}^{\tau}=A_{12} Q^{\tau}+q
\end{gathered}
$$

donde $D_{11}$ es una matriz diagonal de dimensión $p$, que representa el jacobiano de $A_{11} Q$. Operando sobre la ecuación 4.15:

$$
\begin{gathered}
D_{11}{ }^{\tau} d Q+A_{12} d H=f_{1}{ }^{\tau} \\
A_{21} d Q=f_{2}{ }^{\tau}
\end{gathered}
$$

y despejando en la ecuación 4.20:

$$
d Q=\left(D_{11}^{\tau}\right)^{-1} \cdot\left(f_{1}^{\tau}-A_{12} d H\right)
$$

Sustituyendo 4.22 en 4.21 , y operando:

$$
\begin{gathered}
A_{21}\left\{\left(D_{11}^{\tau}\right)^{-1} \cdot\left(f_{1}{ }^{\tau}-A_{12} d H\right)\right\}=f_{2}{ }^{\tau} \\
A_{21}\left(D_{11}^{\tau}\right)^{-1} f_{1}{ }^{\tau}-A_{21}\left(D_{11}^{\tau}\right)^{-1} A_{12} d H=f_{2}{ }^{\tau}
\end{gathered}
$$




$$
\begin{gathered}
d H=\left(A_{21}\left(D_{11}^{\tau}\right)^{-1} A_{12}\right)^{-1}\left(-f_{2}{ }^{\tau}+A_{21}\left(D_{11}^{\tau}\right)^{-1} f_{1}{ }^{\tau}\right) \\
H^{\tau+1}=H^{\tau}-\left(A_{21}\left(D_{11}^{\tau}\right)^{-1} A_{12}\right)^{-1}\left(-f_{2}{ }^{\tau}+A_{21}\left(D_{11}^{\tau}\right)^{-1} f_{1}^{\tau}\right) \\
H^{\tau+1}=H^{\tau}-\left(A_{21}\left(D_{11}^{\tau}\right)^{-1} A_{12}\right)^{-1}\left(-A_{12} Q^{\tau}-q\right. \\
\left.+A_{21}\left(D_{11}^{\tau}\right)^{-1}\left(A_{11}^{\tau} Q^{\tau}+A_{12} H^{\tau}+A_{10} H_{0}\right)\right) \\
\text { Ahora, sustituyendo la ecuación } 4.24 \text { en } 4.20 \text { y operando: } \\
H^{\tau+1}=\left(A_{21}\left(D_{11}^{\tau}\right)^{-1} A_{12}\right)^{-1}\left(A_{12} Q^{\tau}+q-A_{21}\left(D_{11}^{\tau}\right)^{-1}\left(A_{11}^{\tau} Q^{\tau}+A_{10} H_{0}\right)\right) \\
D_{11}{ }^{\tau} d Q+A_{12}\left(A_{21}\left(D_{11}^{\tau}\right)^{-1} A_{12}\right)^{-1}\left(-f_{2}{ }^{\tau}+A_{21}\left(D_{11}^{\tau}\right)^{-1} f_{1}{ }^{\tau}\right)=f_{1}^{\tau} \\
d Q=\left(D_{11}^{\tau}\right)^{-1}\left\{f_{1}^{\tau}-A_{12}\left(A_{21}\left(D_{11}^{\tau}\right)^{-1} A_{12}\right)^{-1}\left(-f_{2}{ }^{\tau}+A_{21}\left(D_{11}^{\tau}\right)^{-1} f_{1}^{\tau}\right)\right\} \\
Q^{\tau}-Q^{\tau+1}=\left(D_{11}^{\tau}\right)^{-1}\left\{A_{11}^{\tau} Q^{\tau}+A_{10} H_{0}-A_{12}\left(A_{21}\left(D_{11}^{\tau}\right)^{-1} A_{12}\right)^{-1}\right. \\
\left.\cdot\left(-A_{12} Q^{\tau}-q+A_{21}\left(D_{11}^{\tau}\right)^{-1}\left(A_{11}^{\tau} Q^{\tau}+A_{10} H_{0}\right)\right)\right\} \\
Q^{\tau+1}=Q^{\tau}-\left(D_{11}^{\tau}\right)^{-1}\left\{A_{11}^{\tau} Q^{\tau}+A_{10} H_{0}-A_{12}\left(A_{21}\left(D_{11}^{\tau}\right)^{-1} A_{12}\right)^{-1}\right. \\
\left.\cdot\left(-A_{12} Q^{\tau}-q+A_{21}\left(D_{11}^{\tau}\right)^{-1}\left(A_{11}^{\tau} Q^{\tau}+A_{10} H_{0}\right)\right)\right\}
\end{gathered}
$$

Una vez obtenidas las ecuaciones 4.25 y 4.27 , la última fase del problema consiste en un proceso iterativo que parte de la solución aproximada $\left(Q^{\tau}, H^{\tau}\right)$ y desprende en cada paso la solución $\left(Q^{\tau+1}, H^{\tau+1}\right)$ más cercana a la solución del problema. El proceso seguirá iterando hasta que ambas soluciones converjan, en dicho caso la solución $\left(Q^{\tau+1}, H^{\tau+1}\right)$ será la solución al problema planteado.

\subsection{Estudio de la sensibilidad de la red}

Como se desprende de las ecuaciones 4.1 y 4.2 y del desarrollo de sus resoluciones, todos los elementos que forman las redes de distribución de agua están relacionados. De hecho, una variación en el funcionamiento de cualquiera de ellos afectará al comportamiento del resto. Analizar la sensibilidad de la red, es decir, cuantificar cuánto varía cada elemento sobre el conjunto de una red ( $F u$ et al., 2012), o cuán importante es un elemento para el funcionamiento global. Dicha variabilidad se emplea en el diseño de la sectorización de una red para elegir qué tuberías pueden estar cerradas o abiertas, evitando así los procesos heurísticos seguidos con dicho fin (Fu et al., 2012).

Para abordar el análisis de la sensibilidad de la red, este estudio analiza el efecto de un aumento de la demanda en cualquier lugar de la red sobre la variación del flujo y presión causada. Dicho análisis de sensibilidad se estructura en tres pasos. 
- Paso 1 - Situación actual: El primer paso consiste en resolver el funcionamiento normal de la red de distribución, obtener la altura piezométrica en los nudos (vector $H$, ecuación 4.14) y el caudal que circula a través de las líneas (vector $Q$, ecuación 4.14). Este es el escenario base para el cual se calcula la sensibilidad del sistema.

Planteando este método de análisis para un ejemplo sencillo (Figura 4.1, red compuesta por 4 nudos, 5 tuberías y un depósito de cabecera), el funcionamiento normal queda definido por la siguiente estructura:

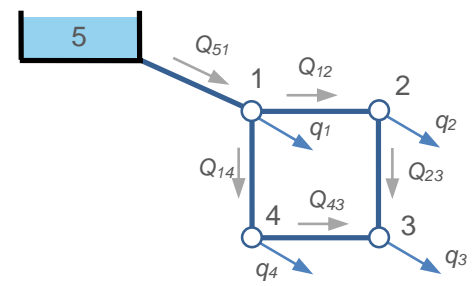

Figura 4.1. Red de distribución tipo

Para este caso, y en condiciones normales de funcionamiento, la ecuación de conservación de la masa para cada nudo será:

$$
\sum_{k=1}^{n_{i}} Q_{i k_{0}}+q_{i}=0
$$

Nótese que se ha marcado con subíndice $0\left(Q_{i k_{0}}\right)$ aquellas variables que hacen referencia al escenario de funcionamiento normal de la red.

- Paso 2 - Incremento del consumo: Si en el nudo $i$, la demanda actual $\left(q_{i}\right)$ se incrementa un cierto valor $\left(\varphi_{q_{i}}\right)$, esta variación deberá incluirse formalmente en una nueva demanda $\left(q_{i}+\varphi_{q_{i}}\right)$, afectará también a la altura piezométrica del mismo nudo $i\left(H_{i_{0}}+\right.$ $\left.\varphi_{H_{i_{i}}}\right)$, a la del resto de nudos $j$ de la red $\left(H_{j_{0}}+\varphi_{H_{j_{i}}}\right)$, y al caudal circulante de cualquier tubería $i k\left(Q_{i k_{0}}+\varphi_{Q_{i k_{i}}}\right)$.

Nótese que la nueva formulación de la ecuación de conservación de masa en el nudo iserá:

$$
\sum_{k=1}^{n_{i}}\left(Q_{i k_{0}}+\varphi_{Q_{i k_{i}}}\right)+q_{i}+\varphi_{q_{i}}=0
$$

Donde $\varphi_{Q_{i k_{i}}}$ corresponde con la variación del caudal en la línea que conecta los nudos i y $k$, producida por la alteración de la demanda del nudo $i$.

A su vez, la ecuación de conservación de masa en cualquier nudo $j$ (debido al incremento de demanda en el nudo i) será:

$$
\sum_{k=1}^{n_{j}}\left(Q_{j k_{0}}+\varphi_{Q_{j k_{i}}}\right)+q_{j}=0
$$

En paralelo, la ecuación de conservación de energía en cualquier línea ij tras el incremento de la demanda en el nudo $i$, será: 


$$
\left(H_{i_{0}}+\varphi_{H_{i_{i}}}\right)-\left(H_{j_{0}}+\varphi_{H_{j_{i}}}\right)=r_{i j}\left(Q_{i j_{0}}+\varphi_{Q_{i j_{i}}}\right)\left|Q_{i j_{0}}+\varphi_{Q_{i j_{i}}}\right|
$$

La variación del caudal en la línea ij puede ser positiva o negativa, es decir, la variación de la demanda en el nudo $i$ puede hacer que el flujo de la línea ij se vea incrementado o disminuido. Por ello, es necesario aproximar la expresión 4.31 según se indica:

$$
\begin{gathered}
\left(H_{i_{0}}+\varphi_{H_{i_{i}}}\right)-\left(H_{j_{0}}+\varphi_{H_{j_{i}}}\right)-\vartheta_{i j} r_{i j} \\
\cdot\left(Q_{i j_{0}}\left|Q_{i j_{0}}\right|+\frac{{Q_{i j_{0}}}^{2} \cdot \varphi_{Q_{i j_{i}}}}{\left|Q_{i j_{0}}\right|}+\frac{Q_{i j_{0}} \cdot \varphi_{Q_{i j_{i}}}{ }^{2}}{\left|Q_{i j_{0}}\right|}+\varphi_{Q_{i j_{i}}}\left|Q_{i j_{0}}\right|\right)=0
\end{gathered}
$$

Donde $\vartheta_{i j}$ es un término que adopta el valor de $\frac{Q_{i j_{0}} \cdot \varphi_{Q_{i j_{i}}}}{\left|Q_{i j_{0}}\right| \cdot\left|\varphi_{Q_{i j_{i}}}\right|}$ si $\left|\varphi_{Q_{i j_{i}}}\right|>\left|\mathrm{Q}_{\mathrm{ij}}\right|$ y la unidad en caso contrario.

Siguiendo con la red del ejemplo, véase como afectará un incremento en el consumo del nudo 2 a todas las variables del sistema:

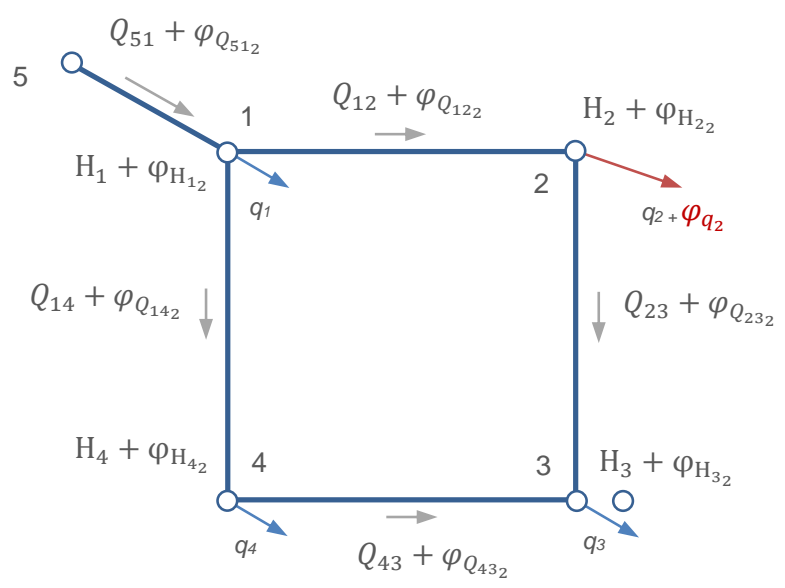

Figura 4.2. Red de distribución tipo ante un incremento del consumo en el nudo 2

- Paso 3 - Cálculo de la sensibilidad de la red: a partir de las ecuaciones presentadas en el paso anterior, el cálculo de la sensibilidad de todos los elementos de la red ante la variación de la demanda del nudo $i$, es relativamente sencillo. Para ello, será necesario resolver la ecuación 4.29, para los nudos, y la 4.31, para las líneas. Tras ello, se obtendrá la variación del flujo de cada tubería $\varphi_{Q_{i j_{i}}}$ y la variación de la altura piezométrica de cada nudo $\varphi_{H_{j_{i}}}$, para el aumento de demanda fijado. Pero esta sensibilidad calculada solo analiza cómo se ven afectados todos los elementos ante la variación de demanda en un único punto, en el nudo $i$.

Como la red consta de $n$ nudos, el proceso se puede repetir $n$ veces suponiendo la misma variación de la demanda en cada uno de los nudos de consumo. De este modo, se obtiene una visión total de la sensibilidad de la red, y para cada elemento se obtiene un rango de variación total. Ahora bien, pese a que este análisis es factible (a partir de las ecuaciones presentadas), puede que no sea muy operativo ya que el tiempo de cálculo puede ser excesivo. 
Por esta razón, el método propuesto incluye una simplificación para resolver las $n$ repeticiones tan solo una vez. En lugar de trabajar por separado con la variación específica en cada elemento causada por el aumento de la demanda en cada nudo, se considera el promedio de todas estas variaciones. Así, es necesario introducir la sensibilidad media de la altura piezométrica en cada nudo $i$ :

$$
\varphi_{H_{i}}=\frac{\sum_{m=1}^{n} \varphi_{H_{i_{m}}}}{n}
$$

Y la sensibilidad media del caudal en cada tubería ik, según la ecuación 4.34:

$$
\varphi_{Q_{i k}}=\frac{\sum_{m=1}^{n} \varphi_{Q_{i k_{m}}}}{n}
$$

Para ello, y tras estas consideraciones, es necesario mostrar las ecuaciones de conservación de masa para el nudo $i$, bajo cada uno de los escenarios posibles, es decir, bajo cada caso de aumento de la demanda $\left(\varphi_{q_{i}}\right)$ en cada uno de los nudos de

\begin{tabular}{|c|c|c|}
\hline Nudo & $\begin{array}{l}\text { Aumento de } \\
\text { demanda } \\
\text { en el nudo: }\end{array}$ & Ecuación de conservación de masa (para el nudo I) \\
\hline \multirow{8}{*}{$i$} & 1 & $\left(Q_{i 1_{0}}+\varphi_{Q_{i 1_{1}}}\right)+\cdots+\left(Q_{i k_{0}}+\varphi_{Q_{i k_{1}}}\right)+\cdots+\left(Q_{i n_{i_{0}}}+\varphi_{Q_{i n_{i_{1}}}}\right)+\left(q_{i}\right)=0$ \\
\hline & 2 & $\left(Q_{i 1_{0}}+\varphi_{Q_{i 1_{2}}}\right)+\cdots+\left(Q_{i k_{0}}+\varphi_{Q_{i k_{2}}}\right)+\cdots+\left(Q_{i n_{i_{0}}}+\varphi_{Q_{i n_{i_{2}}}}\right)+\left(q_{i}\right)=0$ \\
\hline & ... & $\ldots$ \\
\hline & $m$ & $\left(Q_{i 1_{0}}+\varphi_{Q_{i 1_{m}}}\right)+\cdots+\left(Q_{i k_{0}}+\varphi_{Q_{i k_{m}}}\right)+\cdots+\left(Q_{i n_{i_{0}}}+\varphi_{Q_{i n_{i m}}}\right)+\left(q_{i}\right)=0$ \\
\hline & ... & $\ldots$ \\
\hline & $i$ & $\left(Q_{i 1_{0}}+\varphi_{Q_{i 1_{i}}}\right)+\cdots+\left(Q_{i k_{0}}+\varphi_{Q_{i k_{i}}}\right)+\cdots+\left(Q_{i n_{i_{0}}}+\varphi_{Q_{i n_{i}}}\right)+\left(q_{i}+\varphi_{q_{i}}\right)=0$ \\
\hline & & $\cdots$ \\
\hline & $n$ & $\left(Q_{i 1_{0}}+\varphi_{Q_{i 1_{n}}}\right)+\cdots+\left(Q_{i k_{0}}+\varphi_{Q_{i k_{n}}}\right)+\cdots+\left(Q_{i n_{i_{0}}}+\varphi_{Q_{i i_{i_{n}}}}\right)+\left(q_{i}\right)=0$ \\
\hline
\end{tabular}
la red. Así, el conjunto completo de $n$ ecuaciones para el nudo i será:

Sumando las $n$ ecuaciones anteriores para el nudo $i$, el resultado es:

$$
\begin{gathered}
\sum_{k=1}^{n_{i}}\left(n Q_{i k_{0}}\right)+\sum_{k=1}^{n_{i}}\left(\sum_{m=1}^{n} \varphi_{Q_{i k_{m}}}\right)+n q_{i}+\varphi_{q_{i}}=0 \\
\sum_{k=1}^{n_{i}}\left(n Q_{i k_{0}}+\sum_{m=1}^{n} \varphi_{Q_{i k_{m}}}\right)+n q_{i}+\varphi_{q_{i}}=0
\end{gathered}
$$

Si ahora se divide por $n$ la ecuación 4.36 y se incluye el término de sensibilidad media para cada tubería (ecuación 4.34), se obtiene la ecuación de conservación de masas 
me para el nudo $i$ ante un incremento en la demanda de valor $\varphi_{q_{i}}$ en cualquier punto de la red:

$$
\sum_{k=1}^{n_{i}}\left(Q_{i k_{0}}+\varphi_{Q_{i k}}\right)+q_{i}+\frac{\varphi_{q_{i}}}{n}=0
$$

Se procede análogamente para todos los nudos de la red. Con lo que, si se adopta un incremento de la demanda de valor $\varphi_{q}$, (constante y conocido), el conjunto completo de las ecuaciones de conservación de masa medias para todos los nudos de la red se muestra en 4.38:

\begin{tabular}{|c|c|}
\hline Nudo id & Ecuación de conservación de masa \\
\hline 1 & $\sum_{k=1}^{n_{1}}\left(Q_{1 k_{0}}+\varphi_{Q_{1 k}}\right)+q_{1}+\frac{\varphi_{q}}{n}=0$ \\
\hline 2 & $\sum_{k=1}^{n_{2}}\left(Q_{2 k_{0}}+\varphi_{Q_{2 k}}\right)+q_{2}+\frac{\varphi_{q}}{n}=0$ \\
\hline$\ldots$ & $\ldots$ \\
\hline$m$ & $\sum_{k=1}^{n_{m}}\left(Q_{m k_{0}}+\varphi_{Q_{m k}}\right)+q_{m}+\frac{\varphi_{q}}{n}=0$ \\
\hline$n$ & $\ldots$ \\
\hline
\end{tabular}

El sistema de ecuaciones 4.38 está compuesto por $n$ ecuaciones con $p$ incógnitas, la sensibilidad media del caudal en las tuberías $\left(\varphi_{Q_{i k}}\right)$. De acuerdo con la formulación matricial usada por Todini \& Rossman (2013) este sistema puede expresarse como:

$$
\left[A_{21}\right]\left[Q+\varphi_{Q}\right]+\left[q+\frac{\varphi_{q}}{n}\right]=0
$$

donde $A_{21}$ es una matriz, de dimensiones [ $\left.n, p\right]$, que relaciona $n$ nudos con $p$ tuberías y como valores de la celda $A_{21}(q, r)$ toma - 1 si el flujo de la tubería $r$ abandona el nudo $q$, 0 si la tubería $r$ no conecta con el nudo $q$ y 1 si el flujo de la tubería $r$ entra al nudo $q, Q$ es el vector de los caudales de las líneas, $\varphi_{Q}$ es el vector de la sensibilidad media de caudales en las líneas $\left(\varphi_{Q_{i j}}\right)$, $q$ es el vector con las demandas nodales y $\varphi_{q}$ es el incremento de demanda.

Finalmente, la ecuación 4.39 se transforma en la siguiente expresión:

$$
\left[A_{21} \varphi_{Q}\right]=-\left[A_{21} Q\right]-\left[q_{i}+\frac{\varphi_{q}}{n}\right]
$$


Si ahora se considera la ecuación de conservación de energía para cada tubería ij, en el caso de incremento de la demanda ocurrida en cada uno de los nudos de la red, el conjunto completo de $n$ ecuaciones para la tubería ij será:

\begin{tabular}{|c|c|c|}
\hline Tubería & $\begin{array}{l}\text { Aumento de } \\
\text { demanda en } \\
\text { el nudo: }\end{array}$ & Ecuación de conservación de energía (para la tubería ij) \\
\hline \multirow{8}{*}{$i j$} & 1 & $\left(H_{i_{0}}+\varphi_{H_{i_{1}}}\right)-\left(H_{j_{0}}+\varphi_{H_{j_{1}}}\right)=r_{i j}\left(Q_{i j_{0}}+\varphi_{Q_{i j_{1}}}\right)\left|Q_{i j_{0}}+\varphi_{Q_{i j_{1}}}\right|$ \\
\hline & 2 & $\left(H_{i_{0}}+\varphi_{H_{i_{2}}}\right)-\left(H_{j_{0}}+\varphi_{H_{j_{2}}}\right)=r_{i j}\left(Q_{i j_{0}}+\varphi_{Q_{i j_{2}}}\right)\left|Q_{i j_{0}}+\varphi_{Q_{i j_{2}}}\right|$ \\
\hline & & $\ldots$ \\
\hline & $i$ & $\left(H_{i_{0}}+\varphi_{H_{i_{i}}}\right)-\left(H_{j_{0}}+\varphi_{H_{j_{i}}}\right)=r_{i j}\left(Q_{i j_{0}}+\varphi_{Q_{i j_{i}}}\right)\left|Q_{i j_{0}}+\varphi_{Q_{i j_{i}}}\right|$ \\
\hline & $\ldots$ & $\ldots$ \\
\hline & $j$ & $\left(H_{i_{0}}+\varphi_{H_{i_{j}}}\right)-\left(H_{j_{0}}+\varphi_{H_{j_{j}}}\right)=r_{i j}\left(Q_{i j_{0}}+\varphi_{Q_{i j_{j}}}\right)\left|Q_{i j_{0}}+\varphi_{Q_{i j_{j}}}\right|$ \\
\hline & $\ldots$ & $\ldots$ \\
\hline & $n$ & $\left(H_{i_{0}}+\varphi_{H_{i_{n}}}\right)-\left(H_{j_{0}}+\varphi_{H_{j_{n}}}\right)=r_{i j}\left(Q_{i j_{0}}+\varphi_{Q_{i j_{n}}}\right)\left|Q_{i j_{0}}+\varphi_{Q_{i j_{n}}}\right|$ \\
\hline
\end{tabular}

Las ecuaciones del sistema 4.41 no se pueden sumar fácilmente, por lo que para ello se considera la siguiente operación:

$$
(Q+\varphi)|Q+\varphi|=\vartheta\left(Q|Q|+\frac{Q^{2} \varphi}{|Q|}+\frac{Q \varphi^{2}}{|Q|}+\varphi|Q|\right)
$$

donde:

- $\vartheta=\frac{Q \varphi}{|Q||\varphi|}$, si $|\varphi|>|Q|$

- $\vartheta=1, \operatorname{si}|\varphi| \leq|Q|$

Así, el nuevo sistema de $n$ ecuaciones de conservación de energía para la línea ij es:

\begin{tabular}{|c|c|c|}
\hline Tubería & $\begin{array}{l}\text { Aumento } \\
\text { demanda } \\
\text { en el } \\
\text { nudo: }\end{array}$ & Ecuación de conservación de energía (para la tubería ij) \\
\hline \multirow{5}{*}{$i j$} & 1 & $\left(H_{i_{0}}+\varphi_{H_{i_{1}}}\right)-\left(H_{j_{0}}+\varphi_{H_{j_{1}}}\right)-\vartheta_{\mathrm{ij}} \mathrm{r}_{\mathrm{ij}} \cdot\left(\mathrm{Q}_{i j_{0}}\left|\mathrm{Q}_{i j_{0}}\right|+\frac{\mathrm{Q}_{i j_{0}}{ }^{2} \cdot \varphi_{Q_{i j_{1}}}}{\left|\mathrm{Q}_{i j_{0}}\right|}+\frac{\mathrm{Q}_{i j_{0}} \cdot \varphi_{Q_{i j_{1}}}{ }^{2}}{\left|\mathrm{Q}_{i j_{0}}\right|}+\varphi_{Q_{i j_{1}}}\left|Q_{i j_{0}}\right|\right)=0$ \\
\hline & 2 & $\left(H_{i_{0}}+\varphi_{H_{i_{2}}}\right)-\left(H_{j_{0}}+\varphi_{H_{j_{2}}}\right)-\vartheta_{\mathrm{ij}} \mathrm{r}_{\mathrm{ij}} \cdot\left(\mathrm{Q}_{i j_{0}}\left|\mathrm{Q}_{i j_{0}}\right|+\frac{\mathrm{Q}_{i j_{0}}{ }^{2} \cdot \varphi_{Q_{i j_{2}}}}{\left|\mathrm{Q}_{i j_{0}}\right|}+\frac{\mathrm{Q}_{i j_{0}} \cdot \varphi_{Q_{i j_{2}}}{ }^{2}}{\left|\mathrm{Q}_{i j_{0}}\right|}+\varphi_{Q_{i j_{2}}}\left|Q_{i j_{0}}\right|\right)=0$ \\
\hline & $\cdots$ & $\cdots$ \\
\hline & $i$ & $\left(H_{i_{0}}+\varphi_{H_{i_{i}}}\right)-\left(H_{j_{0}}+\varphi_{H_{j_{i}}}\right)-\vartheta_{\mathrm{ij}} \mathrm{r}_{\mathrm{ij}} \cdot\left(\mathrm{Q}_{i j_{0}}\left|\mathrm{Q}_{i j_{0}}\right|+\frac{\mathrm{Q}_{i j_{0}}{ }^{2} \cdot \varphi_{Q_{i j_{i}}}}{\left|\mathrm{Q}_{i j_{0}}\right|}+\frac{\mathrm{Q}_{i j_{0}} \cdot \varphi_{Q_{i j_{i}}}{ }^{2}}{\left|\mathrm{Q}_{i j_{0}}\right|}+\varphi_{Q_{i j_{i}}}\left|\mathrm{Q}_{i j_{0}}\right|\right)=0$ \\
\hline & $\cdots$ & $\ldots$ \\
\hline
\end{tabular}




\begin{tabular}{|c|c|c|}
\hline$j$ & $\left(H_{i_{0}}+\varphi_{H_{i_{j}}}\right)-\left(H_{j_{0}}+\varphi_{H_{j_{j}}}\right)-\vartheta_{\mathrm{ij}} \mathrm{r}_{\mathrm{ij}}$ & $\left(Q_{i j_{0}}\left|Q_{i j_{0}}\right|+\frac{Q_{i j_{0}}{ }^{2} \cdot \varphi_{Q_{i j_{j}}}}{\left|Q_{i j_{0}}\right|}+\frac{Q_{i j_{0}} \cdot \varphi_{Q_{i j_{j}}}{ }^{2}}{\left|Q_{i j_{0}}\right|}+\varphi_{Q_{i j_{j}}}\left|Q_{i j_{0}}\right|\right)=0$ \\
\hline$\ldots$ & & $\ldots$ \\
\hline$n$ & $\left(H_{i_{0}}+\varphi_{H_{i_{n}}}\right)-\left(H_{j_{0}}+\varphi_{H_{j_{n}}}\right)-\vartheta_{\mathrm{ij}} \mathrm{r}_{\mathrm{ij}}$ & $\left.Q_{i j_{0}}\left|Q_{i j_{0}}\right|+\frac{Q_{i j_{0}}{ }^{2} \cdot \varphi_{Q_{i j_{n}}}}{\left|Q_{i j_{0}}\right|}+\frac{Q_{i j_{0}} \cdot \varphi_{Q_{i j_{n}}}{ }^{2}}{\left|Q_{i j_{0}}\right|}+\varphi_{Q_{i j_{n}}}\left|Q_{i j_{0}}\right|\right)=0$ \\
\hline
\end{tabular}

Antes de sumar las $n$ ecuaciones del sistema 4.43 se debe tener presente que $\frac{\sum_{m=1}^{n} \varphi_{Q_{i j_{m}}}{ }^{2}}{n}$ es la media de los cuadrados de $\varphi_{Q_{i j_{m}}}$. Sin embargo, siendo $\varphi_{Q_{i j}}$ la variable final a resolver, es necesario asumir la simplificación $\frac{\sum_{m=1}^{n} \varphi_{Q_{i j m}}{ }^{2}}{n} \approx \varphi_{Q_{i j}}{ }^{2}$. Esto se basa en el hecho de que, en comparación con los otros términos dentro del paréntesis, $\frac{\mathrm{Q}_{i j_{0}} \cdot \varphi_{Q_{i j_{n}}}{ }^{2}}{\left|\mathrm{Q}_{i j_{0}}\right|}$ es el menos relevante debido a la magnitud de $\varphi_{Q_{i j}}{ }^{2}$ (comparado con $\varphi_{Q_{i j}}$ ), $0 \frac{Q_{i j_{0}}}{\left|\mathrm{Q}_{i j_{0}}\right|}$ (comparado con $\left|\mathrm{Q}_{i j_{0}}\right|$ ). El resultado final de la suma de las $n$ ecuaciones para la tubería ij es:

$$
\begin{aligned}
& n H_{i_{0}}+\sum_{m=1}^{n} \varphi_{H_{i_{m}}}-n H_{j_{0}}-\sum_{m=1}^{n} \varphi_{H_{j_{m}}}-
\end{aligned}
$$

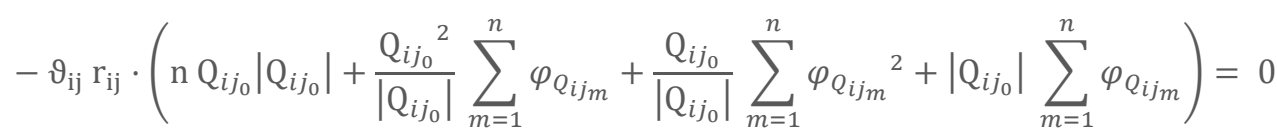

Introduciendo las sensibilidades medias (ecuaciones 4.334 .34 y 4.34) en la ecuación 4.44 y dividendo la ecuación resultante entre $n$, se obtiene la ecuación de conservación de energía media para cada tubería ij:

$$
\begin{gathered}
\left(H_{i_{0}}+\varphi_{H_{i}}\right)-\left(H_{j_{0}}+\varphi_{H_{j}}\right)-\vartheta_{\mathrm{ij}} \mathrm{r}_{\mathrm{ij}} \cdot\left(Q_{i j_{0}}\left|\mathrm{Q}_{i j_{0}}\right|+\frac{\mathrm{Q}_{i j_{0}}{ }^{2}}{\left|\mathrm{Q}_{i j_{0}}\right|} \varphi_{Q_{i j}}+\frac{\mathrm{Q}_{i j_{0}}}{\left|\mathrm{Q}_{i j_{0}}\right|} \varphi_{Q_{i j}}{ }^{2}+\left|Q_{i j_{0}}\right| \varphi_{Q_{i j}}\right) \\
=0 \\
H_{i_{0}}+\varphi_{H_{i}}-H_{j_{0}}-\varphi_{H_{j}}-\vartheta_{\mathrm{ij}} \mathrm{r}_{\mathrm{ij}} \cdot\left(\mathrm{Q}_{i j_{0}}\left|Q_{i j_{0}}\right|+\frac{Q_{i j_{0}}{ }^{2}}{\left|Q_{i j_{0}}\right|} \varphi_{Q_{i j}}+\frac{\mathrm{Q}_{i j_{0}}}{\left|\mathrm{Q}_{i j_{0}}\right|} \varphi_{Q_{i j}}{ }^{2}+\left|Q_{i j_{0}}\right| \varphi_{Q_{i j}}\right)=0 \\
\vartheta_{\mathrm{ij}} \mathrm{r}_{\mathrm{ij}} \cdot\left(2\left|\mathrm{Q}_{i j_{0}}\right|+\frac{Q_{i j_{0}}}{\left|Q_{i j_{0}}\right|} \varphi_{Q_{i j}}\right) \varphi_{Q_{i j}}-\varphi_{H_{i}}+\varphi_{H_{j}}+\vartheta_{\mathrm{ij}} \mathrm{r}_{\mathrm{ij}} \mathrm{Q}_{i j_{0}}\left|\mathrm{Q}_{i j_{0}}\right|-H_{i_{0}}+H_{j_{0}}=0
\end{gathered}
$$

Si ahora se extiende este planteamiento al resto de tuberías de la red, se obtiene el conjunto completo de ecuaciones de conservación de energía media para toda la red:

\begin{tabular}{|c|c|c|}
\hline \multicolumn{2}{|c|}{ Tubería } & \multirow{2}{*}{ Ecuación de conservación de energía (para la tubería $r \equiv i j$ ) } \\
\cline { 1 - 2 } Id & Nudos & \\
\hline 1 & $\ldots$ & \\
\hline 2 & $\ldots$ & $\ldots$ \\
\hline
\end{tabular}




\begin{tabular}{|c|c|c|}
\hline$\ldots$ & $\cdots$ & $\cdots$ \\
\hline$r$ & $i j$ & $\vartheta_{\mathrm{ij}} \mathrm{r}_{\mathrm{ij}} \cdot\left(2\left|\mathrm{Q}_{i j_{0}}\right|+\frac{\mathrm{Q}_{i j_{0}}}{\left|\mathrm{Q}_{i j_{0}}\right|} \varphi_{Q_{i j}}\right) \varphi_{Q_{i j}}-\varphi_{H_{i}}+\varphi_{H_{j}}+\vartheta_{\mathrm{ij}} \mathrm{r}_{\mathrm{ij}} \mathrm{Q}_{i j_{0}}\left|\mathrm{Q}_{i j_{0}}\right|-H_{i_{0}}+H_{j_{0}}$ \\
\hline$\ldots$ & $\cdots$ & $\cdots$ \\
\hline$p$ & $\ldots$ & $\cdots$ \\
\hline
\end{tabular}

El sistema 4.46 está compuesto por $p$ ecuaciones, con $(n+p)$ incógnitas: la sensibilidad promedio de la altura piezométrica para cada uno de los $n$ nudos $\left(\varphi_{H_{i}}\right)$, y la sensibilidad promedio del caudal para cada una de las $p$ tuberías $\left(\varphi_{Q_{i j}}\right)$. De acuerdo con la formulación matricial utilizada por Todini \& Rossman (2013), este sistema se puede expresar como:

$$
\left[\begin{array}{lll}
A_{11 \varphi} & \vdots & A_{12}
\end{array}\right]\left[\begin{array}{c}
\varphi_{Q} \\
\cdots \\
\varphi_{H}
\end{array}\right]+\left[\begin{array}{lll}
A_{11 \vartheta} & \vdots & A_{12}
\end{array}\right]\left[\begin{array}{c}
Q \\
\cdots \\
H
\end{array}\right]=-\left[A_{10} H_{0}\right]-\left[A_{10} \varphi_{H_{0}}\right]
$$

donde $A_{11 \varphi}$ es una matriz diagonal de dimensión $p$, cuyos elementos se refieren a una tubería $r$ que conecta los nudos ij y cuyo valor es:

$$
A_{11 \varphi}(r, r)=\vartheta_{\mathrm{ij}} \mathrm{r}_{\mathrm{ij}}\left(2\left|\mathrm{Q}_{i j_{0}}\right|+\frac{\mathrm{Q}_{i j_{0}}}{\left|\mathrm{Q}_{i j_{0}}\right|} \varphi_{Q_{i j}}\right),
$$

donde $A_{11 \vartheta}$ es una matriz diagonal de dimensión $p$, cuyos elementos se refieren a la tubería $r$ que conecta a los nudos ij y cuyo valor es:

$$
A_{11 \vartheta}(r, r)=\vartheta_{\mathrm{ij}} \mathrm{r}_{\mathrm{ij}}\left|\mathrm{Q}_{i j_{0}}\right|,
$$

donde $H$ es el vector de las alturas piezométricas de los nudos $H_{i_{0}}, \varphi_{H}$ es el vector de la sensibilidad de las alturas piezométricas de los nudos $\varphi_{H_{i}}, A_{10}$ es la matriz que relaciona tuberías con nudos de altura fija, $H_{0}$ es el vector de los nudos de altura fija, $\varphi_{H_{0}}$ es el vector de sensibilidades de las alturas piezométricas, con valores ceros en los nudos equipados con sensor de presión, $\varphi_{Q}, Q, q, \frac{\varphi_{q}}{n}$ y $A_{21}$ son los vectores y matrices explicados con anterioridad y $A_{12}=A_{21}{ }^{T}$.

La ecuación 4.47 puede ser organizada según se muestra:

$$
\left[\begin{array}{lll}
A_{11 \varphi} & \vdots & A_{12}
\end{array}\right]\left[\begin{array}{c}
\varphi_{Q} \\
\cdots \\
\varphi_{H}
\end{array}\right]=-\left[\begin{array}{lll}
A_{11 \vartheta} & \vdots & A_{12}
\end{array}\right]\left[\begin{array}{c}
Q \\
\cdots \\
H
\end{array}\right]-\left[A_{10} H_{0}\right]
$$

El sistema completo de ecuaciones de conservación se puede obtener mediante la fusión del sistema 4.40 y el sistema 4.50. El nuevo sistema consta de $n+p$ ecuaciones, con $n+p$ incógnitas, y puede tomar una formulación matricial como:

$$
\left[\begin{array}{ccc}
A_{11 \varphi} & \vdots & A_{12} \\
\cdots & \cdots & \cdots \\
A_{21} & \vdots & 0
\end{array}\right]\left[\begin{array}{c}
\varphi_{Q} \\
\cdots \\
\varphi_{H}
\end{array}\right]=-\left[\begin{array}{ccc}
A_{11 \vartheta} & \vdots & A_{12} \\
\cdots & \cdots & \cdots \\
A_{21} & \vdots & 0
\end{array}\right]\left[\begin{array}{c}
Q \\
\cdots \\
H
\end{array}\right]-\left[\begin{array}{c}
A_{10} H_{0} \\
\cdots \\
q+\frac{\varphi_{q}}{n}
\end{array}\right]
$$


Siendo $\varphi_{Q}$ y $\varphi_{H}$ los vectores de los valores desconocidos, el sistema 4.51 puede resolverse mediante el método de Newton-Raphson para obtener, finalmente, las sensibilidades medias ante una variación conocida, y de valor $\varphi_{q}$, para cada nudo y tubería de la red.

El método de Newton-Raphson, aplicado por Todini (2014) para la resolución de la modelación de los sistemas de distribución de agua potable, consiste en seguir de un proceso iterativo que, partiendo de una solución real dada, se obtenga como resultado de cada iteración, una solución más cercana a las raíces del problema.

Así, al dotar de una solución distinta a la raíz a la ecuación 4.51, se obtendrán unos residuos, vectores $f_{1}$ y $f_{2}$ :

$$
\left[\begin{array}{cc}
A_{11 \varphi} & A_{12} \\
A_{21} & 0
\end{array}\right] \cdot\left[\begin{array}{c}
\varphi_{Q} \\
\varphi_{H}
\end{array}\right]+\left[\begin{array}{c}
A_{10} \cdot H_{0}+A_{11 \vartheta} Q+A_{12} H \\
q+\frac{\varphi_{q}}{n}+A_{21} Q
\end{array}\right]=\left[\begin{array}{c}
f_{1} \\
f_{2}
\end{array}\right]
$$

donde $f_{1}=f_{1}\left(\varphi_{Q}, \varphi_{H}\right)$ y $f_{2}=f_{2}\left(\varphi_{Q}\right)$ indican cuán cerca están las raíces del sistema de ecuaciones, para cualquier valor inicial de $\varphi_{Q}$ y $\varphi_{H}$; y su valor es el siguiente:

$$
\begin{gathered}
f_{1}=A_{11 \varphi} \cdot \varphi_{Q}+A_{12} \cdot \varphi_{H}+\left\{A_{10} \cdot H_{0}+A_{11 \vartheta} \cdot Q+A_{12} \cdot H\right\} \\
f_{2}=A_{21} \cdot \varphi_{Q}+\left\{q+\frac{\varphi_{q}}{n}+A_{21} Q\right\}
\end{gathered}
$$

Siguiendo Newton-Raphson, se obtiene la siguiente expresión:

$$
\left[\begin{array}{cc}
D_{11 \varphi} & A_{12} \\
A_{21} & 0
\end{array}\right] \cdot\left[\begin{array}{l}
d \varphi_{Q} \\
d \varphi_{H}
\end{array}\right]=\left[\begin{array}{l}
-f_{1} \\
-f_{2}
\end{array}\right]
$$

con:

$$
\begin{aligned}
& d \varphi_{Q}=\varphi_{Q}^{T+1}-\varphi_{Q}^{T} \\
& d \varphi_{H}=\varphi_{H}^{T+1}-\varphi_{H}^{T}
\end{aligned}
$$

Desarrollando la ecuación 4.55:

$$
\begin{gathered}
D_{11 \varphi} \cdot d \varphi_{Q}+A_{12} \cdot d \varphi_{H}=-f_{1} \\
A_{21} \cdot d \varphi_{Q}=-f_{2}
\end{gathered}
$$

Despejando en la ecuación 4.57,

$$
d \varphi_{Q}=D_{11 \varphi}{ }^{-1} \cdot\left\{-f_{1}-A_{12} \cdot d \varphi_{H}\right\}
$$

Y sustituyendo 4.59 en 4.58:

$$
\begin{gathered}
A_{21} \cdot D_{11 \varphi}{ }^{-1} \cdot\left\{-f_{1}-A_{12} \cdot d \varphi_{H}\right\}=-f_{2} \\
A_{21} \cdot D_{11 \varphi}{ }^{-1} \cdot f_{1}+A_{21} \cdot D_{11 \varphi}{ }^{-1} \cdot A_{12} \cdot d \varphi_{H}=f_{2} \\
A_{21} \cdot D_{11 \varphi}{ }^{-1} \cdot A_{12} \cdot d \varphi_{H}=f_{2}-A_{21} \cdot D_{11 \varphi}{ }^{-1} \cdot f_{1} \\
d \varphi_{H}=\left(A_{21} \cdot D_{11 \varphi}{ }^{-1} \cdot A_{12}\right)^{-1} \cdot\left\{f_{2}-A_{21} \cdot D_{11 \varphi}{ }^{-1} \cdot f_{1}\right\}
\end{gathered}
$$


Sustituyendo $f_{1}$ y $f_{2}$ :

$$
\begin{gathered}
d \varphi_{H}=\left(A_{21} \cdot D_{11 \varphi}{ }^{-1} \cdot A_{12}\right)^{-1} \cdot\left\{\left(A_{21} \cdot \varphi_{Q}^{T}+\left\{q+\frac{\varphi_{q}}{n}+A_{21} \cdot Q\right\}\right)\right. \\
\left.-A_{21} \cdot D_{11 \varphi}{ }^{-1} \cdot\left(A_{11 \varphi} \cdot \varphi_{Q}^{T}+A_{12} \cdot \varphi_{H}^{T}+\left\{A_{10} \cdot H_{0}+A_{11 \vartheta} \cdot Q+A_{12} \cdot H\right\}\right)\right\} \\
\varphi_{H}^{T+1}-\varphi_{H}^{T}=\left(A_{21} \cdot D_{11 \varphi}{ }^{-1} \cdot A_{12}\right)^{-1} \cdot\left\{A_{21} \cdot \varphi_{Q}^{T}+\left(q+\frac{\varphi_{q}}{n}+A_{21} \cdot Q\right)\right. \\
-A_{21} \cdot D_{11 \varphi}{ }^{-1} \cdot A_{11 \varphi} \cdot \varphi_{Q}^{T}-A_{21} \cdot D_{11 \varphi}{ }^{-1} \cdot A_{12} \cdot \varphi_{H}^{T}-A_{21} \cdot D_{11 \varphi}{ }^{-1} \\
\left.\cdot\left(A_{10} \cdot H_{0}+A_{11 \vartheta} \cdot Q+A_{12} \cdot H\right)\right\} \\
\varphi_{H}^{T+1}=\left(A_{21} \cdot D_{11 \varphi}{ }^{-1} \cdot A_{12}\right)^{-1} \cdot\left\{A_{21} \cdot \varphi_{Q}^{T}+\left(q+\frac{\varphi_{q}}{n}+A_{21} \cdot Q\right)\right. \\
\left.-A_{21} \cdot D_{11 \varphi}{ }^{-1} \cdot A_{11 \varphi} \cdot \varphi_{Q}^{T}-A_{21} \cdot D_{11 \varphi}{ }^{-1} \cdot\left(A_{10} \cdot H_{0}+A_{11 \vartheta} \cdot Q+A_{12} \cdot H\right)\right\} \\
\varphi_{H}^{T+1}=\left(A_{21} \cdot D_{11 \varphi}{ }^{-1} \cdot A_{12}\right)^{-1} \cdot\left\{A _ { 2 1 } \cdot \left(\varphi_{Q}^{T}-D_{11 \varphi}{ }^{-1} \cdot A_{11 \varphi} \cdot \varphi_{Q}^{T}-D_{11 \varphi}{ }^{-1}\right.\right. \\
\left.\left.\cdot\left(A_{10} \cdot H_{0}+A_{11 \vartheta} \cdot Q+A_{12} \cdot H\right)\right)+\left(q+\frac{\varphi_{q}}{n}+A_{21} \cdot Q\right)\right\}
\end{gathered}
$$

Si introducimos la ecuación 4.60 en la 4.57:

$$
\begin{gathered}
D_{11 \varphi} \cdot d \varphi_{Q}+A_{12} \cdot\left(A_{21} \cdot D_{11 \varphi}{ }^{-1} \cdot A_{12}\right)^{-1} \cdot\left\{f_{2}-A_{21} \cdot D_{11 \varphi}{ }^{-1} \cdot f_{1}\right\}=-f_{1} \\
D_{11 \varphi} \cdot d \varphi_{Q}+A_{12} \cdot\left(A_{21} \cdot D_{11 \varphi}{ }^{-1} \cdot A_{12}\right)^{-1} \cdot\left\{A_{21} \cdot \varphi_{Q}^{T}+\left(q+\frac{\Delta \mathrm{q}}{n}+A_{21} Q_{0 i}\right)-A_{21} \cdot\right. \\
\left.D_{11 \varphi}{ }^{-1} \cdot\left(A_{11 \varphi} \cdot \varphi_{Q}^{T}+A_{12} \cdot \varphi_{H}^{T}+\left(A_{10} \cdot H_{0}+A_{11 \vartheta} \cdot Q+A_{12} \cdot H\right)\right)\right\}=-\left(A_{11 \varphi} \cdot \varphi_{Q}^{T}+\right. \\
\left.A_{12} \cdot \varphi_{H}^{T}+\left(A_{10} \cdot H_{0}+A_{11 \vartheta} \cdot Q+A_{12} \cdot H\right)\right) \\
d \varphi_{Q}=D_{11 \varphi}{ }^{-1} \cdot\left\{-A_{12} \cdot\left(A_{21} \cdot D_{11 \varphi}{ }^{-1} \cdot A_{12}\right)^{-1} \cdot\left\{A_{21} \cdot \varphi_{Q}^{T}+\left(q+\frac{\Delta \mathrm{q}}{n}+A_{21} \cdot Q_{0 i}\right)\right.\right. \\
\left.-A_{21} \cdot D_{11 \varphi}{ }^{-1} \cdot\left(A_{11 \varphi} \cdot \varphi_{Q}^{T}+A_{12} \cdot \varphi_{H}^{T}+\left(A_{10} \cdot H_{0}+A_{11 \vartheta} \cdot Q+A_{12} \cdot H\right)\right)\right\} \\
\left.-\left(A_{11 \varphi} \cdot \varphi_{Q}^{T}+A_{12} \cdot \varphi_{H}^{T}+\left(A_{10} \cdot H_{0}+A_{11 \vartheta} \cdot Q+A_{12} \cdot H\right)\right)\right\} \\
\varphi_{Q}^{T+1}-\varphi_{Q}^{T}=D_{11 \varphi}{ }^{-1} \cdot\left\{-A_{12} \cdot\left(A_{21} \cdot D_{11 \varphi}{ }^{-1} \cdot A_{12}\right)^{-1} \cdot\left\{A_{21} \cdot \varphi_{Q}^{T}+\left(q+\frac{\Delta \mathrm{q}}{n}+A_{21} \cdot\right.\right.\right. \\
\left.\left.Q_{0 i}\right)-A_{21} \cdot D_{11 \varphi}{ }^{-1} \cdot\left(A_{11 \varphi} \cdot \varphi_{Q}^{T}+\left(A_{10} \cdot H_{0}+A_{11 \vartheta} \cdot Q+A_{12} \cdot H\right)\right)\right\}- \\
\left.\left(A_{11 \varphi} \cdot \varphi_{Q}^{T}+\left(A_{10} \cdot H_{0}+A_{11 \vartheta} \cdot Q+A_{12} \cdot H\right)\right)\right\} \\
\varphi_{Q}^{T+1}=\varphi_{Q}^{T}+D_{11 \varphi}{ }^{-1} \cdot\left\{-A_{12} \cdot\left(A_{21} \cdot D_{11 \varphi}{ }^{-1} \cdot A_{12}\right)^{-1} \cdot\left\{A_{21} \cdot \varphi_{Q}^{T}+\left(q+\frac{\Delta \mathrm{q}}{n}+A_{21} \cdot\right.\right.\right. \\
\left.\left.Q_{0 i}\right)-A_{21} \cdot D_{11 \varphi}{ }^{-1} \cdot\left(A_{11 \varphi} \cdot \varphi_{Q}^{T}+\left(A_{10} \cdot H_{0}+A_{11 \vartheta} \cdot Q+A_{12} \cdot H\right)\right)\right\}- \\
\left.A_{11 \varphi} \cdot \varphi_{Q}^{T}-\left(A_{10} \cdot H_{0}+A_{11 \vartheta} \cdot Q+A_{12} \cdot H\right)\right\}
\end{gathered}
$$

A partir de este punto, el método del gradiente se convierte en un proceso iterativo con las expresiones 4.61 y 4.62 . En cada iteración, la solución aproximada $\left(\varphi_{Q}^{T}, \varphi_{H}^{T}\right)$ desprende en la solución $\left(\varphi_{Q}^{T+1}, \varphi_{H}^{T+1}\right)$ más cercana a la solución del problema. El proceso seguirá iterando hasta que ambas soluciones converjan, en dicho caso la solución $\left(\varphi_{Q}^{T+1}, \varphi_{H}^{T+1}\right)$ será la sensibilidad media de los elementos de la red ante un incremento de demanda conocida en cualquier punto de la red. 


\subsection{Cálculo de la ubicación de la fuga}

En el caso de producirse una fuga en un sistema de distribución, y suponiendo una magnitud y ubicación (nudo) conocidas, los caudales circulantes por las tuberías y las presiones en los nudos podrían calcularse fácilmente resolviendo el sistema de ecuaciones 4.3. Sin embargo, mientras estimar la magnitud de una fuga recientemente detectada a través de un balance hídrico es rápido y sencillo, no lo es tanto el determinar su ubicación.

Si se origina una fuga en la red cuya ubicación es desconocida, entonces se puede considerar un posible caudal de fuga (también desconocido) en cada nudo $q_{i_{f}}$. Así, a cada nudo se le asignará un caudal que resultará de la suma de su demanda habitual $q_{i}$ más el potencial caudal de fuga $q_{i_{f}}$ (Figura 4.3). Está claro que la mayoría de los flujos de fuga agregados serán cero, y solo aquellos (idealmente, solo uno) en el área cercana a la ubicación de la fuga diferirán de cero, pero generalmente no hay más información para aclarar esta pregunta.

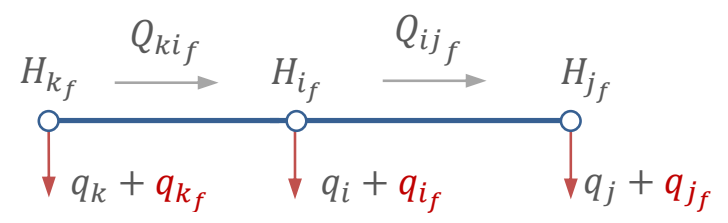

Figura 4.3. Red de distribución con hipotéticas fugas

Debido a la alteración que la fuga introduce en las condiciones de funcionamiento de la red, cada caudal de línea en el (nuevo) escenario de fuga, $Q_{i j_{f}}$, será diferente frente al valor (de referencia) en el escenario sin fuga, $Q_{i j_{0}}$.

Así, la ecuación de conservación de la masa para cada nudo i en el escenario con fugas será:

$$
\sum_{k=1}^{n_{i}} Q_{i k_{f}}+q_{i}+q_{i_{f}}=0
$$

donde $n_{i}$ es el número total de nudos conectados con el nudo $i, Q_{i k_{f}}$ es el nuevo caudal circulante por la tubería que conecta el nudo $i$ con el nudo $k, q_{i}$ es la demanda del nudo $i$ y $q_{i_{f}}$ es el caudal de fuga potencial en el nudo $i$.

Siguiendo con el procedimiento explicado con anterioridad, el sistema de las $n$ ecuaciones de conservación de la masa (una por cada nudo) puede expresarse como:

$$
\left[\begin{array}{lll}
A_{21} & \vdots & 1
\end{array}\right]\left[\begin{array}{c}
Q_{f} \\
\cdots \\
q_{f}
\end{array}\right]=-[q]
$$

donde $A_{21}$ y $q$ son los mismos términos de la ecuación $4.3, Q_{f}$ es el vector de los caudales de línea y $q_{f}$ es el vector de los valores de caudal fugado nodal. Tanto el vector $Q_{f}$ como el $q_{f}$ son valores desconocidos.

A su vez, la ecuación de conservación de la energía para cada tubería, la cual conecta el nudo $i$ con el nudo $j$, en el escenario con fuga es: 


$$
H_{i_{f}}-H_{j_{f}}=r_{i j} Q_{i j_{f}}\left|Q_{i j_{f}}\right|
$$

Las cuales pueden representarse, para toda la red, según el siguiente sistema:

$$
\left[\begin{array}{lll}
A_{11} & \vdots & A_{12}
\end{array}\right]\left[\begin{array}{c}
Q_{f} \\
\ldots \\
H_{f}
\end{array}\right]=-\left[A_{10} H_{0}\right]
$$

Donde $H_{f}$ es el vector de las alturas piezométricas en los nudos $H_{i_{f}}$, y $A_{11}, A_{10}$ y $H_{0}$ son los mismos términos de la ecuación 4.3.

Así, el nuevo sistema formado por las ecuaciones 4.64 y 4.66 , presenta $2 n+p$ factores desconocidos (que son las presiones y los caudales de fuga en los $n$ nudos y los caudales a través de las $p$ tuberías) y solo $n+p$ ecuaciones. Por razones obvias, este nuevo planteamiento no podrá resolverse. Para limitar el alcance del problema y encontrar una solución finita y real, se pueden introducir dos fuentes de información adicional: el análisis de sensibilidad y el uso de sensores para la toma de datos reales.

El primero aprovecha el análisis de la sensibilidad explicado anteriormente y propone la siguiente hipótesis: en una situación de fuga, la altura piezométrica de cualquier nudo puede aproximarse a la altura piezométrica en las condiciones de trabajo normales de ese nudo más su variación promedio calculada, $\left(H_{i_{f}}=H_{i}+\varphi_{H_{i}}\right)$, en base al incremento de demanda producido por la fuga en cualquier punto. Así, $n$ términos correspondientes a las alturas piezométricas en los nudos ahora se consideran conocidos, y el nuevo escenario planteado se puede resolver u obtener una aproximación a la solución real. La segunda fuente de información adicional son las mediciones reales de los caudalímetros y los sensores de presión instalados en tuberías y nudos de red específicos. Cualquiera que sea el número de dispositivos de cada tipo, las mediciones que proporcionan pueden organizarse en un vector $H_{D}$ para las alturas piezométricas de nudo conocidos, y un vector $Q_{D}$ para las tuberías de caudal conocidas.

Con ello, las ecuaciones de conservación de la masa 4.64 se pueden escribir según:

$$
\left[\begin{array}{lll}
A_{21} & \vdots & 1
\end{array}\right]\left[\begin{array}{c}
Q_{f} \\
\cdots \\
q_{f}
\end{array}\right]=-\left[A_{21} Q_{D}\right]-[q]
$$

Donde $Q_{D}$ es el vector de los caudales medidos por los caudalímetros en las tuberías (y cero en el resto de las tuberías) y el vector $Q_{f}$ adopta el valor cero en los valores correspondientes a las tuberías equipadas con caudalímetro.

A su vez, las ecuaciones de conservación de la energía 4.66 también pueden ser reescritas debido a que los valores de las alturas piezométricas en los nudos se consideran un valor conocido, y por lo tanto el vector $H_{f}$ se pasará a llamar $H_{D}$ :

$$
\left[A_{11} Q_{f}\right]=-\left[A_{11} Q_{f}\right]-\left[A_{12} H_{D}\right]-\left[A_{10} H_{0}\right]
$$

El sistema completo de ecuaciones de conservación para el escenario de fuga se puede obtener a partir de las ecuaciones 4.67 y 4.68 :

$$
\left[\begin{array}{ccc}
A_{11} & \vdots & 0 \\
\cdots & \cdots & \cdots \\
A_{21} & \vdots & 1
\end{array}\right]\left[\begin{array}{c}
Q_{f} \\
\cdots \\
q_{f}
\end{array}\right]=-\left[\begin{array}{ccc}
A_{11} & \vdots & A_{12} \\
\cdots & \cdots & \cdots \\
A_{21} & \vdots & 0
\end{array}\right]\left[\begin{array}{c}
Q_{D} \\
\cdots \\
H_{D}
\end{array}\right]-\left[\begin{array}{c}
A_{10} H_{0} \\
\cdots \\
q
\end{array}\right]
$$


donde $A_{11}, A_{12}, A_{21}, A_{10}, H_{0}$ y $q$ son los mismos términos de la ecuación $4.3, Q_{f}$ es el vector de los caudales de línea, $q_{f}$ es el vector de los valores de caudal fugado nodal, $Q_{D}$ es el vector de los caudales medidos por los caudalímetros en las tuberías (y cero en el resto de las tuberías), $H_{D}$ es el vector de las alturas piezométricas de los nudos (o bien monitorizadas por los sensores instalados en algunos de ellos, o bien aproximadas como $H_{i_{f}}=H_{i}+\varphi_{H_{i}}$ para el resto de los nudos).

Finalmente, el nuevo sistema 4.69 está compuesto por $n+p$ ecuaciones, y el número máximo de incógnitas sería $n+p$, para el caso en que no se instalen caudalímetros en la red. El número total de incógnitas se reduce según el número de caudalímetros instalados. Como puede observarse, este sistema de ecuaciones sí puede ser resuelto, aunque no de manera directa al tratarse de una ecuación no lineal. Nuevamente, se puede recurrir al método del gradiente para su resolución. Y tras realizar una expansión truncada de Taylor, se obtiene la siguiente estructura:

$$
\left[\begin{array}{ll}
D_{11}^{T} & 0 \\
A_{21} & I
\end{array}\right] \cdot\left[\begin{array}{l}
d Q_{f} \\
d q_{f}
\end{array}\right]=\left[\begin{array}{l}
-f_{1}^{T} \\
-f_{2}^{T}
\end{array}\right]
$$

Con:

$$
\begin{gathered}
f_{1}^{T}=A_{11} \cdot Q_{f}^{T}+A_{11} \cdot Q_{D}+A_{12} \cdot H_{D}+A_{10} \cdot H_{0} \\
f_{2}^{T}=A_{21} \cdot Q_{f}^{T}+q_{f}^{T}+A_{21} \cdot Q_{D}+q
\end{gathered}
$$

Desarrollando el sistema 4.70 se obtienen las siguientes dos ecuaciones:

$$
\begin{gathered}
D_{11}{ }^{T} \cdot d Q_{f}=-f_{1}^{T} \\
A_{21} \cdot d Q_{f}+d q_{f}=-f_{2}^{T}
\end{gathered}
$$

Se obtienen las ecuaciones para el cálculo del caudal de fuga y el caudal circulante no conocido.

$$
\begin{gathered}
d Q_{f}=Q_{f}^{T+1}-Q_{f}^{T} \\
d q_{f}=q_{f}^{T+1}-q_{f}^{T}
\end{gathered}
$$

Despejando $d Q_{f}$ de la ecuación 4.73 y operando con 4.71 , se obtendrá la ecuación para el cálculo del caudal de fuga.

$$
d Q_{f}=-D_{11}^{T}{ }^{-1}\left\{A_{11} \cdot Q_{f}^{T}+A_{11} \cdot Q_{D}+A_{12} \cdot H_{D}+A_{10} \cdot H_{0}\right\}
$$

Sustituyendo 4.77 en 4.74 :

$$
\begin{gathered}
-A_{21} \cdot D_{11}^{T}{ }^{-1}\left\{A_{11} \cdot Q_{f}^{T}+A_{11} \cdot Q_{D}+A_{12} \cdot H_{D}+A_{10} \cdot H_{0}\right\}+d q_{f}=-f_{2}^{T} \\
A_{21} \cdot D_{11}^{T}{ }^{-1}\left\{A_{11} \cdot Q_{f}^{T}+A_{11} \cdot Q_{D}+A_{12} \cdot H_{D}+A_{10} \cdot H_{0}\right\}-\left(q_{f}^{T+1}-q_{f}^{T}\right)=A_{21} \cdot Q_{f}^{T}+ \\
q_{f}^{T}+A_{21} \cdot Q_{D}+q
\end{gathered}
$$


Y operando:

$$
q_{f}^{T+1}=A_{21} \cdot\left\{-Q_{f}^{T}-Q_{D}+D_{11}^{T}-1 \cdot\left\{A_{11} \cdot Q_{f}^{T}+A_{11} \cdot Q_{D}+A_{12} \cdot H_{D}+A_{10} \cdot H_{0}\right\}\right\}-q
$$

Los caudales circulantes por las tuberías se obtendrán a partir de la ecuación 4.74, introduciendo $4.76,4.79,4.72$ y operando:

$$
\begin{gathered}
A_{21} \cdot d Q_{f}+d q_{f}=-f_{2}^{T} \\
A_{21} \cdot d Q_{f}+\left\{A_{21} \cdot\left\{-Q_{f}^{T}-Q_{D}+D_{11}^{T-1} \cdot\left\{A_{11} \cdot Q_{f}^{T}+A_{11} \cdot Q_{D}+A_{12} \cdot H_{D}+A_{10} \cdot H_{0}\right\}\right\}-q\right\}- \\
q_{f}^{T}=-\left\{A_{21} \cdot Q_{f}^{T}+q_{f}^{T}+A_{21} \cdot Q_{D}+q\right\} \\
A_{21} \cdot Q_{f}^{T+1}+A_{21} \cdot\left\{-Q_{f}^{T}-Q_{D}+D_{11}^{T-1} \cdot\left\{A_{11} \cdot Q_{f}^{T}+A_{11} \cdot Q_{D}+A_{12} \cdot H_{D}+A_{10} \cdot H_{0}\right\}\right\} \\
=-A_{21} \cdot Q_{D} \\
Q_{f}^{T+1}=Q_{f}^{T}-D_{11}^{T}-1 \cdot\left\{A_{11} \cdot Q_{f}^{T}+A_{11} \cdot Q_{D}+A_{12} \cdot H_{D}+A_{10} \cdot H_{0}\right\}
\end{gathered}
$$

A partir de este punto, el método del gradiente se convierte en un proceso iterativo con la expresión de 4.80. En cada iteración, la solución aproximada $\left(q_{f}^{T}, Q_{f}^{T}\right)$ desprende en la solución $\left(q_{f}^{T+1}, Q_{f}^{T+1}\right)$ más cercana a la solución del problema. El proceso seguirá iterando hasta que ambas soluciones converjan, en dicho caso la solución $\left(q_{f}^{T+1}, Q_{f}^{T+1}\right)$ será la sensibilidad media de los elementos de la red ante un incremento de demanda conocida en cualquier punto de la red.

\subsection{Ejemplo numérico}

Para una mejor compresión de la metodología desarrollada, ésta se aplicará sobre la red sintética mostrada en la Figura 4.4, usada como caso de estudio en el capítulo 3.

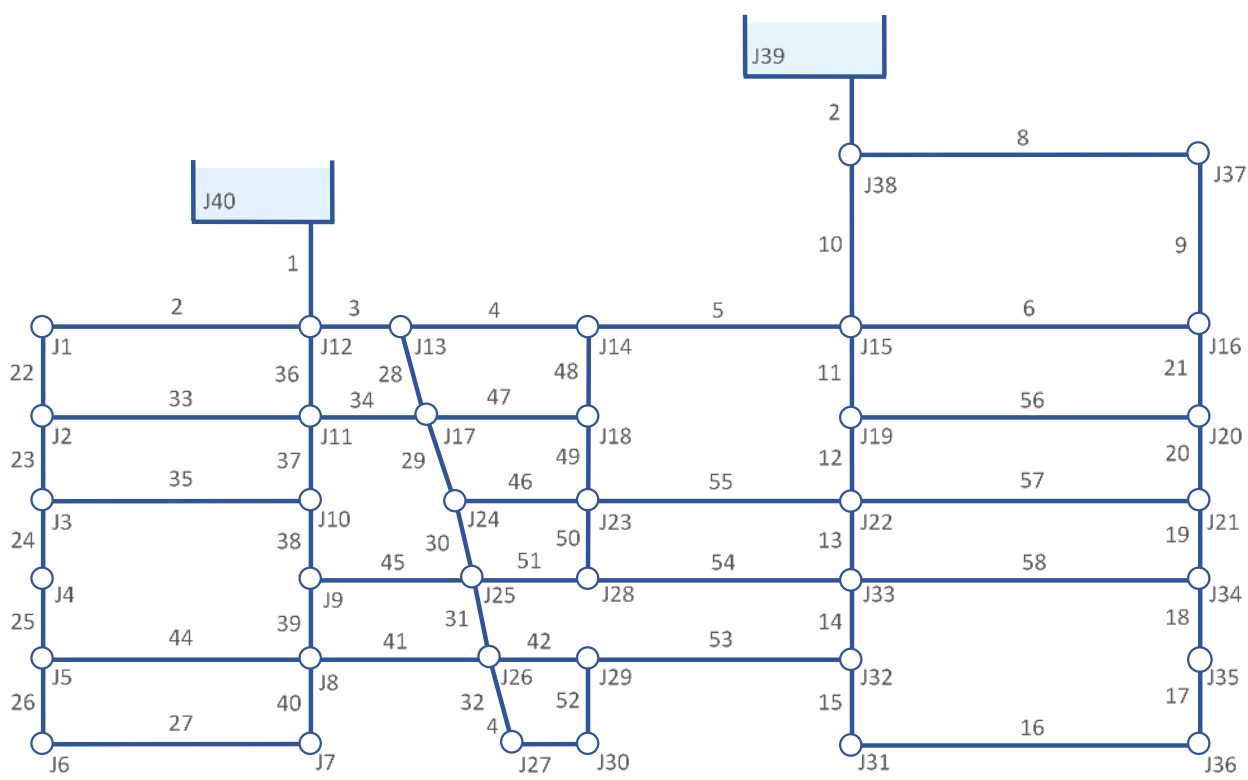

Figura 4.4. Diseño de la red de distribución. 
La demanda base es de $60 \mathrm{l} / \mathrm{min}\left(0,001 \mathrm{~m}^{3} / \mathrm{s}\right)$ para cada uno de los nudos entre el $1 \mathrm{y} 16, \mathrm{y}$ también para los nudos 21,27 y 30, mientras que, para los 19 nudos restantes, la demanda base es de $180 \mathrm{l} / \mathrm{min}\left(0,003 \mathrm{~m}^{3} / \mathrm{s}\right)$.

En su estado operativo normal, la presión promedio es de 37,7 mca, con valores extremos de 31,7 y 47,0 mca (Tabla 4.1). Los valores promedio de velocidad del agua y pérdida de carga unitaria en las tuberías son $0,41 \mathrm{~m} / \mathrm{s}$ y $2,50 \mathrm{mca} / \mathrm{km}$, respectivamente (Tabla 3.14). La procedencia del caudal es, aproximadamente, del $50 \%$ de cada depósito.

Para un análisis de la sensibilidad hidráulica de los elementos de la red, se han definido incrementos de 15, 30 y $60 \mathrm{l} /$ minuto en cada nudo. La razón de estos valores está en que la aparición de una fuga de $15 \circ 30 \mathrm{l} /$ minuto (o valores mayores) puede ser detectada en sectores monitoreados mediante análisis de flujo nocturno o balances hídricos. Además, estas cifras son del mismo orden de magnitud que las consideradas por Fuchs-Hanusch \& Steffelbauer (2017), en un estudio similar.

Como resultado para los tres escenarios, los resultados de las sensibilidades medias de los nudos se muestran en la Tabla 4.1. Como se esperaba, cuanto mayor es el aumento de la demanda, mayor es la disminución de la presión en los nodos (lógicamente, los que están más alejados de los puntos de entrada se ven más afectados). Por lo tanto, la disminución de presión promedio para los nodos de la red en general es $-0,2 \%$ para $15 \mathrm{I} /$ minuto, y $-0,4 \%$ y $0,9 \%$ para 30 y $60 \mathrm{l} /$ minuto, respectivamente.

Los resultados del análisis de la sensibilidad del caudal en las tuberías se muestran en la Tabla 4.2. Las magnitudes de los cambios de flujo promedio para cada aumento de la demanda (15, 30 y $60 \mathrm{l} /$ minuto) son $0,52 \%, 1,05 \%$ y $2,13 \%$, respectivamente. Esto da una mejor idea de la sensibilidad real y explica por qué las dos tuberías, 27 y 44, con variaciones de más del $20 \%$ tienen dos de los caudales de red más bajos (aproximadamente cien veces menos que el caudal promedio por tubería).

El análisis de sensibilidad hidráulica resalta aquellos nudos con mayores variaciones de presión (ya sea en términos totales o porcentuales): nodos 5, 6 y 25; así como aquellos cuyos cambios son mucho menos relevantes, a saber, los nodos 12, 15 y 34 . En paralelo, las tuberías más sensibles son la 8, 36 y 37 (sin tener en cuenta las que conectan cada depósito), mientras que las menos significativas son la 16, 43 y 46 .

Tabla 4.1. Análisis de la sensibilidad nodal

\begin{tabular}{|c|c|c|c|c|c|c|c|}
\hline \multirow{2}{*}{ Nudo ID } & \multirow{2}{*}{$\begin{array}{c}\text { Presión } \\
\text { inicial (mca) }\end{array}$} & \multicolumn{6}{|c|}{ Variación de la presión (mca) } \\
\cline { 3 - 9 } & & $\begin{array}{c}\text { Incremento de } 15 \\
\text { I/min }\end{array}$ & \multicolumn{2}{|c|}{$\begin{array}{c}\text { Incremento de } 30 \\
\text { I/min }\end{array}$} & \multicolumn{2}{|c|}{$\begin{array}{c}\text { Incremento de 60 } \\
\text { I/min }\end{array}$} \\
\hline 1 & 41,8 & $-0,1$ & $-0,2 \%$ & $-0,1$ & $-0,3 \%$ & $-0,3$ & $-0,6 \%$ \\
\hline 2 & 39,8 & $-0,1$ & $-0,2 \%$ & $-0,2$ & $-0,4 \%$ & $-0,3$ & $-0,9 \%$ \\
\hline 3 & 38,3 & $-0,1$ & $-0,3 \%$ & $-0,2$ & $-0,5 \%$ & $-0,4$ & $-1,0 \%$ \\
\hline 4 & 36,7 & $-0,1$ & $-0,3 \%$ & $-0,2$ & $-0,6 \%$ & $-0,5$ & $-1,2 \%$ \\
\hline 5 & 35,9 & $-0,1$ & $-0,3 \%$ & $-0,2$ & $-0,7 \%$ & $-0,5$ & $-1,3 \%$ \\
\hline 6 & 35,7 & $-0,1$ & $-0,3 \%$ & $-0,2$ & $-0,7 \%$ & $-0,5$ & $-1,4 \%$ \\
\hline 7 & 35,6 & $-0,1$ & $-0,3 \%$ & $-0,2$ & $-0,6 \%$ & $-0,4$ & $-1,2 \%$ \\
\hline 8 & 35,8 & $-0,1$ & $-0,3 \%$ & $-0,2$ & $-0,6 \%$ & $-0,4$ & $-1,2 \%$ \\
\hline 9 & 38,7 & $-0,1$ & $-0,2 \%$ & $-0,2$ & $-0,4 \%$ & $-0,3$ & $-0,8 \%$ \\
\hline
\end{tabular}




\begin{tabular}{|c|c|c|c|c|c|c|c|}
\hline \multirow{3}{*}{$\begin{array}{c}\text { Nudo ID } \\
10\end{array}$} & \multirow{3}{*}{$\begin{array}{c}\text { Presión } \\
\text { inicial (mca) } \\
\\
40,0\end{array}$} & \multicolumn{6}{|c|}{ Variación de la presión (mca) } \\
\hline & & \multicolumn{2}{|c|}{$\begin{array}{l}\text { Incremento de } 15 \\
\text { I/min }\end{array}$} & \multicolumn{2}{|c|}{$\begin{array}{c}\text { Incremento de } 30 \\
\text { I/min }\end{array}$} & \multicolumn{2}{|c|}{$\begin{array}{c}\text { Incremento de } 60 \\
\text { I/min }\end{array}$} \\
\hline & & $-0,1$ & $-0,2 \%$ & $-0,1$ & $-0,4 \%$ & $-0,3$ & $-0,7 \%$ \\
\hline 11 & 41,3 & $-0,1$ & $-0,2 \%$ & $-0,1$ & $-0,3 \%$ & $-0,3$ & $-0,6 \%$ \\
\hline 12 & 43,0 & $-0,1$ & $-0,1 \%$ & $-0,1$ & $-0,2 \%$ & $-0,2$ & $-0,5 \%$ \\
\hline 13 & 39,4 & $-0,1$ & $-0,2 \%$ & $-0,2$ & $-0,4 \%$ & $-0,3$ & $-0,8 \%$ \\
\hline 14 & 39,1 & $-0,1$ & $-0,2 \%$ & $-0,2$ & $-0,4 \%$ & $-0,3$ & $-0,8 \%$ \\
\hline 15 & 43,6 & 0,0 & $-0,1 \%$ & $-0,1$ & $-0,2 \%$ & $-0,2$ & $-0,4 \%$ \\
\hline 16 & 40,4 & $-0,1$ & $-0,2 \%$ & $-0,1$ & $-0,3 \%$ & $-0,3$ & $-0,6 \%$ \\
\hline 17 & 36,9 & $-0,1$ & $-0,2 \%$ & $-0,2$ & $-0,5 \%$ & $-0,4$ & $-1,0 \%$ \\
\hline 18 & 36,6 & $-0,1$ & $-0,3 \%$ & $-0,2$ & $-0,5 \%$ & $-0,4$ & $-1,0 \%$ \\
\hline 19 & 38,8 & $-0,1$ & $-0,2 \%$ & $-0,2$ & $-0,4 \%$ & $-0,3$ & $-0,8 \%$ \\
\hline 20 & 39,4 & $-0,1$ & $-0,2 \%$ & $-0,1$ & $-0,4 \%$ & $-0,3$ & $-0,7 \%$ \\
\hline 21 & 38,8 & $-0,1$ & $-0,2 \%$ & $-0,2$ & $-0,4 \%$ & $-0,3$ & $-0,9 \%$ \\
\hline 22 & 36,8 & $-0,1$ & $-0,3 \%$ & $-0,2$ & $-0,5 \%$ & $-0,4$ & $-1,1 \%$ \\
\hline 23 & 36,5 & $-0,1$ & $-0,3 \%$ & $-0,2$ & $-0,5 \%$ & $-0,5$ & $-1,2 \%$ \\
\hline 24 & 36,5 & $-0,1$ & $-0,3 \%$ & $-0,2$ & $-0,5 \%$ & $-0,5$ & $-1,3 \%$ \\
\hline 25 & 37,1 & $-0,1$ & $-0,3 \%$ & $-0,2$ & $-0,5 \%$ & $-0,5$ & $-1,3 \%$ \\
\hline 26 & 35,5 & $-0,1$ & $-0,3 \%$ & $-0,2$ & $-0,6 \%$ & $-0,4$ & $-1,2 \%$ \\
\hline 27 & 31,7 & $-0,1$ & $-0,3 \%$ & $-0,3$ & $-1,0 \%$ & $-0,4$ & $-1,3 \%$ \\
\hline 28 & 36,8 & $-0,1$ & $-0,2 \%$ & $-0,2$ & $-0,5 \%$ & $-0,3$ & $-0,9 \%$ \\
\hline 29 & 35,5 & $-0,1$ & $-0,2 \%$ & $-0,2$ & $-0,6 \%$ & $-0,3$ & $-0,8 \%$ \\
\hline 30 & 31,7 & $-0,1$ & $-0,2 \%$ & $-0,3$ & $-1,0 \%$ & $-0,3$ & $-0,8 \%$ \\
\hline 31 & 33,5 & $-0,1$ & $-0,2 \%$ & $-0,2$ & $-0,6 \%$ & $-0,2$ & $-0,6 \%$ \\
\hline 32 & 35,7 & $-0,1$ & $-0,2 \%$ & $-0,2$ & $-0,5 \%$ & $-0,3$ & $-0,8 \%$ \\
\hline 33 & 36,6 & $-0,1$ & $-0,2 \%$ & $-0,2$ & $-0,5 \%$ & $-0,3$ & $-0,9 \%$ \\
\hline 34 & 38,3 & 0,0 & $-0,1 \%$ & $-0,2$ & $-0,4 \%$ & $-0,2$ & $-0,4 \%$ \\
\hline 35 & 34,0 & $-0,1$ & $-0,2 \%$ & $-0,2$ & $-0,6 \%$ & $-0,3$ & $-0,7 \%$ \\
\hline 36 & 33,1 & $-0,1$ & $-0,3 \%$ & $-0,2$ & $-0,6 \%$ & $-0,4$ & $-1,1 \%$ \\
\hline 37 & 42,3 & $-0,1$ & $-0,2 \%$ & $-0,1$ & $-0,2 \%$ & $-0,4$ & $-0,8 \%$ \\
\hline 38 & 47,0 & $-0,1$ & $-0,2 \%$ & 0,0 & $-0,1 \%$ & $-0,3$ & $-0,6 \%$ \\
\hline
\end{tabular}

Tabla 4.2. Análisis de sensibilidad en tuberías

\begin{tabular}{|c|c|c|c|c|c|c|c|}
\hline \multirow{2}{*}{$\begin{array}{c}\text { Tubería } \\
\text { ID }\end{array}$} & \multirow{2}{*}{$\begin{array}{c}\text { Caudal } \\
\text { inicial } \\
\text { (I/min) }\end{array}$} & \multicolumn{5}{|c|}{ Variación del caudal (I/min) } \\
\cline { 3 - 8 } & Incremento & \multicolumn{2}{|c|}{$\begin{array}{c}\text { Incremento } \\
\text { de } 15 \text { I/min }\end{array}$} & \multicolumn{2}{|c|}{$\begin{array}{l}\text { Incremento } \\
\text { de } 30 \text { I/min }\end{array}$} & \multicolumn{2}{|c|}{ de $/$ min } \\
\hline 1 & 2298,3 & 8,0 & $0,4 \%$ & 16,0 & $0,7 \%$ & 32,0 & $1,4 \%$ \\
\hline 2 & 330,2 & 1,6 & $0,5 \%$ & 3,3 & $1,0 \%$ & 6,5 & $2,0 \%$ \\
\hline 3 & 345,4 & 1,1 & $0,3 \%$ & 2,1 & $0,6 \%$ & 4,2 & $1,2 \%$ \\
\hline 4 & 68,1 & 0,2 & $0,2 \%$ & 0,3 & $0,5 \%$ & 0,6 & $0,9 \%$ \\
\hline 5 & 218,8 & 0,8 & $0,4 \%$ & 1,5 & $0,7 \%$ & 3,1 & $1,4 \%$ \\
\hline
\end{tabular}

\begin{tabular}{|c|c|c|c|c|c|c|c|}
\hline \multirow{2}{*}{$\begin{array}{c}\text { Tubería } \\
\text { ID }\end{array}$} & $\begin{array}{c}\text { Caudal } \\
\text { inicial } \\
\text { (I/min) }\end{array}$ & \multicolumn{5}{|c|}{ Variación del caudal (I/min) } \\
\cline { 3 - 8 } & Incremento & \multicolumn{2}{|c|}{$\begin{array}{c}\text { Incremento } \\
\text { de 30 I/min }\end{array}$} & \multicolumn{2}{|c|}{$\begin{array}{c}\text { Incremento } \\
\text { de 60 I/min }\end{array}$} \\
\hline 30 & 105,8 & 0,2 & $0,2 \%$ & 0,4 & $0,3 \%$ & 0,7 & $0,7 \%$ \\
\hline 31 & 194,8 & 0,8 & $0,4 \%$ & 1,6 & $0,8 \%$ & 3,1 & $1,6 \%$ \\
\hline 32 & 60,9 & 0,4 & $0,7 \%$ & 0,8 & $1,3 \%$ & 1,6 & $2,6 \%$ \\
\hline 33 & 18,7 & 0,1 & $0,7 \%$ & 0,3 & $1,3 \%$ & 0,5 & $2,7 \%$ \\
\hline 34 & 50,9 & 0,1 & $0,3 \%$ & 0,3 & $0,5 \%$ & 0,5 & $1,0 \%$ \\
\hline
\end{tabular}




\begin{tabular}{|c|c|c|c|c|c|c|c|}
\hline \multirow{3}{*}{$\begin{array}{c}\text { Tubería } \\
\text { ID } \\
6\end{array}$} & \multirow{3}{*}{$\begin{array}{c}\begin{array}{c}\text { Caudal } \\
\text { inicial } \\
\text { (1/min) }\end{array} \\
25,9\end{array}$} & \multicolumn{6}{|c|}{ Variación del caudal (I/min) } \\
\hline & & \multicolumn{2}{|c|}{$\begin{array}{l}\text { Incremento } \\
\text { de } 15 \mathrm{l} / \mathrm{min}\end{array}$} & \multicolumn{2}{|c|}{$\begin{array}{l}\text { Incremento } \\
\text { de } 30 \mathrm{l} / \mathrm{min}\end{array}$} & \multicolumn{2}{|c|}{$\begin{array}{l}\text { Incremento } \\
\text { de } 60 \mathrm{l} / \mathrm{min}\end{array}$} \\
\hline & & 0,1 & $0,3 \%$ & 0,2 & $0,6 \%$ & 0,3 & $1,2 \%$ \\
\hline 7 & 2261,7 & 7,0 & $0,3 \%$ & 14,0 & $0,6 \%$ & 28,0 & $1,2 \%$ \\
\hline 8 & 1341,3 & 4,1 & $0,3 \%$ & 8,2 & $0,6 \%$ & 16,4 & $1,2 \%$ \\
\hline 9 & 1161,3 & 3,7 & $0,3 \%$ & 7,4 & $0,6 \%$ & 14,9 & $1,3 \%$ \\
\hline 10 & 740,5 & 2,5 & $0,3 \%$ & 5,0 & $0,7 \%$ & 9,9 & $1,3 \%$ \\
\hline 11 & 435,8 & 1,3 & $0,3 \%$ & 2,5 & $0,6 \%$ & 5,0 & $1,1 \%$ \\
\hline 12 & 266,5 & 0,9 & $0,3 \%$ & 1,8 & $0,7 \%$ & 3,6 & $1,3 \%$ \\
\hline 13 & 70,2 & 0,4 & $0,6 \%$ & 0,9 & $1,2 \%$ & 1,8 & $2,5 \%$ \\
\hline 14 & 531,6 & 1,9 & $0,4 \%$ & 3,8 & $0,7 \%$ & 7,7 & $1,4 \%$ \\
\hline 15 & 222,0 & 0,4 & $0,2 \%$ & 0,8 & $0,4 \%$ & 1,6 & $0,7 \%$ \\
\hline 16 & 42,0 & 0,0 & $0,0 \%$ & 0,0 & $0,0 \%$ & 0,0 & $0,0 \%$ \\
\hline 17 & 138,0 & 0,4 & $0,3 \%$ & 0,8 & $0,6 \%$ & 1,6 & $1,1 \%$ \\
\hline 18 & 318,0 & 0,8 & $0,3 \%$ & 1,6 & $0,5 \%$ & 3,2 & $1,0 \%$ \\
\hline 19 & 856,0 & 2,5 & $0,3 \%$ & 5,0 & $0,6 \%$ & 10,0 & $1,2 \%$ \\
\hline 20 & 936,4 & 3,0 & $0,3 \%$ & 5,9 & $0,6 \%$ & 11,9 & $1,3 \%$ \\
\hline 21 & 1127,1 & 3,4 & $0,3 \%$ & 6,8 & $0,6 \%$ & 13,6 & $1,2 \%$ \\
\hline 22 & 270,2 & 1,2 & $0,5 \%$ & 2,5 & $0,9 \%$ & 5,0 & $1,8 \%$ \\
\hline 23 & 228,9 & 1,0 & $0,4 \%$ & 1,9 & $0,9 \%$ & 3,9 & $1,7 \%$ \\
\hline 24 & 188,1 & 0,7 & $0,4 \%$ & 1,5 & $0,8 \%$ & 2,9 & $1,5 \%$ \\
\hline 25 & 128,1 & 0,3 & $0,3 \%$ & 0,7 & $0,5 \%$ & 1,3 & $1,0 \%$ \\
\hline 26 & 63,7 & 0,2 & $0,3 \%$ & 0,4 & $0,6 \%$ & 0,7 & $1,1 \%$ \\
\hline 27 & 3,7 & $-0,2$ & $-5,6 \%$ & $-0,4$ & $11,5 \%$ & $-0,9$ & $23,0 \%$ \\
\hline 28 & 217,3 & 0,5 & $0,2 \%$ & 1,0 & $0,5 \%$ & 2,0 & $0,9 \%$ \\
\hline 29 & 79,0 & 0,2 & $0,3 \%$ & 0,4 & $0,5 \%$ & 0,9 & $1,1 \%$ \\
\hline
\end{tabular}

\begin{tabular}{|c|c|c|c|c|c|c|c|}
\hline \multirow{3}{*}{$\begin{array}{c}\begin{array}{c}\text { Tubería } \\
\text { ID }\end{array} \\
35\end{array}$} & \multirow{3}{*}{$\begin{array}{c}\begin{array}{c}\text { Caudal } \\
\text { inicial } \\
\text { (I/min) }\end{array} \\
19,2\end{array}$} & \multicolumn{6}{|c|}{ Variación del caudal (I/min) } \\
\hline & & \multicolumn{2}{|c|}{$\begin{array}{l}\text { Incremento } \\
\text { de } 15 \mathrm{l} / \mathrm{min}\end{array}$} & \multicolumn{2}{|c|}{$\begin{array}{l}\text { Incremento } \\
\text { de } 30 \mathrm{l} / \mathrm{min}\end{array}$} & \multicolumn{2}{|c|}{$\begin{array}{l}\text { Incremento } \\
\text { de } 60 \mathrm{l} / \mathrm{min}\end{array}$} \\
\hline & & 0,2 & $0,8 \%$ & 0,3 & $1,5 \%$ & 0,6 & $3,0 \%$ \\
\hline 36 & 1562,7 & 4,9 & $0,3 \%$ & 9,9 & $0,6 \%$ & 19,7 & $1,3 \%$ \\
\hline 37 & 1433,1 & 4,3 & $0,3 \%$ & 8,6 & $0,6 \%$ & 17,1 & $1,2 \%$ \\
\hline 38 & 1353,9 & 3,7 & $0,3 \%$ & 7,5 & $0,6 \%$ & 15,0 & $1,1 \%$ \\
\hline 39 & 267,5 & 0,9 & $0,4 \%$ & 1,9 & $0,7 \%$ & 3,8 & $1,4 \%$ \\
\hline 40 & 56,3 & 0,6 & $1,1 \%$ & 1,2 & $2,2 \%$ & 2,4 & $4,3 \%$ \\
\hline 41 & 155,6 & $-0,3$ & $-0,2 \%$ & $-0,6$ & $-0,4 \%$ & $-1,3$ & $-0,8 \%$ \\
\hline 42 & 109,5 & $-0,3$ & $-0,3 \%$ & $-0,7$ & $-0,6 \%$ & $-1,3$ & $-1,2 \%$ \\
\hline 43 & 0,9 & 0,0 & $0,0 \%$ & 0,0 & $0,0 \%$ & 0,0 & $1,1 \%$ \\
\hline 44 & 4,3 & $-0,2$ & $-5,5 \%$ & $-0,5$ & $-11,3 \%$ & $-1,0$ & $-23,3 \%$ \\
\hline 45 & 1026,4 & 2,4 & $0,2 \%$ & 4,8 & $0,5 \%$ & 9,6 & $0,9 \%$ \\
\hline 46 & 4,8 & 0,0 & $0,0 \%$ & 0,0 & $0,2 \%$ & 0,0 & $0,2 \%$ \\
\hline 47 & 9,2 & 0,0 & $0,2 \%$ & 0,0 & $0,4 \%$ & 0,1 & $1,0 \%$ \\
\hline 48 & 226,9 & 0,5 & $0,2 \%$ & 1,1 & $0,5 \%$ & 2,1 & $0,9 \%$ \\
\hline 49 & 56,1 & 0,2 & $0,3 \%$ & 0,3 & $0,5 \%$ & 0,6 & $1,1 \%$ \\
\hline 50 & 82,4 & 0,1 & $0,1 \%$ & 0,2 & $0,3 \%$ & 0,5 & $0,6 \%$ \\
\hline 51 & 545,8 & 1,1 & $0,2 \%$ & 2,1 & $0,4 \%$ & 4,2 & $0,8 \%$ \\
\hline 52 & 59,1 & 0,4 & $0,7 \%$ & 0,8 & $1,3 \%$ & 1,6 & $2,7 \%$ \\
\hline 53 & 129,6 & 1,1 & $0,9 \%$ & 2,2 & $1,7 \%$ & 4,5 & $3,5 \%$ \\
\hline 54 & 283,5 & 0,6 & $0,2 \%$ & 1,1 & $0,4 \%$ & 2,2 & $0,8 \%$ \\
\hline 55 & 36,8 & 0,1 & $0,4 \%$ & 0,3 & $0,7 \%$ & 0,5 & $1,4 \%$ \\
\hline 56 & 10,7 & 0,0 & $0,3 \%$ & 0,1 & $0,7 \%$ & 0,1 & $1,3 \%$ \\
\hline 57 & 20,4 & 0,1 & $0,3 \%$ & 0,1 & $0,7 \%$ & 0,3 & $1,4 \%$ \\
\hline 58 & 358,0 & 1,3 & $0,4 \%$ & 2,6 & $0,7 \%$ & 5,3 & $1,5 \%$ \\
\hline
\end{tabular}

Una vez completado el análisis de sensibilidad, se aborda el problema de la localización de fugas. Con fines demostrativos, se supone que son cinco los sensores de presión y cinco los caudalímetros que están disponibles para su instalación en la red. El criterio principal para la selección de los elementos en los que instalar ambos tipos de dispositivos es el de mayor sensibilidad. Un segundo criterio propondrá una distribución espacial razonable de los sensores. En consecuencia, los sensores de presión se instalan en los nodos 5, 18, 27, 30 y 37 ; y los medidores de flujo en las tuberías 1, 9, 20, 36 y 45. Es decir, se monitorea el 13\% de los nodos y el $9 \%$ de las tuberías.

La aplicación y los resultados del método de localización de fugas se demuestran de la siguiente manera: se supondrá, como cada caso es diferente, que se ha producido una fuga de $30 \mathrm{l} /$ minuto en cada uno de los nudos de la red. Es decir, se resolverán 38 casos diferentes. Para cada uno, además de saber en qué nodo se ubica la fuga, se conocerán los cinco valores de presión real y los cinco valores de caudal proporcionados, respectivamente, por los sensores de presión y los medidores de flujo instalados en la red (por simulación directa del caso). Estos diez elementos de datos se transferirán al modelo en el que se prueba el método, y cuya información se completa para el resto de los nodos con la carga promedio afectada por 
la sensibilidad promedio para un aumento en la demanda de $30 \mathrm{l} /$ minuto. Finalmente, y a través de la resolución del sistema de ecuaciones 4.69 , se obtiene el flujo de fuga para cada nodo (así como los flujos de la tubería de circulación). Los resultados se consideran exitosos si el caudal de fuga calculado es de aproximadamente $30 \mathrm{l} /$ minuto para el nudo con fuga 0 para los nudos vecinos, y es insignificante para los otros nudos de la red.

La Tabla 4.4 muestra el caudal de fuga obtenido para cada nudo (filas), medido en I/minuto, para cada una de las simulaciones (columnas). La tabla muestra que la metodología ha tenido éxito en el $63 \%$ de los casos ( 24 de las 38 simulaciones), ya que en 8 casos el nodo con fuga se identificó exactamente (en verde), y en otros 16 casos se identificó el área (nodo adyacente al nodo con la fuga, en amarillo). A partir de los datos en la Tabla 4.4, podemos observar que la ubicación precisa del nudo, o el área donde se está produciendo la fuga no controlada, depende en cierta medida de la existencia de un sensor de flujo o presión cercano. También es importante destacar la existencia de nudos con un consumo negativo (como en el caso del nodo 36 para la mayoría de las simulaciones). Es evidente que algunos nodos pueden necesitar una entrada de flujo para contrarrestar las desviaciones introducidas al estimar las alturas piezométricas desconocidas.

Como se esperaba, la ubicación de la fuga se muestra con menos precisión si en lugar de elegir elementos con una gran variación en la presión y el flujo, se eligen aquellos con una variación promedio más baja. Por ejemplo, si se seleccionaran los nodos 1, 12, 15, 16 y 38, y las tuberías $6,16,43,46$ y 56 , entonces menos del $50 \%$ de los casos (16 de los 38) serían exitosos (Tabla 4.5). Si los criterios de sensibilidad se dejan de lado y solo se consideran los criterios de distribución espacial, los resultados muestran una mayor variabilidad. Así, si los nodos 2, 8, 18, 20 y 32; y las tuberías 11, 17, 25, 36 y 42 fueran monitoreados, la tasa de éxito sería exactamente del $50 \%$ (Tabla 4.6 ).

Lógicamente, cuantos más sensores estén instalados en la red, mejores serán los resultados. Juntamente con los tres escenarios abordados, entre las Tabla 4.4 y la Tabla 4.10 se resuelven hasta siete casos con diferentes configuraciones de sensores, y se alcanza una tasa de éxito del $87 \%$ con 15 sensores de presión y 5 medidores de flujo. A modo de resumen, la Tabla 4.3 muestra los resultados detallados.

Tabla 4.3. Tabla resumen para las diversas configuraciones de sensores probadas en el estudio de caso

\begin{tabular}{|c|c|c|c|c|c|c|c|}
\hline Configuración & 1 & 2 & 3 & 4 & 5 & 6 & 7 \\
\hline № se sensores de presión & 5 & 5 & 5 & 10 & 10 & 15 & 15 \\
\hline № de caudalímetros & 5 & 5 & 5 & 0 & 5 & 0 & 5 \\
\hline Número escenarios & 38 & 38 & 38 & 38 & 38 & 38 & 38 \\
\hline $\begin{array}{c}\text { Ubicación de la fuga en el } \\
\text { nudo exacto }\end{array}$ & 8 & 8 & 9 & 10 & 10 & 15 & 16 \\
\hline $\begin{array}{c}\text { Detección del área } \\
\text { No detección }\end{array}$ & 16 & 8 & 10 & 10 & 13 & 15 & 17 \\
\hline Casos exitosos & 24 & 16 & 19 & 20 & 23 & 30 & 33 \\
\hline \% de casos exitosos & $63 \%$ & $42 \%$ & $50 \%$ & $53 \%$ & $61 \%$ & $79 \%$ & $87 \%$ \\
\hline
\end{tabular}


Tabla 4.4. Caudales de fuga obtenidos con 5 sensores de presión y 5 medidores de caudal instalados mediante la combinación de criterios de máxima sensibilidad y distribución espacial (nudos 5, 18, 27, 30, 37 y tuberías 1, 9, 20, 36, 45)

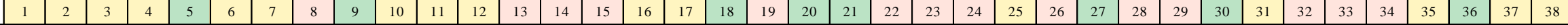

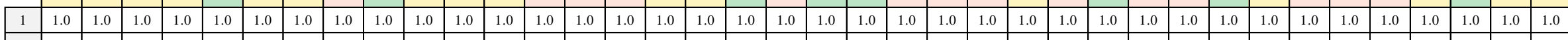

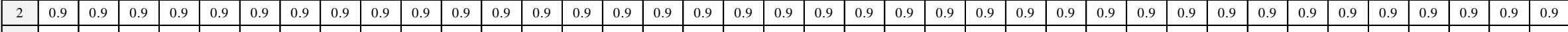

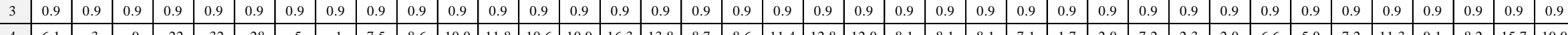

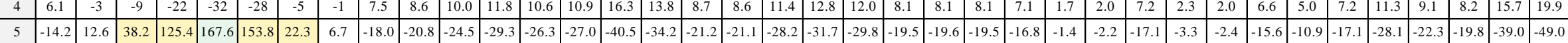

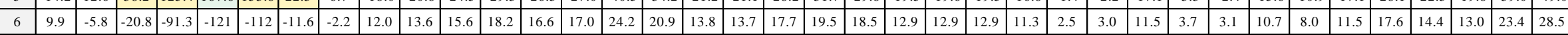

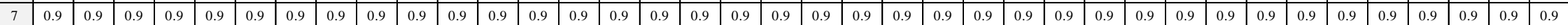

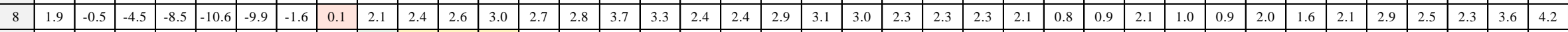

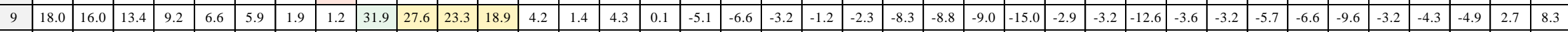

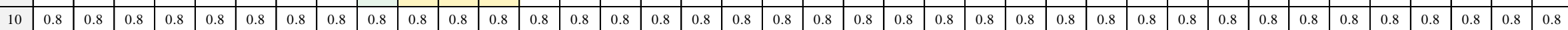

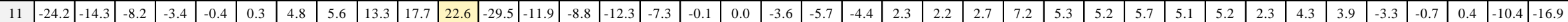

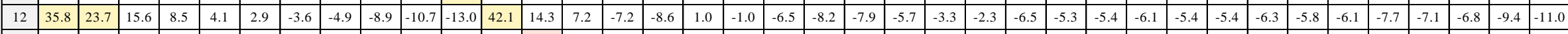

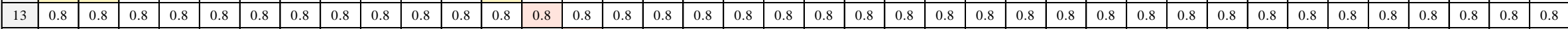

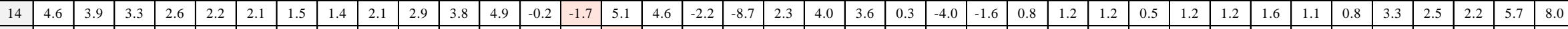

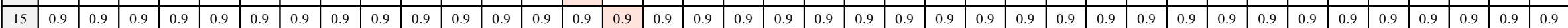

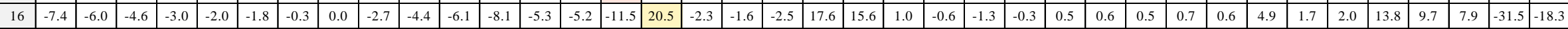

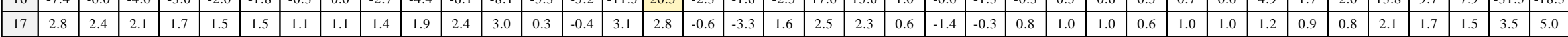

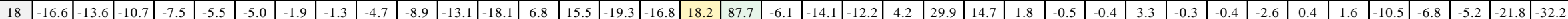
\begin{tabular}{lllllllllllllllllllllllllllllllllllllllllllllllll}
\hline 19 & 0.8 & 0.8 & 0.8 & 0.8 & 0.8 & 0.8 & 0.8 & 0.8 & 0.8 & 0.8 & 0.8 & 0.8 & 0.8 & 0.8 & 0.8 & 0.8 & 0.8 & 0.8 & 0.8 & 0.8 & 0.8 & 0.8 & 0.8 & 0.8 & 0.8 & 0.8 & 0.8 & 0.8 & 0.8 & 0.8 & 0.8 & 0.8 & 0.8 & 0.8 & 0.8 & 0.8 & 0.8 & 0.8 \\
\hline
\end{tabular}

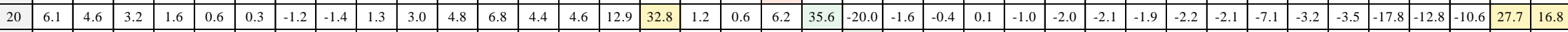

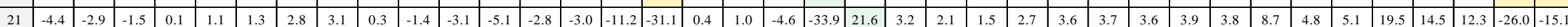

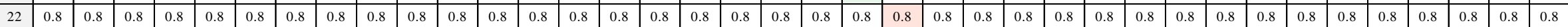

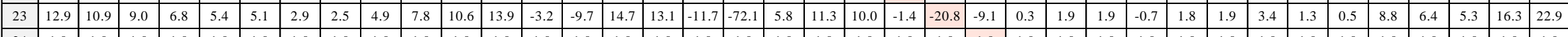

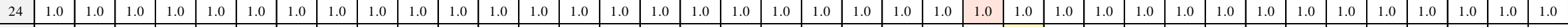

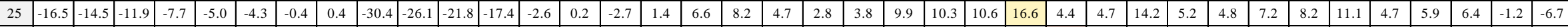

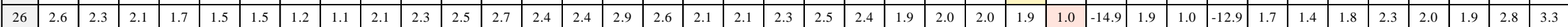

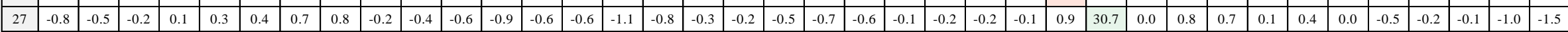

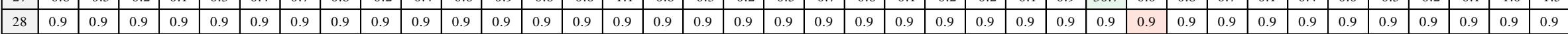

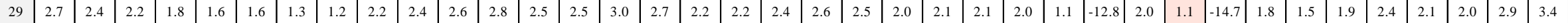

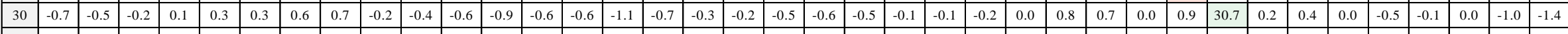

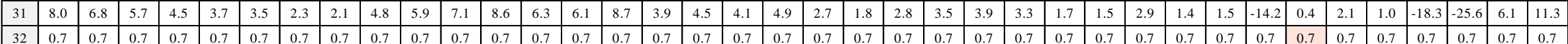

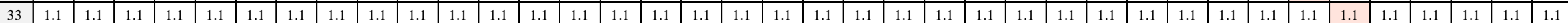
\begin{tabular}{|l|l|l|l|l|l|l|l|l|l|l|l|l|l|l|l|l|l|l|l|l|l|l|l|l|l|l|l|l|l|l|l|l|l|l|l|l|l|l}
\hline 34 & 0.9 & 0.9 & 0.9 & 0.9 & 0.9 & 0.9 & 0.9 & 0.9 & 0.9 & 0.9 & 0.9 & 0.9 & 0.9 & 0.9 & 0.9 & 0.9 & 0.9 & 0.9 & 0.9 & 0.9 & 0.9 & 0.9 & 0.9 & 0.9 & 0.9 & 0.9 & 0.9 & 0.9 & 0.9 & 0.9 & 0.9 & 0.9 & 0.9 & 0.9 & 0.9 & 0.9 & 0.9 & 0.9 \\
\hline
\end{tabular}

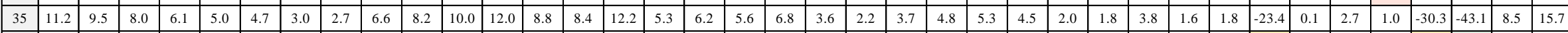

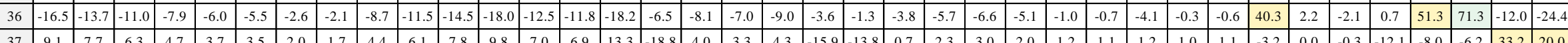

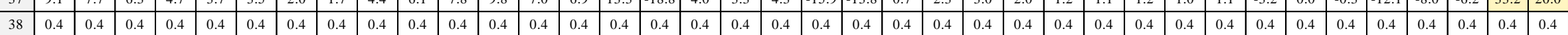


Tabla 4.5. Caudales de fuga obtenidos con 5 sensores de presión y 5 caudalímetros, instalados en los elementos menos sensibles de la red (nudos $1,12,15,16,38$ y tuberías $6,16,43,46,56$

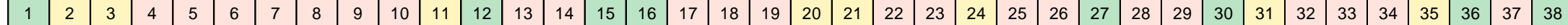

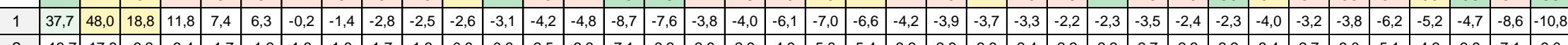

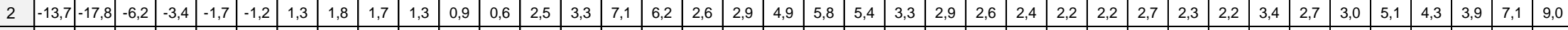

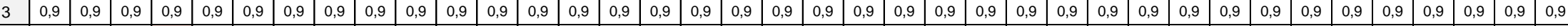

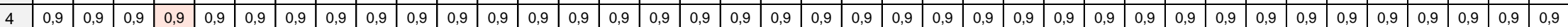

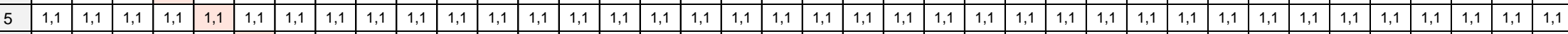

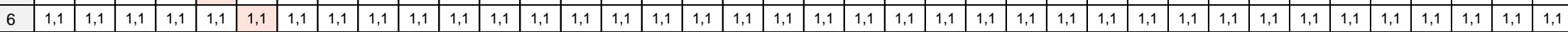

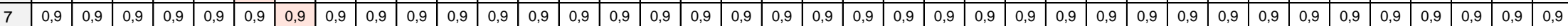
\begin{tabular}{llllllllllllllllllllllllllllllllllllllllllllllllll}
\hline 8 & 0,6 & 0,6 & 0,6 & 0,6 & 0,6 & 0,6 & 0,6 & 0,6 & 0,6 & 0,6 & 0,6 & 0,6 & 0,6 & 0,6 & 0,6 & 0,6 & 0,6 & 0,6 & 0,6 & 0,6 & 0,6 & 0,6 & 0,6 & 0,6 & 0,6 & 0,6 & 0,6 & 0,6 & 0,6 & 0,6 & 0,6 & 0,6 & 0,6 & 0,6 & 0,6 & 0,6 & 0,6 & 0,6 \\
\hline
\end{tabular}

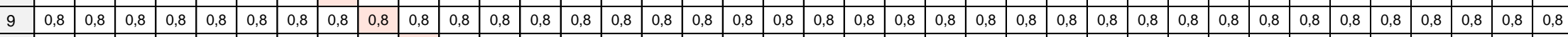

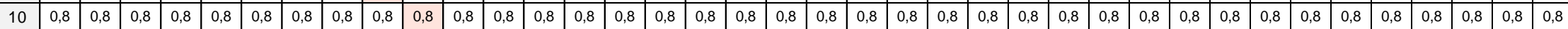
\begin{tabular}{|l|l|l|l|l|l|l|l|l|l|l|l|l|l|l|l|l|l|l|l|l|l|l|l|l|l|l|l|l|l|l|l|l|l|l|l|l|l|l|}
\hline 11 & $-14,5$ & $-17,5$ & $-8,5$ & $-5,1$ & $-3,0$ & $-2,5$ & 0,7 & 1,3 & $-4,0$ & $-7,7$ & $-11,6$ & $-16,1$ & $-1,2$ & 4,5 & 30,0 & 25,0 & 1,0 & 3,7 & 16,7 & 22,1 & 20,0 & 7,2 & 3,9 & 1,7 & 1,3 & 2,3 & 2,5 & 3,0 & 2,7 & 2,5 & 8,1 & 4,4 & 5,5 & 18,1 & 13,4 & 11,4 & 30,4 & 41,9 \\
\hline
\end{tabular}

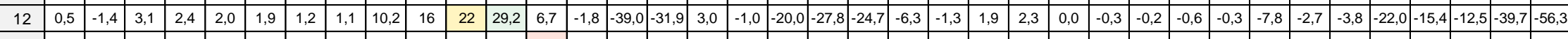

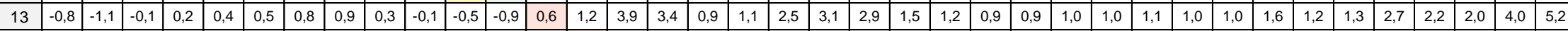

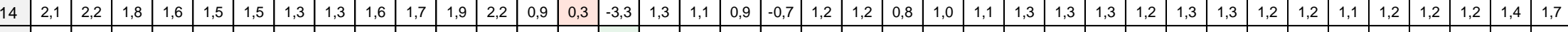

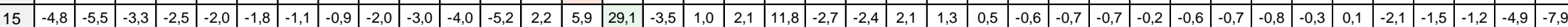

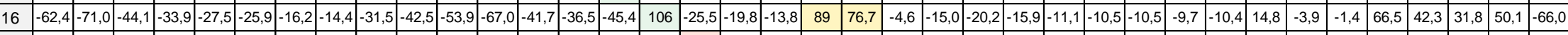

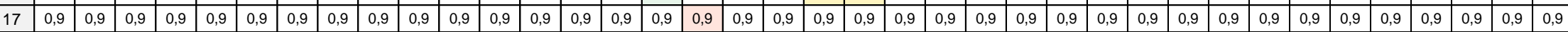

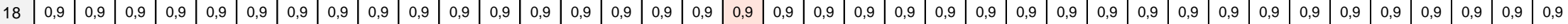

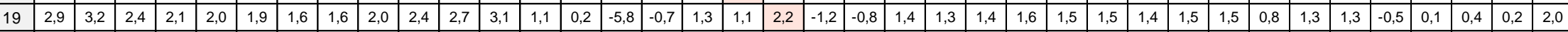

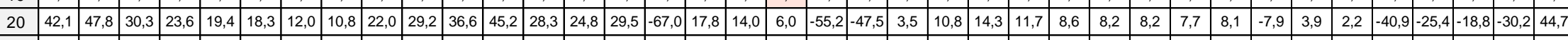
\begin{tabular}{|l|l|l|l|l|l|l|l|l|l|l|l|l|l|l|l|l|l|l|l|l|l|l|l|l|l|l|l|l|l|l|l|l|l|l|l|l|l|l|}
\hline 21 & 0,9 & 0,9 & 0,9 & 0,9 & 0,9 & 0,9 & 0,9 & 0,9 & 0,9 & 0,9 & 0,9 & 0,9 & 0,9 & 0,9 & 0,9 & 0,9 & 0,9 & 0,9 & 0,9 & 0,9 & 0,9 & 0,9 & 0,9 & 0,9 & 0,9 & 0,9 & 0,9 & 0,9 & 0,9 & 0,9 & 0,9 & 0,9 & 0,9 & 0,9 & 0,9 & 0,9 & 0,9 & 0,9 \\
\hline
\end{tabular}

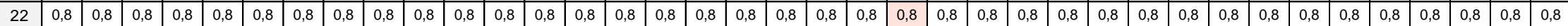

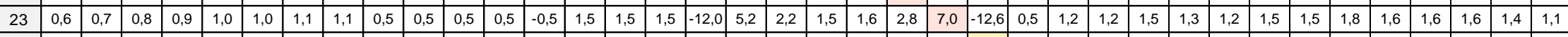

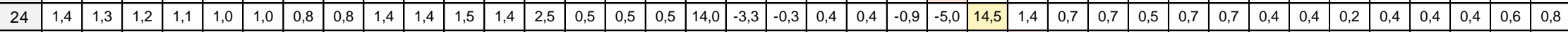

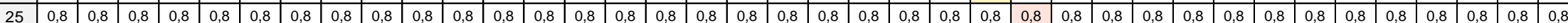

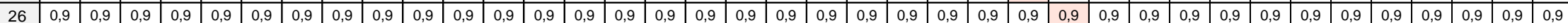

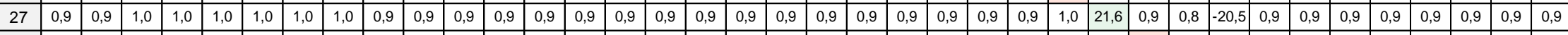
\begin{tabular}{|l|l|l|l|l|l|l|l|l|l|l|l|l|l|l|l|l|l|l|l|l|l|l|l|l|l|l|l|l|l}
\hline $28,9,9$ & 0,9 & 0,9 & 0,9 & 0,9 & 0,9 & 0,9 & 0,9 & 0,9 & 0,9 & 0,9 & 0,9 & 0,9 & 0,9 & 0,9 & 0,9 & 0,9 & 0,9 & 0,9 & 0,9 & 0,9 & 0,9 & 0,9 & 0,9 \\
\hline
\end{tabular}

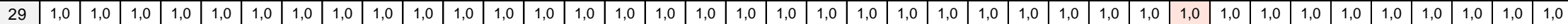

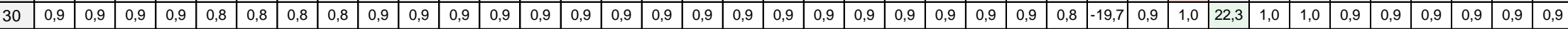

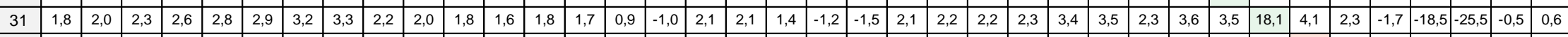

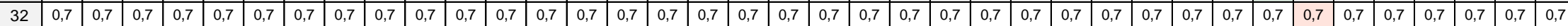

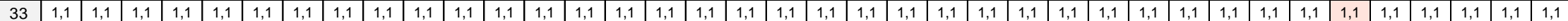

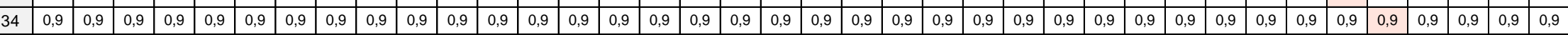

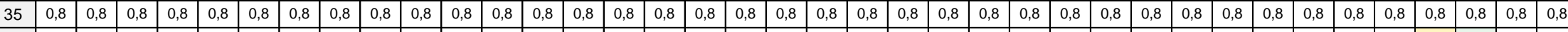

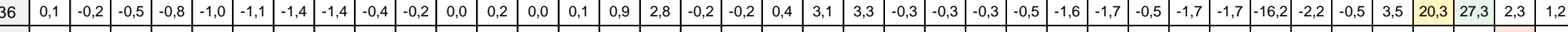

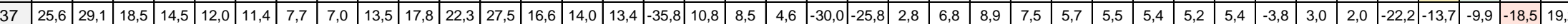

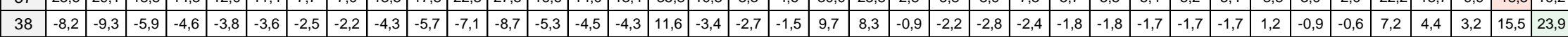


Tabla 4.6. Caudales de fuga obtenidos con 5 sensores de presión y 5 medidores de flujo, instalados de acuerdo con los criterios de distribución espacial (nudos $2,8,18,20,32$ y tuberías $11,17,25,36,42)$

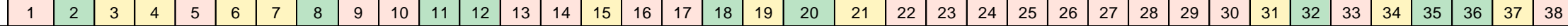

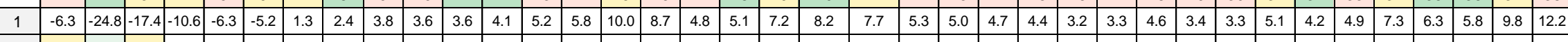

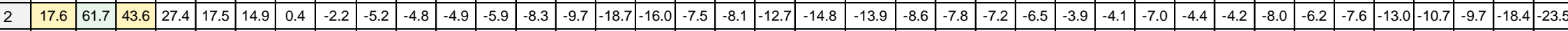

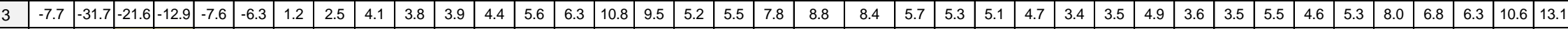

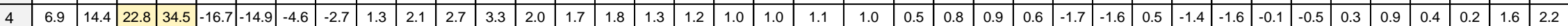

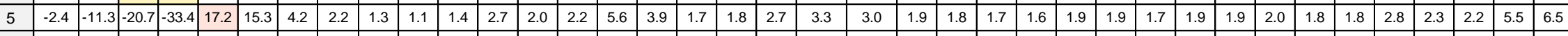

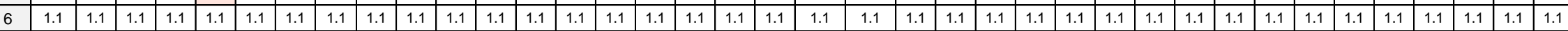

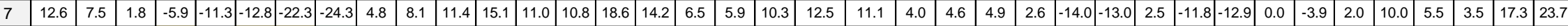

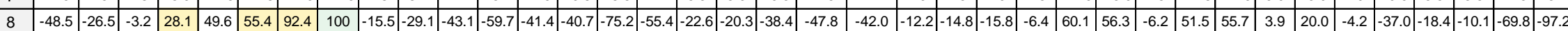
\begin{tabular}{l|l|l|l|l|l|l|l|l|l|l|l|l|l|l|l|l|l|l|l|l|l|l|l|l|l|l|l|l|l|l|l|l|l|lllll}
\hline 9 & 4.8 & 2.9 & 1.1 & -1.2 & -2.7 & -3.0 & -5.1 & -5.5 & 2.0 & 3.1 & 4.3 & 5.7 & 4.2 & 4.1 & 7.0 & 5.3 & 2.6 & 2.4 & 3.9 & 4.7 & 4.2 & 1.8 & 2.0 & 2.1 & 1.3 & -3.3 & -3.1 & 1.3 & -2.8 & -3.0 & 0.5 & -0.6 & 1.2 & 3.8 & 2.3 & 1.6 & 6.5 & 9.1 \\
\hline
\end{tabular}

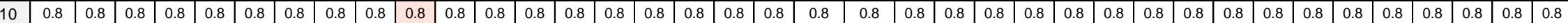

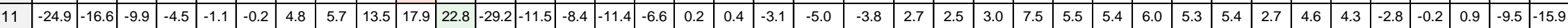

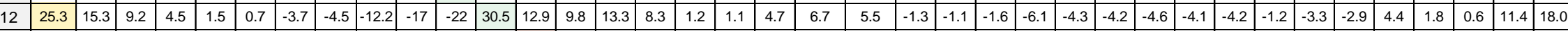

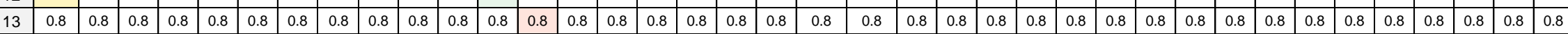

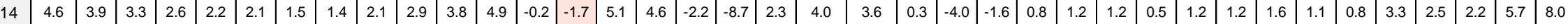

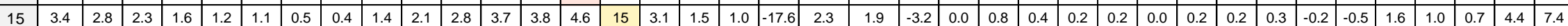

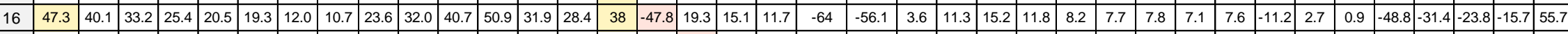

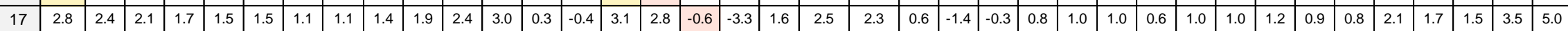

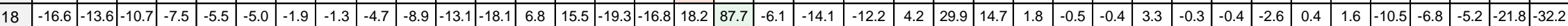

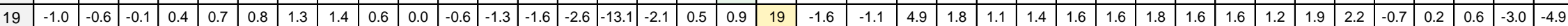

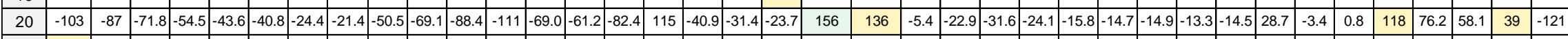

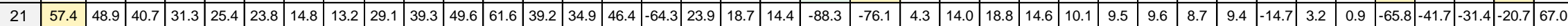

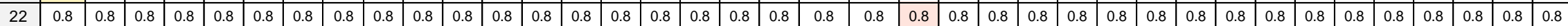

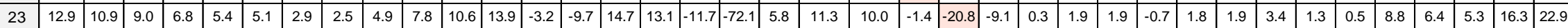

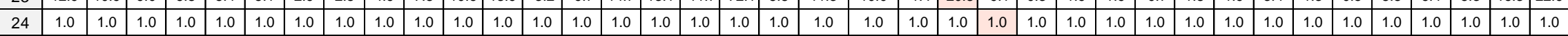

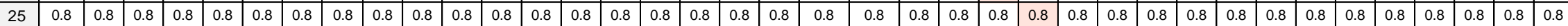

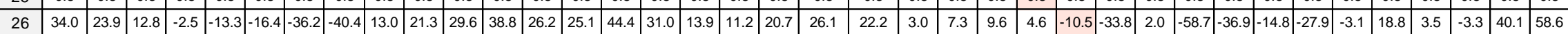

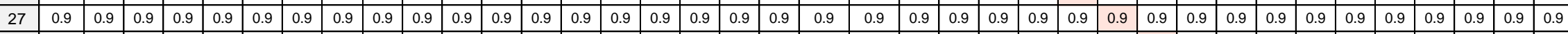
\begin{tabular}{l|l|l|l|l|l|l|l|l|l|l|l|l|l|l|l|l|l|l|l|l|l|l|l|l|l|l|l|l} 
& 0.9 & 0.9 & 0.9 & 0.9 & 0.9 & 0.9 & 0.9 & 0.9 & 0.9 & 0.9 & 0.9 & 0.9 & 0.9 & 0.9 & 0.9 & 0.9 & 0.9 & 0.9 & 0.9 & 0.9 & 0.9 & 0.9 & 0.9 & 0.9 & 0.9 & 0.9 & 0.9 \\
\hline 28 & 0.9 & 0.9 & 0.9 & 0.9 & 0.9 & 0.9 & 0.9 & 0.9 & 0.9 & 0.9 & 0.9 & 0.9 & 0.9 & 0.9 & 0.9 & 0.9 & 0.9 & 0.9 & 0.9 & 0.9 & 0.9 & 0.9 & 0.9 \\
\hline
\end{tabular}

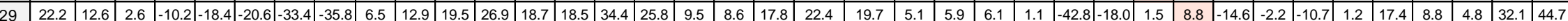
\begin{tabular}{l|l|l|l|l|l|l|l|l|l|l|l|l|l|l|l|l|l|l|l|l|l|l|l|l|l|l|l|l|l|l|l|l|l|l|l|l|l|l|}
\hline 30 & 0.9 & 0.9 & 0.9 & 0.9 & 0.9 & 0.9 & 0.9 & 0.9 & 0.9 & 0.9 & 0.9 & 0.9 & 0.9 & 0.9 & 0.9 & 0.9 & 0.9 & 0.9 & 0.9 & 0.9 & 0.9 & 0.9 & 0.9 & 0.9 & 0.9 & 0.9 & 0.9 & 0.9 & 0.9 & 0.9 & 0.9 & 0.9 & 0.9 & 0.9 & 0.9 & 0.9 & 0.9 & 0.9 \\
\hline
\end{tabular}

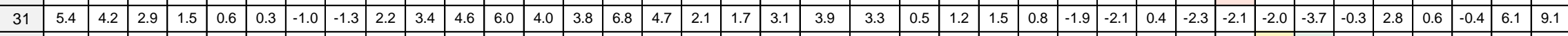

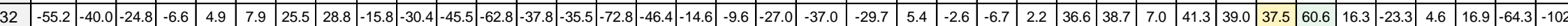

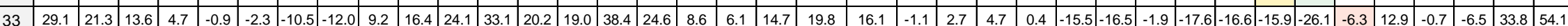

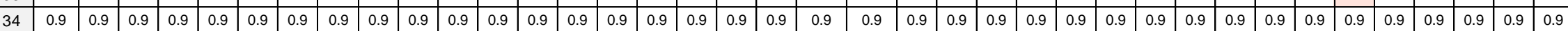

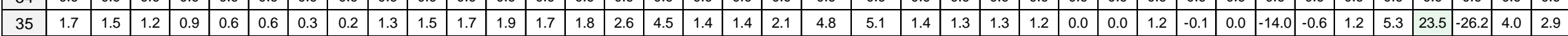

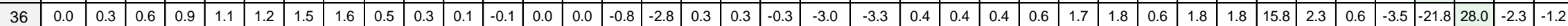

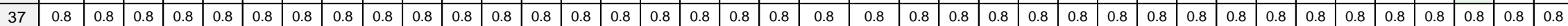

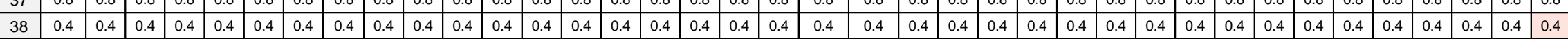


Tabla 4.7. Caudales de fuga obtenidos con 10 sensores de presión (nodos 2, 5, 8, 18, 19, 20, 27, 30, 32, 36)

\begin{tabular}{|l|l|l|l|l|l|l|l|l|l|l|l|l|l|l|l|l|l|l|l|l|l|l|l|l|l|l|l|l|l|l|l|l|l|l|l|l|l|}
\hline 1 & 2 & 3 & 4 & 5 & 6 & 7 & 8 & 9 & 10 & 11 & 12 & 13 & 14 & 15 & 16 & 17 & 18 & 19 & 20 & 21 & 22 & 23 & 24 & 25 & 26 & 27 & 28 & 29 & 30 & 31 & 32 & 33 & 34 & 35 & 36 & 37 & 38 \\
\hline
\end{tabular}

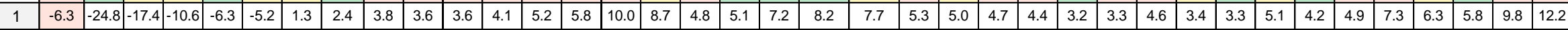

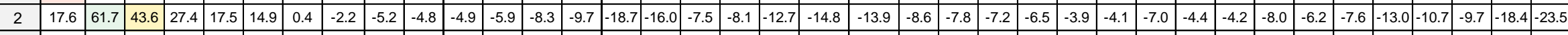
\begin{tabular}{l|l|l|l|l|l|l|l|l|l|l|l|l|l|l|l|l|l|l|l|l|l|l|l|l|l|l|l|l|l|l|l|lllllll}
\hline 3 & -7.7 & -31.7 & -21.6 & -12.9 & -7.6 & -6.3 & 1.2 & 2.5 & 4.1 & 3.8 & 3.9 & 4.4 & 5.6 & 6.3 & 10.8 & 9.5 & 5.2 & 5.5 & 7.8 & 8.8 & 8.4 & 5.7 & 5.3 & 5.1 & 4.7 & 3.4 & 3.5 & 4.9 & 3.6 & 3.5 & 5.5 & 4.6 & 5.3 & 8.0 & 6.8 & 6.3 & 10.6 & 13.1 \\
\hline 4
\end{tabular}

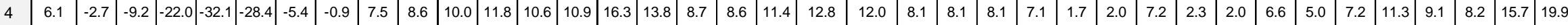

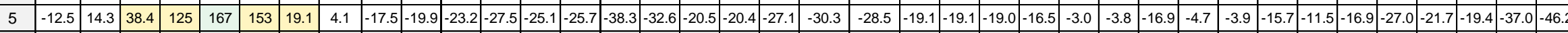

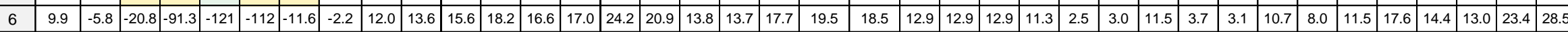

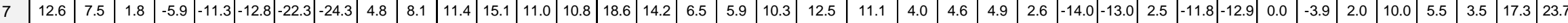

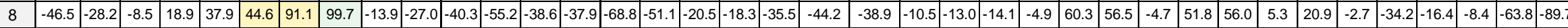

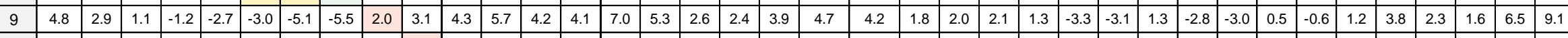

\begin{tabular}{llllllllllllllllllllllllllllllllllllllllllllllllllll}
10 & 0.8 & 0.8 & 0.8 & 0.8 & 0.8 & 0.8 & 0.8 & 0.8 & 0.8 & 0.8 & 0.8 & 0.8 & 0.8 & 0.8 & 0.8 & 0.8 & 0.8 & 0.8 & 0.8 & 0.8 & 0.8 & 0.8 & 0.8 & 0.8 & 0.8 & 0.8 & 0.8 & 0.8 & 0.8 & 0.8 & 0.8 & 0.8 & 0.8 & 0.8 & 0.8 & 0.8 & 0.8 & 0.8 \\
\hline
\end{tabular}

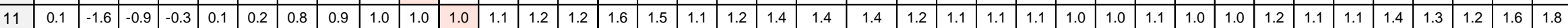

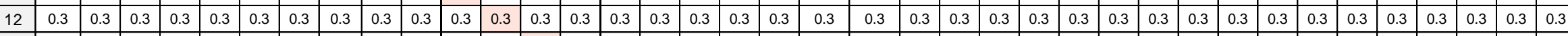

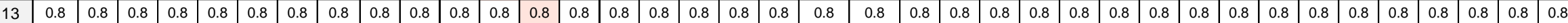

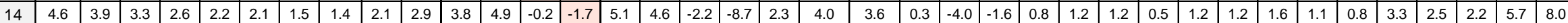

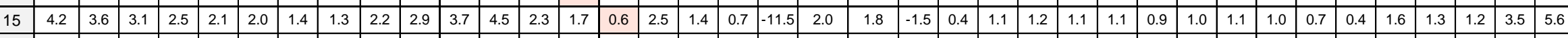

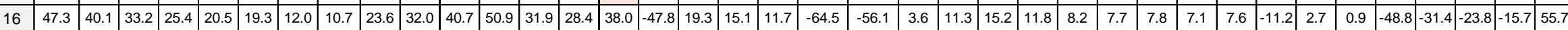
\begin{tabular}{l|l|l|l|l|l|l|l|l|l|l|l|l|l|l|l|l|l|l|l|l|l|l|l|l|l|l|l|l|l|l|lllllllll}
17 & 2.8 & 2.4 & 2.1 & 1.7 & 1.5 & 1.5 & 1.1 & 1.1 & 1.4 & 1.9 & 2.4 & 3.0 & 0.3 & -0.4 & 3.1 & 2.8 & -0.6 & -3.3 & 1.6 & 2.5 & 2.3 & 0.6 & -1.4 & -0.3 & 0.8 & 1.0 & 1.0 & 0.6 & 1.0 & 1.0 & 1.2 & 0.9 & 0.8 & 2.1 & 1.7 & 1.5 & 3.5 & 5.0 \\
\hline
\end{tabular}

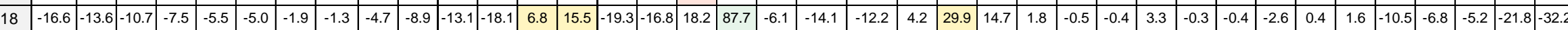

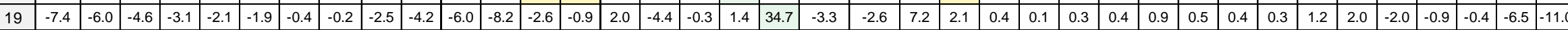

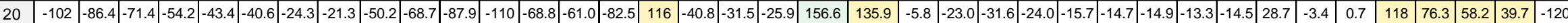

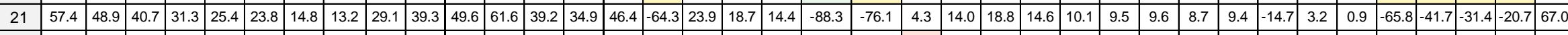

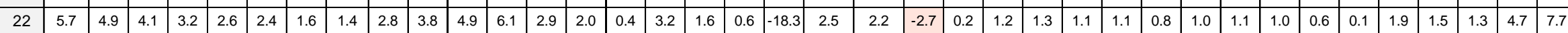

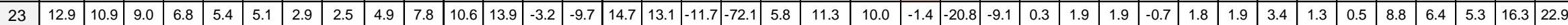

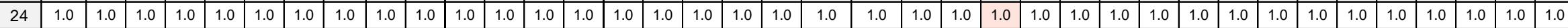

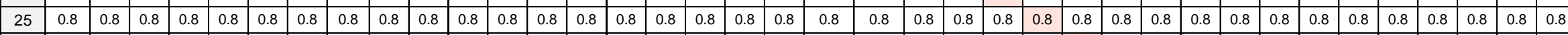

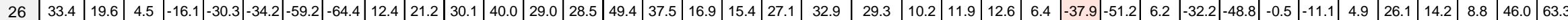

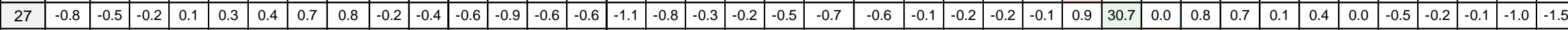

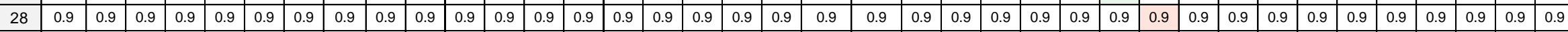

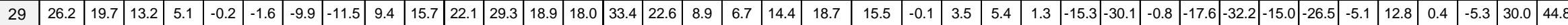

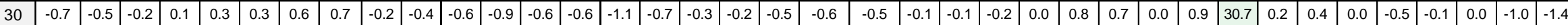

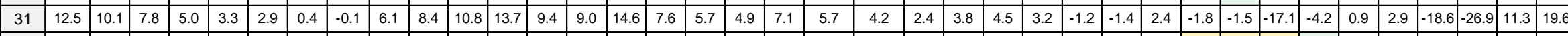

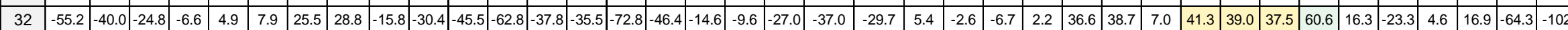

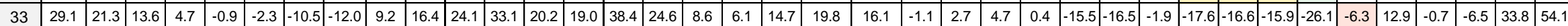

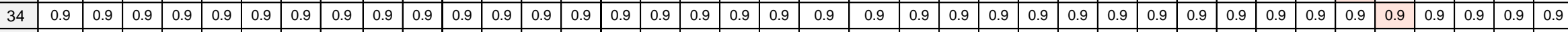

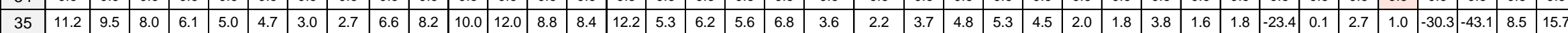

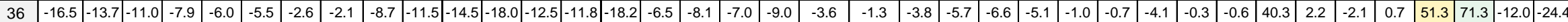

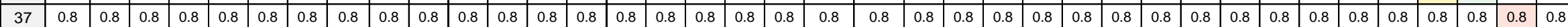

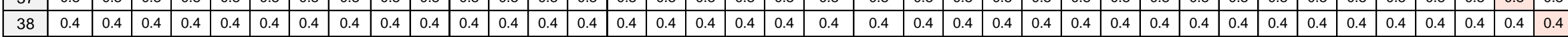


Tabla 4.8. Caudales de fuga obtenidos con 10 sensores de presión y 5 caudalímetros (nudos 2, 5, 8, 18, 19, 20, 27, 30, 32, 36 y tuberías 11, 17, 25, 36, 42)

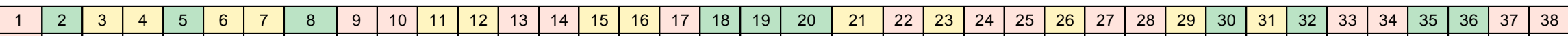

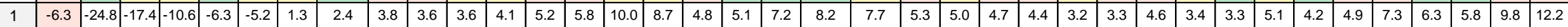

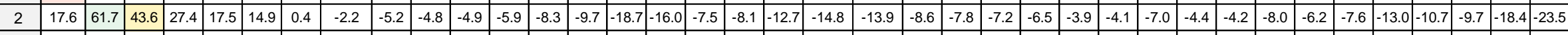

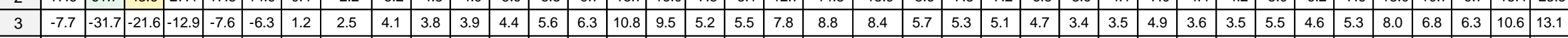

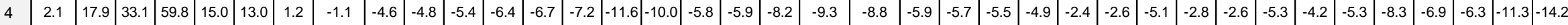

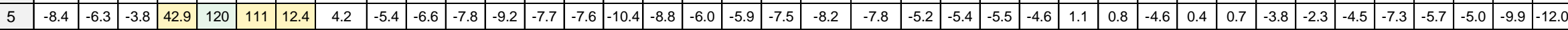

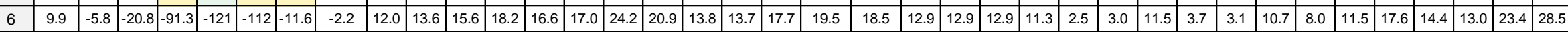

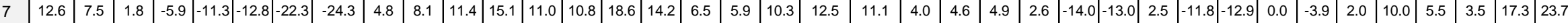

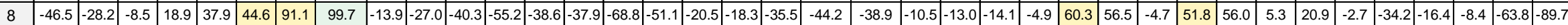

\begin{tabular}{|l|l|l|l|l|l|l|l|l|l|l|l|l|l|l|l|l|l|l|l|l|l|l|l|l|l|l|l|l|l|l|l|llllllll}
\hline 9 & 4.8 & 2.9 & 1.1 & -1.2 & -2.7 & -3.0 & -5.1 & -5.5 & 2.0 & 3.1 & 4.3 & 5.7 & 4.2 & 4.1 & 7.0 & 5.3 & 2.6 & 2.4 & 3.9 & 4.7 & 4.2 & 1.8 & 2.0 & 2.1 & 1.3 & -3.3 & -3.1 & 1.3 & -2.8 & -3.0 & 0.5 & -0.6 & 1.2 & 3.8 & 2.3 & 1.6 & 6.5 & 9.1 \\
\hline
\end{tabular}

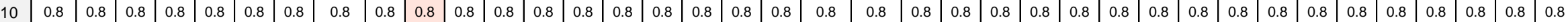

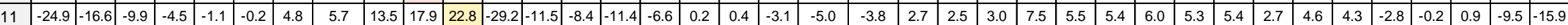
\begin{tabular}{lllllllllllll|l|l|l|l|l|l|l|l|l|l|l|l|l|l|l|l|l|l|l|lllllllllll}
\hline 12 & 25.3 & 15.3 & 9.2 & 4.5 & 1.5 & 0.7 & -3.7 & -4.5 & -12.2 & -16.6 & -21.5 & 30.5 & 12.9 & 9.8 & 13.3 & 8.3 & 1.2 & 1.1 & 4.7 & 6.7 & 5.5 & -1.3 & -1.1 & -1.6 & -6.1 & -4.3 & -4.2 & -4.6 & -4.1 & -4.2 & -1.2 & -3.3 & -2.9 & 4.4 & 1.8 & 0.6 & 11.4 & 18.0 \\
\hline
\end{tabular} \begin{tabular}{llllllllllllllllllllllllllllllllllllllllllllllllllllllll}
\hline 13 & 0.8 & 0.8 & 0.8 & 0.8 & 0.8 & 0.8 & 0.8 & 0.8 & 0.8 & 0.8 & 0.8 & 0.8 & 0.8 & 0.8 & 0.8 & 0.8 & 0.8 & 0.8 & 0.8 & 0.8 & 0.8 & 0.8 & 0.8 & 0.8 & 0.8 & 0.8 & 0.8 & 0.8 & 0.8 & 0.8 & 0.8 & 0.8 & 0.8 & 0.8 & 0.8 & 0.8 & 0.8 & 0.8 \\
\hline
\end{tabular}

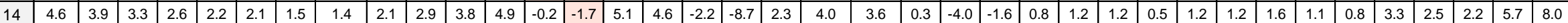
\begin{tabular}{l|l|l|l|l|l|l|l|l|l|l|l|l|l|l|l|l|l|l|l|l|l|l|l|l|l|l|l|l|llllllllll}
15 & 0.2 & 0.2 & 0.1 & 0.1 & 0.1 & 0.1 & 0.0 & 0.0 & 0.1 & 0.2 & 0.2 & 0.2 & 2.4 & 3.9 & 15.6 & 1.5 & 1.0 & 1.1 & -5.9 & 1.2 & 1.0 & -1.0 & 0.4 & 0.6 & 0.1 & 0.0 & 0.0 & 0.0 & 0.0 & 0.0 & 0.2 & 0.0 & -0.1 & 0.8 & 0.5 & 0.4 & 1.9 & 2.9 \\
\hline
\end{tabular}

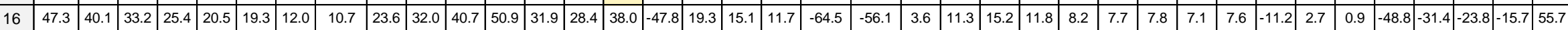

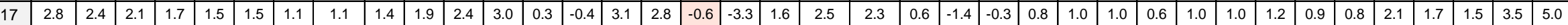

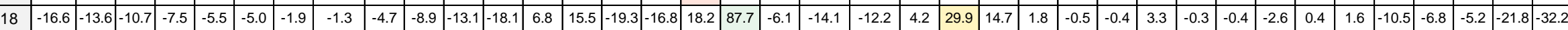

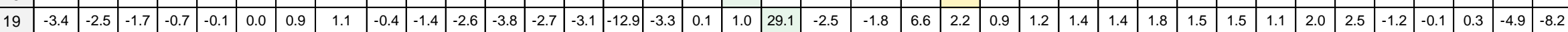

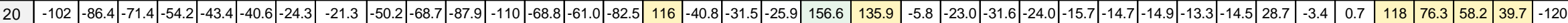

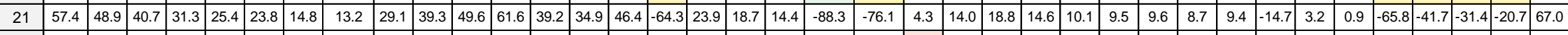

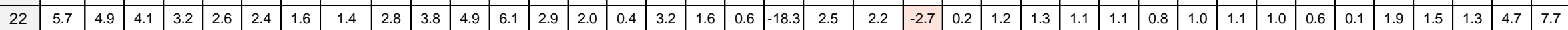

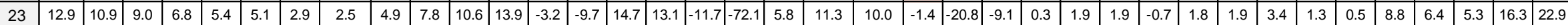

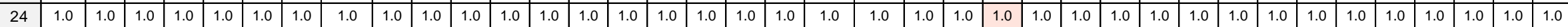
\begin{tabular}{|l|l|l|l|l|l|l|l|l|l|l|l|l|l|l|l|l|l|l|l|l|l|l|l|l|l|l|l|l|l|lllllllllll}
\hline 25 & 0.8 & 0.8 & 0.8 & 0.8 & 0.8 & 0.8 & 0.8 & 0.8 & 0.8 & 0.8 & 0.8 & 0.8 & 0.8 & 0.8 & 0.8 & 0.8 & 0.8 & 0.8 & 0.8 & 0.8 & 0.8 & 0.8 & 0.8 & 0.8 & 0.8 & 0.8 & 0.8 & 0.8 & 0.8 & 0.8 & 0.8 & 0.8 & 0.8 & 0.8 & 0.8 & 0.8 & 0.8 & 0.8 \\
\hline
\end{tabular}

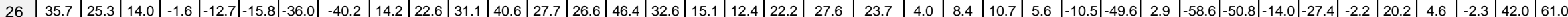

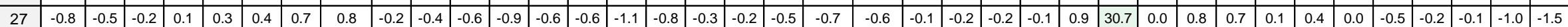

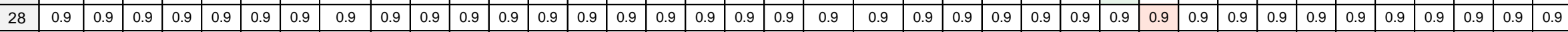

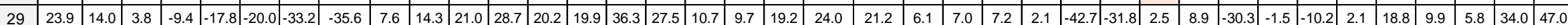

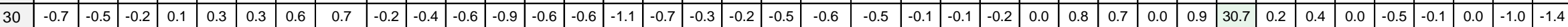

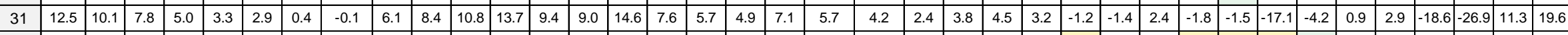

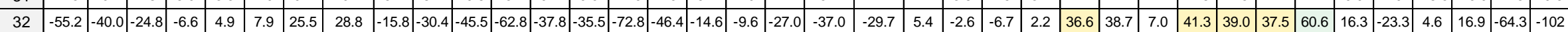

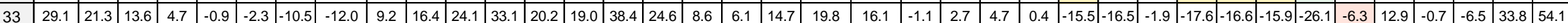

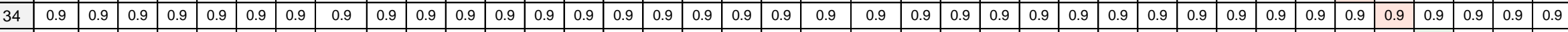

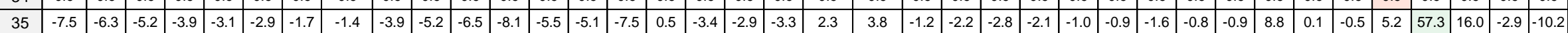

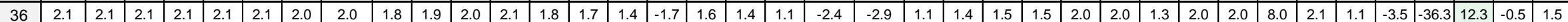
\begin{tabular}{|l|l|l|l|l|l|l|l|l|l|l|l|l|l|l|l|l|l|l|l|l|l|l|l|l|l|l|l|l|l|l|lllllllll}
\hline 37 & 0.8 & 0.8 & 0.8 & 0.8 & 0.8 & 0.8 & 0.8 & 0.8 & 0.8 & 0.8 & 0.8 & 0.8 & 0.8 & 0.8 & 0.8 & 0.8 & 0.8 & 0.8 & 0.8 & 0.8 & 0.8 & 0.8 & 0.8 & 0.8 & 0.8 & 0.8 & 0.8 & 0.8 & 0.8 & 0.8 & 0.8 & 0.8 & 0.8 & 0.8 & 0.8 & 0.8 & 0.8 & 0.8 \\
\hline
\end{tabular}

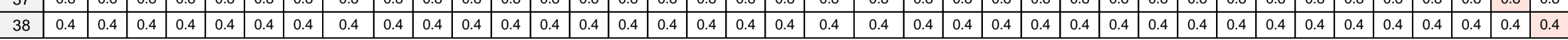


Tabla 4.9. Caudales de fuga obtenidos con 15 sensores de presión (nudos 2, 5, 8, 10, 13, 18, 19, 20, 24, 27, 28, 30, 32, 34, 36)

\begin{tabular}{|c|c|c|c|c|c|c|c|c|c|c|c|c|c|c|c|c|c|c|c|c|c|c|c|c|c|c|c|c|c|c|c|c|c|c|c|c|c|}
\hline 1 & 2 & 3 & 4 & 5 & 6 & 7 & 8 & 9 & 10 & 11 & 12 & 13 & 14 & 15 & 16 & 17 & 18 & 19 & 20 & 21 & 22 & 23 & 24 & 25 & 26 & 27 & 28 & 29 & 30 & 31 & 32 & 33 & 34 & 35 & 36 & 37 & 38 \\
\hline
\end{tabular}

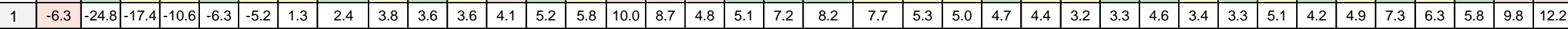

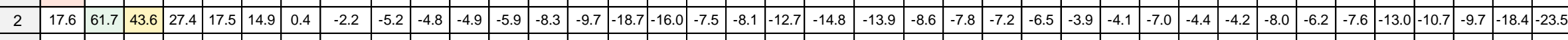

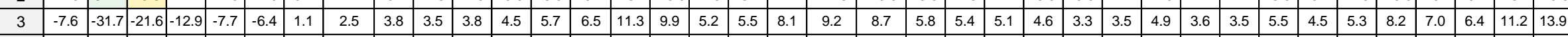

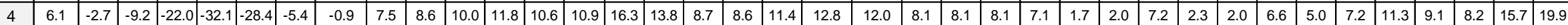

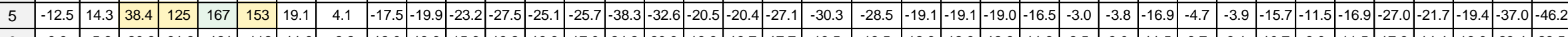

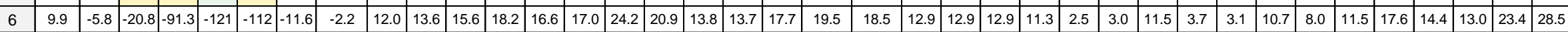

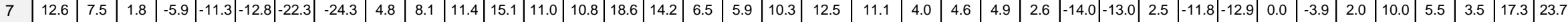

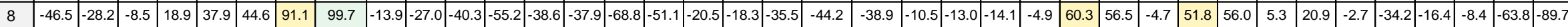

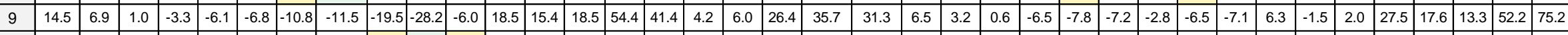

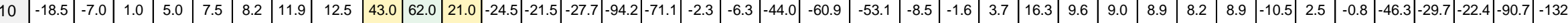

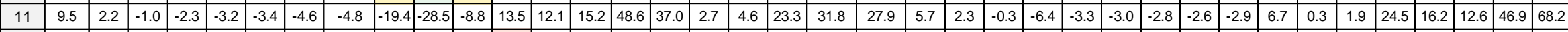

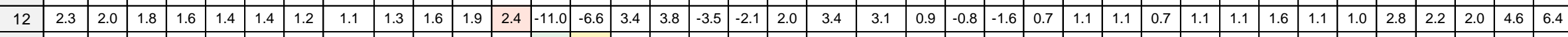

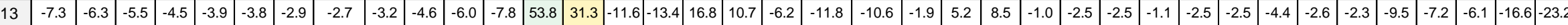

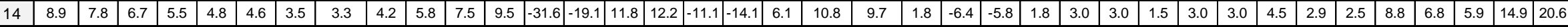

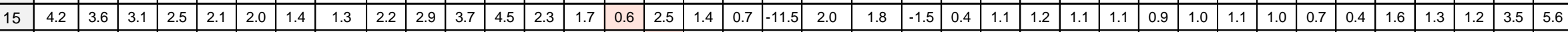

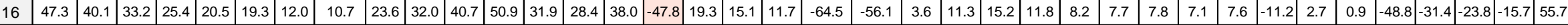

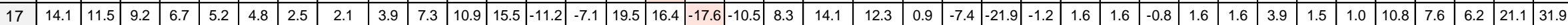

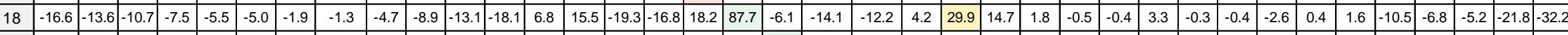

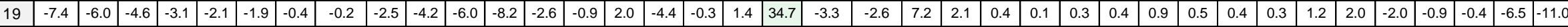

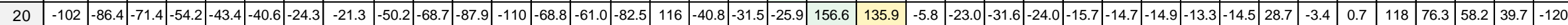

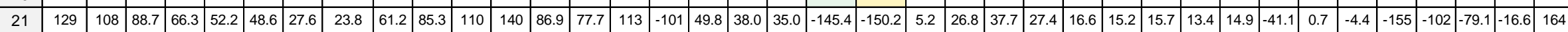

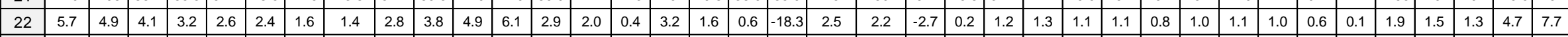

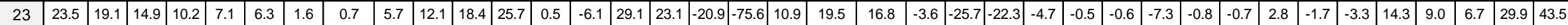

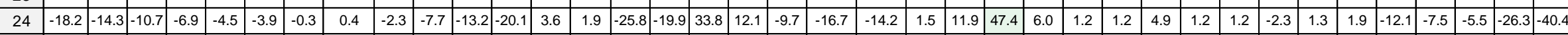

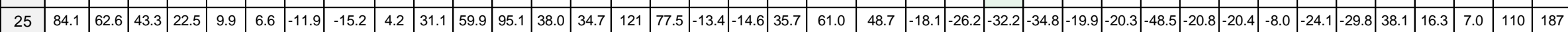

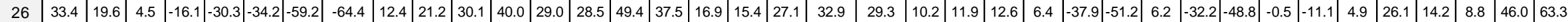

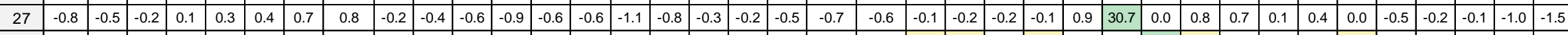

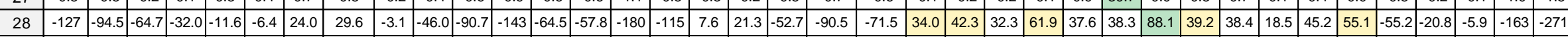

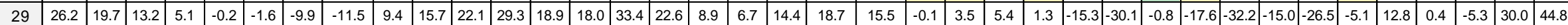

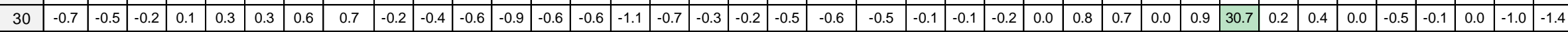

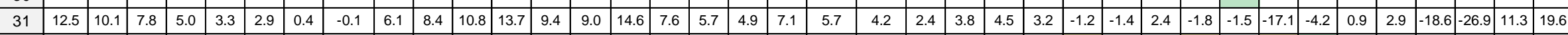

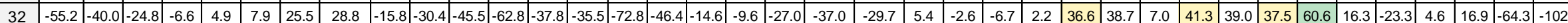

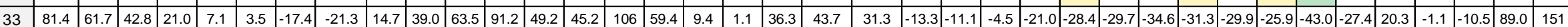

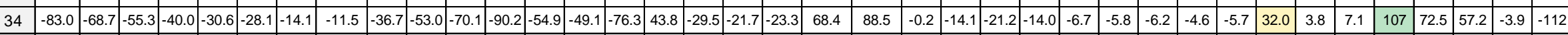

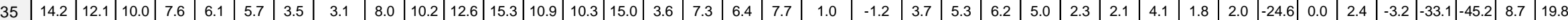

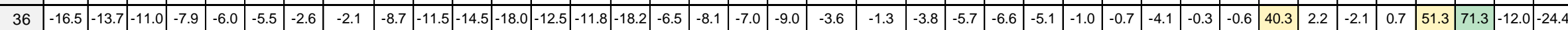

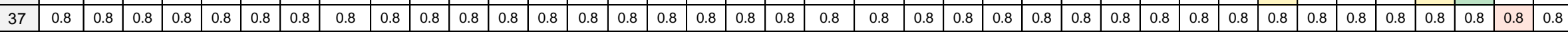

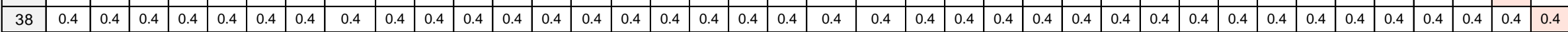




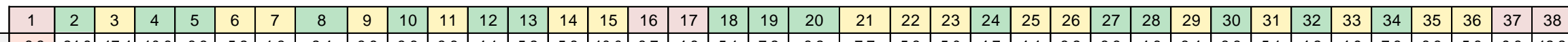

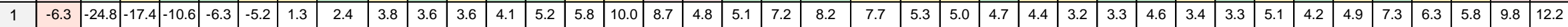

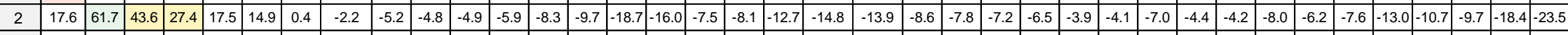

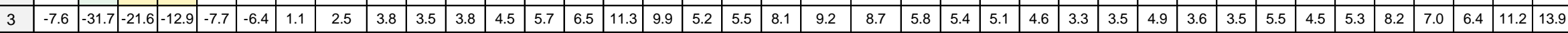

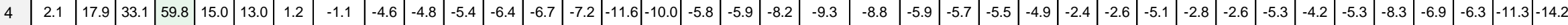

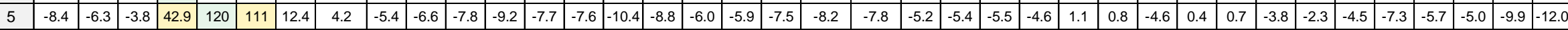

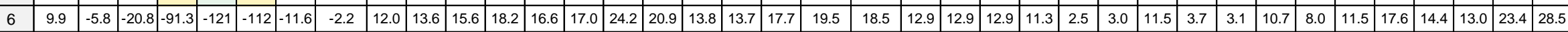

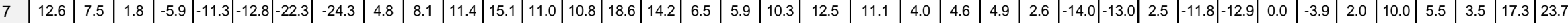

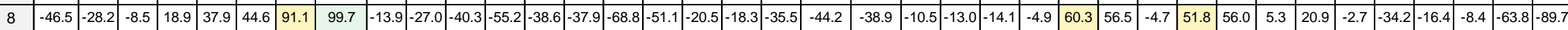

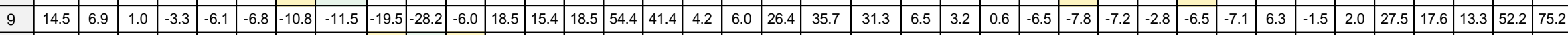

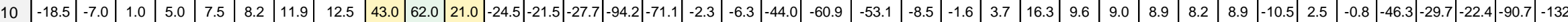

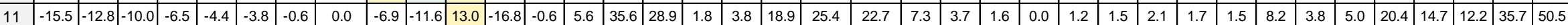

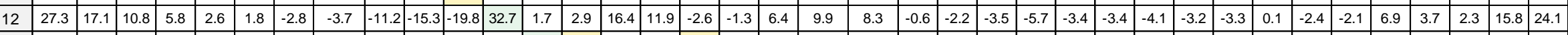

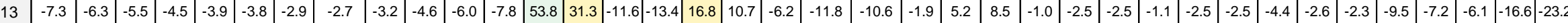

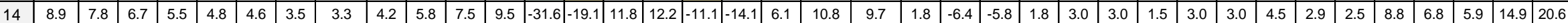

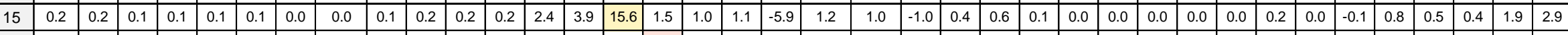

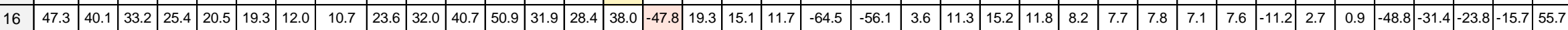

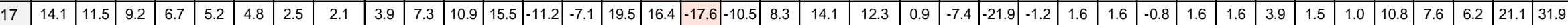

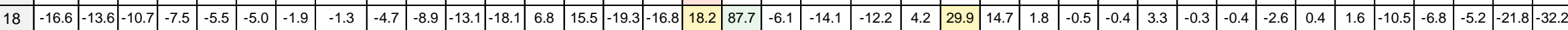

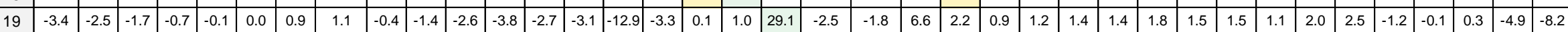

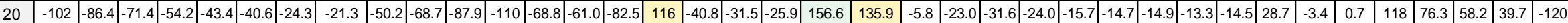

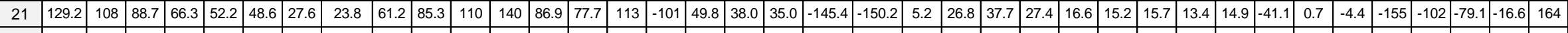

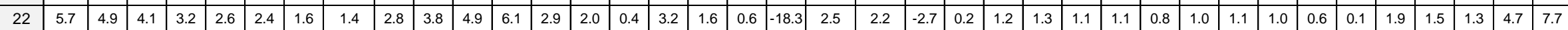

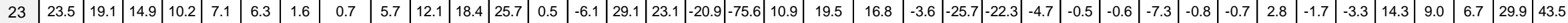

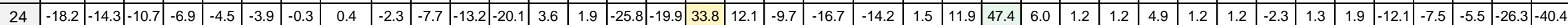

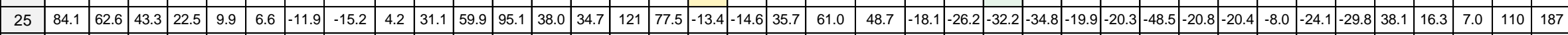

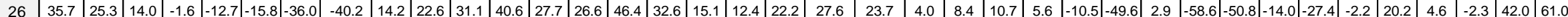

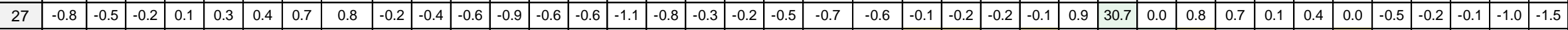

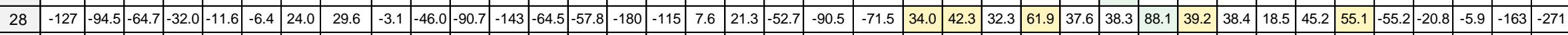

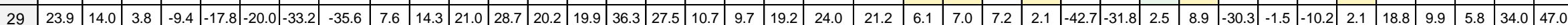

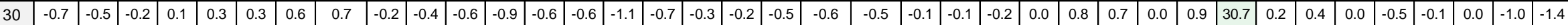

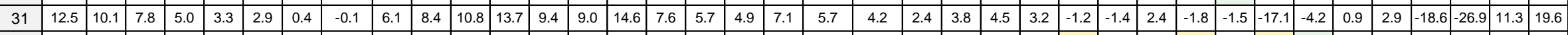

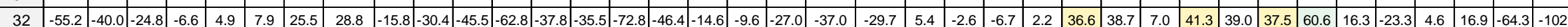

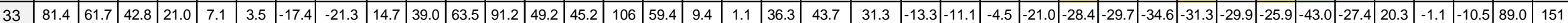

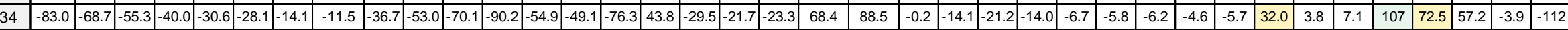

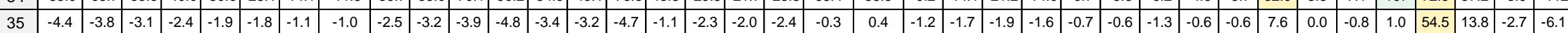

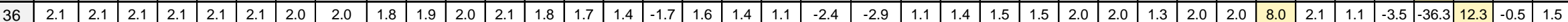

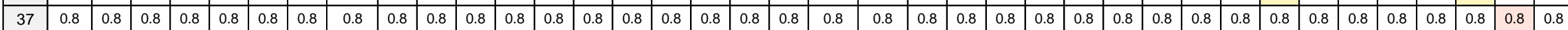

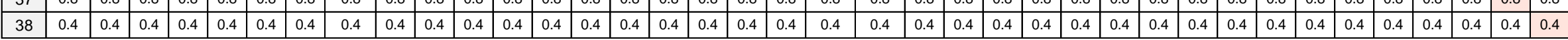




\section{Capítulo 5 \\ Casos de estudio}

\subsection{Introducción}

Este trabajo tiene como objetivo fundamental plantear técnicas de detección y localización de los consumos incontrolados, principalmente los fugados no declarados, y que además presenten un valor añadido a la gestión integral del sistema. Para ello, en los capítulos 2 y 3 se ha estudiado la técnica de la sectorización y propuesto una metodología para realizar el diseño de esta. Así, se ha conseguido organizar toda la red en sectores bajo criterios energéticos minimizando el aporte subjetivo del diseñador. El capítulo 4 aborda el uso de sensores para la ubicación de consumos incontrolados, permitiendo identificar la zona de ocurrencia.

Ambas técnicas presentan contundentes ventajas y ligeros inconvenientes para la gestión global de las redes de distribución de agua. La sectorización de las redes se ha definido como una estrategia adecuada porque reduce el área de inspección y, en consecuencia, reduce la duración de las fugas. En contrapartida, supone una ramificación de las redes $^{2}$ y una disminución de la fiabilidad del suministro. En el caso de la sensorización de las redes, los resultados pueden arrojar ubicaciones precisas en menor tiempo sin reducir la capacidad hidráulica de las infraestructuras al ser una técnica no intrusiva. No obstante, la gestión de una gran cantidad de datos supone mayores costes, sin los beneficios de la sectorización (reducción del área de búsqueda de anomalías).

\footnotetext{
${ }^{2}$ Una red de agua a presión se denomina ramificada si se cumple que el número de mallas $\mathrm{M}=0$. El número de mallas cumple la relación $\mathrm{M}=\mathrm{L}-\mathrm{N}+1$ (siendo $\mathrm{N}$ el número de nudos y $\mathrm{L}$ el número de tuberías). En este contexto "la red se ramifica" significa que disminuye el número de trayectos por los que realizar el abastecimiento, es decir, que se cierran tuberías en el proceso de sectorización.
} 
A priori, analizar cuán buena es cada una de las metodologías abordadas no es tarea fácil debido a la cantidad de variables que entran en juego. Para ello, en este capítulo se analiza detalladamente el grado de cumplimiento de unos objetivos claros, precisos, medibles y alcanzables. Estos se cuantifican con unos indicadores de gestión y según unos criterios de evaluación que permitan valorar los logros y deficiencias de cada técnica.

El sistema de evaluación debe disponer de una misma escala de valores para todas las métricas, de modo que se evalúe cada métrica por separado y permita su comparación. Se pretende cuantificar los volúmenes incontrolados y su efecto global sobre los sistemas de distribución de agua.

Una vez establecido el método de evaluación, todo ello se aplica sobre dos casos de estudio. El primero de ellos consiste en la red sintética descrita en los capítulos 3 y 4 , un sistema sencillo desde el punto de vista hidráulico, que describe cada proceso y facilita la comprensión de los resultados obtenidos. En el segundo caso, se ha seleccionado un sistema urbano de distribución de agua potable real, cuyo objetivo será analizar el alcance de las metodologías y estudiar las limitaciones reales impuestas por las infraestructuras actuales.

\subsection{Sistema de evaluación}

Como se ha visto hasta ahora, los sistemas de distribución de agua quedan definidos por multitud de variables. Estas, aun estando relacionadas entre sí, se miden en diferentes unidades y los objetivos marcados definen la importancia (el peso) de cada una en un proceso de toma de decisión. Cuando se evalúa la idoneidad de un proceso, se debe definir correctamente qué (bajo qué criterios) y cómo (siguiendo qué indicadores) se realiza la evaluación.

Se cuantifica el alcance de las metodologías frente a la gestión de los volúmenes incontrolados, y se cuantifican los efectos negativos que su aplicación pudiera ocasionar al sistema. Es necesario establecer un procedimiento que evalúe los objetivos de manera global y específica. El sistema de evaluación se organiza en tres niveles: objetivos, criterios y métricas de evaluación (Figura 5.1).

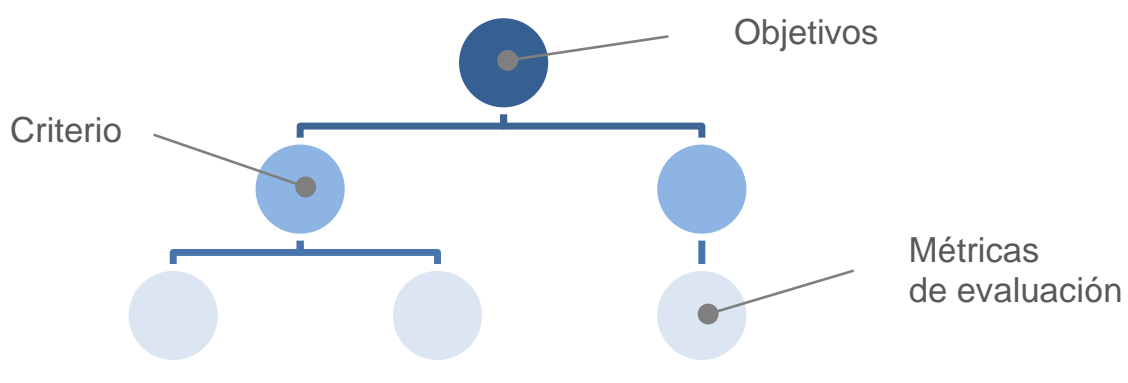

Figura 5.1 Estructura del sistema de evaluación

Los objetivos generales se plantean desde una perspectiva global y definen el grado de satisfacción logrado. Cabe destacar que los objetivos deben ser reales, precisos, medibles y alcanzables en el tiempo. Asimismo, no todos los objetivos tienen porqué tener el mismo grado de repercusión o la misma importancia para el gestor. 
El siguiente paso consiste en la selección de unos criterios de evaluación que informen sobre el grado de consecución de tales objetivos. Se trata de profundizar un nivel en la visión del análisis y focalizar, dentro del objetivo, el ámbito más concreto a evaluar. Los criterios de evaluación se encargan de cuantificar los objetivos establecidos. Al igual que en el caso anterior, el gestor puede establecer diferentes grados de importancia para cada uno de los criterios de evaluación de cada objetivo.

En último lugar, y llegados al nivel más bajo de detalle, se establecen las métricas de evaluación de los criterios establecidos. Las métricas no son más que indicadores de gestión (Cabrera Jr. \& Pardo, 2008), los cuales servirán para medir los procesos del trabajo. Numerosos indicadores se han definido para mostrar la gestión en redes de distribución de agua potable (Cabrera, 2018). Muchos de ellos se han establecido de manera absoluta (por ejemplo, número de fugas por año o volumen consumido anual), por lo que el gestor no puede comparar sus resultados con otras redes. Para ello, y así se establece en el presente trabajo, se deben seleccionar métricas que sí admitan comparación, (número de fugas por kilómetro de red y año, volumen consumido por abonado y año, etc.).

Cada métrica debe tener su propia importancia o peso, al igual que sucede con los objetivos con respecto a los criterios.

Para asociar el peso en los objetivos, criterios y métricas, se han adoptado cinco niveles de importancia (Coelho, 1997) y, para cada nivel, un peso asociado (0,50 - muy bajo, 0,75 - bajo, 1,00 - medio, 1,50 - alto, 2,00 - muy alto). El gestor de la red tiene el objetivo de asociar el peso correspondiente en función de su experiencia o de los criterios a seguir. Y los pesos asignados se fijan de manera independiente del resto, no siendo necesario que cada subgrupo de objetivos, criterios o métricas alcancen un peso total determinado.

\subsubsection{Método de cálculo adoptado}

Una vez establecida la estructura de evaluación de las metodologías objeto de este trabajo y el peso de cada elemento evaluador, el siguiente paso consiste en establecer el cálculo cuantitativo de los objetivos, criterios y métricas. Para ello, y en orden inverso al seguido en su definición, se comienza la evaluación por los niveles inferiores, las métricas, para posteriormente evaluar los criterios, los objetivos y, finalmente, la evaluación global de la metodología objeto de estudio.

Para la evaluación de las métricas, es importante destacar que cada una de ellas puede tener unas unidades diferentes, en función de su naturaleza. El valor de cada métrica asume una importancia diferente según las decisiones impuestas por el gestor y muchas veces se ha de cambiar unidades. Para ello, se definen tres rangos cualitativos: bueno, regular y malo, así como una relación lineal entre los valores cualitativos y el rango cuantitativo de evaluación (de 0 a 10). Por ejemplo, para el número de roturas por kilómetro de red y año (Figura 5.2) se han fijado tres rangos cualitativos. El primero (cualitativamente bueno) comprende todos los valores entre 0 y 1 rotura $/ \mathrm{km} / a n ̃ o$. Los valores entre 1 y 2,5 roturas $/ \mathrm{km} / \mathrm{año}$, se etiquetan como valores regulares. Y son malos los valores mayores de 2,5 roturas $/ \mathrm{km} / \mathrm{año}$. Esta clasificación, cualitativa, se puede complementar con una clasificación cuantitativa estableciendo, para ello, una relación lineal entre los rangos establecidos y una valoración de 0 a 10. Así, el rango de valores buenos tendrá una valoración entre 6,67 y 10, la valoración de los valores regulares estará entre 3,33 y 6,67 y a los valores malos, aquellos menores de 2,5 fugas/km/año, se les asignará una valoración entre 0 y 3,33. Siguiendo todo lo establecido, un valor de 0,7 
fugas/km/año en la nueva escala quedará etiquetado como un valor bueno y le corresponde una valoración de 7,67, en una escala de 0 a 10. En cambio, un valor de 5,1 fugas/km/año queda como un valor malo cuya valoración corresponde con un 0,86 . Este cambio en las unidades permitirá normalizar todas las métricas necesarias para un intervalo específico (entre 0 y 10), independientemente de sus naturalezas.

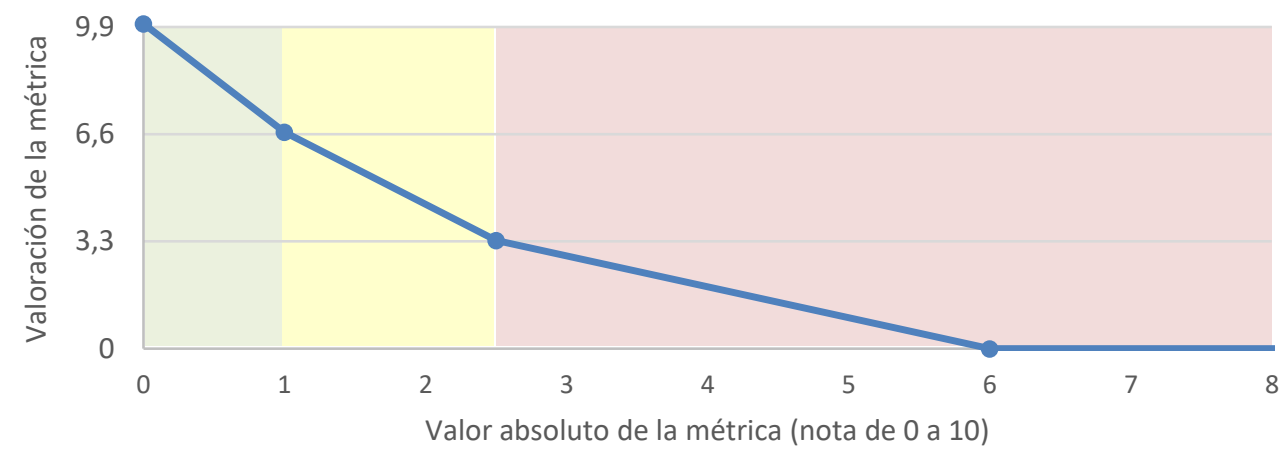

Figura 5.2 Evaluación de las métricas según rangos definidos (bueno, regular y malo; verde, amarillo y rojo)

Posteriormente se evalúan los criterios. Para ello, se emplea la ecuación 5.1 que contempla los valores numéricos de las métricas y sus pesos.

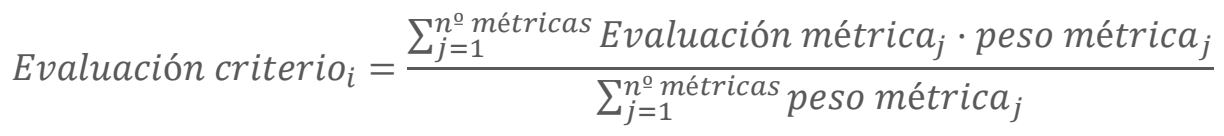

A partir de las valoraciones de todos los criterios, se puede obtener la evaluación de cada objetivo (ecuación 5.2).

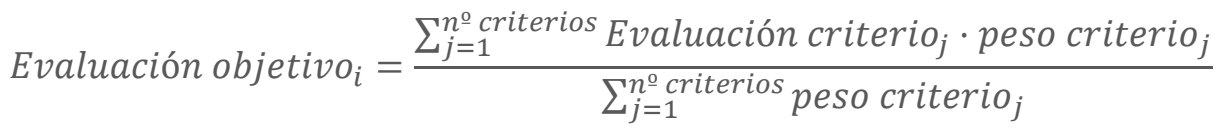

Como último paso, se obtiene la valoración global de la metodología (ecuación 5.3) que considera la evaluación global con las evaluaciones de todos los objetivos y sus pesos.

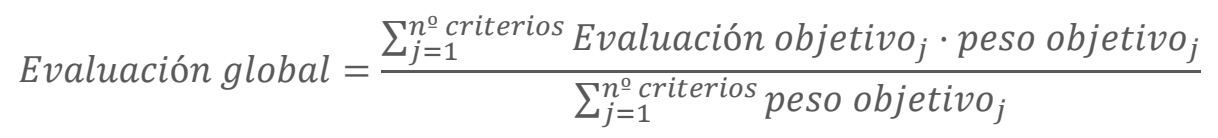

\subsubsection{Objetivos de evaluación seleccionados}

Se propone evaluar cómo afectan las metodologías desarrolladas (sectorización y sensorización) sobre cuatro grandes líneas de la gestión de los sistemas de distribución de agua. En primer lugar, se quiere conocer cómo afectará su aplicación sobre los trabajos de búsqueda de fugas. Acto seguido, es necesario evaluar si la implantación de las metodologías afectará, de algún modo, a la capacidad hidráulica de la red y a la calidad del agua. En último lugar, se evalúa el coste de implantación de cada metodología. Los cuatro objetivos se describen a continuación:

- Objetivo 1 - Efectividad y eficiencia en la búsqueda de fugas no declaradas: Este primer objetivo ( $\mathrm{y}$ tal vez el más importante de todos debido a que recoge el espíritu 
del trabajo), evalúa la precisión y la rapidez con la que se detectan y ubican las fugas no declaradas. Es decir, se evaluará identifica qué si la aplicación de las metodologías y en qué cantidad optimiza hace más eficientes y eficaces los trabajos de búsqueda de fugas.

- Objetivo 2 - Capacidad hidráulica de la red resultante: El mero hecho de sectorizar, al ser una técnica intrusiva, modificará la definición inicial de las redes. Con este segundo objetivo se pretende evaluar cómo se verá modificada esta capacidad ante la implementación de las técnicas.

- Objetivo 3 - Calidad del agua de la red resultante: Si cualquier técnica modifica los caminos que tiene que recorrer el fluido para satisfacer la demanda, se alteran los tiempos de permanencia del agua en red y, con ello, todas las variables que afectan a la calidad del agua. Por tanto, se identifican las posibles variaciones de dichas variables.

- Objetivo 4 - Coste de implantación: El último objetivo establecido cuantifica directa o indirectamente el coste necesario para su implementación.

Respecto a la importancia de cada uno de ellos (Tabla 5.1), se ha establecido una importancia máxima a la efectividad y eficiencia en la búsqueda de fugas no declaradas, un grado menor a la afección sobre la capacidad hidráulica del sistema y la calidad del agua, y finalmente se le he otorgado menos relevancia al coste de implantación. Cabe destacar que este reparto de pesos depende de la importancia que adquiera cada uno de los objetivos para el gestor que implemente la técnica en cuestión.

Tabla 5.1 Pesos asociados a cada objetivo

\begin{tabular}{|l|c|}
\hline & Peso \\
\hline Objetivo 1 - Efectividad y eficiencia en la búsqueda de fugas no declaradas & 2 \\
\hline Objetivo 2 - Capacidad hidráulica de la red resultante & 1,5 \\
\hline Objetivo 3 - Calidad del agua de la red resultante & 1,5 \\
\hline Objetivo 4 - Coste de Implantación & 1 \\
\hline
\end{tabular}

\subsubsection{Criterios de evaluación seleccionados}

Para conocer cómo las metodologías propuestas influyen en los trabajos de búsquedas de fugas se han propuesto 9 criterios de evaluación (Tabla 5.2). Para evaluar cómo afectan las metodologías desarrolladas sobre los trabajos de búsquedas de fugas, se han seleccionado dos criterios. En primer lugar, se ha establecido el coste anual de dichos trabajos y, en segundo lugar, el tiempo empleado para localizar las fugas una vez detectadas. En ambos casos se busca una reducción de estos. Desde la óptica hidráulica, se han seleccionado la presión hidráulica de la red (indicador directo del estado la red), la resiliencia (estado de la red ante cambios) y la energía disponible en la red para analizar posibles modificaciones en su reparto. La calidad del agua se evalúa con el tiempo de permanencia en la red y con los niveles del cloro en las tuberías. En último lugar, se analizan los costes directos e indirectos necesarios en la implantación de las metodologías.

La Tabla 5.2 recoge los pesos asignados. Cabe destacar que estos valores numéricos se han asignado a cada criterio independientemente del resto. Además, la suma del peso total de los 
criterios bajo cada objetivo no tiene porqué ser la misma, y un mayor peso total no tiene porqué indicar una mayor importancia de dicho objetivo, simplemente indica que hay mayor número de criterios o que la importancia de estos es mayor.

Tabla 5.2 Criterios de evaluación y pesos asociados

\begin{tabular}{|l|c|}
\hline & Peso \\
\hline Criterio 1.1: Coste anual campañas búsqueda & 2 \\
\hline Criterio 1.2: Tiempo de ubicación & 1,5 \\
\hline Criterio 2.1: Presión hidráulica en red & 2 \\
\hline Criterio 2.2: Resiliencia de la red & 1,5 \\
\hline Criterio 2.3: Energía disponible en red & 1 \\
\hline Criterio 3.1: Tiempo de permanencia & 1 \\
\hline Criterio 3.2: Concentración de cloro & 2 \\
\hline Criterio 4.1: Costes directos de implantación & 2 \\
\hline Criterio 4.2: Otros costes & 1 \\
\hline
\end{tabular}

\subsubsection{Métricas de evaluación seleccionadas}

Se han seleccionado 17 métricas que se muestran la Tabla 5.3 organizadas bajo sus objetivos y criterios de evaluación. Se entiende, así, que el diseñador o gestor ha elegido las métricas y su número no está limitado. Por ejemplo, el coste anual de las campañas de búsqueda de fugas (1.1) se determina como la reducción en los costes de los trabajos de búsqueda de fugas más los costes del volumen fugado, y el criterio de presión hidráulica (2.1) se define por cuatro métricas distintas (Tabla 5.3).

Para los casos de estudio recogidos en este capítulo, la selección de las métricas se ha guiado por los siguientes razonamientos.

\section{Objetivo 1}

- 1.1. Coste anual de las campañas de búsqueda. Se evalúa como la variación de los costes anuales debidos al volumen incontrolado fugado y a las campañas de búsqueda de fugas.

- 1.2. Tiempo de ubicación. Se mide según la velocidad de inspección de la red.

Con estas dos métricas se pretender evaluar cuán rápidamente y por cuánto se realizarán los trabajos de búsqueda de fugas bajo los nuevos escenarios planteados por las metodologías.

\section{Objetivo 2}

- 2.1. Presión hidráulica de la red. Se han seleccionado como métricas la variabilidad, el exceso y déficit de presión y una última métrica que evalúa la importancia de la demanda media según la demanda y el nivel de importancia de la presión de cada nudo.

- 2.2. Resiliencia de la red. Se evalúa la robustez de la red con los índices de fiabilidad, de Todini (2000), el índice de robustez (Prasad \& Park, 2004) y con el comportamiento de la red frente a incendio. 
- 2.3. Energía disponible en red. Se calcula la energía entregada a los usuarios y la eficiencia energética dinámica (apartado 3.3.1).

\section{Objetivo 3}

- 3.1, Tiempo de permanencia del agua. Es un parámetro medible y fácil de calcular con un modelo de simulación hidráulica. Se han seleccionado los tiempos máximo y medio de permanencia del agua en las tuberías.

- 3.2. Concentración del cloro en la red. Se emplea para evaluar la concentración mínima y media del cloro en el agua.

\section{Objetivo 4.}

- 4.1. Costes directos de implantación. Se ha seleccionado como métrica de evaluación los costes directos necesarios para la implantación divididos entre el total de la longitud afectada.

- 4.2. Otros costes. En este caso, se han seleccionado los otros costes necesarios.

Se ha asignado un peso máximo (2 de 2) a la variación del coste anual (1.1), la velocidad de inspección de la red (1.2), exceso y déficit en la presión (2.1), índices de fiabilidad y robustez (2.2), a la eficiencia energética dinámica (2.3), al tiempo máximo de permanencia (3.1) y a los costes directos de implantación (4.1). Al resto de métricas se les ha asignado un peso medio (1) o medio-alto $(1,5)$.

Por último, se definen los rangos de valoración, así como sus valores máximos y mínimos (Tabla 5.4). La implantación de las metodologías pretende una reducción en los costes de la búsqueda de fugas y también del tiempo de búsqueda. Se considera un valor bueno si se produce una reducción entre el 50 y el 100\%, regular entre el 10 y el $50 \%$ y malo por debajo del $10 \%$. Si la velocidad de inspección no mejora, se etiqueta como resultado malo, regular si se consiguen entre 300 y 1.000 (m/día de red inspeccionada) y buenos para valores mayores a $1.000 \mathrm{~m} / \mathrm{día}$.

Respecto a la presión hidráulica de la red, interesa que la implantación de las metodologías consiga unos niveles homogéneos de presión, con niveles cercanos a los niveles mínimos de servicio (siempre por encima) y con mayores presiones en aquellos nudos con mayor demanda, para así ofrecer mejor calidad de servicio. Por ello, se ha establecido como rango bueno, las variaciones de presión entre 0 y 20 mca, regular, los valores entre 20 y 40 mca, y malo, los valores mayores de $40 \mathrm{mca}$. El exceso de la presión supone un valor bueno si es menor que 10 mca y regular si es inferior a los 20 mca. En cuanto al déficit de presión, se busca un valor de 0 , ya que valores negativos implican la existencia de nudos con presiones por debajo de la presión mínima de servicio. Por ello, se estable un rango bueno para los valores entre 0 y -2 mca, regulares para aquellos entre -2 y -6 mca, y malos para valores menores.

En el presente trabajo se premia (desde la óptica de la resiliencia) el mejor comportamiento frente a los cambios. Por tanto, el escenario ideal consiste en que todos los nudos sean capaces de mantener su presión en condiciones de incendio y adopten valores iguales a la unidad para los índices de fiabilidad y robustez. En la realidad, se establece que, ante situación de incendio, se considera bueno si menos del 5\% de los nudos presenta una presión menor que la requerida, regular si son entre el 5 y el $10 \%$ de los nudos y como malo si son 
más del $10 \%$. Los índices de fiabilidad y robustez consideran un reparto lineal decreciente entre 1 y 0 , cortando los valores buenos en 0,67 y los regulares en 0,33.

Si se analiza la energía, se considera un escenario bueno aquel en que la energía entregada al usuario excede entre un 0 y $70 \%$ con respecto a la mínima necesaria, regular entre un 70 y $150 \%$ y malo para valores superiores a este último umbral. La eficiencia energética dinámica se considera buena entre 100 y 60, regular entre 40 y 60 y mala para valores inferiores.

El tiempo de permanencia se considera bueno si se incluye entre las 0 y 8 horas, regular entre 8 y 16 horas, y malo para tiempos superiores a las 16 horas. La concentración de cloro se considera buena entre 1 y $0.7 \mathrm{ppm}$, regular entre 0,4 y $0,7 \mathrm{ppm}$ y mala para valores por debajo de 0,4 ppm.

Los rangos establecidos para las dos últimas métricas del objetivo 4, referentes a costes de implantación, dependen de los límites presupuestarios establecidos en cada servicio. 
Tabla 5.3 Métricas de evaluación y pesos asociados

\begin{tabular}{|c|c|c|c|c|c|}
\hline Objetivos & $\begin{array}{l}\text { Criterios de } \\
\text { evaluación }\end{array}$ & Métricas & Definición & Unidad & Peso \\
\hline \multirow{2}{*}{$\begin{array}{l}\text { Objetivo } 1 \\
\text { Efectividad y eficiencia } \\
\text { en la búsqueda de } \\
\text { fugas no declaradas }\end{array}$} & $\begin{array}{c}\text { Criterio } 1.1 \\
\text { Coste anual } \\
\text { campañas búsqueda }\end{array}$ & $\begin{array}{l}\text { Variación en el coste } \\
\text { anual }\end{array}$ & $\begin{array}{l}\text { Variación en los costes anuales debido al volumen de agua fugada y } \\
\qquad \text { a las campañas de búsqueda (Hunaidi, 2005) }\end{array}$ & $\%$ & 2 \\
\hline & $\begin{array}{l}\text { Criterio } 1.2 \\
\text { Tiempo de ubicación }\end{array}$ & $\begin{array}{l}\text { Velocidad de } \\
\text { inspección }\end{array}$ & $\begin{array}{l}\text { Longitud de red inspeccionada por los equipos de búsqueda de fugas } \\
\qquad \text { por unidad de tiempo }\end{array}$ & $\mathrm{m} / \mathrm{h}$ & 2 \\
\hline \multirow{5}{*}{$\begin{array}{l}\text { Objetivo } 2 \\
\text { Capacidad hidráulica de } \\
\text { la red resultante }\end{array}$} & \multirow{4}{*}{$\begin{array}{c}\text { Criterio } 2.1 \\
\text { Presión hidráulica }\end{array}$} & $\begin{array}{l}\text { Variabilidad de la } \\
\text { presión }\end{array}$ & $\begin{array}{l}\text { Desviación media de la presión respecto a la presión de diseño } \\
\text { (Tzatchkov et. al, 2014) } \\
\text { Desviación media }=\sqrt{\frac{\sum_{i=1}^{n_{n}}\left(p_{i}-p_{i D}\right)^{2}}{n_{n}}}\end{array}$ & mca & 1 \\
\hline & & $\begin{array}{l}\text { Exceso de presión } \\
\text { media }\end{array}$ & $\begin{array}{l}\text { Indicador del excedente de presión media en la red (Tzatchkov et. al, } \\
\qquad \text { 2014) } \\
\qquad \text { Exceso de presión }=\frac{\sum_{i=1}^{n_{n}} \alpha_{i} \cdot q_{i}}{\sum_{i=1}^{n_{n}} q_{i}} \text { con } \begin{cases}\alpha_{i}=0 & \text { si } h_{i} \leq h_{i D} \\
\alpha_{i}=h_{i}-h_{i D} & \text { si } h_{i}>h_{i D}\end{cases} \end{array}$ & mca & 2 \\
\hline & & $\begin{array}{l}\text { Déficit de presión } \\
\text { media }\end{array}$ & $\begin{array}{l}\text { Indicador del déficit medio de la presión en la red (Tzatchkov et. al, } \\
\qquad \text { 2014) } \\
\text { Déficit de presión }=\frac{\sum_{i=1}^{n_{n}} \beta_{i} \cdot q_{i}}{\sum_{i=1}^{n_{n}} q_{i}} \text { con } \begin{cases}\beta_{i}=0 & \text { si } h_{i} \geq h_{i D} \\
\beta_{i}=h_{i}-h_{i D} & \text { si } h_{i}<h_{i D}\end{cases} \end{array}$ & mca & 2 \\
\hline & & $\begin{array}{c}\text { Hydraulic } \\
\text { performance pressure } \\
\text { related }(\mathrm{HP})\end{array}$ & $\begin{array}{l}\text { La métrica HP (Coelho, 1997) es un indicador de importancia de la } \\
\text { demanda media según la demanda y el nivel de importancia de la } \\
\text { presión de cada nudo }\left(i_{p_{j}}\right) \\
\qquad H P=\frac{\sum_{j=1}^{n_{n}} i_{p_{j}} \cdot q_{j}}{\sum_{j=1}^{n_{n}} q_{j}}\end{array}$ & $\%$ & 1 \\
\hline & Criterio 2.2 & $\begin{array}{l}\text { Índice de protección } \\
\text { contra incendios (IPI) }\end{array}$ & $\begin{array}{c}\text { Porcentaje de caso de incendio dónde no se cumplen con las } \\
\text { condiciones mínimas impuestas en la norma }\end{array}$ & $\%$ & 1,5 \\
\hline
\end{tabular}




\begin{tabular}{|c|c|c|c|c|c|}
\hline Objetivos & $\begin{array}{l}\text { Criterios de } \\
\text { evaluación }\end{array}$ & Métricas & Definición & Unidad & Peso \\
\hline & \multirow[t]{2}{*}{ Resiliencia de la red } & Índice de fiabilidad & $\begin{array}{l}\text { Este indicador es introducido por Todini }(2000) \text {, y consiste en el ratio } \\
\text { entre la energía no disipada frente a la disponible para disipar, según } \\
\text { la siguiente ecuación: } \\
\qquad I_{r}=1-\frac{P_{\text {int }}}{P_{\max }}=1-\frac{P_{t o t}-P_{\text {ext }}}{P_{\max }}=\frac{\gamma \sum_{i=1}^{n_{n}} q_{i}\left(h_{i}-h_{i D}\right)}{P_{\text {tot }}-\gamma \sum_{i=1}^{n_{n}} q_{i} \cdot h_{i D}}\end{array}$ & - & 2 \\
\hline & & Índice de la robustez & $\begin{array}{l}\text { Este concepto es introducido por Prasad y Park (2014) e intenta } \\
\text { estudiar de manera combinada los efectos de la energía excedente } \\
\text { en el sistema y la robustez de las mallas de la red. } \\
\qquad I_{r n}=\frac{\gamma \sum_{i=1}^{n_{n}} C_{i} \cdot P_{\text {int }}}{P_{\max }}\end{array}$ & - & 2 \\
\hline & \multirow{2}{*}{$\begin{array}{c}\text { Criterio } 2.3 \\
\text { Energía disponible en } \\
\text { red }\end{array}$} & $\begin{array}{l}\text { Energía entregada a } \\
\quad \text { los usuarios }\end{array}$ & $\begin{array}{l}\text { Exceso de energía entrega a los usuarios en cada nudo de consumo } \\
\text { en forma de presión hidráulica }\end{array}$ & $\%$ & 1,5 \\
\hline & & $\begin{array}{l}\text { Eficiencia energética } \\
\text { dinámica }\end{array}$ & $\begin{array}{l}\text { Relación entre la potencia mínima a aportar por la presión en el nudo } \\
\text { de consumo y la potencia real aportada }\end{array}$ & - & 2 \\
\hline \multirow{4}{*}{$\begin{array}{l}\text { Objetivo } 3 \\
\text { Calidad del agua de la } \\
\text { red resultante }\end{array}$} & \multirow{2}{*}{$\begin{array}{l}\text { Criterio } 3.1 \\
\text { Tiempo de } \\
\text { permanencia }\end{array}$} & $\begin{array}{l}\text { Tiempo máximo de } \\
\text { permanencia }\end{array}$ & $\begin{array}{l}\text { Tiempo máximo de permanencia del agua en la red para todo el } \\
\text { periodo de simulación }\end{array}$ & $\mathrm{h}$ & 2 \\
\hline & & $\begin{array}{l}\text { Tiempo medio de } \\
\text { permanencia }\end{array}$ & $\begin{array}{l}\text { Tiempo medio de permanencia del agua en la red para todo el } \\
\text { periodo de simulación }\end{array}$ & $\mathrm{h}$ & 1 \\
\hline & \multirow{2}{*}{$\begin{array}{c}\text { Criterio } 3.2 \\
\text { Concentración de } \\
\text { cloro }\end{array}$} & $\begin{array}{l}\text { Concentración media } \\
\text { de cloro }\end{array}$ & $\begin{array}{l}\text { Concentración media de cloro en la red para todo el periodo de } \\
\text { simulación }\end{array}$ & ppm & 1 \\
\hline & & $\begin{array}{l}\text { Concentración } \\
\text { mínima de cloro }\end{array}$ & $\begin{array}{l}\text { Concentración mínima de cloro en la red en cualquier instante del } \\
\text { periodo de simulación }\end{array}$ & ppm & 1,5 \\
\hline $\begin{array}{c}\text { Objetivo } 4 \\
\text { Coste de Implantación }\end{array}$ & $\begin{array}{l}\text { Criterio } 4.1 \\
\text { Costes directos de } \\
\text { implantación }\end{array}$ & $\begin{array}{l}\text { Costes directos de } \\
\text { implantación }\end{array}$ & $\begin{array}{l}\text { Costes directos de implantación de la técnica divido por la longitud de } \\
\text { red afectada }\end{array}$ & $\begin{array}{l}€ / \mathrm{km} \\
\text { red }\end{array}$ & 2 \\
\hline
\end{tabular}




\begin{tabular}{|c|c|c|c|c|}
\hline Objetivos & $\begin{array}{c}\text { Criterios de } \\
\text { evaluación }\end{array}$ & Métricas & Definición \\
\hline & $\begin{array}{c}\text { Criterio } 4.2 \\
\text { Otros costes }\end{array}$ & Otros costes & $\begin{array}{c}\text { Otros costes necesarios para el correcto funcionamiento o mejora de } \\
\text { la técnica seleccionada }\end{array}$ \\
\hline
\end{tabular}

Tabla 5.4 Métricas y Rangos de evaluación

\begin{tabular}{|c|c|c|c|c|c|c|}
\hline \multirow{2}{*}{ Objetivos } & \multirow{2}{*}{ Criterios de evaluación } & \multirow{2}{*}{ Métricas } & \multirow{2}{*}{ Unidad } & \multicolumn{3}{|c|}{ Rangos de evaluación } \\
\hline & & & & Bueno & Regular & Malo \\
\hline \multirow{2}{*}{$\begin{array}{c}\text { Objetivo } 1 \\
\text { Efectividad y eficiencia en } \\
\text { la búsqueda de fugas no } \\
\text { declaradas }\end{array}$} & $\begin{array}{l}\text { Criterio } 1.1 \\
\text { Coste anual campañas } \\
\text { búsqueda }\end{array}$ & Variación en el coste anual & $\%$ & $100-50$ & $50-10$ & $10-0$ \\
\hline & $\begin{array}{c}\text { Criterio } 1.2 \\
\text { Tiempo de ubicación }\end{array}$ & Velocidad de inspección & m/día & $2.000-1.000$ & $1.000-300$ & $300-0$ \\
\hline \multirow{9}{*}{$\begin{array}{l}\text { Objetivo } 2 \\
\text { Capacidad hidráulica de la } \\
\text { red resultante }\end{array}$} & \multirow{4}{*}{$\begin{array}{l}\text { Criterio } 2.1 \\
\text { Presión hidráulica }\end{array}$} & Variabilidad de la presión & mca & $0-20$ & $20-40$ & $40-100$ \\
\hline & & Exceso de presión media & mca & $0-10$ & $10-20$ & $20-30$ \\
\hline & & Déficit de presión media & mca & $0--2$ & $-2--6$ & $-6--10$ \\
\hline & & $\begin{array}{l}\text { Hydraulic performance pressure } \\
\text { related (HP) }\end{array}$ & $\%$ & $3-2$ & $2-1$ & $1-0$ \\
\hline & \multirow{3}{*}{$\begin{array}{c}\text { Criterio } 2.2 \\
\text { Resiliencia de la red }\end{array}$} & $\begin{array}{l}\text { Índice de protección contra incendios } \\
\text { (IPI) }\end{array}$ & $\%$ & $0-5$ & $5-10$ & $10-20$ \\
\hline & & Índice de fiabilidad & - & $1-0,67$ & $0,67-0,33$ & $0,33-0$ \\
\hline & & Índice de la robustez & - & $1-0,67$ & $0,67-0,33$ & $0,33-0$ \\
\hline & \multirow[t]{2}{*}{$\begin{array}{l}\text { Criterio } 2.3 \\
\text { Energía disponible en red }\end{array}$} & $\begin{array}{c}\text { Exceso de energía entregada a los } \\
\text { usuarios }\end{array}$ & $\%$ & $0-70$ & $70-150$ & $150-300$ \\
\hline & & Eficiencia energética dinámica & $\%$ & $100-60$ & $60-40$ & $40-0$ \\
\hline
\end{tabular}




\begin{tabular}{|c|c|c|c|c|c|c|}
\hline \multirow{2}{*}{ Objetivos } & \multirow{2}{*}{ Criterios de evaluación } & \multirow{2}{*}{ Métricas } & \multirow{2}{*}{ Unidad } & \multicolumn{3}{|c|}{ Rangos de evaluación } \\
\hline & & & & Bueno & Regular & Malo \\
\hline \multirow{4}{*}{$\begin{array}{l}\text { Objetivo } 3 \\
\text { Calidad del agua de la red } \\
\text { resultante }\end{array}$} & \multirow{2}{*}{$\begin{array}{c}\text { Criterio } 3.1 \\
\text { Tiempo de permanencia }\end{array}$} & Tiempo máximo de permanencia & $\mathrm{h}$ & $0-8$ & $8-16$ & $16-24$ \\
\hline & & Tiempo medio de permanencia & $\mathrm{h}$ & $0-8$ & $8-16$ & $16-24$ \\
\hline & \multirow{2}{*}{$\begin{array}{c}\text { Criterio } 3.2 \\
\text { Concentración de cloro }\end{array}$} & Concentración media de cloro & ppm & $1-0,7$ & $0,7-0,4$ & $0,4-0$ \\
\hline & & Concentración mínima de cloro & ppm & $1-0,7$ & $0,7-0,4$ & $0,4-0$ \\
\hline \multirow{2}{*}{$\begin{array}{c}\text { Objetivo } 4 \\
\text { Coste de Implantación }\end{array}$} & $\begin{array}{l}\text { Criterio } 4.1 \\
\text { Costes directos de } \\
\text { implantación }\end{array}$ & Costes directos de implantación & $€ / \mathrm{km}$ red & $0-1.500$ & $1.500-3.000$ & $3.000-5.000$ \\
\hline & $\begin{array}{l}\text { Criterio } 4.2 \\
\text { Otros costes }\end{array}$ & Otros costes & $€ / \mathrm{km}$ red & $0-400$ & $400-800$ & $800-1.500$ \\
\hline
\end{tabular}




\subsection{Caso de estudio}

\subsubsection{Caso práctico 1. Red sintética}

\subsubsection{Planteamiento del caso}

El primer caso de estudio consiste en la red sintética empleada en los capítulos 3 y 4 para abordar las metodologías planteadas. Se trata de un sistema con una longitud total de $50 \mathrm{~km}$ con 58 tuberías que suministran 38 nudos de consumo cuya elevación es cero. Alrededor de $6.500 \mathrm{~m}^{3}$ de agua se entregan diariamente (40.000 habitantes), e idealmente se supone que toda el agua es registrada.

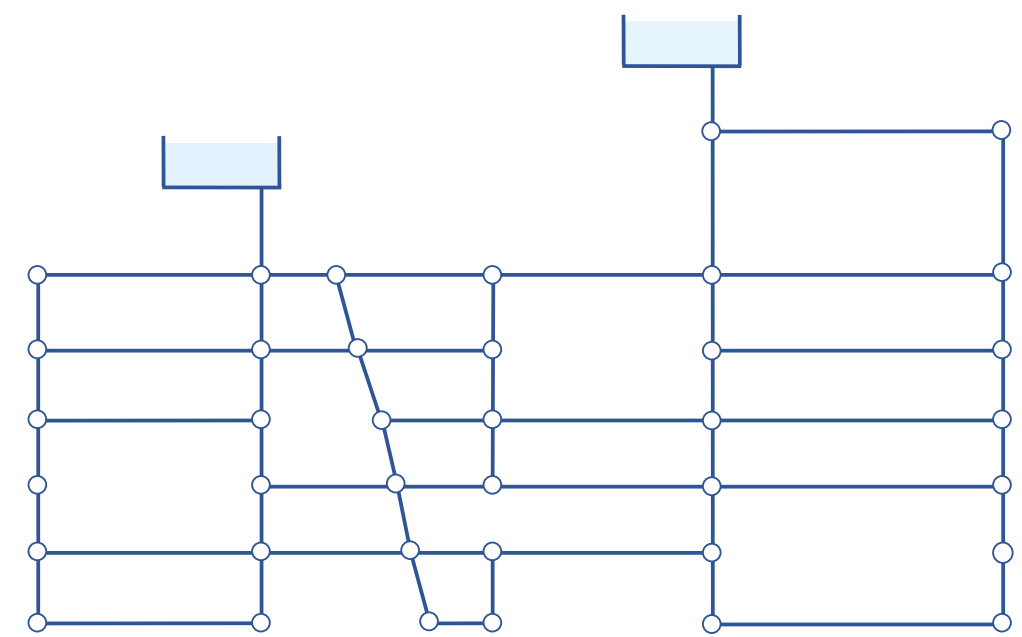

Figura 5.3 Red de distribución del caso 1

Tabla $5.1 \quad$ Características descriptivas del sistema del caso 1

\begin{tabular}{|c|c|c|c|c|c|c|c|c|c|}
\hline \multirow{2}{*}{ Sector } & \multicolumn{2}{|c|}{ Longitud } & \multirow{2}{*}{$\begin{array}{c}\text { Diámetro } \\
\text { de } \\
\text { suministro }\end{array}$} & \multirow{2}{*}{$\begin{array}{c}\text { Gestión } \\
\text { de } \\
\text { presión }\end{array}$} & \multicolumn{2}{|c|}{$\begin{array}{c}\text { Volumen } \\
\text { suministrado }\end{array}$} & \multicolumn{2}{|c|}{$\begin{array}{l}\text { Volumen } \\
\text { registrado }\end{array}$} & \multirow{2}{*}{$\begin{array}{l}\text { № de } \\
\text { nudos }\end{array}$} \\
\hline & $(\mathrm{km})$ & (\%) & & & $\mathrm{m}^{3} /$ día & $(\%)$ & m³/día & $(\%)$ & \\
\hline Red caso 1 & 50 & 100 & 200 & No & $6.566,4$ & 100 & $6.566,4$ & 100 & 38 \\
\hline
\end{tabular}

El comportamiento hidráulico de la red de estudio se obtiene con la ayuda de un modelo de simulación, elaborado en Epanet (Rossman, 2000). En su estado operativo normal, la presión promedio es de 37,7 mca, con valores extremos de 31,7 y 47,0 mca (Tabla 5.2 y Figura 5.5) frente a una presión mínima de servicio de $20 \mathrm{mca}$. La velocidad promedio del agua y la pérdida de carga unitaria en las tuberías son de $0,41 \mathrm{~m} / \mathrm{s}$ y $2,50 \mathrm{mca} / \mathrm{km}$, respectivamente (Tabla 3.14). La contribución del flujo para cada depósito es aproximadamente del 50\%, siendo el caudal promedio del agua en las tuberías de 6,65 l/s (399,25 l/min).

Tabla 5.2 Balance hídrico de la red

\begin{tabular}{|l|c|}
\hline & $\begin{array}{c}\text { Sistema } \\
\text { Completo }\end{array}$ \\
\hline Caudal suministrado (m³/día) & $6.566,4$ \\
\hline Presión media (mca) & 37,7 \\
\hline Rango de presiones (mca) & $31,7-47,0$ \\
\hline
\end{tabular}


El sistema cuenta con caudalímetros a la salida de los dos depósitos, cuyos registros se reciben de manera continua. Todos los puntos de consumo están equipados con contador, y cuenta con un sistema de telelectura de red fija el cual proporciona lecturas horarias de todos ellos.
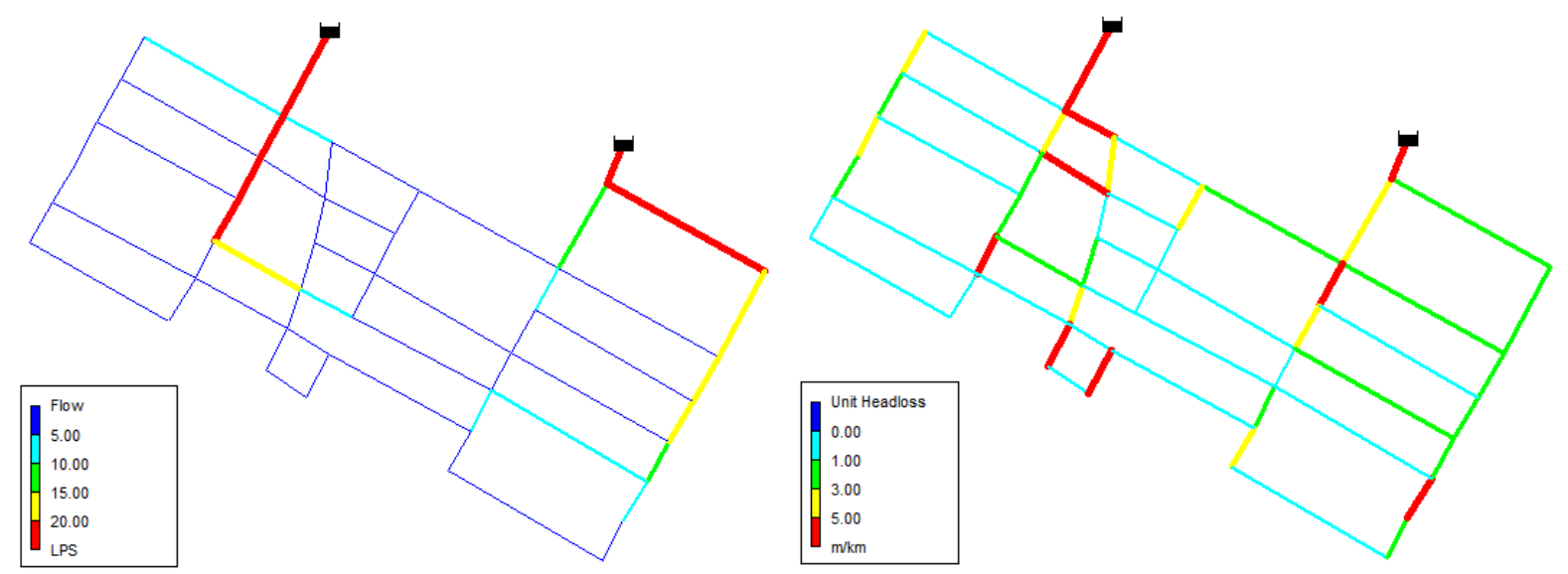

Figura 5.4 Caudales (izquierda) y pérdidas unitarias (derecha) en las tuberías

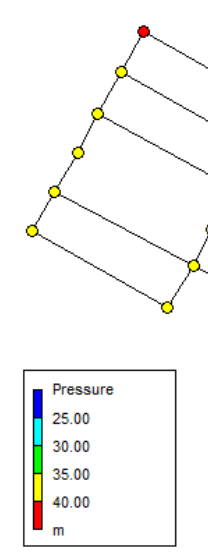

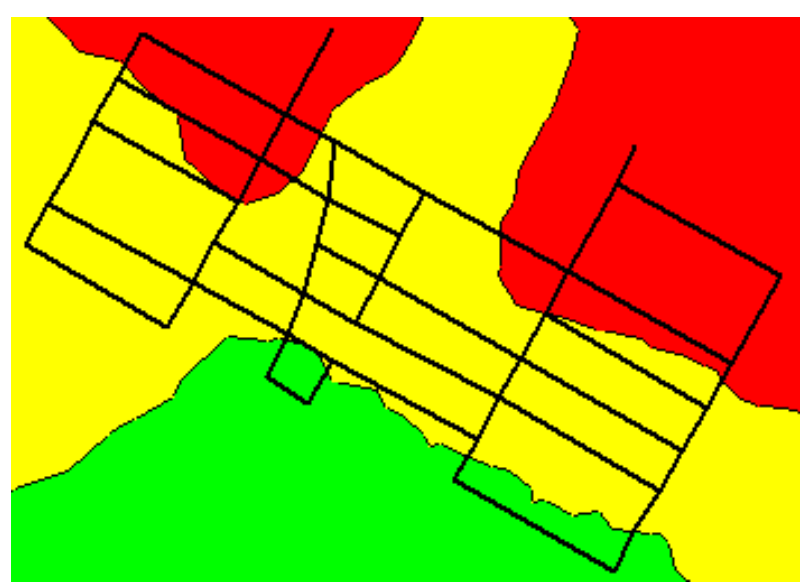

Presión en los nudos

Analizando energéticamente la red, bajo un escenario de comportamiento normal, se cuenta con una energía promedio introducida en el sistema de 894,72 kWh/día (Tabla 5.3). De los cuales el $75,24 \%$ se entrega en forma de presión en los puntos de consumo y en las fugas, mientras que el $24,76 \%$ restante se disipa durante el transporte debido a la fricción del agua con las tuberías. Todo ello, dota al sistema de una eficiencia energética dinámica del $53,16 \%$.

Tabla 5.3 Balance energético de la red del caso 1

\begin{tabular}{|c|c|c|c|c|c|c|c|c|}
\hline \multirow{2}{*}{ RED } & \multirow{2}{*}{$\begin{array}{c}\text { Demanda } \\
\left(\mathrm{m}^{3} / \mathrm{d}\right)\end{array}$} & \multicolumn{4}{|c|}{ TOTA ENERGÍA PERDIDA (kWh/d) } & \multirow{2}{*}{$\begin{array}{c}\text { Energía por } \\
\text { caudal } \\
\text { unitario } \\
\left(\mathrm{kWh} / \mathrm{m}^{3}\right)\end{array}$} & \multirow{2}{*}{$\begin{array}{l}\text { Mín. } \\
\text { energía en } \\
\text { grifo de } \\
\text { consumidor }\end{array}$} & \multirow{2}{*}{$\begin{array}{c}\text { Ef. } \\
\text { Energética } \\
\text { dinámica } \\
(\%)\end{array}$} \\
\hline & & Fricción & Control & $\begin{array}{c}\text { Grifo } \\
\text { consumidor }\end{array}$ & Total & & & \\
\hline $\begin{array}{l}\text { Sistema } \\
\text { completo }\end{array}$ & $6.566,4$ & 221,52 & 0 & 673,20 & 894,72 & 0,10 & 357,84 & 53,16 \\
\hline
\end{tabular}

Desde un punto de vista operativo, se sigue una estrategia de gestión de fugas basada en el método de los caudales nocturnos y una política de intervención proactiva. El nivel de fugas se monitorea continuamente y se toma acción tan pronto como se detecte una fuga en la red de distribución. La frecuencia de aparición de roturas sobre la red de distribución es de 
1,60 roturas $/ \mathrm{km} / \mathrm{año}$, donde el $50 \%$ de las fugas son no declaradas, y el tamaño medio de cada fuga está en $150 \mathrm{l} / \mathrm{min}$. Respecto a las fugas en acometida, se cuenta con una frecuencia media de 1 fugas $/ \mathrm{km}$ red.año, al igual que en el caso anterior, la mitad de las fugas en acometida son declaradas y el tamaño medio se estima en $27 \mathrm{l} / \mathrm{min}$. Se asume que se tarda en ubicar y reparar las fugas no declaradas en red un tiempo medio de 3 días, siendo de 14 días para las fugas en acometida. Respecto al caso de las declaradas, estos tiempos descienden a 1 y 7 días, respectivamente.

El coste medio del agua es de $0,24 € / \mathrm{m}^{3}$. Respecto a los costes de reparación de las fugas, se asume independiente de la estrategia de gestión de fuga seguida, y por lo tanto no se tendrá presente para los análisis posteriores. Para cada sector, el coste anual de equipos y mantenimiento es de 1.087,80 €/año, según el siguiente detalle: un coste inicial de $18.000 €$ por sector (independientemente del tamaño de este), una vida útil de todos los equipos de 10 años, un coste anual de mantenimiento asumido en un $20 \%$ sobre el coste inicial y una tasa de descuento del 5\%. El equipo de correlación y escucha supondrá un coste anual de 95,14 $€ / \mathrm{km}$ red y año, según el siguiente destalle: coste inicial de $39.000 €$ por equipo de trabajo, una vida útil de todos los equipos de 5 años, el coste de mantenimiento se asume en un $20 \%$ sobre el coste inicial y una tasa de descuento del $5 \%$. Cada equipo de trabajo estará formado por un solo operario con un salario anual de $40.000 €$ de coste más un $50 \%$ en concepto de gastos generales. El rendimiento de trabajo de correlación y escucha será de 300 metros revisados a la hora, con jornadas de 8 horas.

\subsubsection{Sectorización propuesta según conceptos energéticos}

Una vez conocida la red de partida, y de cara a realizar una comparación entre metodologías, es necesario definir cuáles serán los diferentes escenarios de actuación.

El primer escenario consistirá en la sectorización planteada en el apartado 3.3.2, del capítulo 3. Se trata de un diseño basado en criterios energéticos sin restricciones externas impuestas por el diseñador. Como resultado, la red ha quedado dividida en 4 sectores hidráulicos independientes cuyas características se recogen en la tabla Tabla 5.4. El sector 1, sombreado en rojo en la Figura 5.6, engloba el $20,1 \%$ de la longitud de la red y un total de 6 nudos de consumo. El segundo sector, que se encuentra sombreado en azul, está compuesto por el $20,1 \%$ de las tuberías y 8 nudos. El sector 3, en naranja, está formado por el $30 \%$ de las tuberías y 10 nudos de consumo. Finalmente, el sector 4, sombreado en verde, engloba el $30,8 \%$ de las tuberías y 14 nudos. Para su ejecución, ha sido necesaria la instalación de 3 contadores sectoriales y 9 válvulas de corte. No se cuenta con gestión de presión en ninguno de los sectores. Respecto al reparto del volumen suministrado, el sector 1 demanda el 13,2\% del volumen total entregado al sistema empleando para ello una tubería de $200 \mathrm{~mm}$, el sector 2 requiere 10,5\% del total y emplea una tubería del mismo calibre, al sector 3 se le suministra el $36,8 \%$ del volumen inyectado al sistema a través de una tubería de $200 \mathrm{~mm}$, siendo el $39,5 \%$ restante suministrado al sector 4 . En este caso, el sector cuenta con dos puntos de suministro de 90 y $200 \mathrm{~mm}$. Como se apuntó en el planteamiento del caso, todo el volumen suministrado es registrado y consumido, para no dotar de mayor complejidad al caso de estudio 1. 
Tabla 5.4 Características descriptivas de los sectores según criterios energéticos

\begin{tabular}{|c|c|c|c|c|c|c|c|c|c|}
\hline \multirow{2}{*}{ Sector } & \multicolumn{2}{|c|}{ Longitud } & \multirow{2}{*}{$\begin{array}{l}\text { Diámetro } \\
\text { de } \\
\text { suministro }\end{array}$} & \multirow{2}{*}{$\begin{array}{l}\text { Gestión } \\
\text { de presión }\end{array}$} & \multicolumn{2}{|c|}{$\begin{array}{c}\text { Volumen } \\
\text { suministrado }\end{array}$} & \multicolumn{2}{|c|}{$\begin{array}{c}\text { Volumen } \\
\text { registrado }\end{array}$} & \multirow{2}{*}{$\begin{array}{l}\text { № de } \\
\text { nudos }\end{array}$} \\
\hline & $(\mathrm{km})$ & (\%) & & & $\mathrm{m}^{3} /$ día & $(\%)$ & $\mathrm{m}^{3} /$ día & $(\%)$ & \\
\hline 1 - rojo & 10,2 & 20,1 & 200 & No & 864 & 13,2 & 864 & 13,2 & 6 \\
\hline 2 - azul & 10,2 & 20,1 & 200 & No & 691 & 10,5 & 691 & 10,5 & 8 \\
\hline 3 - naranja & 15,2 & 30,0 & 200 & No & 2419 & 36,8 & 2419 & 36,8 & 10 \\
\hline 4 - verde & 15,6 & 30,8 & $90+200$ & No & 2592 & 39,5 & 2592 & 39,5 & 14 \\
\hline
\end{tabular}

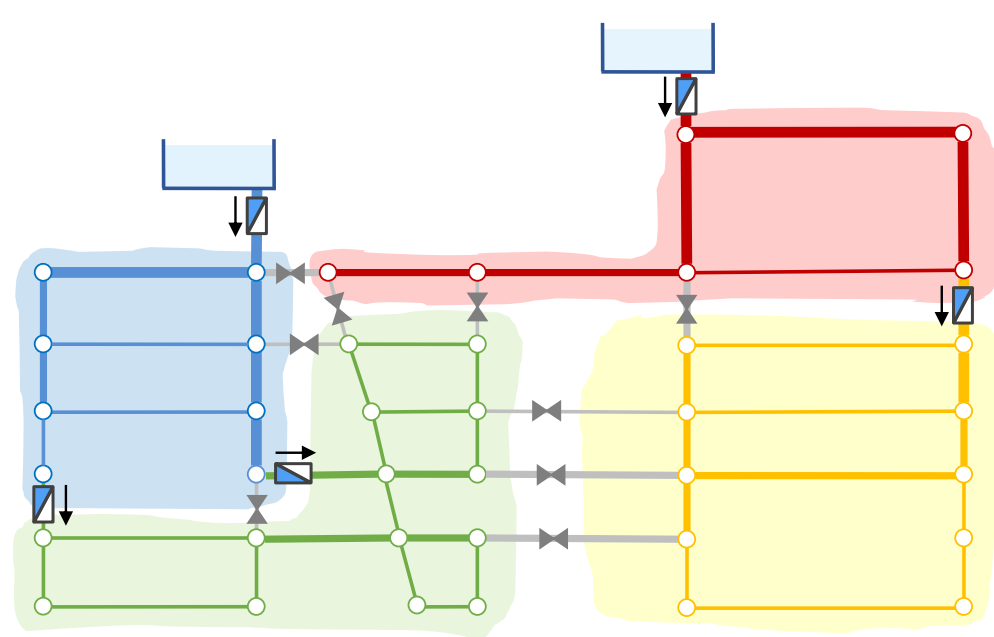

Figura 5.6 Sectorización final propuesta según criterios energéticos

Analizando el comportamiento hidráulico de este nuevo escenario, en su estado operativo normal, se cuenta con una presión media de 31,20 mca, con valores externos de 20,05 y 47,02 mca (Figura 5.8), frente a una presión mínima de servicio de 20 mca. La velocidad promedio del agua y la pérdida de carga unitaria en las tuberías son de 0,45 m/s y 3,47 mca/km, respectivamente (Figura 5.7).
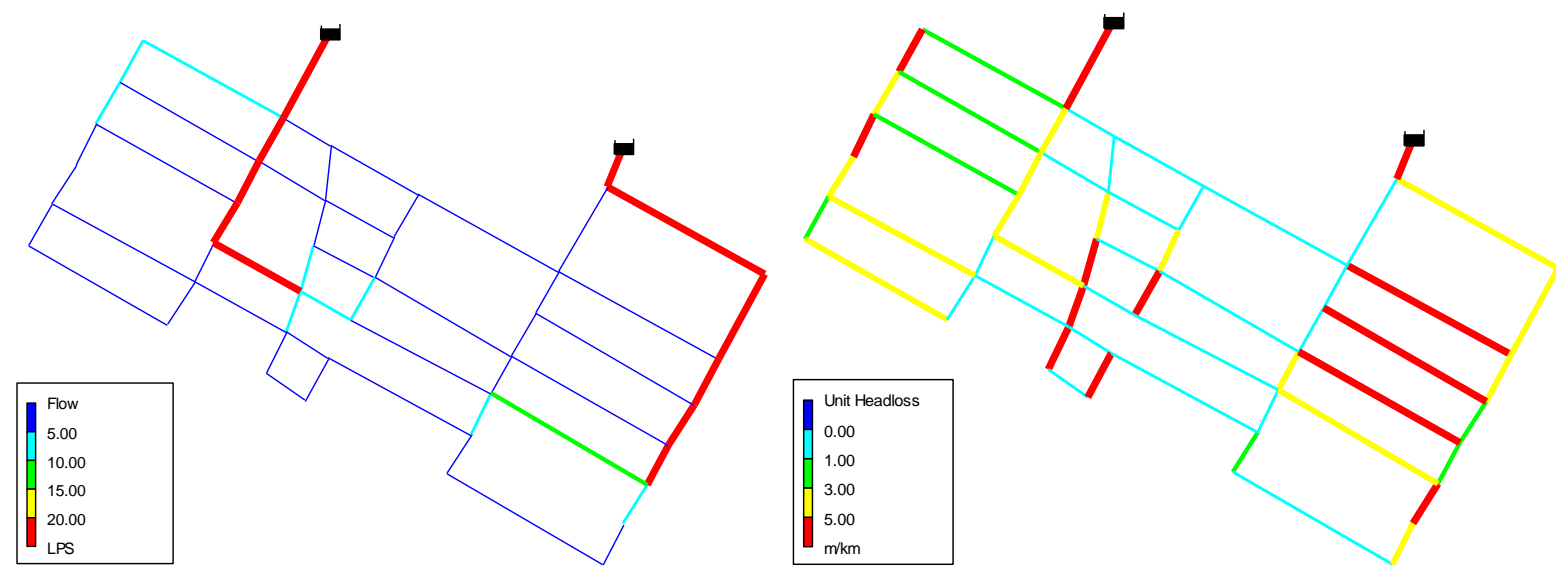

Figura 5.7 Caudales (izquierda) y pérdidas unitarias (derecha) bajo la sectorización propuesta 

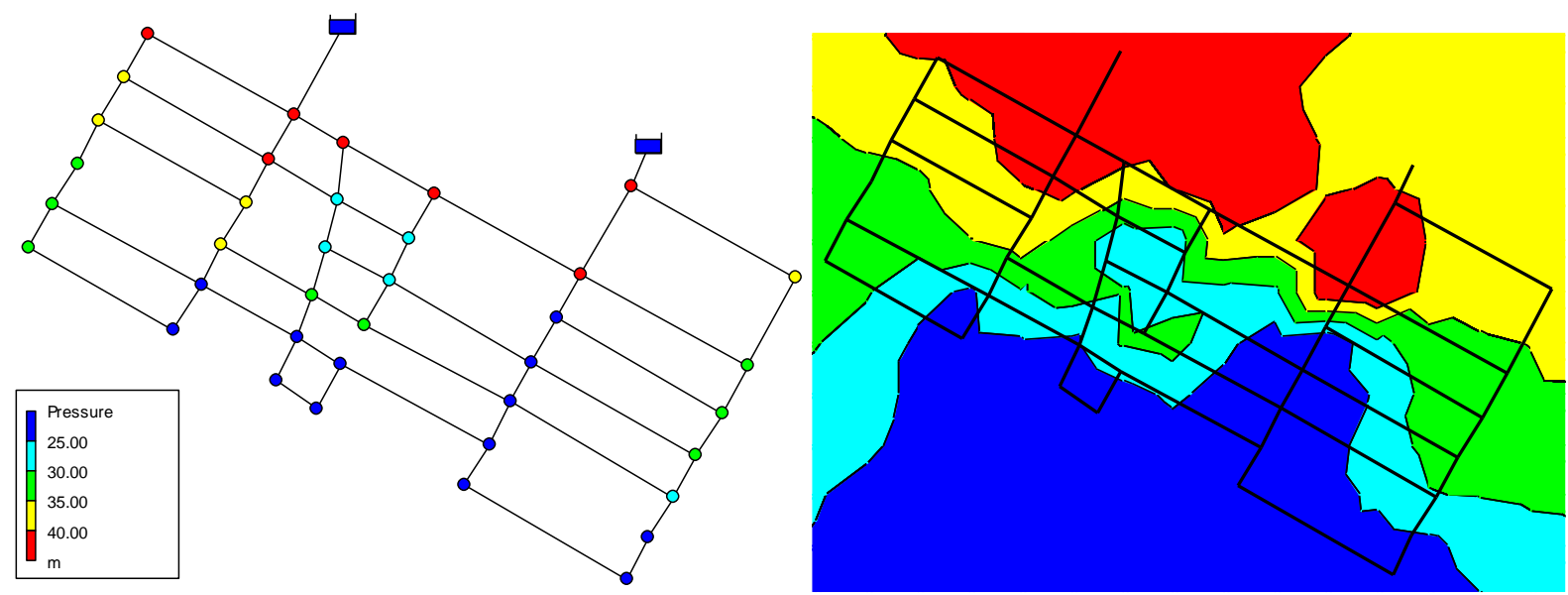

Figura 5.8 Nivel de presiones en los nudos bajo la sectorización propuesta

Entrando en mayor grado de detalle, se desprende una gran diferencia entre sectores. Se puede observar unos niveles algo inferiores en los sectores 3 y 4 , debido al efecto que introducen en el sistema el cierre de tuberías. En la Tabla 5.5 se recogen los principales indicadores hidráulicos de la red completa y de cada uno de los sectores.

Tabla 5.5 Balance hídrico de la red y de cada uno de los sectores según criterios energéticos

\begin{tabular}{|l|c|c|c|c|c|}
\hline & $\begin{array}{c}\text { Sistema } \\
\text { Completo }\end{array}$ & Sector 1 & Sector 2 & Sector 3 & Sector 4 \\
\hline Caudal suministrado (m³/día) & $6.566,4$ & 864,0 & 691,2 & $2.419,2$ & $2.592,0$ \\
\hline Presión media (mca) & 31,2 & 42,7 & 38,7 & 24,8 & 26,6 \\
\hline Rango presiones (mca) & $20,1-47,0$ & $34,5-47,0$ & $33,1-43,2$ & $20,9-32,5$ & $20,1-33,6$ \\
\hline
\end{tabular}

Desde el punto de vista energético, bajo un escenario de comportamiento normal, se entrega al sistema un total de $894,72 \mathrm{kWh} / \mathrm{d}$ (Tabla 5.6). De los cuales el $58,8 \%$ se localiza en los puntos de consumo en forma de presión y el $41,2 \%$ restante se disipa durante el transporte debido a la fricción del agua con las tuberías. Todo ello, dota al sistema sectorizado según criterios energéticos de una eficiencia energética dinámica del 68,02\%. Entrando en detalle en cada uno de los sectores, se puede comprobar como los sectores 3 y 4 presentan unos valores de eficiencia mayores, derivado de la reducción de presión en estas zonas por el cierre de tuberías. Otro aspecto para destacar es la energía perdida por fricción en los sectores 1 y 2. Se puede comprobar cómo el valor es mayor en cada uno de ellos. Esto es debido a que por el sector 1 circula el volumen suministrado al sector 3 y por el sector 2 circula el agua suministrada al sector 4. Esto hace que, en los sectores cabecera, y directamente proporcional al caudal trasegado, la pérdida de carga por fricción sea mayor.

Tabla 5.6 Balance energético de la red con fugas tras la sectorización según criterios energéticos

\begin{tabular}{|c|c|c|c|c|c|c|c|c|}
\hline \multirow[b]{2}{*}{ RED } & \multirow{2}{*}{$\begin{array}{c}\text { Demanda } \\
\left(\mathrm{m}^{3} / \mathrm{d}\right)\end{array}$} & \multicolumn{4}{|c|}{ TOTA ENERGÍA PERDIDA (kWh/d) } & \multirow{2}{*}{$\begin{array}{l}\text { Energía } \\
\text { por caudal } \\
\text { unitario } \\
\left(\mathrm{kWh} / \mathrm{m}^{3}\right)\end{array}$} & \multirow{2}{*}{$\begin{array}{l}\text { Mín. } \\
\text { energía en } \\
\text { grifo de } \\
\text { consumidor }\end{array}$} & \multirow{2}{*}{$\begin{array}{c}\text { Ef. } \\
\text { Energética } \\
\text { dinámica } \\
(\%)\end{array}$} \\
\hline & & Fricción & Control & $\begin{array}{c}\text { Grifo } \\
\text { consumidor }\end{array}$ & Total & & & \\
\hline $\begin{array}{l}\text { Sectorización } \\
\text { energética }\end{array}$ & 6566,40 & 368,64 & - & 526,08 & 894,72 & 0,08 & 357,84 & 68,02 \\
\hline Sector 1 & 864,00 & 119,52 & - & 100,56 & 220,08 & 0,12 & 47,04 & 46,82 \\
\hline Sector 2 & 691,20 & 115,44 & - & 72,96 & 188,16 & 0,11 & 37,68 & 51,71 \\
\hline
\end{tabular}




\begin{tabular}{|c|c|c|c|c|c|c|c|c|}
\hline \multirow[b]{2}{*}{ RED } & \multirow[b]{2}{*}{$\begin{array}{c}\text { Demanda } \\
\left(\mathrm{m}^{3} / \mathrm{d}\right)\end{array}$} & \multicolumn{4}{|c|}{ TOTA ENERGÍA PERDIDA (kWh/d) } & \multirow{2}{*}{$\begin{array}{c}\text { Energía } \\
\text { por caudal } \\
\text { unitario } \\
\left(\mathrm{kWh} / \mathrm{m}^{3}\right)\end{array}$} & \multirow{2}{*}{$\begin{array}{l}\text { Mín. } \\
\text { energía en } \\
\text { grifo de } \\
\text { consumidor }\end{array}$} & \multirow{2}{*}{$\begin{array}{c}\text { Ef. } \\
\text { Energética } \\
\text { dinámica } \\
(\%)\end{array}$} \\
\hline & & Fricción & Control & $\begin{array}{c}\text { Grifo } \\
\text { consumidor }\end{array}$ & Total & & & \\
\hline Sector 3 & 2419,20 & 66,72 & - & 160,56 & 227,28 & 0,07 & 131,76 & 82,15 \\
\hline Sector 4 & 2592,00 & 66,96 & - & 192,24 & 259,2 & 0,07 & 141,36 & 73,51 \\
\hline
\end{tabular}

\subsubsection{Sensorización para la localización de fugas}

El segundo escenario que se plantea se centra en el uso de sensores para la localización de los consumos incontrolados producidos a lo largo de la red, principalmente fugas, idea desarrollada en el apartado 4.5.

Para la aplicación de dicha metodología, se parte de la red de distribución en condiciones normales de funcionamiento. Sobre ésta se produce un volumen incontrolado de magnitud medible, pero de localización desconocida y se resuelven las ecuaciones de masa y energía, pero en este caso, tomando como valores conocidos las presiones en todos los nudos para ese nuevo escenario, y como incógnitas los caudales circulantes y los consumos adicionales en cada nudo. Como resultado, se cuantifica el consumo adicional para cada nudo, y, en consecuencia, la producción del volumen incontrolado.

Se debe conocer el valor de presión alcanzado en cada uno de los nudos cuando se produzca la citada fuga. Para una pequeña parte de los nudos, aquellos equipados con sensores de presión, se conoce su valor real. Pero para el resto, será necesario estimarlo. En este caso, se realizará un análisis de sensibilidad (apartado 4.3) que proporciona la variación de presión media sufrida en cada nudo cuando se produzca una fuga de un valor predeterminado en cualquier punto de la red. Es decir, a la hora de estimar el valor de presión de un nudo cuando se está produciendo una fuga, en cualquier punto de la red, se considera que el menor error introducido se producirá al estimar la presión media sufrida por ubicar la fuga en todas las ubicaciones posibles. Esta presión media se puede obtener realizando $n$ simulaciones, tantas como nudos, con la fuga ubicada en un punto diferente en cada una de ellas. Aunque debido al alto coste computacional que ello supone, se propone obtenerlo de manera directa con el modelo de análisis de sensibilidad ya propuesto. Este cálculo se puede extrapolar a los caudales en las tuberías, obteniendo de igual manera la variación del flujo en cada tubería para un aumento localizado conocido.

Para aplicar dicha metodología es necesario conocer qué caudal se está escapando del sistema sin ser controlado, que en este caso se fija en $30 \mathrm{l} / \mathrm{min}$, y qué red de sensores se tienen instalados, así como sus lecturas registradas. Con el objetivo de analizar el alcance de esta metodología de búsqueda para este caso de estudio, se analizan diferentes distribuciones de sensores de presión y caudal en el capítulo 4. Aunque para este caso de estudio se han elegido un escenario con 15 sensores de presión homogéneamente distribuidos.

Una vez conocidos los datos de partida, y como primer paso, se realizará un análisis de sensibilidad para este escenario. Como resultado se obtendrá la variación media sufrida en la altura piezométrica de los nudos y los caudales circulantes por las tuberías. En el caso de los nudos, ante una fuga de $30 \mathrm{l} / \mathrm{min}$, se produce una disminución media en la presión de todos los nudos con un valor medio de 0,17 mca con una desviación de 0,09 mca (Figura 5.9). Se puede observar como la mayor parte de los valores obtenidos siguen el valor medio marcado, 
alejándose más o menos según la ubicación final del nudo. Aun así, se pueden observar varios nudos cuyo comportamiento se aleja del valor medio. Es el caso de los nudos que se encuentran junto al depósito de cabecera, y por ello su valor de presión no se ve alterado, o los nudos 27 y 30 (mayor discrepancia con el resto), cuya disposición hace que el descenso de presión antes una fuga sean algo mayor.

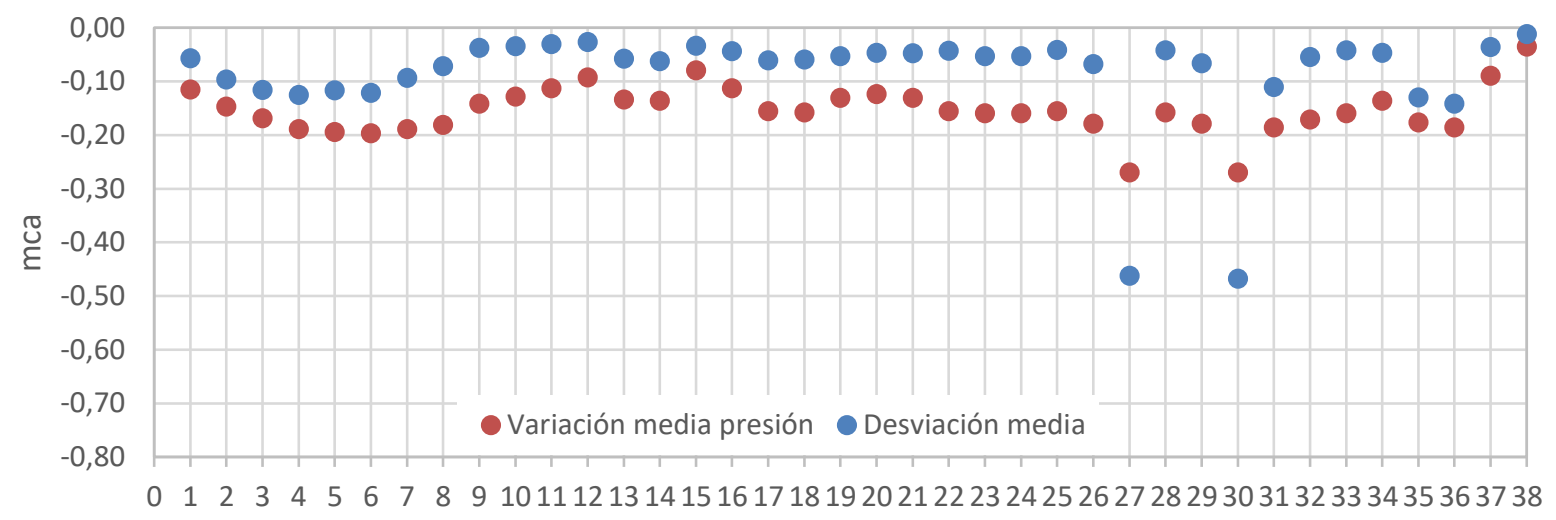

Figura 5.9 Variaciones medias de presión (mca) y sus desviaciones en los nudos ante una fuga de 30 $1 / \min$

En el caso de los caudales circulantes en las tuberías, en cambio, sí que se obtienen mayores discrepancias entre todas ellas (Figura 5.10), estando relacionada la variación sufrida con el nivel de caudal inicial, el cual está fuertemente relacionado con el diámetro de cada una de las tuberías. En el caso de los caudales, a diferencia de lo ocurrido en los nudos con la presión, no es del todo correcto hablar de valores medios obtenidos directamente del análisis de sensibilidad. Esto es debido a que los caudales vienen aparejados con un signo que informa sobre el sentido del flujo. Por lo tanto, una variación negativa del flujo significará que una disminución de este siempre y cuando el flujo inicial tenga un signo positivo. Es decir, mismos signos del flujo inicial y la variación significará que la tubería verá incrementado su caudal de manera media ante la aparición de un incremento en la red, y se verá reducido cuando los signos sean distintos. Teniendo presente está consideración, se puede apuntar que la variación del caudal medio en las tuberías es de 2,45 I/min con una desviación de 1,77, con valores que se mueven entre los $-0,62$ y $16,16 \mathrm{l} / \mathrm{min}$.

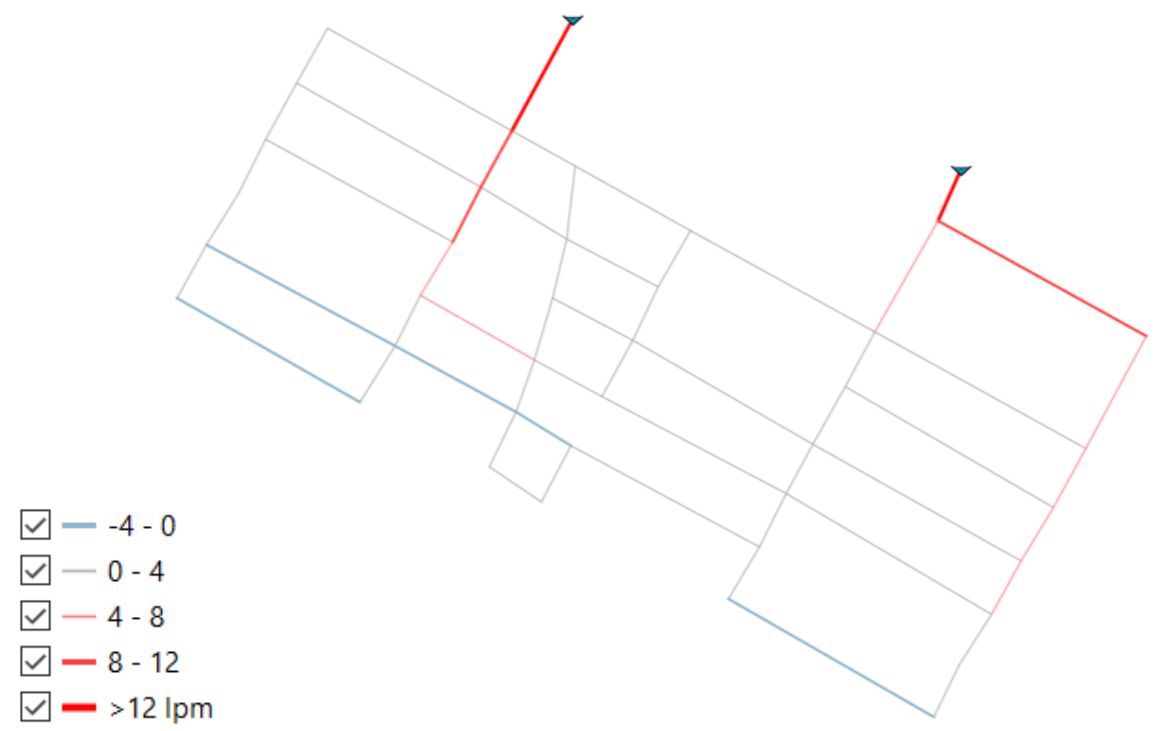

Figura 5.10 Variaciones de caudal (I/min), en valores absolutos, en las líneas ante una fuga de $30 \mathrm{l} / \mathrm{min}$ 
Un último aspecto por destacar, tras el análisis de la sensibilidad del flujo de las tuberías, es que una variación sufrida alta no tiene por qué llevar aparejada una desviación típica igualmente alta. Un buen ejemplo de ello es la tubería 1 (Tabla 5.7), que corresponde con la tubería que alimenta al sistema desde uno de los depósitos de cabecera. Sea cual sea la ubicación del exceso de demanda, el flujo de esta tubería siempre se verá incrementado en el mismo orden. De igual manera, se observan tuberías con variaciones medias bajas, pero con desviaciones típicas altas, como el caso de la tubería 41. Esto es debido a que esta tubería se ve muy afectada pero solo a unas determinadas ubicaciones de demanda, y muy poco por el resto.

Tabla 5.7 Tuberías con mayores variaciones de caudal (derecha) y desviaciones típicas (izquierda)

\begin{tabular}{|c|c|c|c|c|}
\hline Id & $\begin{array}{c}\text { Caudal } \\
\text { inicial } \\
(\mathrm{I} / \mathrm{min})\end{array}$ & $\begin{array}{c}\text { Caudal } \\
\text { promedio } \\
\text { con fuga de } \\
30 \mathrm{I} / \mathrm{min} \\
(\mathrm{I} / \mathrm{min})\end{array}$ & $\begin{array}{c}\text { Desviación } \\
\text { típica } \\
(\mathrm{I} / \mathrm{min})\end{array}$ & $\begin{array}{c}\text { Variación } \\
\text { sufrida } \\
(\mathrm{I} / \mathrm{min})\end{array}$ \\
\hline 41 & 155,89 & 155,27 & 7,05 & $-0,62$ \\
\hline 53 & $-129,62$ & $-131,82$ & 6,76 & 2,20 \\
\hline 14 & 531,82 & 535,59 & 6,58 & 3,78 \\
\hline 54 & 282,98 & 284,09 & 6,20 & 1,11 \\
\hline 38 & 1352,42 & 1360,03 & 6,13 & 7,61 \\
\hline 19 & $-856,29$ & $-861,24$ & 6,08 & 4,96 \\
\hline 42 & 109,51 & 108,89 & 5,94 & $-0,62$ \\
\hline 20 & $-936,86$ & $-942,73$ & 5,82 & 5,87 \\
\hline 51 & $-544,64$ & $-546,78$ & 5,82 & 2,14 \\
\hline 45 & 1025,49 & 1030,37 & 5,65 & 4,88 \\
\hline
\end{tabular}

\begin{tabular}{|c|c|c|c|c|}
\hline Id & $\begin{array}{c}\text { Caudal } \\
\text { inicial } \\
\text { (I/min) }\end{array}$ & $\begin{array}{c}\text { Caudal } \\
\text { promedio con } \\
\text { fuga de } 30 \\
\text { I/min (I/min) }\end{array}$ & $\begin{array}{c}\text { Desviación } \\
\text { típica } \\
(I / \text { min) }\end{array}$ & $\begin{array}{c}\text { Variación } \\
\text { sufrida } \\
(I / m i n)\end{array}$ \\
\hline 1 & 2297,61 & 2313,72 & 4,56 & 38,56 \\
\hline 7 & 2262,39 & 2276,28 & 4,56 & 37,94 \\
\hline 36 & 1561,69 & 1571,65 & 5,20 & 26,19 \\
\hline 37 & 1431,82 & 1440,49 & 5,58 & 24,01 \\
\hline 8 & 1341,66 & 1349,82 & 4,50 & 22,50 \\
\hline 38 & 1352,42 & 1360,03 & 6,13 & 22,67 \\
\hline 9 & 1161,66 & 1169,03 & 4,72 & 19,48 \\
\hline 21 & $-1127,69$ & $-1134,42$ & 5,35 & $-18,91$ \\
\hline 20 & $-936,86$ & $-942,73$ & 5,82 & $-15,71$ \\
\hline 19 & $-856,29$ & $-861,24$ & 6,08 & $-14,35$ \\
\hline
\end{tabular}

Una vez realizado el análisis de sensibilidad, y obtenidas las variaciones medias de presión y caudal en los nudos y tuberías, el siguiente paso es obtener las lecturas de los sensores para poder realizar la localización de la fuga.

Para este caso, se contará inicialmente con una distribución de 15 sensores de presión ubicados de manera homogénea por la red (Figura 5.11). Se ha elegido una distribución homogénea de los mismos según los resultados del análisis de sensibilidad, donde se observaba un comportamiento similar de todos ellos. 


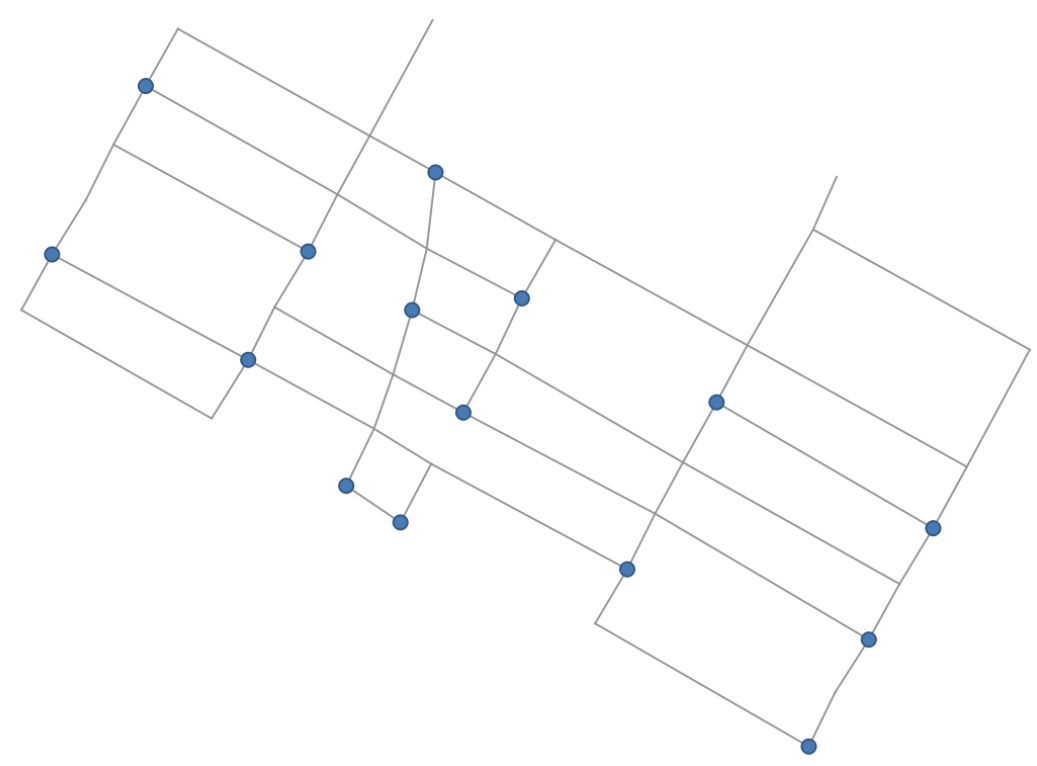

Figura 5.11 Distribución homogénea de los 15 sensores de presión

Los datos de presión recogidos por estos 15 sensores son datos reales, y entrarán en el modelo de cálculo como valores conocidos. El resto de los valores de presión, que también deben establecerse como valores conocidos, se estimarán como el valor del escenario inicial más la variación de presión media sufrida en cada nudo para el incremento de consumo fijado.

En el caso de tener tantos sensores como puntos de consumo, se obtendrían todos los datos de presión reales y el cálculo de dónde se está produciendo el consumo incontrolado sería directo y preciso, obteniendo como resultado el caudal fugado en el nudo donde se esté produciendo y cero en el resto. Ahora bien, conforme se vaya reduciendo el número de sensores, se estará introduciendo mayores estimaciones y la localización de dicho consumo no será tan precisa y dependerá de dónde se esté produciendo.

Con todo ello, y a modo de ejemplo, se simula una fuga de $30 \mathrm{l} / \mathrm{min}$ en el nudo 9 (redondeado en verde en la Figura 5.12). Con los datos obtenidos por los sensores, se simula el modelo desarrollado en el capítulo 4, obteniendo como resultado el caudal fugado en cada nudo. En un caso ideal, donde se contará con un medidor de presión en cada punto de consumo, se obtendría un consumo extra tan solo en el nudo 9. Pero conforme se ve reducido el número de sensores, los errores debido a las hipótesis de cálculo van tomando relevancia y, con ello, la zona de localización aumenta. Actualmente tan solo se cuenta con 15 sensores de presión repartidos por la red, lo cual supone una cobertura del $39,47 \%$ del total de nudos. Con esta disposición, en la Figura 5.12 se muestra en cada nudo el caudal de fuga que desprende el modelo, despreciando aquellos cuyo valor asignado es cercano a cero. 


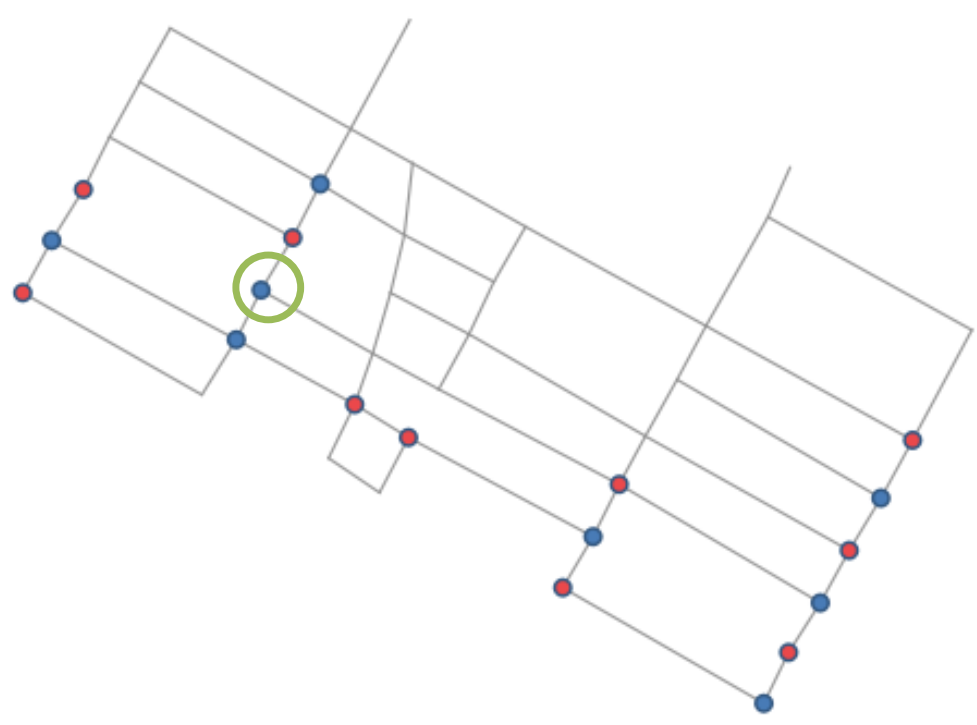

Figura 5.12 Ubicación real de la fuga (verde) y resultado del modelo de localización (azul y rojo)

A primera vista se podría considerar que la localización no ha sido la esperada, pero si analizamos con detalle los resultados obtenidos se puede comprobar que el modelo ha asignado a ciertos nudos (marcados en azul en la Figura 5.12) un caudal fugado negativo, es decir, en estos nudos se está produciendo una entrada de agua. Si analizamos alguno de ellos, se puede comprobar que el caudal de fuga que entra al sistema sale por los nudos colindantes. Esto es debido a que la presión introducida en el modelo por ciertos nudos es la real, al contar con un sensor de presión. Y la única manera de ajustar este valor con el valor de las presiones estimadas en sus nudos colindantes es asignando caudales consumidos negativos.

Ahora bien, aunque desde el punto de vista teórico sea admisible un caudal de fuga entrando al sistema, prácticamente no puede darse. Por ello, si solo destacamos aquellos nudos equipados con sensor, el resultado de la localización queda más claro. En la Figura $5.13 \mathrm{se}$ puede apreciar cómo, de todos los nudos equipados con sensores de presión, dos de los más cercano al nudo 9 marcan un consumo de fuga. Cuantitativamente, el modelo asigna un caudal fugado de 45,6 I/min al sensor más cercano y de $1,5 \mathrm{l} / \mathrm{min}$ al más alejado. Este valor está alejado de los $30 \mathrm{l} / \mathrm{min}$ de la fuga simulada, y la discrepancia vendrá marcada por la distancia entre la ubicación exacta y el sensor en cuestión. Aunque es importante destacar la importancia de obtener la localización de la zona donde se está produciendo la fuga, más que el valor en sí obtenido por el modelo. 


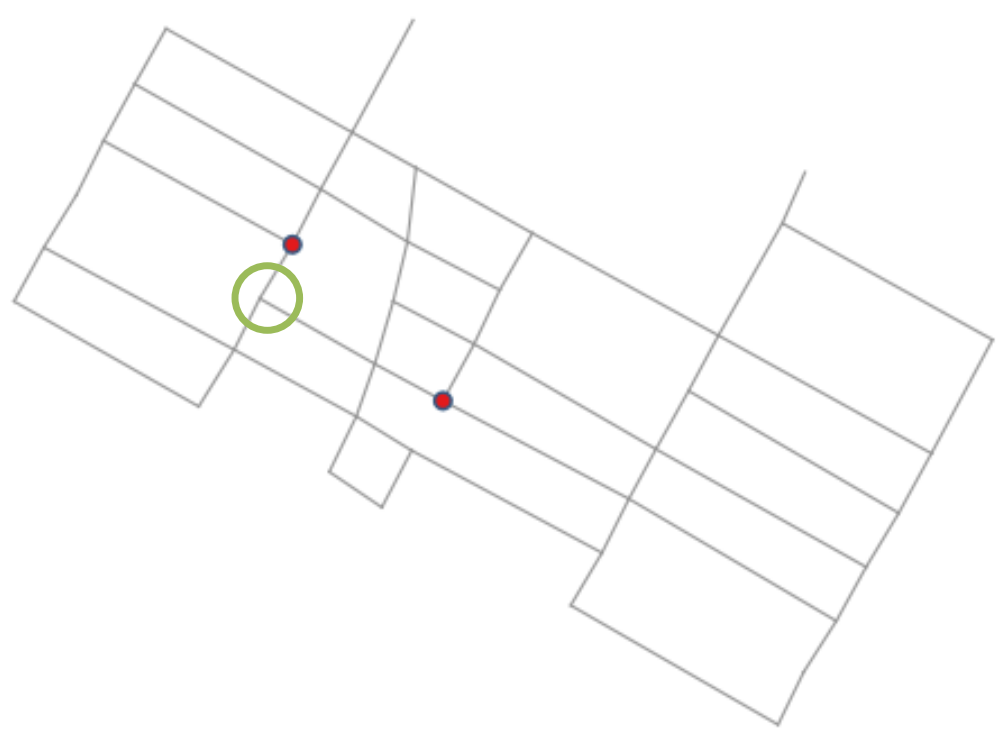

Figura 5.13 Ubicación real de la fuga (verde), nudo 9, y sensores con caudal fugado asignado (rojo)

Bajo el mismo escenario, el grado de acierto en la localización dependerá de la ubicación real de la fuga. Se muestra, como ejemplo, qué pasaría si la fuga se localizara en el nudo 4 (Figura 5.14) y en el nudo 33 (Figura 5.15). Esto demuestra que la facilidad en la localización depende en gran medida, no solo del número de sensores, sino de la ubicación de estos y la topología de la red.

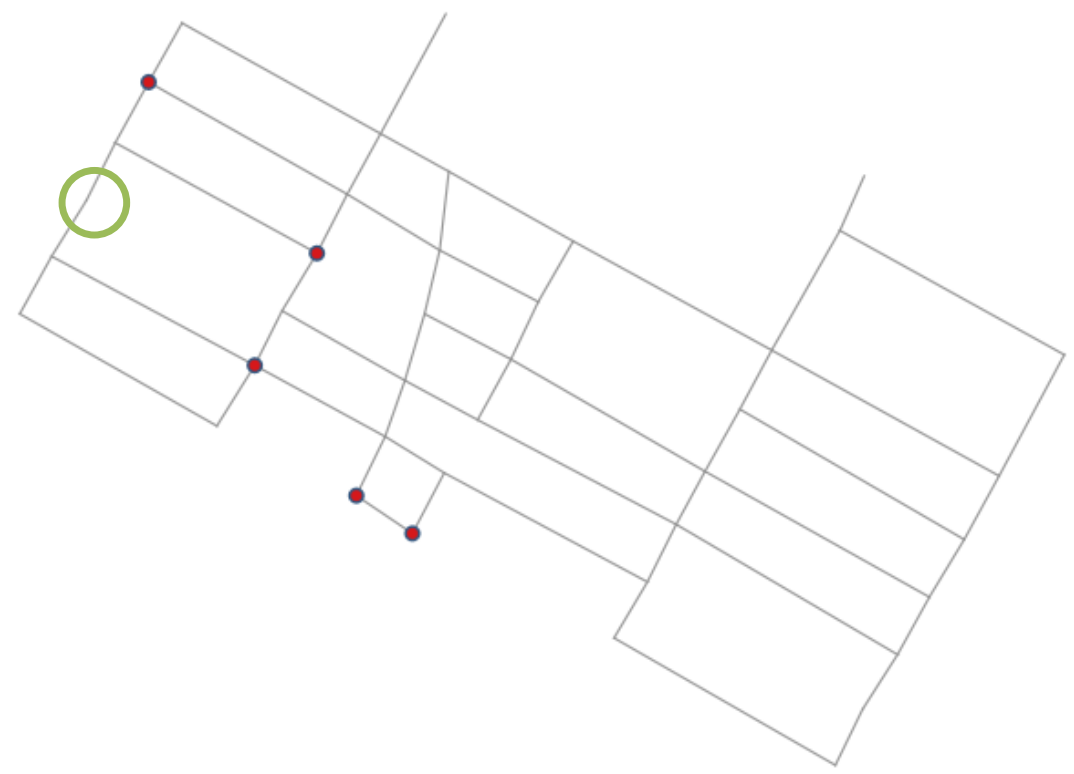

Figura 5.14 Ubicación real de la fuga (verde), nudo 4, y sensores con caudal fugado asignado (rojo) 


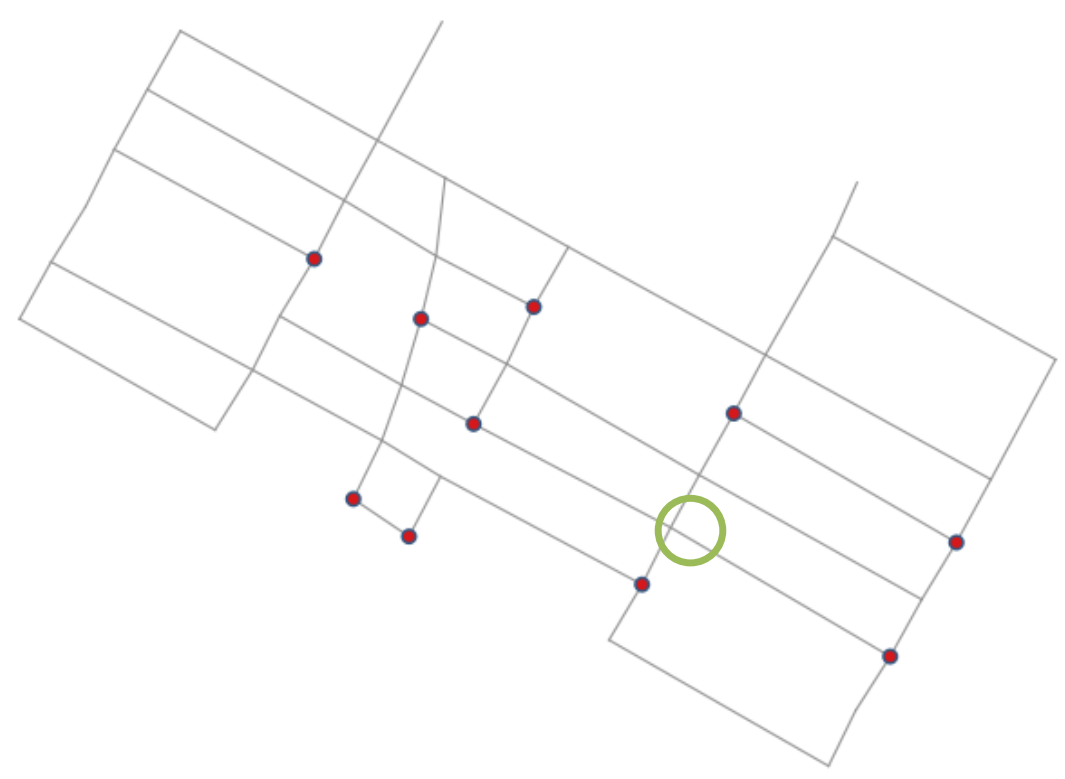

Figura 5.15 Ubicación real de la fuga (verde), nudo 33, y sensores con caudal fugado asignado (rojo)

Ahora bien, para analizar la fiabilidad de la metodología de localización sobre el escenario actual, es necesario simular todas las posibles ubicaciones, una por nudo, y analizar los resultados. Como se ha podido analizar a lo largo del caso, según la disposición y número de sensores y la topología de la red, como resultado con esta metodología se obtiene una zona de localización. Esta zona, medida en longitud de tubería, variará según dónde se produzca el consumo incontrolado. Así, para los 15 sensores disponibles y un caudal incontrolado de $30 \mathrm{l} / \mathrm{min}$, se obtiene una longitud de búsqueda promedio de 6,58 kilómetros (Tabla 5.8).

Tabla 5.8 Tabla resumen para la configuración de sensores probadas en el estudio de caso 1

\begin{tabular}{|c|c|}
\hline № se sensores de presión & $\begin{array}{c}\text { Escenario } \\
\text { estudiado }\end{array}$ \\
\hline № de caudalímetros & 15 \\
\hline Número escenarios & 0 \\
\hline $\begin{array}{c}\text { Ubicación de la fuga en el sensor } \\
\text { cercano }\end{array}$ & 38 \\
\hline Detección del área & 6 \\
\hline No detección & 36 \\
\hline Longitud de búsqueda promedio $(\mathrm{km})$ & 6,58 \\
\hline
\end{tabular}

\subsubsection{Efectividad y eficiencia en la búsqueda de fugas no declaradas}

Cuando se produce una fuga no declarada, el tiempo que transcurre desde que se origina hasta que se repara dependerá de la política de gestión de fugas seguida. En el presente caso de estudio, se sigue una política de intervención basada en la frecuencia de aparición de fugas en la red (Hunaidi, 2005) y se dispone de un equipo de búsqueda formado por un único operario.

Para el caso de estudio, se tienen los siguientes tiempos medios (Tabla 5.9) de detección para la política seguida, que coincidirá con la frecuencia de intervención, y el tiempo de correlación de la red para un único operario. 
Tabla 5.9 Tiempo de detección y correlación para la red inicial

\begin{tabular}{|c|c|c|c|}
\hline Escenario & $\begin{array}{c}\text { Frecuencia de } \\
\text { intervención (días) }\end{array}$ & $\begin{array}{c}\text { Tiempo de correlación } \\
\text { (jornadas) }\end{array}$ & $\begin{array}{c}\text { Velocidad de inspección } \\
\text { (m/h) }\end{array}$ \\
\hline Red inicial & 4,5 & 20,83 & 300 \\
\hline
\end{tabular}

Así, esta política de intervención supondrá un coste anual por las campañas de intervención y el agua fugada por un total de 230.049 €/año (Hunaidi, 2005), donde el 82,7\% corresponde a las campañas de búsqueda y el 17,3\% restante al coste del agua fugada.

Al sectorizar la red de distribución, la frecuencia de intervención global de toda la red pasará a calcularse para cada sector de manera individual. Así, y considerando la longitud de red englobada en cada uno de los sectores, se tendrán los valores recogidos en la Tabla 5.10.

Tabla 5.10 Frecuencia de intervención para cada uno de los sectores

\begin{tabular}{|c|c|}
\hline Sectores & $\begin{array}{c}\text { Frecuencia de } \\
\text { intervención (días) }\end{array}$ \\
\hline 1 & 24,7 \\
\hline 2 & 24,2 \\
\hline 3 & 19,4 \\
\hline 4 & 17,4 \\
\hline
\end{tabular}

Para dicho cálculo, se ha supuesto una distribución homogénea de las fugas en red, aunque una vez ejecutada la sectorización se permitirá el análisis de cada uno de los sectores y ajustar estas frecuencias de intervención.

Respecto a los tiempos de ubicación, estos dependerán de la longitud de cada sector, siendo para el caso que nos ocupa los recogidos en la Tabla 5.11.

Tabla 5.11 Tiempo de correlación para los sectores

\begin{tabular}{|c|c|c|}
\hline Sectores & $\begin{array}{c}\text { Tiempo de correlación } \\
\text { (jornadas) }\end{array}$ & $\begin{array}{c}\text { Velocidad de } \\
\text { inspección }(\mathbf{m} / \mathbf{h})\end{array}$ \\
\hline 1 & 3,8 & 300 \\
\hline 2 & 3,9 & 300 \\
\hline 3 & 4,8 & 300 \\
\hline 4 & 5,4 & 300 \\
\hline Media & 4,5 & 1.398 \\
\hline
\end{tabular}

Con todo ello, el coste asociado a esta política de gestión de fugas bajo la red sectorizada supondrá un total anual de $133.566 €$.

En el caso de usar la sensorización para la localización y ubicación de las fugas, esta metodología no introducirá cambios en la topología de la red con lo que no afectará a la frecuencia de intervención. En cambio, sí que afectará al rendimiento de los trabajos de ubicación. Con 15 sensores de presión se obtiene una detección de la localización exacta en el $39,5 \%$ de la red, una detección aproximada en el $42,1 \%$ y en el $18,4 \%$ de las ubicaciones no se podrá detectar la localización. Por tanto, el sistema de búsqueda de fugas deberá funcionar a lo largo de 9,21 km de red. Para esta longitud, el tiempo de correlación es el mostrado en la Tabla 5.12. 
Tabla 5.12 Tiempo de correlación para la red sensorizada

\begin{tabular}{|c|c|c|}
\hline Red & $\begin{array}{c}\text { Tiempo de correlación } \\
\text { (jornadas) }\end{array}$ & $\begin{array}{c}\text { Velocidad de } \\
\text { inspección }(\mathbf{m} / \mathbf{h})\end{array}$ \\
\hline Red sensorizada & 3,84 & 1.629 \\
\hline
\end{tabular}

Con todo ello, el coste asociado a esta política de gestión de fugas bajo el uso de sensores de presión y caudal supondrá un total anual de $110.501 €$.

\subsubsection{Capacidad hidráulica de la red resultante}

Según la literatura existente y marcado como objetivo el detectar modificación alguna sobre la capacidad hidráulica de la red al implantar alguna de las dos técnicas planteadas, se han seleccionado una serie de indicadores para ello. Este apartado se ha centrado o bien en indicadores relacionados directamente con los niveles de presiones, por su dependencia con el nivel fugado, o bien en los indicadores relacionados con la robustez del sistema, por la capacidad misma de la red para afrontar escenarios críticos como puede ser el caso de un incendio.

Dentro de los niveles de presiones, se empezarán a analizar los datos de presión obtenidos directamente del modelo de simulación para el rango temporal analizado. Así, los valores más relevantes para los escenarios analizados se muestran en la Tabla 5.13:

Tabla 5.13 Niveles de presiones

\begin{tabular}{|c|c|c|c|}
\hline Indicador & Red inicial & $\begin{array}{c}\text { Red } \\
\text { Sectorizada }\end{array}$ & $\begin{array}{c}\text { Red } \\
\text { Sensorizada }\end{array}$ \\
\hline Presión media (mca) & 37,73 & 31,20 & 37,73 \\
\hline Presión máxima (mca) & 47,07 & 47,02 & 47,07 \\
\hline$P_{\max } / P_{\text {media }}(-)$ & 1,24 & 1,51 & 1,24 \\
\hline Presión mínima (mca) & 31,74 & 20,05 & 31,74 \\
\hline$P_{\text {min }} / P_{\text {media }}(-)$ & 0,84 & 0,64 & 0,84 \\
\hline$P_{\text {media }} / P_{\text {min admisible }}(-)$ & 1,89 & 1,56 & 1,89 \\
\hline
\end{tabular}

Siguiendo con el nivel de presiones, se van a emplear los indicadores propuestos por Di Nardo et al. (2013a; 2014) y Tzatchkov et al. (2014) y recogidos en el apartado 2.6.3: exceso de presión media, déficit de presión y desviación de la presión respecto a la presión de diseño. De esta menara, se obtienen los valores mostrados en la Tabla 5.14 para los diferentes escenarios.

Tabla 5.14 Exceso de presión media, déficit de presión y desviación de la presión

\begin{tabular}{|c|c|c|c|}
\hline Indicador & Red inicial & $\begin{array}{c}\text { Red } \\
\text { Sectorizada }\end{array}$ & $\begin{array}{c}\text { Red } \\
\text { Sensorizada }\end{array}$ \\
\hline Exceso de presión media (mca) & 17,62 & 9,40 & 17,62 \\
\hline Déficit de presión (mca) & 0 & 0 & 0 \\
\hline $\begin{array}{c}\text { Desviación de la presión respecto } \\
\text { a la presión de diseño (mca) }\end{array}$ & 18,16 & 13,92 & 18,16 \\
\hline
\end{tabular}


Este último indicador, desviación de la presión respecto a la presión de diseño, mide la dispersión de los datos de presión en los nudos respecto a su presión de diseño. Esta dispersión también puede obtenerse sobre la presión media de la red (Tabla 5.15) o sobre cualquier escenario fijado.

Tabla 5.15 Desviación sobre la presión media

\begin{tabular}{|c|c|c|c|}
\hline Indicador & Red inicial & $\begin{array}{c}\text { Red } \\
\text { Sectorizada }\end{array}$ & $\begin{array}{c}\text { Red } \\
\text { Sensorizada }\end{array}$ \\
\hline $\begin{array}{c}\text { Desviación de la presión respecto } \\
\text { a la presión media (mca) }\end{array}$ & 3,17 & 8,26 & 3,17 \\
\hline
\end{tabular}

Para terminar con el nivel de presiones, se calculará el índice HP (Coelho, 1997) para todos los escenarios (Tabla 5.16).

Tabla 5.16 Cálculo del indicador HP

\begin{tabular}{|c|c|c|c|}
\hline Indicador & Red inicial & $\begin{array}{c}\text { Red } \\
\text { Sectorizada }\end{array}$ & $\begin{array}{c}\text { Red } \\
\text { Sensorizada }\end{array}$ \\
\hline HP & 1,14 & 2,20 & 1,14 \\
\hline
\end{tabular}

Para el cálculo de este último indicador se han asignado los siguientes pesos para los diferentes niveles de presión: un valor de 3 para la presión adecuada de servicio, 2 para un nivel aceptable de servicio (más del $25 \%$ de la presión de servicio), 1 para una presión inadecuada (más del $75 \%$ de la presión de servicio) y 0 cuando no se consigue suministrar con la presión mínima de servicio (0.75 x presión mínima).

Tras analizar los niveles de presiones, el segundo aspecto a abordar se trata de la confiabilidad de las redes. El primer parámetro por estudiar es el comportamiento de las redes bajo condiciones de incendio, y para ello se recurrirá al indicador propuesto en el aparatado 2.6.6, cuyos cálculos se recogen en la Tabla 5.17.

Tabla 5.17 Cálculo del IPI

\begin{tabular}{|c|c|c|c|}
\hline Indicador & Red inicial & $\begin{array}{c}\text { Red } \\
\text { Sectorizada }\end{array}$ & $\begin{array}{c}\text { Red } \\
\text { Sensorizada }\end{array}$ \\
\hline IPI & 10 & 163 & 10 \\
\hline $\begin{array}{c}\text { No de escenarios de incendio } \\
\text { con incumplimientos }\end{array}$ & $\begin{array}{c}6 \\
15,79 \%\end{array}$ & $\begin{array}{c}38 \\
100 \%\end{array}$ & $\begin{array}{c}6 \\
15,79 \%\end{array}$ \\
\hline
\end{tabular}

Los siguientes cuatro indicadores han sido seleccionados por varios autores (apartado 2.5.1.). para el estudio de la energía disipada en la red. Se tratan de dos indicadores relacionados con la fiabilidad del sistema y la variación sufrida en los mismos tras introducir una modificación en las redes. Los valores de estos indicadores para los escenarios estudiados son:

Tabla 5.18 Cálculo de indicadores energéticos

\begin{tabular}{|c|c|c|c|}
\hline Indicador & Red inicial & $\begin{array}{c}\text { Red } \\
\text { Sectorizada }\end{array}$ & $\begin{array}{c}\text { Red } \\
\text { Sensorizada }\end{array}$ \\
\hline Índice de fiabilidad & 0,59 & 0,31 & 0,59 \\
\hline
\end{tabular}




\begin{tabular}{|c|c|c|c|}
\hline Desviación del índice de fiabilidad & - & $-47,46 \%$ & $0 \%$ \\
\hline Índice de la robustez de la red & 0,62 & 0,37 & 0,62 \\
\hline Desviación del índice de robustez & - & $-40,3 \%$ & $0 \%$ \\
\hline
\end{tabular}

Los últimos indicadores por calcular en este apartado corresponderán con la energía entrega a los usuarios, la energía disipada en la fricción en el transporte de esa agua y la eficiencia energética dinámica (Tabla 5.19).

Tabla 5.19 Cálculo de indicadores energéticos

\begin{tabular}{|c|c|c|c|}
\hline Indicador & Red inicial & $\begin{array}{c}\text { Red } \\
\text { Sectorizada }\end{array}$ & $\begin{array}{c}\text { Red } \\
\text { Sensorizada }\end{array}$ \\
\hline Energía entregada a los usuarios $(\mathrm{kWh} / \mathrm{h})$ & 28,05 & 21,92 & 28,05 \\
\hline Energía perdida por fricción en tuberías $(\mathrm{kWh} / \mathrm{h})$ & 9,23 & 15,36 & 9,23 \\
\hline Eficiencia energética dinámica $(\%)$ & 53,16 & 68,02 & 53,16 \\
\hline
\end{tabular}

\subsubsection{Calidad del agua de la red resultante}

Para la evaluar el efecto de la sectorización o sensorización de una red sobre el deterioro de la calidad del agua mientras circula por las tuberías, al igual que las recomendaciones recogidas en el apartado 2.6.4, se empleará el software EPANET para obtener el tiempo de permanencia del agua en la red antes y después de implantar dichas técnicas. En la Tabla 5.20 se recogen los tiempos de permanencia medios del agua en las tuberías.

Tabla 5.20 Tiempo de permanencia media del agua en tuberías

\begin{tabular}{|c|c|c|c|}
\hline Indicador & Red inicial & $\begin{array}{c}\text { Red } \\
\text { Sectorizada }\end{array}$ & $\begin{array}{c}\text { Red } \\
\text { Sensorizada }\end{array}$ \\
\hline Tiempo de permanencia media $(\mathrm{h})$ & 2,43 & 5,76 & 2,43 \\
\hline
\end{tabular}

Adicionalmente, y con el empleo del mismo software, se estudiará cómo se verá afectada la concentración de cloro en la red. Como punto de partida, y siguiendo lo marcado en el RD $902 / 2018$, en todos los puntos de la red deberá existir una concentración de cloro comprendida en el rango 0.4-1 ppm. Para dicha simulación se tomará un coeficiente de difusión relativo igual a 1 y el orden de Reacción en el medio y en la pared igual a -0.5. El coeficiente global de reacción en el medio se fijará en -0,1, estableciendo así un decrecimiento en el tiempo de la concentración de cloro, y se supondrá un mismo valor para todas las tuberías. Del mismo modo, para el coeficiente global de reacción en las tuberías se estimará un valor de -0,1, ya que al igual que con el tiempo se incrementará la rugosidad de las tuberías también se incrementará la reactividad del cloro con las paredes de las tuberías. Como en el caso anterior, se fijará un mismo valor para todas las tuberías. Y en último lugar, se fija una concentración mínima de cloro de 0 ppm. Así, las concentraciones medias y mínimas en los puntos de consumo se muestran en la Tabla 5.21, partiendo de una dosificación contante de 0,9 ppm en ambos depósitos. 
Tabla 5.21 Niveles de concentración de cloro en los puntos de consumo

\begin{tabular}{|c|c|c|c|}
\hline $\begin{array}{c}\text { Concentración de cloro en } \\
\text { punto de consumo }\end{array}$ & Red inicial & $\begin{array}{c}\text { Red } \\
\text { Sectorizada }\end{array}$ & $\begin{array}{c}\text { Red } \\
\text { Sensorizada }\end{array}$ \\
\hline Concentración media (ppm) & 0,73 & 0,73 & 0,73 \\
\hline Concentración mínima (ppm) & 0,48 & 0,35 & 0,48 \\
\hline
\end{tabular}

\subsubsection{Coste de implantación}

Como último objetivo, y no por ello menos importante, se contemplan los costes relativos a la implantación de las técnicas a estudiar. Dentro de este apartado se deberán diferenciar entre los costes de implantación directos, como aquellos costes necesarios para el desarrollo de la metodología seleccionada, y otros costes, que siendo costes que mejorarán el servicio no son obligatorios para la prestación de este.

Como costes de implantación directos, en la sectorización de la red se deberán contemplar la instalación de los contadores generales y de las válvulas de corte. En el caso de la sensorización de la red, dentro de este apartado se deberán contemplar la instalación de los sensores de presión y los caudalímetros necesarios. Al margen de los materiales y su instalación, también se deberán contemplar los equipos de comunicación necesarios para integrar las señales recibidas de presión y caudal en los SCADAS centrales.

En el caso de la sectorización de la red será necesario instalar 3 contadores sectoriales, 9 válvulas de corte y los correspondientes equipos de telegestión. Siendo así el coste total de implantación de $22.620 €$, según el detalle de la Tabla 5.22.

Tabla 5.22 Costes directos para sectorizar la red

\begin{tabular}{|l|c|c|c|}
\hline Equipos & Ud. & $\begin{array}{c}\text { Coste } \\
\text { unitario }(\boldsymbol{\epsilon})\end{array}$ & $\begin{array}{c}\text { Coste total } \\
(\boldsymbol{(})\end{array}$ \\
\hline Contadores sectoriales sobre tubería DN200 & 2 & 3.750 & 7.500 \\
\hline Contadores sectoriales sobre tubería DN90 & 1 & 2.150 & 2.150 \\
\hline Válvulas de corte DN200 & 1 & 1.750 & 1.750 \\
\hline Válvulas de corte DN150 & 1 & 1.600 & 1.600 \\
\hline Válvulas de corte DN50 & 1 & 600 & 600 \\
\hline Válvulas de corte DN80 & 4 & 730 & 2.920 \\
\hline Válvulas de corte DN100 & 2 & 1.100 & 2.200 \\
\hline Equipos de comunicación GPRS (Q+P) & 3 & 1.300 & 3.900 \\
\hline TOTAL & & & $\mathbf{2 2 . 6 2 0}$ \\
\hline
\end{tabular}

En el caso de sensorizar la red se optó por instalar 15 sensores de presión, ningún caudalímetro y los correspondientes equipos de telegestión. En este caso, el coste total de implantación necesario asciende a 33.000€ (Tabla 5.23). 
Tabla 5.23 Costes directos para sensorizar

\begin{tabular}{|l|c|c|c|}
\hline Equipos & Ud. & $\begin{array}{c}\text { Coste } \\
\text { unitario }(\boldsymbol{\epsilon})\end{array}$ & $\begin{array}{c}\text { Coste total } \\
(\boldsymbol{\epsilon})\end{array}$ \\
\hline Sensor de presión & 15 & 1.100 & 16.500 \\
\hline Equipos de comunicación GPRS (P) & 15 & 1.100 & 16.500 \\
\hline TOTAL & & & 33.000 \\
\hline
\end{tabular}

No se consideran, para ninguna de las dos alternativas, otros costes. Un ejemplo de estos podría ser el mallado de la red a sectorizar para conseguir mejores respuestas hidráulicas de la red.

Dentro del este apartado, que correspondería con un análisis económico de la inversión se debería contemplar tanto el mantenimiento de las futuras infraestructuras como una evaluación de la rentabilidad misma del proyecto. Pero debido a que el objetivo del presente estudio comparativo entre técnicas es otro, no se tendrá en cuenta este estudio de viabilidad económica de las mismas.

\subsubsection{Valoración de las alternativas}

Una vez analizadas todas las métricas, se deberán transformar dichos valores absolutos en valores relativos siguiendo los rangos establecidos en la Tabla 5.4. En la Tabla 5.24 se muestran dichos valores.

Tabla 5.24 Métricas de evaluación y valores de referencia

\begin{tabular}{|c|c|c|c|c|c|c|}
\hline \multirow{2}{*}{ Objetivos } & \multirow{2}{*}{$\begin{array}{c}\text { Criterios } \\
\text { de } \\
\text { evaluación }\end{array}$} & \multirow{2}{*}{ Métricas } & \multicolumn{2}{|c|}{ Sectorización } & \multicolumn{2}{|c|}{ Sensorización } \\
\hline & & & $\begin{array}{c}\text { Valor } \\
\text { absoluto }\end{array}$ & $\begin{array}{l}\text { Valor } \\
\text { relativo }\end{array}$ & $\begin{array}{c}\text { Valor } \\
\text { absoluto }\end{array}$ & $\begin{array}{l}\text { Valor } \\
\text { relativo }\end{array}$ \\
\hline \multirow{2}{*}{ Objetivo 1} & Criterio 1.1 & Reducción en el coste anual & $42 \%$ & 6,00 & $52 \%$ & 6,8 \\
\hline & Criterio 1.2 & Velocidad de inspección & 1398 & 7,99 & 1629 & 8,76 \\
\hline \multirow{10}{*}{ Objetivo 2} & \multirow{4}{*}{ Criterio 2.1} & $\begin{array}{l}\text { Variabilidad de la presión } \\
\text { media }\end{array}$ & 8,26 & 8,62 & 3,17 & 9,47 \\
\hline & & Exceso de presión media & 9,4 & 6,87 & 17,62 & 4,13 \\
\hline & & Déficit de presión media & 0 & 10 & 0 & 10 \\
\hline & & HP & 2,2 & 7,33 & 1,14 & 3,8 \\
\hline & \multirow{3}{*}{ Criterio 2.2} & IPI & 100 & 0 & 15,95 & 1,35 \\
\hline & & Índice de fiabilidad & 0,31 & 3,13 & 0,59 & 5,96 \\
\hline & & Índice de la robustez & 0,37 & 3,74 & 0,62 & 6,26 \\
\hline & \multirow{3}{*}{ Criterio 2.3} & $\begin{array}{c}\text { Exceso de energía entregada } \\
\text { a los usuarios }\end{array}$ & $47 \%$ & 7,76 & $88 \%$ & 5,91 \\
\hline & & & & & & \\
\hline & & Eficiencia energética dinámica & 68,02 & 7,87 & 53,16 & 6,88 \\
\hline \multirow{4}{*}{ Objetivo 3} & \multirow{2}{*}{ Criterio 3.1} & $\begin{array}{l}\text { Tiempo máximo de } \\
\text { permanencia }\end{array}$ & 24 & 0 & 12,55 & 4,77 \\
\hline & & Tiempo medio de permanencia & 4,83 & 7,99 & 2,43 & 8,99 \\
\hline & \multirow{2}{*}{ Criterio 3.3} & Concentración media de cloro & 0,73 & 7 & 0,73 & 7 \\
\hline & & $\begin{array}{c}\text { Concentración mínima de } \\
\text { cloro }\end{array}$ & 0,35 & 2,92 & 0,48 & 4,22 \\
\hline
\end{tabular}




\begin{tabular}{|c|c|c|c|c|c|c|}
\hline \multirow{2}{*}{ Objetivos } & $\begin{array}{c}\text { Criterios } \\
\text { de } \\
\text { evaluación }\end{array}$ & Métricas & \multicolumn{2}{|c|}{ Sectorización } & \multicolumn{2}{c|}{ Sensorización } \\
\cline { 3 - 7 } & Criterio 4.1 & $\begin{array}{c}\text { Costes de implantación } \\
\text { directos }\end{array}$ & $\begin{array}{c}\text { Valor } \\
\text { absoluto }\end{array}$ & $\begin{array}{c}\text { Valor } \\
\text { relativo }\end{array}$ & $\begin{array}{c}\text { Valor } \\
\text { absoluto }\end{array}$ \\
\hline \multirow{2}{*}{ Objetivo 4} & Crivo \\
\cline { 2 - 7 } & Criterio 4.2 & Otros costes & 0 & 10 & 0 & 8,53 \\
\hline
\end{tabular}

Multiplicando dichos valores relativos por su peso asociado (Tabla 5.3) se obtendrá la valoración de cada uno de los criterios, objetivos y la evaluación final entre las dos técnicas objeto de este estudio (Tabla 5.26).

Tabla 5.25 Evaluación de los objetivos y criterios

\begin{tabular}{|c|c|c|c|c|c|}
\hline \multirow{4}{*}{$\begin{array}{l}\text { Objetivos } \\
\text { Objetivo } 1\end{array}$} & \multirow{3}{*}{$\begin{array}{l}\text { Criterios de } \\
\text { evaluación }\end{array}$} & \multicolumn{4}{|c|}{ Valoración } \\
\hline & & \multicolumn{2}{|c|}{ Sectorización } & \multicolumn{2}{|c|}{ Sensorización } \\
\hline & & 6,00 & 605 & 6,80 & \\
\hline & Criterio 1.2 & 7,99 & & 8,76 & דט, \\
\hline \multirow{3}{*}{ Objetivo 2} & Criterio 2.1 & 8,28 & \multirow{3}{*}{6,25} & 6,92 & \multirow{3}{*}{6,12} \\
\hline & Criterio 2.2 & 2,50 & & 4,81 & \\
\hline & Criterio 2.3 & 7,82 & & 6,46 & \\
\hline \multirow{2}{*}{ Objetivo 3} & Criterio 3.1 & 2,66 & \multirow{2}{*}{3,92} & 6,18 & \multirow{2}{*}{5,61} \\
\hline & Criterio 3.3 & 4,55 & & 5,33 & \\
\hline \multirow{2}{*}{ Objetivo 4} & Criterio 4.1 & 8,99 & \multirow{2}{*}{9,33} & 8,53 & \multirow{2}{*}{9,02} \\
\hline & Criterio 4.2 & 10,00 & & 10,00 & \\
\hline
\end{tabular}

Tabla 5.26 Evaluación final entre las dos técnicas

\begin{tabular}{|c|c|}
\hline Técnica & Valoración Final \\
\hline Sectorización & 6,38 \\
\hline Sensorización & 6,98 \\
\hline
\end{tabular}

\subsubsection{Discusión de los resultados obtenidos}

En el primer caso de estudio, la sectorización del sistema influye positivamente sobre los trabajos de búsqueda de fugas. El mero hecho de dividir la red en subsistemas independientes hace que la política de intervención se aplique a cada sector de manera aislada y sin influencia del resto. La frecuencia de intervención está marcada por la frecuencia de aparición de las fugas, la cual, a su vez, depende de los kilómetros de red. Es por ello qué, al dividir la red, los periodos de intervención de cada sector aumenten, al disminuir los kilómetros englobados en cada sector. Este hecho obligará a mayores volúmenes perdidos de agua, pero a una importante reducción de los costes de inspección. En total, la política de gestión de fugas seguida aplicada en la sectorización planteada hará que los costes de intervención e inspección y los asociados al agua perdida se vean reducidos en un $42 \%$. En el caso de la segunda técnica, la sensorización no modificará la frecuencia de intervención, pero sí reducirá 
la longitud a revisar en cada intervención. Ello hará que los costes anuales se reduzcan en un $52 \%$. Cabe destacar que estos resultados no se pueden extrapolar a todas las estratégicas de intervención, sino que de los resultados obtenidos dependerán del tipo de política de intervención seguida. Otro aspecto importante que destacar es el importante aumento sufrido en la velocidad de inspección de la red. Inicialmente, el rendimiento de los trabajos de correlación y escucha era de 300 metros revisados a la hora. En el caso de la sectorización, al detectar en qué sector se produce la fuga la longitud a revisar disminuyó, o lo que es lo mismo, mucha longitud se puede suponer como inspeccionada. Se pasa, así, a un rendimiento de 1.398 metros a la hora. En el caso de la sensorización, esta velocidad de inspección es aún mayor, alcanzando los 1.629 metros a la hora.

Desde el punto de vista de la capacidad hidráulica de la red, se obtienen grandes diferencias entre ambos escenarios, como era de esperar. La sectorización es una técnica intrusiva, que obliga el cierre de ciertas tuberías para formar los sectores, cambiando para ello la topología de la red. En el caso de estudio, al sectorizar se está aumentando la energía perdida por fricción en un 66,4\% y, con ello, disminuyendo los niveles de presiones en la red. Se pasa así de una presión media de 44,55 mca a 37,11 mca, un 16,7\% menos. Respecto a los niveles extremos, sí se ha reducido la presión mínima, quedando la presión máxima en los mismos niveles. Esta disminución de los niveles de presión se observa de manera generalizada en el resto de los indicadores calculados. Por ejemplo, el exceso de la presión media se ha reducido en un $46 \%$. Aunque el nivel medio de presión se ha visto reducido, no lo ha hecho la desviación de la presión con respecto a su valor medio. Respecto a la distribución de los valores de presión, el escenario sin sectorizar presenta una desviación de 3,17 mca. En cambio, en la red sectorizada se aumenta esta desviación hasta los 8,26 mca. Esto es debido a la estratificación impuesta por la definición de los sectores. Pero aun presentando mayores desviaciones, la red sectorizada presenta un nivel general de las presiones con un valor de HP de 2,20, frente a los 1,14 que presenta la red sin sectorizar. Es decir, unos valores más adecuados de presiones en la red. Abordando la resiliencia de ambos escenarios, se obtienen unos índices de fiabilidad similares, con lo que la redundancia energética de ambas redes es la misma. En cambio, y debido al cierre de mallas, en la sectorización de la red se obtiene una disminución del índice de robustez de un 40,3\%. Por lo que, la red inicial sin alterar presenta unos valores más altos de exceso de energía disponible, una distribución de tuberías más uniformes y, por lo tanto, mayores capacidades de recuperación de la red. Este hecho se puede apreciar a la hora de analizar el comportamiento de la red ante condiciones de incendio. En el caso de las sectorización, y debido al cierre de tuberías, en todos los escenarios posibles la red no es capaz de suministrar en las condiciones necesarias, frente al $15,79 \%$ de las veces que ocurre cuando se encuentran todas las tuberías abiertas.

Desde el punto de vista de la calidad del agua, los tiempos de permanencia del agua en la red aumentan al sectorizar la red, pasando de 2,43 horas a 5,76 horas. Para la red sensorizada, no se producen cambios sobre la red, por lo tanto, estos valores de calidad no se modifican respecto al escenario inicial. Los niveles de concentración medios y mínimos de cloro en red se mantienen prácticamente iguales para ambos escenarios, aunque ligeramente superiores para el caso de la sectorización.

El último punto analizado ha sido el punto económico. Ambas técnicas requieren de ciertas inversiones para poder ejecutarse. En el caso de la sectorización, es necesaria la instalación de contares y válvulas de corte. Y la vía de la sensorica, igualmente, requiere de sensores de presión o caudal y los correspondientes equipos de comunicación. Aun así, no se puede establecer un criterio general sobre el coste de cada una de las técnicas, ya que el 
presupuesto final dependerá de la situación particular de cada caso. En el caso analizado, es necesario un coste ligeramente superior en el caso de la sensorización de la red.

Una vez obtenidas todas las métricas, se puede realizar la comparativa de todas ellas y conocer cuál de las dos técnicas desprende unos mejores resultados al sistema. En este caso, la sensorización presenta una valoración final ligeramente superior que la sectorización de esta bajo los criterios de evaluación marcados. Desglosando la evaluación final, se pueden observar unos resultados en línea con lo esperado a priori. Respecto al primer objetivo, efectividad y eficiencia en la búsqueda de fugas no declaradas, la sensorización de la red presenta unos mejores resultados debido a la reducción de los costes anuales vinculados al agua perdida y a las campañas de búsqueda y al aumento de la velocidad de inspección, simplemente por la reducción de la longitud a revisar. En el objetivo 2, capacidad hidráulica de la red resultante, las valoraciones han sido muy similares. Por un lado, el sectorizar la red ha mejorado el nivel de presiones y, con ello, la eficiencia energética dinámica. Pero por otro, se ha visto reducida la robustez del sistema. Desde el punto de vista de la calidad del agua, objetivo 3, sectorizar la red afecta negativamente a los parámetros analizados, haciendo que el agua permanezca más tiempo en la red y que, con ello, el nivel de cloro se vea mermado. Para terminar, el último objetivo que analiza el impacto económico de implantación de cada una de las metodologías es menor en el caso de la sensorización. 


\subsubsection{Caso práctico 2. Red de distribución real}

\subsubsection{Planteamiento del caso}

El segundo caso de estudio consiste en un sistema urbano real de distribución de agua potable cuyo objeto es abastecer a una población de más de 4.600 habitantes. Para ello, cuenta con una longitud total de tuberías de $30,8 \mathrm{~km}$, con diámetros comprendidos entre los 30 y $150 \mathrm{~mm}$. Alrededor de $810 \mathrm{~m}^{3}$ de agua se entregan diariamente al sistema, de los cuales $540 \mathrm{~m}^{3}$ son consumidos. Se asume que el resto del volumen se pierde en el transporte, lo que deja un rendimiento hidráulico medio del $66,7 \%$.

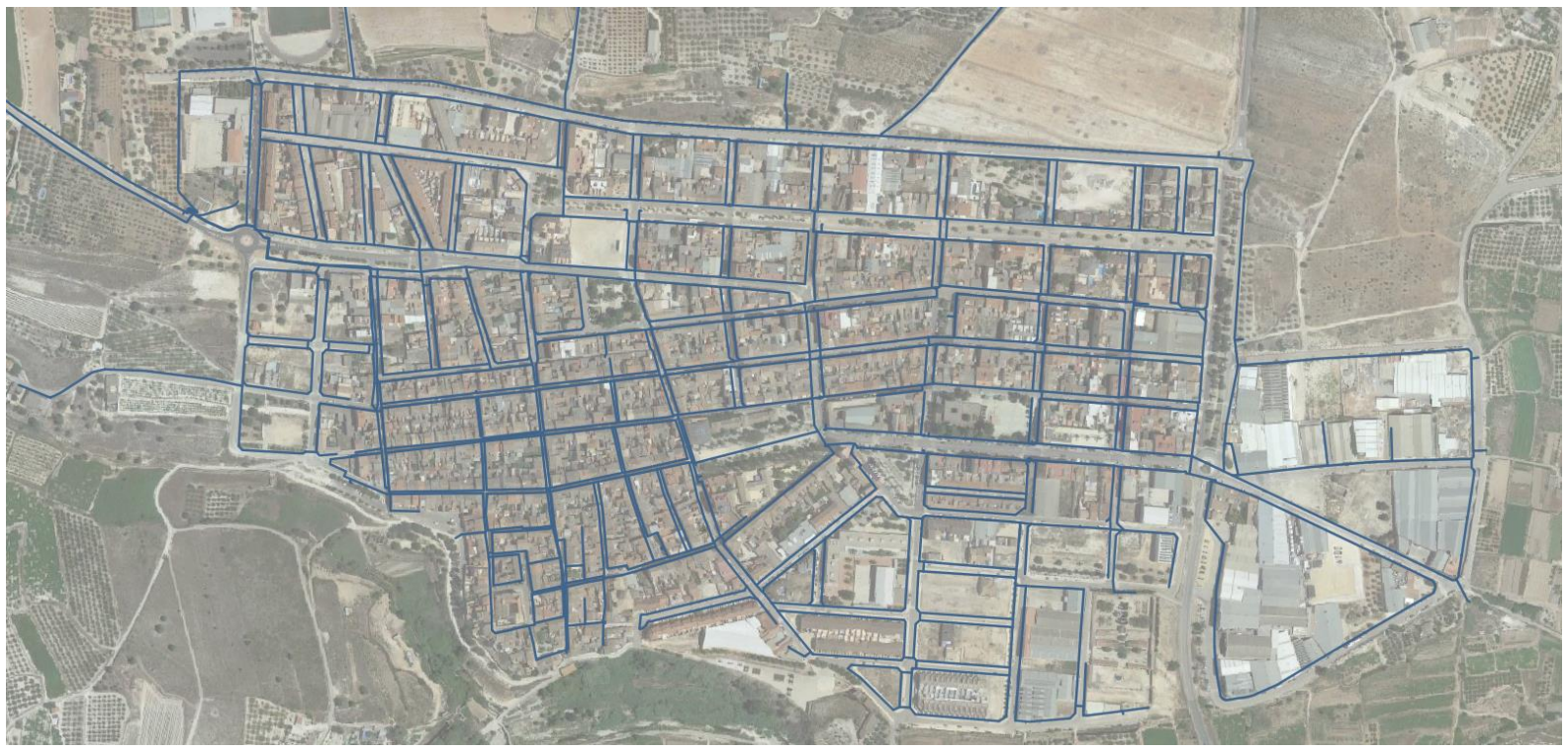

Figura 5.16 Red de distribución del caso 2

Esta red se encuentra dividida en dos sectores hidráulicos independientes, los cuales fueron diseñados siguiendo procesos prácticos y procedimientos empíricos. Las características de ambos sectores se recogen en la Tabla 5.27. El sector Zona Alta, sombreado en azul en la Figura 5.17, engloba el 60,7\% de la longitud de la red y un total de 991 acometidas. El segundo sector, Zona Baja, que se encuentra sombreado en verde, está compuesto por el 39,3\% de las tuberías y 801 acometidas. Además, este sector cuenta con una válvula reductora de presión a su entrada para reducir el nivel medio de presiones. Respecto al reparto del volumen suministrado, el sector Zona Alta demanda el 70,7\% del volumen total entregado al sistema empleando para ello una tubería de PE de $110 \mathrm{~mm}$, siendo el 29,3\% restante enviado al sector Zona Baja por medio de una tubería de FC de $150 \mathrm{~mm}$. Todo el volumen consumido es registrado, repartido en un $57 \%$ en la Zona Alta y el $43 \%$ restante en el sector Zona Baja.

Tabla 5.27 Características descriptivas de los sectores actuales

\begin{tabular}{|c|c|c|c|c|c|c|c|c|c|}
\hline \multirow{2}{*}{ Sector } & \multicolumn{2}{|c|}{ Longitud } & \multirow{2}{*}{$\begin{array}{c}\text { Diámetro } \\
\text { de } \\
\text { suministro }\end{array}$} & \multirow{2}{*}{$\begin{array}{c}\text { Gestión } \\
\text { de presión }\end{array}$} & \multicolumn{2}{|c|}{$\begin{array}{c}\text { Volumen } \\
\text { suministrado }\end{array}$} & \multicolumn{2}{|c|}{$\begin{array}{l}\text { Volumen } \\
\text { registrado }\end{array}$} & \multirow{2}{*}{$\begin{array}{c}\text { № de } \\
\text { acometidas }\end{array}$} \\
\hline & $(\mathrm{km})$ & (\%) & & & $\mathrm{m}^{3} /$ día & $(\%)$ & $\mathrm{m}^{3} /$ día & (\%) & \\
\hline Zona Alta & 18,7 & 60,7 & 110 & No & 573 & 70,7 & 308 & 57 & 991 \\
\hline Zona Baja & 12,1 & 39,3 & 150 & Sí & 237 & 29,3 & 232 & 43 & 801 \\
\hline
\end{tabular}




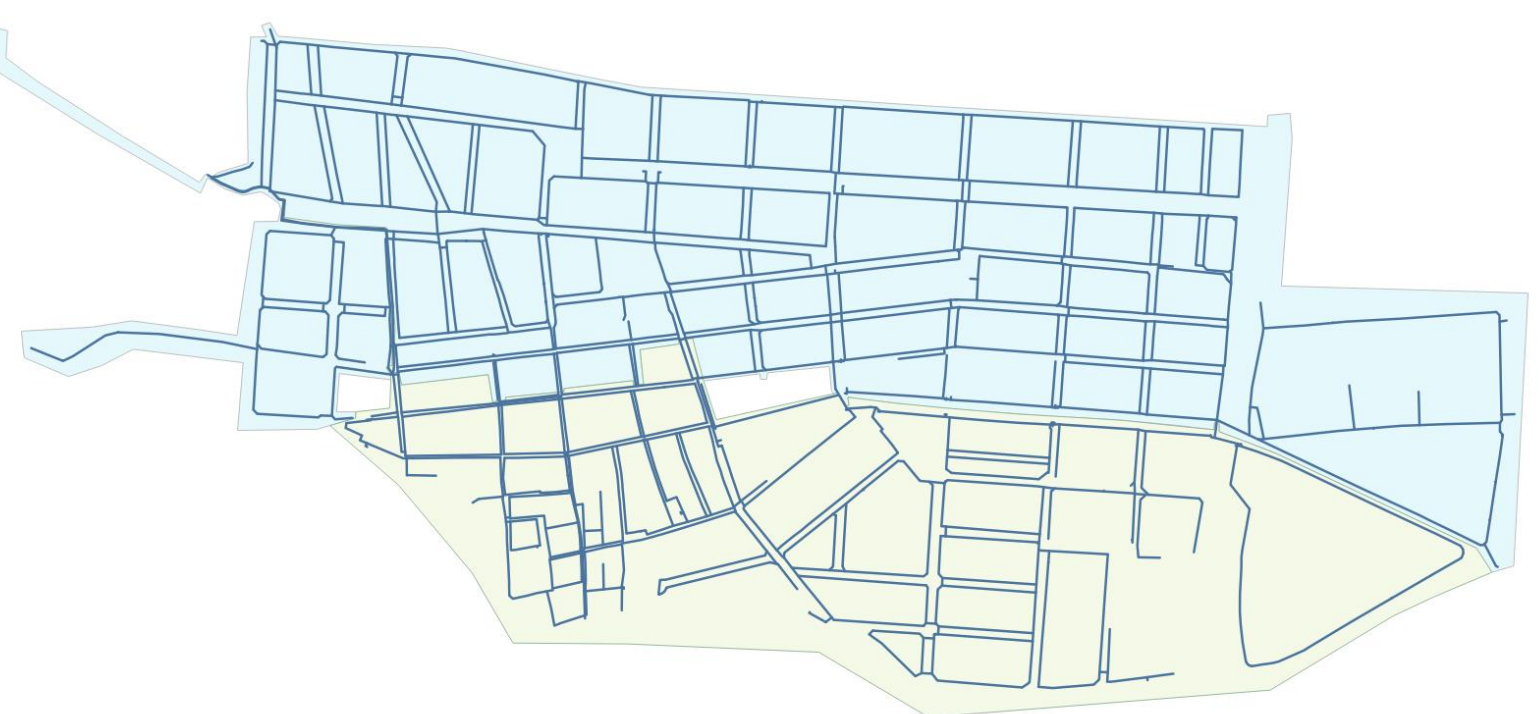

Figura 5.17 Distribución de la sectorización actual

El sistema cuenta con caudalímetro tanto a la salida del depósito de cabecera como a la entrada de los dos sectores, cuyos registros se reciben de manera continua. Todos los puntos de consumo están equipados con contador, y cuentan con un sistema de telelectura de red fija, el cual proporciona las lecturas horarias de todos ellos.

El comportamiento hidráulico de la red de este municipio se obtiene con la ayuda de un modelo de simulación, elaborado en Epanet (Rossman, 2000), y que incluye las fugas existentes en la misma, uniformemente distribuidas y agrupadas en los nudos de manera proporcional a la longitud de las tuberías (Almandoz et al., 2005). En su estado operativo normal, la presión promedio es de 44,6 mca, con valores extremos de 26,7 y 75,1 mca (Figura 5.20), frente a una presión mínima de servicio de $25 \mathrm{mca}$. La velocidad promedio del agua y la pérdida de carga unitaria en las tuberías son de $0,06 \mathrm{~m} / \mathrm{s}$ y $0,24 \mathrm{mca} / \mathrm{km}$, respectivamente (Figura 5.19).

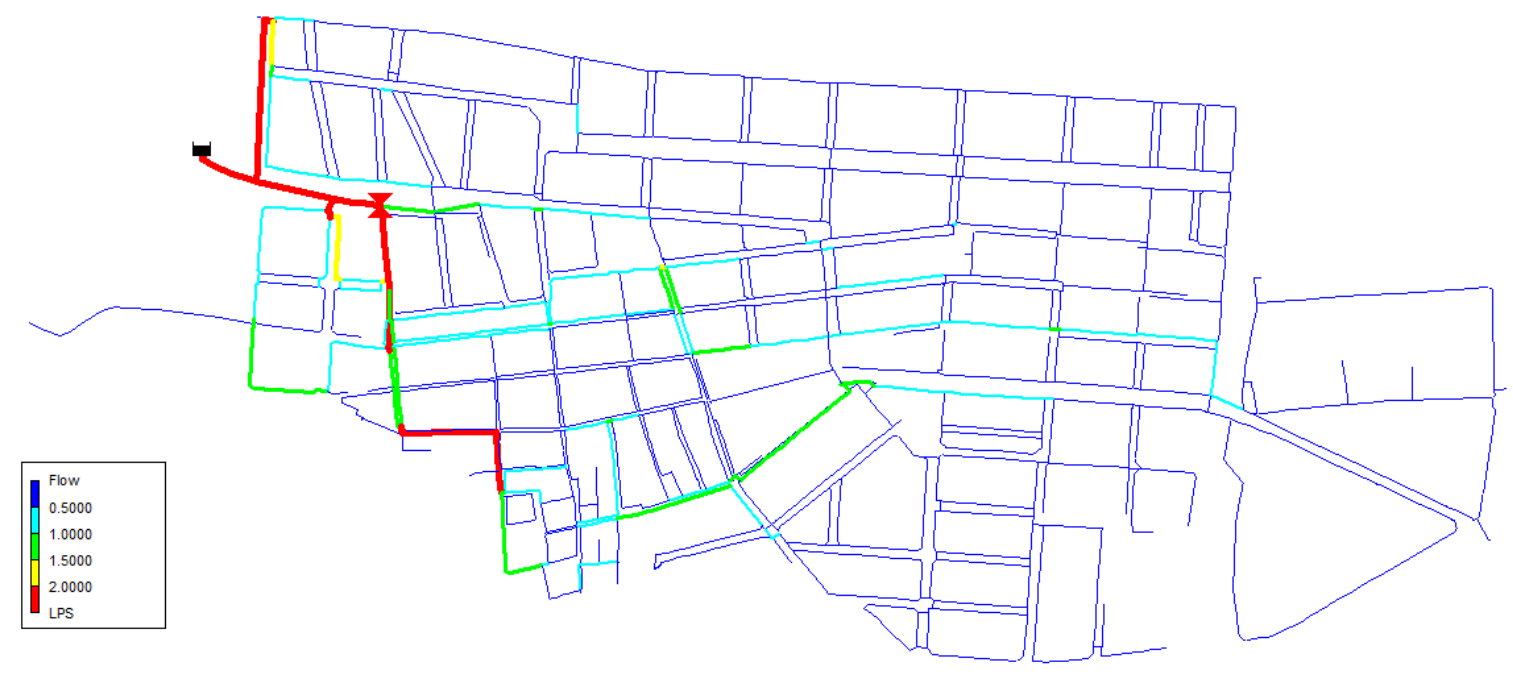

Figura 5.18 Caudales en las tuberías de distribución 


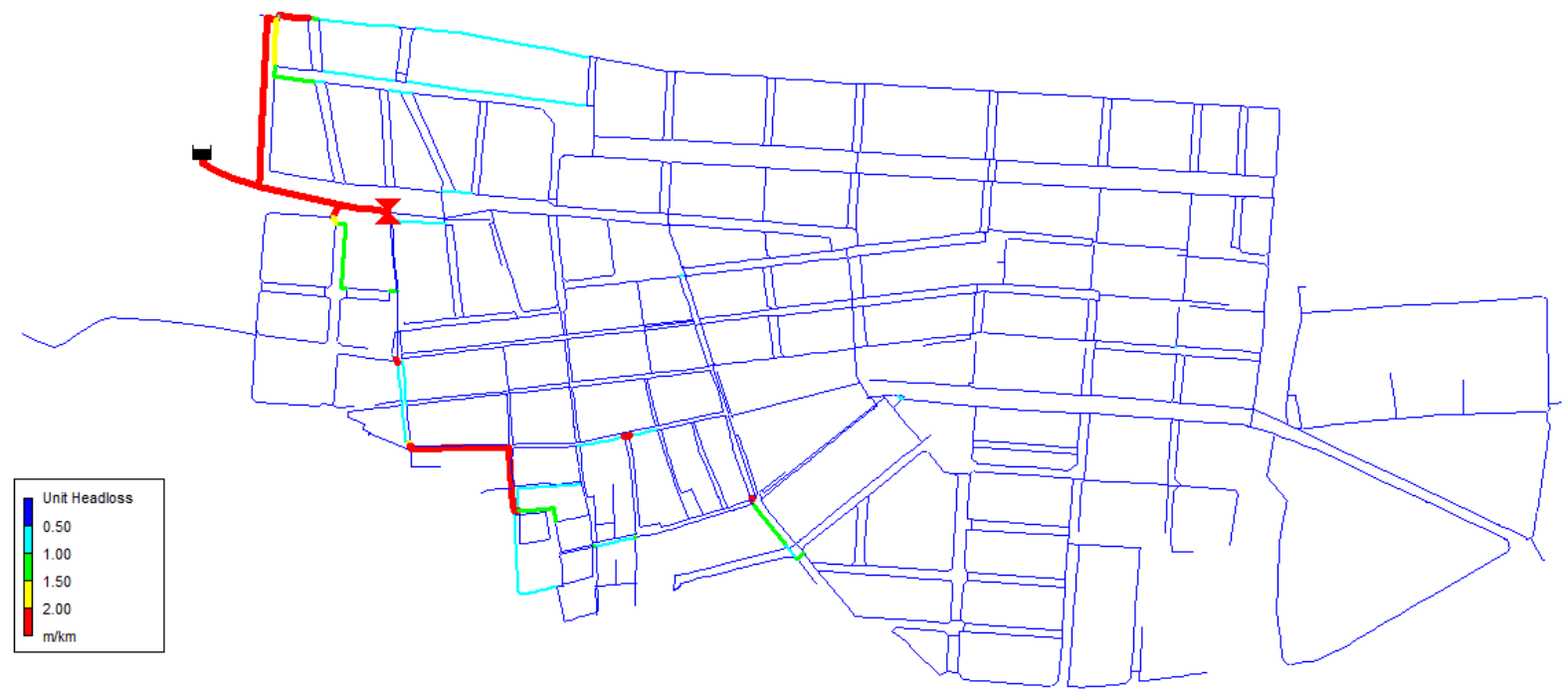

Figura 5.19 Pérdidas de carga en las tuberías de distribución

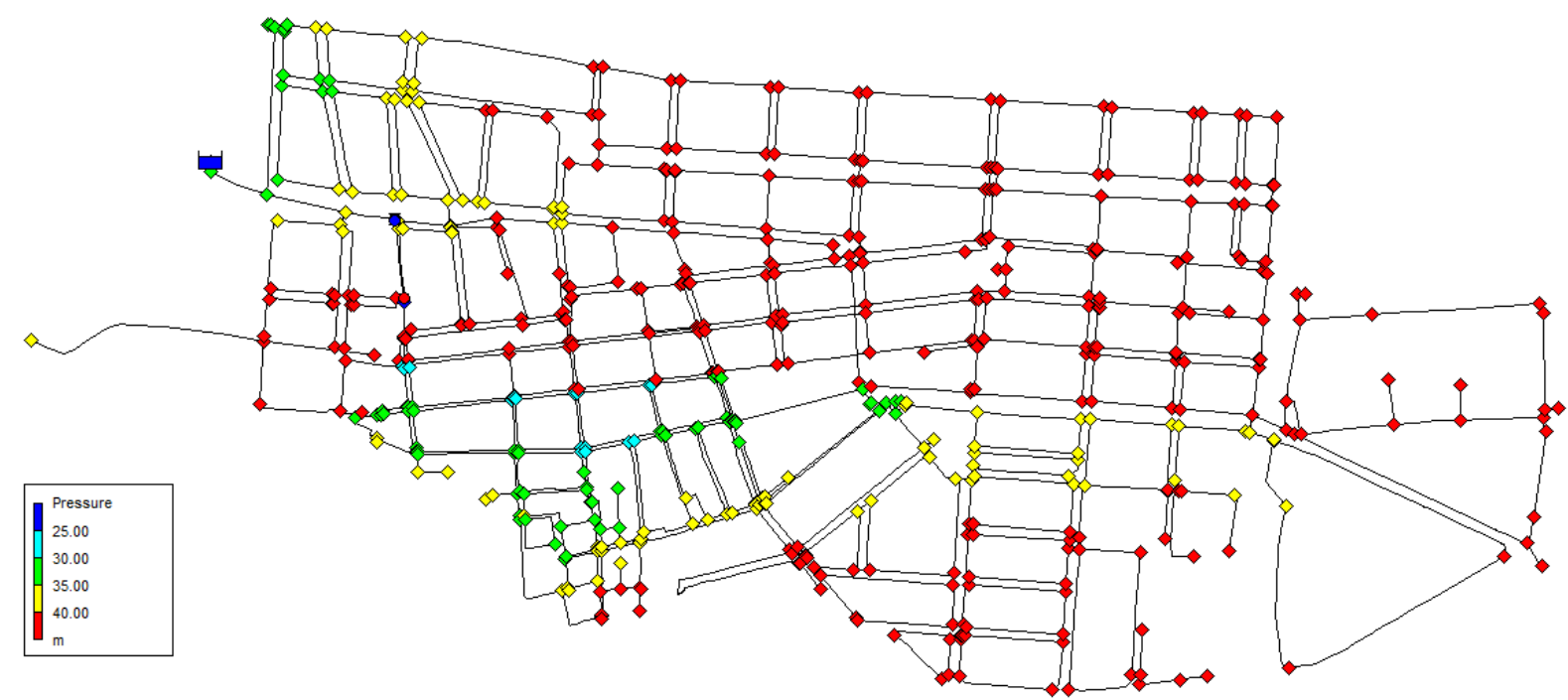

Figura 5.20 Nivel de presiones en los nudos

En cuanto al comportamiento de cada uno de los sectores, los resultados son diferentes (Tabla 5.28). El sector Zona Alta se alimenta directamente del depósito de cabecera sin el uso de válvulas de regulación, presentando una presión media de 49,48 mca en su estado operativo normal, con valores extremos de 32,30 y 75,12 mca. En el suministro del sector de la Zona Baja sí que se emplea una válvula reductora de presión para regular el nivel de estos, teniendo así un valor promedio de 37,49 mca, con valores extremos de 26,35 y 50,10 mca. Los niveles de fuga se estiman en 139,95 $\mathrm{l} / \mathrm{min}$ en el sector Zona Alta y 47,46 $\mathrm{l} / \mathrm{min}$ en la Zona Baja, frente a unos niveles mínimos de 99,27 y 38,40 l/min, respectivamente, suponiendo una presión mínima de servicio en cada nudo.

Tabla 5.28 Balance hídrico de la red y de cada uno de los sectores

\begin{tabular}{|l|c|c|c|}
\hline & $\begin{array}{c}\text { Sistema } \\
\text { Completo }\end{array}$ & $\begin{array}{c}\text { Sector Zona } \\
\text { Alta }\end{array}$ & $\begin{array}{c}\text { Sector Zona } \\
\text { Baja }\end{array}$ \\
\hline Caudal suministrado $\left(\mathrm{m}^{3} / \mathrm{d}\right)$ & 812,88 & 574,01 & 238,87 \\
\hline Caudal fugado $\left(\mathrm{m}^{3} / \mathrm{d}\right)$ & 269,87 & 201,53 & 68,34 \\
\hline
\end{tabular}




\begin{tabular}{|l|c|c|c|}
\hline Presión media (mca) & 44,55 & 49,48 & 37,49 \\
\hline Rango de presiones (mca) & $26,35-75,12$ & $32,30-75,12$ & $26,35-50,10$ \\
\hline Caudal fugado mínimo $\left(\mathrm{m}^{3} / \mathrm{d}\right)$ & 198,24 & 142,95 & 55,30 \\
\hline
\end{tabular}

Analizando energéticamente la red, bajo un escenario de comportamiento normal, se cuenta con una energía promedio introducida en el sistema de 124,32 kWh/día (Tabla 5.29). De los cuales el 83,8\% se entrega en forma de presión en los puntos de consumo y en las fugas, el $11,9 \%$ se localiza en el control y regulación, en este caso la válvula reductora de presión instalada a la entrada del sector de la Zona Baja, y el 4,2\% restante se disipa durante el transporte debido a la fricción del agua con las tuberías. Todo ello, dota al sistema de una eficiencia energética dinámica del 53,21\%. Entrando en detalle en cada uno de los sectores, se puede comprobar como el sector de la Zona Alta presenta unos valores de energía entregada en el grifo de consumidor mayores, principalmente por ser más elevados los valores de la presión. En la Zona Baja, parte de la energía introducida en el sistema se disipa en la válvula reductora de presión. Esto hace que el valor de la eficiencia energética dinámica sea mayor, 64,67\%. Otro aspecto a destacar es el bajo valor que presenta la energía disipada por fricción, lo cual va en concordancia con los bajos niveles de velocidad de circulación del agua.

Tabla 5.29 Balance energético de la red con fugas tras la sectorización según criterios energéticos

\begin{tabular}{|c|c|c|c|c|c|c|c|c|}
\hline \multirow[b]{2}{*}{ RED } & \multirow{2}{*}{$\begin{array}{c}\text { Demanda } \\
\left(\mathrm{m}^{3} / \mathrm{d}\right)\end{array}$} & \multicolumn{4}{|c|}{ TOTA ENERGÍA PERDIDA (kWh/d) } & \multirow{2}{*}{$\begin{array}{c}\text { Energía por } \\
\text { caudal } \\
\text { unitario } \\
\left(\mathrm{kWh} / \mathrm{m}^{3}\right)\end{array}$} & \multirow{2}{*}{$\begin{array}{l}\text { Mín. } \\
\text { energía en } \\
\text { grifo de } \\
\text { consumidor }\end{array}$} & \multirow{2}{*}{$\begin{array}{c}\text { Ef. } \\
\text { Energética } \\
\text { dinámica } \\
(\%)\end{array}$} \\
\hline & & Fricción & Control & $\begin{array}{c}\text { Grifo } \\
\text { consumidor }\end{array}$ & Total & & & \\
\hline $\begin{array}{l}\text { Sistema } \\
\text { completo }\end{array}$ & 818,88 & 5,28 & 14,88 & 104,16 & 124,32 & 0,15 & 55,44 & 53,21 \\
\hline $\begin{array}{c}\text { Sector } \\
\text { Zona Baja }\end{array}$ & 238,87 & 0,48 & 14,88 & 25,2 & 25,68 & 0,11 & 16,32 & 64,67 \\
\hline $\begin{array}{c}\text { Sector } \\
\text { Zona Alta }\end{array}$ & 574,01 & 4,8 & - & 78,96 & 83,76 & 0,15 & 39,12 & 49,57 \\
\hline
\end{tabular}

Desde un punto de vista operativo, se sigue una estrategia de gestión de fugas basada en el método del análisis de los caudales nocturnos. El nivel de fugas se monitorea continuamente y se toma acción tan pronto como se detecta un aumento del nivel nocturno superior a 250 $\mathrm{I} / \mathrm{min}$. La frecuencia de fugas sobre la red de distribución es de 0,46 fugas/(km·año), donde el $40 \%$ de las fugas son no declaradas, y el tamaño medio de cada fuga está en $60 \mathrm{l} / \mathrm{min}$. Respecto a las fugas en acometidas, se cuenta con una frecuencia media de 0,81 fugas/(km·año). Al igual que en el caso anterior, el $70 \%$ de las fugas en acometidas son declaradas y el tamaño medio se estima en $30 \mathrm{l} / \mathrm{min}$. Se asume que se tarda en ubicar y reparar las fugas no declaradas en red un tiempo medio de 3 días, siendo de 14 días para las fugas en acometidas. Respecto al caso de las declaradas, estos tiempos descienden a 1 y 7 días, respectivamente.

El coste medio del agua es de $0,11 € / \mathrm{m}^{3}$. Respecto a los costes de reparación de las fugas, se asumen independientes de la estrategia de gestión de fugas seguida, y por lo tanto no se tendrá presente para los análisis posteriores. Para cada sector, el coste anual de equipos y mantenimiento es de 1.087,80 €/año, según el siguiente detalle: un coste inicial de $18.000 €$ por sector (independientemente del tamaño de este), una vida útil de todos los equipos de 10 años, un coste anual de mantenimiento asumido en un $20 \%$ sobre el coste inicial y una tasa de descuento del $5 \%$. 
El equipo de correlación y escucha supondrá un coste anual de 158,57€ / km red y año, según el siguiente destalle: coste inicial de $39.000 €$ por equipo de trabajo, una vida útil de todos los equipos de 5 años, el coste de mantenimiento se asume en un $20 \%$ sobre el coste inicial y una tasa de descuento del $5 \%$.

Cada equipo de trabajo estará formado por un solo operario con un salario anual de $40.000 €$ de coste de empresa más un $50 \%$ en concepto de gastos generales. El rendimiento de trabajo de correlación y escucha será de 300 metros revisados a la hora, con jornadas de 8 horas.

\subsubsection{Sectorización según conceptos energéticos}

Una vez conocida la red de partida y cómo se encuentra sectorizada, a continuación, se planteará una sectorización alternativa a la misma pero basada únicamente en criterios energéticos y sin condiciones externas que limiten el diseño de los sectores.

Para ello, se partirá de la red actual (Figura 5.16) sin sectorizar y sin regulación de presiones, es decir, la red original, y se agruparán todos los nudos de consumo en $n$ grupos, de tal forma que la diferencia entre el valor de la eficiencia energética dinámica de los nudos del mismo grupo sea menor que con el resto de los nudos de otros grupos. Se busca así obtener grupos de nudos con eficiencias energéticas dinámicas similares, cuya disposición permita maximizar los efectos derivados de la creación de los sectores o posteriores reducciones de presiones. Como se ha citado al principio, no se impondrán restricciones externas que condicionen la creación de los sectores, aunque en este caso y para evitar un número elevado de sectores, económicamente inviable, se fija una longitud mínima de tuberías por sector del $15 \%$ de la longitud total de la red.

Como resultado de la agrupación de todos los elementos en diferentes grupos, de tal forma que se englobe un mínimo del $15 \%$ de la longitud total de tuberías y la eficiencia energética dinámica sea la mínima posible en cada uno de ellos, se consigue agrupar al $75,71 \%$ de las tuberías y al $91,03 \%$ de los nudos en un total de cinco zonas claramente diferenciadas (Figura 5.21). El resto de los elementos (grafiados en gris en la Figura 5.21) no suman entidad suficiencia para formar un grupo con la longitud requerida o son elementos aislados y su inclusión en alguna de las cinco zonas de agrupación ocasionará un descenso del valor global de la eficiencia energética dinámica del grupo.

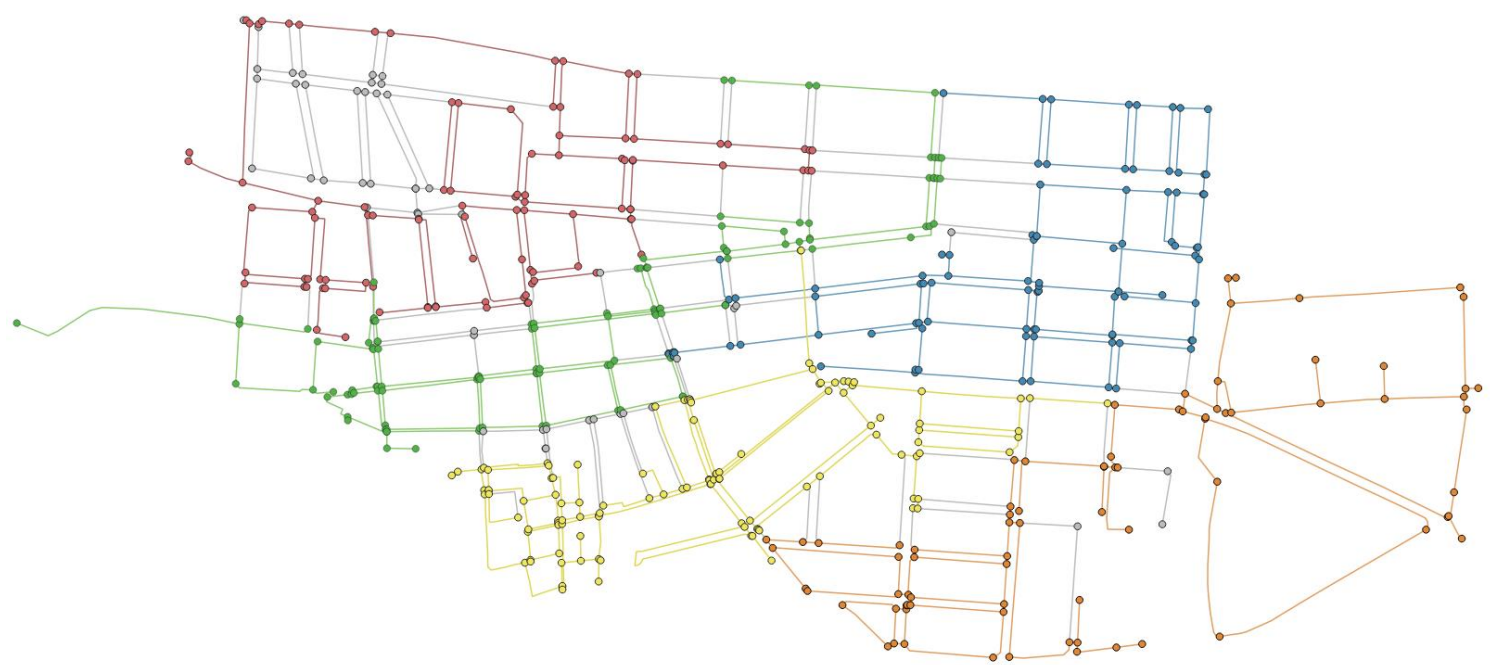

Figura 5.21 Agrupación obtenida en base a criterios energéticos 
El algoritmo de agrupación prioriza las zonas con mayor margen de mejora, estas son las que presentan menor eficiencia energética dinámica. Por ello, se puede observar (Tabla 5.30) como el orden de aparición de los diferentes grupos ha estado marcado por este criterio y hay diferencia entre cada una de las zonas. En cambio, no se observa relación entre el número de nudos o la longitud de tuberías englobas en cada uno de ellos.

Tabla 5.30 Características descriptivas de los grupos obtenidos

\begin{tabular}{|l|c|c|c|}
\hline \multicolumn{1}{|c|}{ Grupo } & $\begin{array}{c}\text { Longitud de } \\
\text { tuberías }(\mathrm{km})\end{array}$ & $\begin{array}{c}\text { Número de } \\
\text { nudos }\end{array}$ & $\begin{array}{c}\text { Eficiencia energética } \\
\text { dinámica (\%) }\end{array}$ \\
\hline 1 (naranja) & 4,8 & 79 & 37,2 \\
\hline 2 (amarillo) & 4,6 & 111 & 41,4 \\
\hline 3 (azul) & 4,6 & 94 & 43,9 \\
\hline 4 (verde) & 4,6 & 116 & 49,2 \\
\hline 5 (roja) & 4,6 & 97 & 58,8 \\
\hline
\end{tabular}

Llegados a este punto, cabe destacar que las zonas de agrupación obtenidas aún no forman los sectores definitivos, sino que son una delimitación de estos. Para ello, es necesario analizar qué tuberías se deberán dejar abiertas y cuáles se deberán cerrar, así como colocar contadores de control del agua inyectada en cada sector. Además, cabe destacar que en la red sectorizada actual cuenta con una gestión de presiones, la válvula reductora de presiones a la entrada de la Zona Baja. Por ello, a la sectorización propuesta también se complementará con una gestión de presiones. Tras ello, se propone la distribución mostrada en la Figura 5.22. Se han definido cinco sectores cuya distribución final ha venido muy marcada por la delimitación de los grupos iniciales, y se ha optado por colocar válvulas reductoras a las entradas de los sectores 2 y 4.

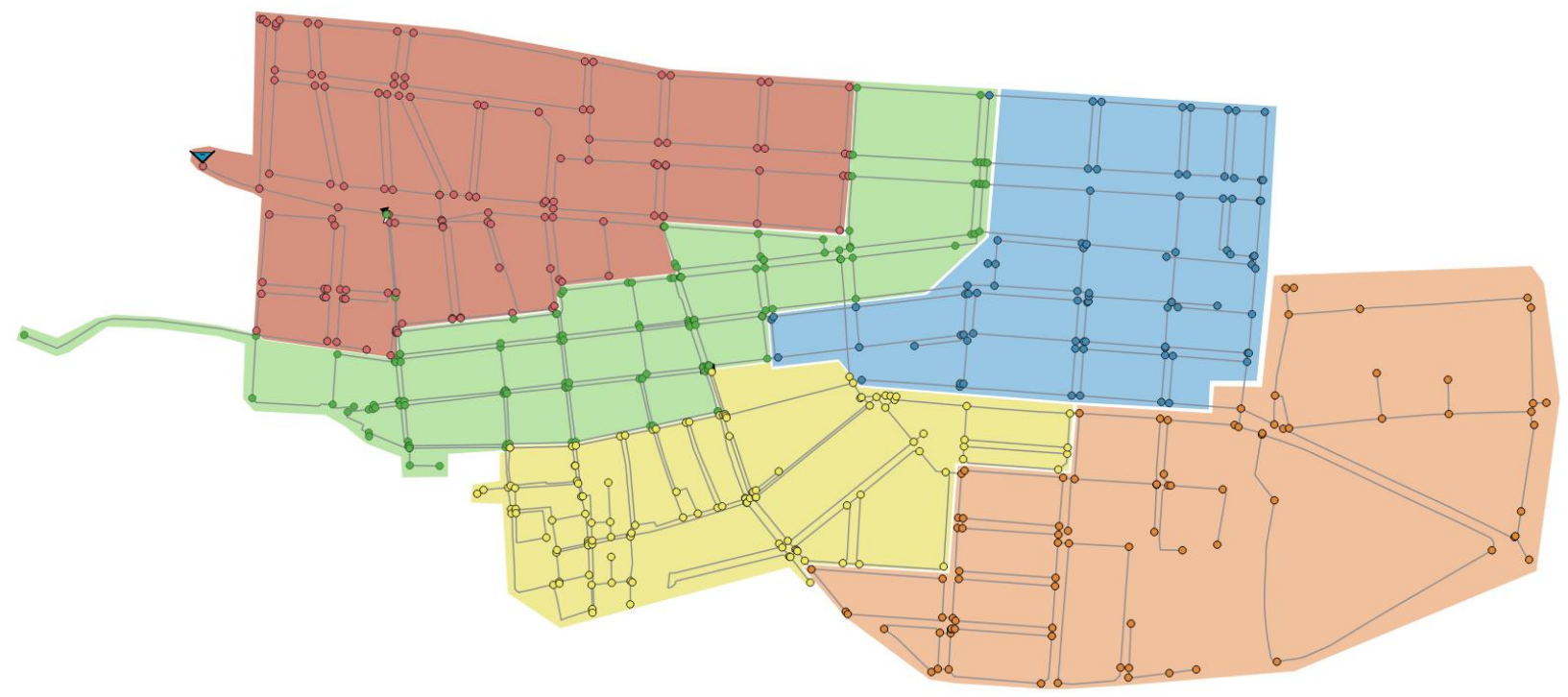

Figura 5.22 Sectorización final propuesta según criterios energéticos

Se recoge, en la Tabla 5.31, las características descriptivas principales de cada uno de los sectores propuestos siguiendo criterios energéticos. 
Tabla 5.31 Características descriptivas de los sectores según criterios energéticos

\begin{tabular}{|c|c|c|c|c|c|c|c|c|c|}
\hline \multirow{2}{*}{ Sector } & \multicolumn{2}{|c|}{ Longitud } & \multirow{2}{*}{$\begin{array}{c}\text { Diámetro } \\
\text { de } \\
\text { suministro }\end{array}$} & \multirow{2}{*}{$\begin{array}{l}\text { Gestión } \\
\text { de presión }\end{array}$} & \multicolumn{2}{|c|}{$\begin{array}{c}\text { Volumen } \\
\text { suministrado }\end{array}$} & \multicolumn{2}{|c|}{$\begin{array}{l}\text { Volumen } \\
\text { registrado }\end{array}$} & \multirow{2}{*}{$\begin{array}{c}\text { № de } \\
\text { acometidas }\end{array}$} \\
\hline & $(\mathrm{km})$ & $(\%)$ & & & $\mathrm{m}^{3} /$ día & $(\%)$ & $\mathrm{m}^{3} /$ día & $(\%)$ & \\
\hline Sector 1 & 5,6 & 18,1 & 110 & No & 133 & 16,9 & 84 & 15,5 & 178 \\
\hline Sector 2 & 5,7 & 18,4 & 150 & Sí & 124 & 15,7 & 115 & 21,3 & 398 \\
\hline Sector 3 & 5,1 & 16,4 & 160 & No & 174 & 22,0 & 104 & 19,3 & 312 \\
\hline Sector 4 & 7,1 & 23,0 & 150 & Sí & 145 & 18,4 & 114 & 21,1 & 532 \\
\hline Sector 5 & 7,4 & 24,0 & 110 & No & 213 & 27,0 & 123 & 22,9 & 372 \\
\hline
\end{tabular}

Analizando el comportamiento hidráulico de este nuevo escenario, en su estado operativo normal, se cuenta con una presión media de 37,17 mca, con valores externos de 25,87 y 49,58 mca (Figura 5.25), frente a una presión mínima de servicio de 25 mca. La velocidad promedio del agua y la pérdida de carga unitaria en las tuberías son de 0,07 m/s y 0,32 mca/ $/ \mathrm{km}$, respectivamente (Figura 5.24).

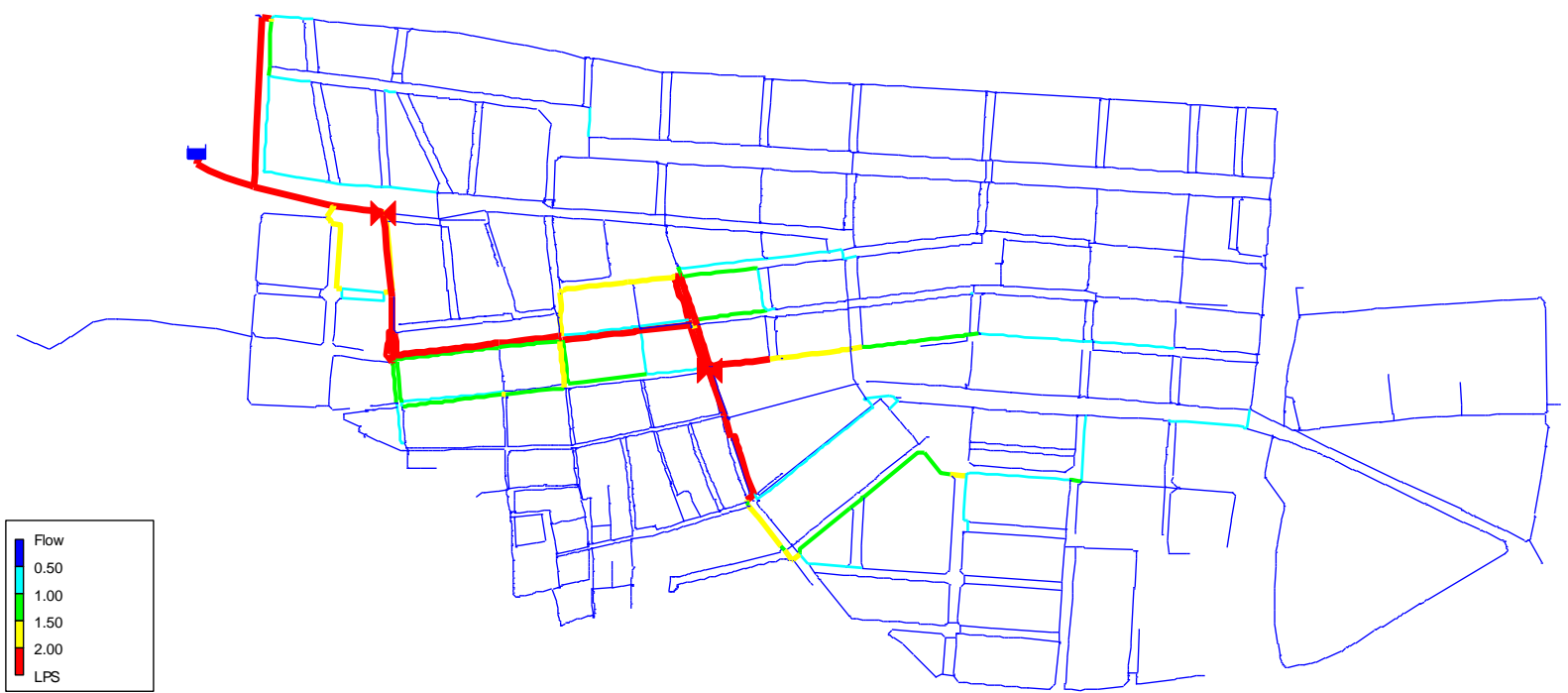

Figura 5.23 Caudales en las tuberías de distribución bajo la sectorización propuesta

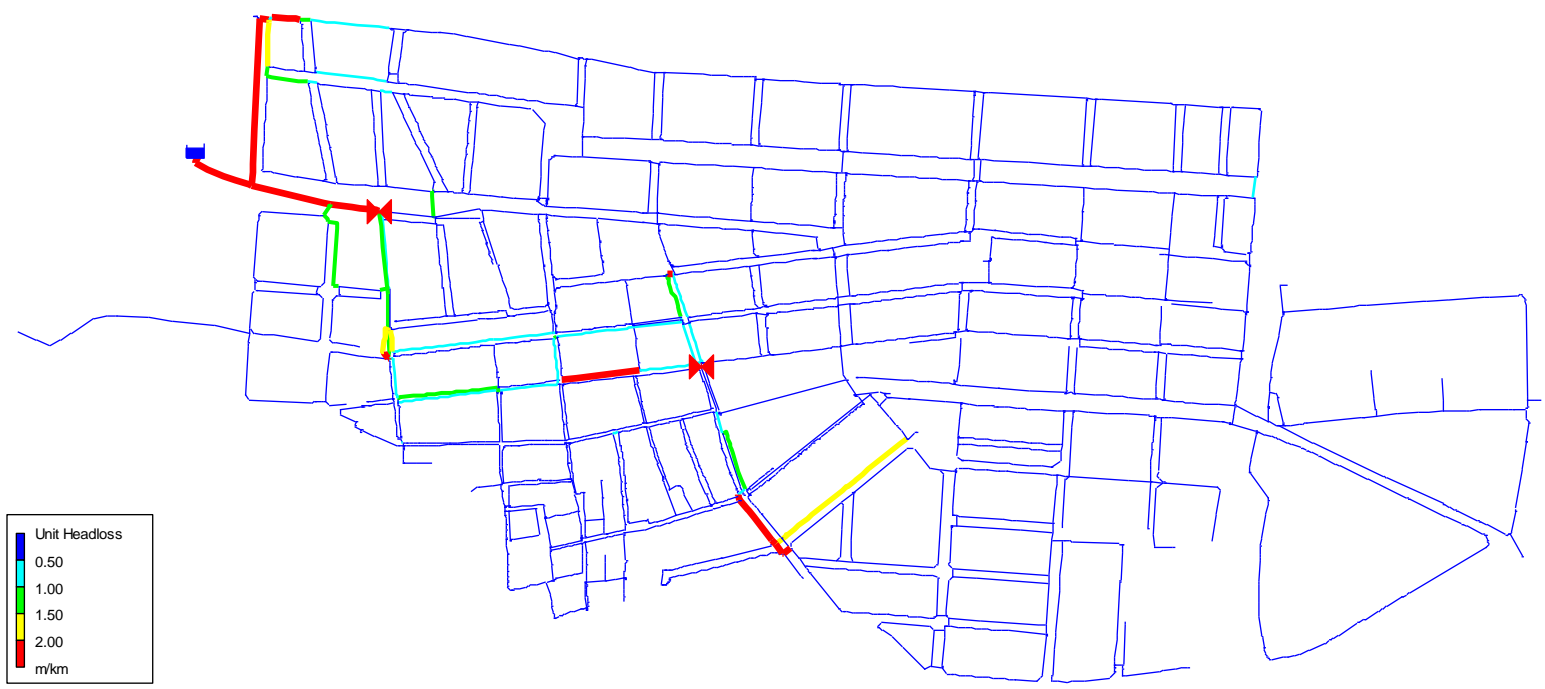

Figura 5.24 Pérdidas de carga en las tuberías de distribución bajo la sectorización propuesta 


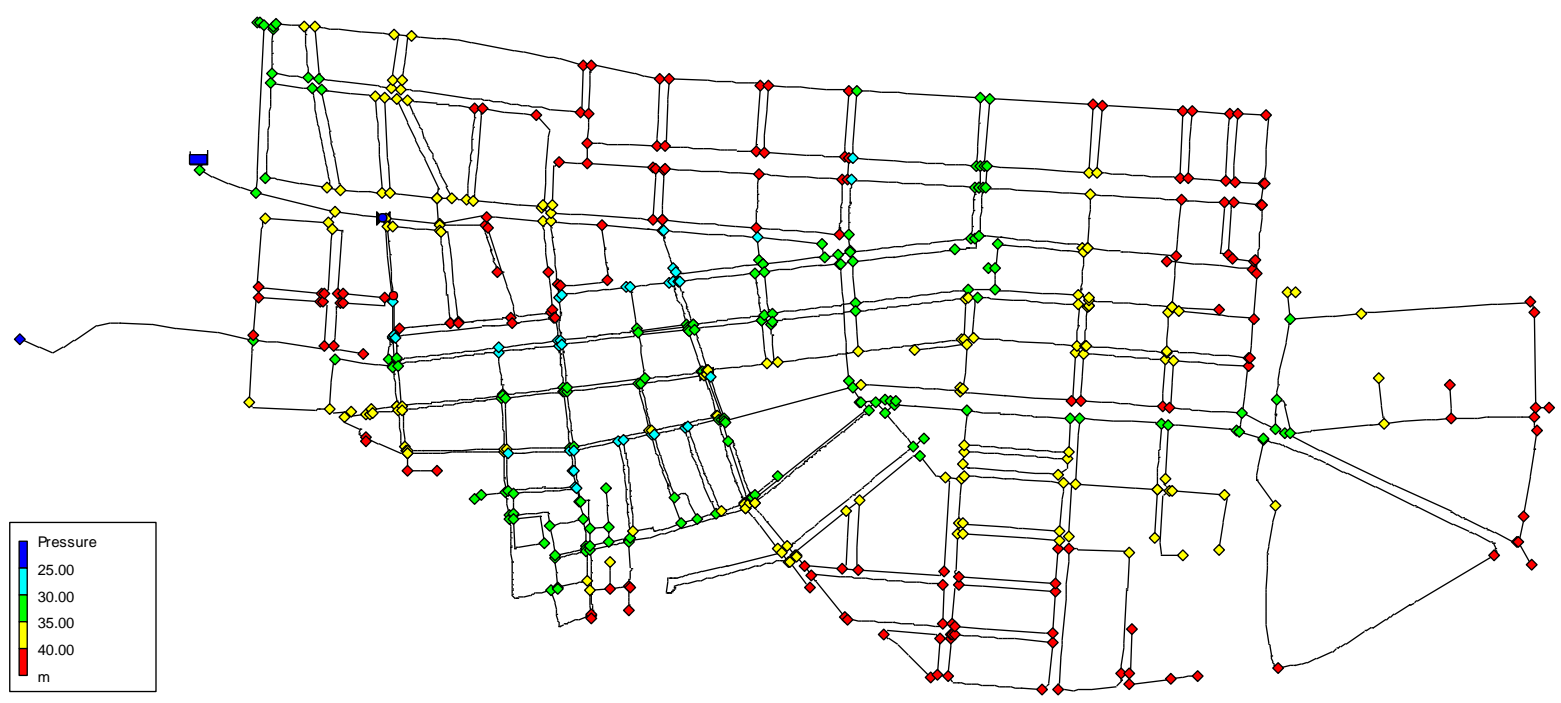

Figura 5.25 Nivel de presiones en los nudos bajo la sectorización propuesta

Entrando en mayor grado de detalle, no se desprende una gran diferencia entre sectores. Sí que se puede observar unos niveles algo inferiores en los sectores 2 y 4 , debido al empleo de la gestión de presión en la entrada de estos, pero en líneas generales los rangos de presiones son similares en los cinco sectores. En la Tabla 5.32 se recogen los principales indicadores hidráulicos de la red completa y de cada uno de los sectores.

Tabla 5.32 Balance hídrico de la red y de cada uno de los sectores según criterios energéticos

\begin{tabular}{|l|c|c|c|c|c|c|}
\hline & $\begin{array}{c}\text { Sistema } \\
\text { Completo }\end{array}$ & Sector 1 & Sector 2 & Sector 3 & Sector 4 & Sector 5 \\
\hline Caudal suministrado (m²/ía) & 786,11 & 133,04 & 123,72 & 170,70 & 145,49 & 213,16 \\
\hline Caudal fugado (m³/día) & 242,77 & 45,05 & 31,20 & 49,60 & 47,36 & 69,56 \\
\hline Presión media (mca) & 37,14 & 41,00 & 34,13 & 39,00 & 32,65 & 40,53 \\
\hline Rango presiones (mca) & $21,2-49,6$ & $32,8-48,8$ & $26,5-42,2$ & $32,3-48,6$ & $21,2-45,6$ & $29,0-49,6$ \\
\hline Caudal fugado mínimo (m³/día) & 198,13 & 35,22 & 26,63 & 39,69 & 42,15 & 54,44 \\
\hline
\end{tabular}

Desde el punto de vista energético, bajo un escenario de comportamiento normal, se entrega al sistema un total de $119,97 \mathrm{kWh} / \mathrm{d}$ (Tabla 5.33). De los cuales el $67,6 \%$ se localiza en los puntos de consumo y fugas en forma de presión, el 27,9\% se localiza en el control y regulación a través de las válvulas reductoras de presión y el 4,8\% restante se disipa durante el transporte debido a la fricción del agua con las tuberías. Todo ello, dota al sistema sectorizado según criterios energéticos de una eficiencia energética dinámica del 66\%. Entrando en detalle en cada uno de los sectores, se puede comprobar como los sectores 2 y 4 presentan unos valores de eficiencia mayores, derivado del empleo de las válvulas reductoras a las entradas de estos. Otro aspecto que destacar es la energía perdida por fricción en los sectores 2, 4 y 5. Se puede comprobar como el valor es mayor en cada uno de ellos. Esto es debido a que por el sector 2 circula el volumen suministrado al sector 1, por el sector 4 circula el de los sectores 2 y 3 y, en último lugar, por el sector 5 circula el agua suministrada a todos los anteriores. Esto hace que, en los sectores cabecera, y directamente proporcional al caudal trasegado, la pérdida de carga por fricción sea mayor.

Para terminar, es importante destacar que los valores de las eficiencias energéticas dinámicas de los cinco sectores presentan unos valores diferentes a los obtenidos en los cinco grupos 
obtenidos tras el algoritmo de agrupación ejecutado inicialmente y que sirvieron como delimitaciones iniciales de los sectores definitivos. Esto es debido, principalmente, a dos factores: el primero es el peso que aportan aquellos nudos que no se agruparon en los grupos iniciales, pero sí se han incluido en alguno de los sectores finales. El segundo, el mero hecho de la ejecución de la sectorización y la gestión de presión implantada. En los grupos inicialmente obtenidos, el punto de funcionamiento hidráulico era el de la red en condiciones originales, sin sectorizar. En los sectores definitivos, este punto de funcionamiento engloba el cierre de tuberías y la regulación de presiones. Así, los sectores 1 y 2 muestran unos valores de 62,42 y 70,84 frente a 37,2 y 41,4 de los grupos 1 y 2 . Esto es, principalmente, debido al efecto introducido por las válvulas reductoras instaladas a la entrada del sector 2 . Los grupos 3 y 4 también aumenta desde los valores de 43,9 y 49,2 hasta los 64,13 y 76,86 de los sectores 3 y 4, por la misma razón, aunque en este caso, su reducción de presión es por la válvula del sector 4 . El sector 5 aumenta ligeramente el valor frente al grupo 5, debido al efecto de la reducción global de la presión en todo el sistema y la reducción del caudal incontrolado fugado.

Tabla 5.33 Balance energético de la red con fugas tras la sectorización según criterios energéticos

\begin{tabular}{|c|c|c|c|c|c|c|c|c|}
\hline \multirow[b]{2}{*}{ RED } & \multirow[b]{2}{*}{$\begin{array}{c}\text { Demanda } \\
\left(\mathrm{m}^{3} / \mathrm{d}\right)\end{array}$} & \multicolumn{4}{|c|}{ TOTA ENERGÍA PERDIDA (kWh/d) } & \multirow{2}{*}{$\begin{array}{l}\text { Energía } \\
\text { por } \\
\text { caudal } \\
\text { unitario } \\
\left(\mathrm{kWh} / \mathrm{m}^{3}\right)\end{array}$} & \multirow{2}{*}{$\begin{array}{l}\text { Mín. } \\
\text { energía en } \\
\text { grifo de } \\
\text { consumidor } \\
\text { (kWh/d) }\end{array}$} & \multirow{2}{*}{$\begin{array}{c}\text { Ef. } \\
\text { energética } \\
(\%)\end{array}$} \\
\hline & & Fricción & Control & $\begin{array}{c}\text { Grifo } \\
\text { consumidor }\end{array}$ & Total & & & \\
\hline $\begin{array}{l}\text { Sectorización } \\
\text { energética }\end{array}$ & 786,24 & 5,76 & 33,12 & 81,15 & 119,97 & 0,10 & 53,56 & 66,00 \\
\hline Sector 1 & 133,04 & 0,01 & - & 14,52 & 14,53 & 0,11 & 9,06 & 62,42 \\
\hline Sector 2 & 123,91 & 0,34 & 5,04 & 11,92 & 12,25 & 0,10 & 8,44 & 70,84 \\
\hline Sector 3 & 169,80 & 0,01 & - & 18,04 & 18,05 & 0,11 & 11,57 & 64,13 \\
\hline Sector 4 & 149,09 & 0,95 & 28,08 & 13,21 & 14,16 & 0,14 & 10,16 & 76,86 \\
\hline Sector 5 & 209,57 & 4,45 & - & 23,34 & 27,80 & 0,11 & 14,28 & 61,16 \\
\hline
\end{tabular}

Para concluir con este apartado, se compararán los resultados obtenidos con la sectorización según conceptos energéticos con los de la sectorización actual del caso de estudio (apartado 5.3.2.1). El primer aspecto por considerar es el nivel de presiones en la red. Por normal general, al sectorizar una red se está introduciendo pérdidas de carga que conllevan reducciones en el nivel medio de presiones, pero se puede comprobar como esta reducción depende de la disposición de dicha sectorización. En el caso de la sectorización energética, el nivel medio de presiones ha descendido en un 16,63\%, pasando de 44,55 mca hasta los 37,14 mca. Este efecto se agudiza en los valores máximos, descendiendo de los 75,12 mca hasta los 49,6 mca. Cabe recordar que, en ambos escenarios, además de contar con la red sectorizada también se cuenta con una gestión de la presión. Este efecto de la reducción de la presión ocasiona una reducción directa del volumen fugado, con una reducción de este del $10,04 \%$. Desde el punto de vista topológico, se pasa de una sectorización con 2 sectores, con unos tamaños del $60,7 \%$ y $39,3 \%$ de longitud de red y un promedio de 900 acometidas por sector, a 5 sectores con una longitud y número de acometidas promedio de 20,4\% y 358 , respectivamente. Desde el punto de vista energético, y nivel global, la sectorización según criterios energéticos requiere un 3,45\% menos de energía y presenta una eficiencia energética dinámica un $24,04 \%$ mayor que en el escenario inicial. Desglosando estos números, se puede observar como la sectorización según criterios energéticos requiere más energía en la fricción 
y el control y regulación del sistema, debido al mayor número de válvulas cerradas y válvulas de regulación de la presión, disponiendo menor energía en el punto de consumo frente al escenario actual.

A nivel sectorial, no tiene mayor sentido realizar comparaciones entre los sectores obtenidos entre ambos escenarios, al tener diferentes topologías y puntos de funcionamiento. Pero sí se puede realizar una comparativa entre los parámetros hidráulicos de todos los nudos bajo diferentes escenarios. Así, en la Tabla 5.34 se recogen los valores de presiones y volúmenes de los nudos de los cinco sectores de la sectorización según criterios energéticos, pero con los valores de estos para el escenario inicial. Comparando esta tabla con la Tabla 5.32 se puede observar como de manera global, todos los grupos de nudos de los primeros cuatro sectores tiene un comportamiento similar: mayores presiones medias, que implica un mayor volumen fugado y por lo tanto suministrado y unos rangos de presiones más amplios y con mayores valores mínimos y máximos. Respecto al sector 5 , se puede observar cómo es mínima la diferencia entre sectores debido a su disposición en el sistema.

Tabla 5.34 Balance hídrico de cada uno de los sectores del $2^{\circ}$ escenario según el funcionamiento inicial

\begin{tabular}{|l|c|c|c|c|c|}
\hline & Sector 1 & Sector 2 & Sector 3 & Sector 4 & Sector 5 \\
\hline Caudal suministrado (m³/día) & 139,01 & 124,91 & 181,26 & 154,34 & 213,44 \\
\hline Caudal fugado (m/día) & 51,02 & 32,40 & 60,16 & 56,52 & 69,85 \\
\hline Presión media (mca) & 50,08 & 36,75 & 57,36 & 42,73 & 41,26 \\
\hline Rango presiones (mca) & $35,64-75,12$ & $28,91-56,43$ & $50,62-67$ & $21,16-55,98$ & $32,3-49,51$ \\
\hline Caudal fugado mínimo (m²/ía) & 35,22 & 26,63 & 39,69 & 42,15 & 54,44 \\
\hline
\end{tabular}

Siguiendo esta línea, también se puede realizar una comparativa similar entre el comportamiento de los nudos de la sectorización según criterios energéticos y los mismos antes de realizar cualquier sectorización (Tabla 5.35). En este caso, se observa el mismo comportamiento de los primeros cuatro sectores, pero con unas diferencias aún mayores. En el caso del sector 5 , se sigue observando un comportamiento similar entre escenarios.

Tabla 5.35 Balance hídrico de cada uno de los sectores del 2ํe escenario según el funcionamiento sin sectorizar

\begin{tabular}{|l|c|c|c|c|c|}
\hline & Sector 1 & Sector 2 & Sector 3 & Sector 4 & Sector 5 \\
\hline Caudal suministrado (m³/día) & 145,68 & 133,77 & 181,18 & 157,35 & 213,34 \\
\hline Caudal fugado (m/día) & 57,69 & 41,26 & 60,08 & 59,21 & 69,75 \\
\hline Presión media (mca) & 67,10 & 59,76 & 57,21 & 50,41 & 41,15 \\
\hline Rango presiones (mca) & $58,94-74,97$ & $52,05-70,81$ & $50,47-66,85$ & $39,15-61,53$ & $32,2-49,41$ \\
\hline Caudal fugado mínimo (m³/día) & 35,22 & 26,63 & 39,69 & 42,15 & 54,44 \\
\hline
\end{tabular}

\subsubsection{Sensorización para la localización de fugas}

A continuación, se aplicará sobre la red de estudio el uso de sensórica para la localización de posibles consumos incontrolados, ya sean fugados o consumos no autorizados. Esta técnica, aunque puede ser complementaria a la sectorización, se ha presentado a lo largo de este estudio como una metodología alternativa para la localización de las fugas. Por ello, se aplicará sobre la red original sin estar sectorizada ni contando con una gestión de presiones. 
Para aplicar dicha metodología es necesario conocer qué caudal se está escapando del sistema sin ser controlado, que en este caso se fija en $30 \mathrm{l} / \mathrm{min}$, y qué red de sensores se tienen instalados, así como sus lecturas registradas. Con el objetivo de analizar el alcance de esta metodología de búsqueda para este caso de estudio, se analizarán diferentes distribuciones de sensores. Así, se ha elegido un primer escenario con 20 sensores de presión distribuidos de manera uniforme (configuración 1) y un segundo con 40 sensores de presión y 5 caudalímetros, ubicados igualmente que el escenario anterior (configuración 2).

Una vez conocidos los datos de partida, y como primer paso, se realizará un análisis de sensibilidad para este escenario. Como resultado se obtendrá la variación media sufrida en la altura piezométrica de los nudos y los caudales circulantes por las tuberías. En el caso de los nudos, se desprende que todos presentan una sensibilidad ante el cambio muy similar. Ante una fuga de $30 \mathrm{l} / \mathrm{min}$, se produce una disminución media en la presión de todos los nudos con un valor medio de 0,24 mca con una desviación de 0,02 mca (Figura 5.26). Estos valores son significativos de una infraestructura mallada y sobredimensionada, en línea al análisis hidráulico realizado en el apartado 5.3.2.1. Aun así, se pueden observar varios nudos cuyo comportamiento se aleja del valor medio. Es el caso de los nudos que se encuentran junto al depósito de cabecera, y por ello su valor de presión no se ve alterado, o el J18, cuya disposición (largo ramal a la izquierda del sector Zona Alta, Figura 5.17) hace que la distribución típica de todas las variaciones sean algo mayor.

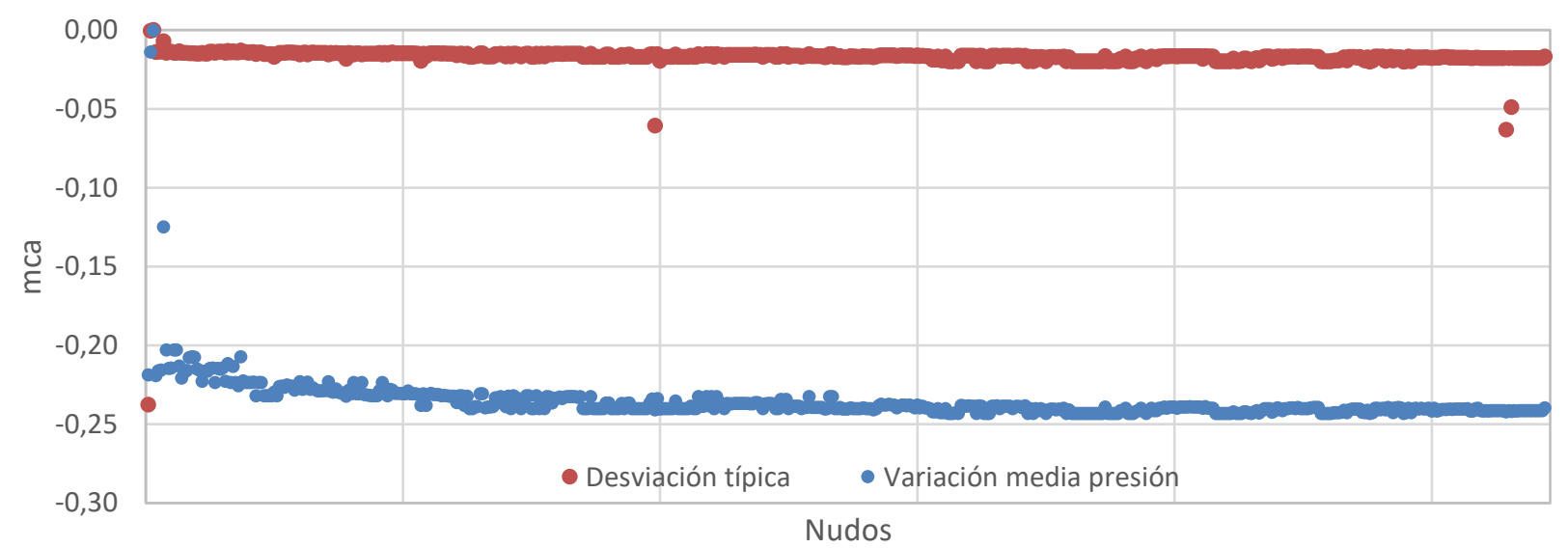

Figura 5.26 Variaciones medias de presión (mca) y sus desviaciones en los nudos ante una fuga de 30 $1 / \min$

Para terminar de profundizar sobre el análisis de la sensibilidad de los nudos, véase cómo varía la presión en el nudo J18 (eje de ordenadas en la Figura 5.27) al ir variando la ubicación de la fuga de $30 \mathrm{l} / \mathrm{min}$ a cada uno de los nudos de la red (eje de abscisas en la Figura 5.27). Este nudo se alimenta a través de una tubería de $32 \mathrm{~mm}$ de diámetro y una longitud de 266 metros. Esto hace que, en el caso de producirse la fuga en dicho nudo, la presión del nudo baje hasta los 33,4 mca y que, a su vez, este valor se vea afectado cuando la localización de la fuga se produzca en nudos cercanos. En contra, si la localización se produce en nudos más alejados, la presión del nudo J18 se ve mínimamente afectada. 


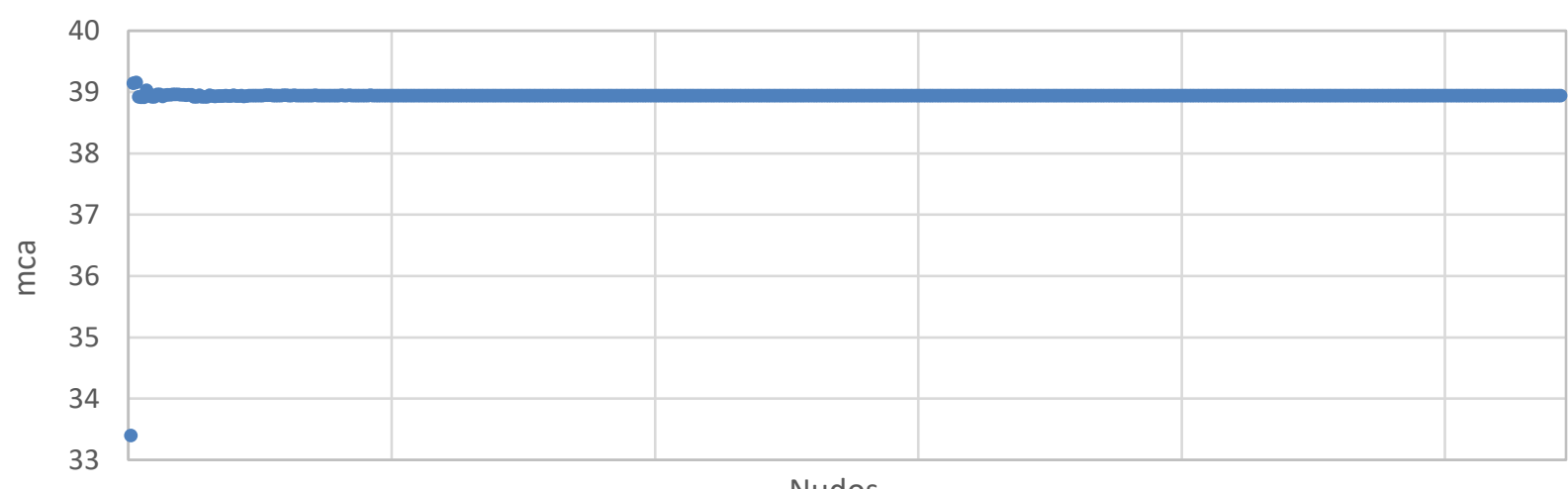

Nudos

Figura 5.27 Evolución de la presión en el nudo J18 ante una fuga de $30 \mathrm{l} / \mathrm{min}$ en cada uno de los nudos de la red

Nótese que en la Figura 5.27 se recogen todos los valores de la presión del nudo J18 para todas las ubicaciones posibles de un consumo adicional de $30 \mathrm{l} / \mathrm{min}$, y para ello es necesario realizar $n$ resoluciones de los $n$ escenarios diferentes. La metodología recogida en el capítulo 4, obtiene de manera directa la variación media de la presión para el consumo adicional dado. Evidentemente, para ello es necesario realizar una serie de suposiciones, pero la diferencia entre hacerlo de manera directa u obtener la presión media a través de las $n$ simulaciones es del orden de 0,01 mca, en el nudo J18, y de 0,002 mca como valor medio de todos los nudos.

Para los caudales circulantes, sí que se obtienen mayores discrepancias entre todas ellas (Figura 5.28), estando relacionada la variación sufrida con el nivel de caudal inicial, el cual está fuertemente relacionado con el diámetro de cada una de las tuberías. Siendo la variación del caudal medio en las tuberías es de 1,15 l/min con una desviación de 2,00, con valores que se mueven entre los 0,03 y $29,54 \mathrm{l} / \mathrm{min}$.

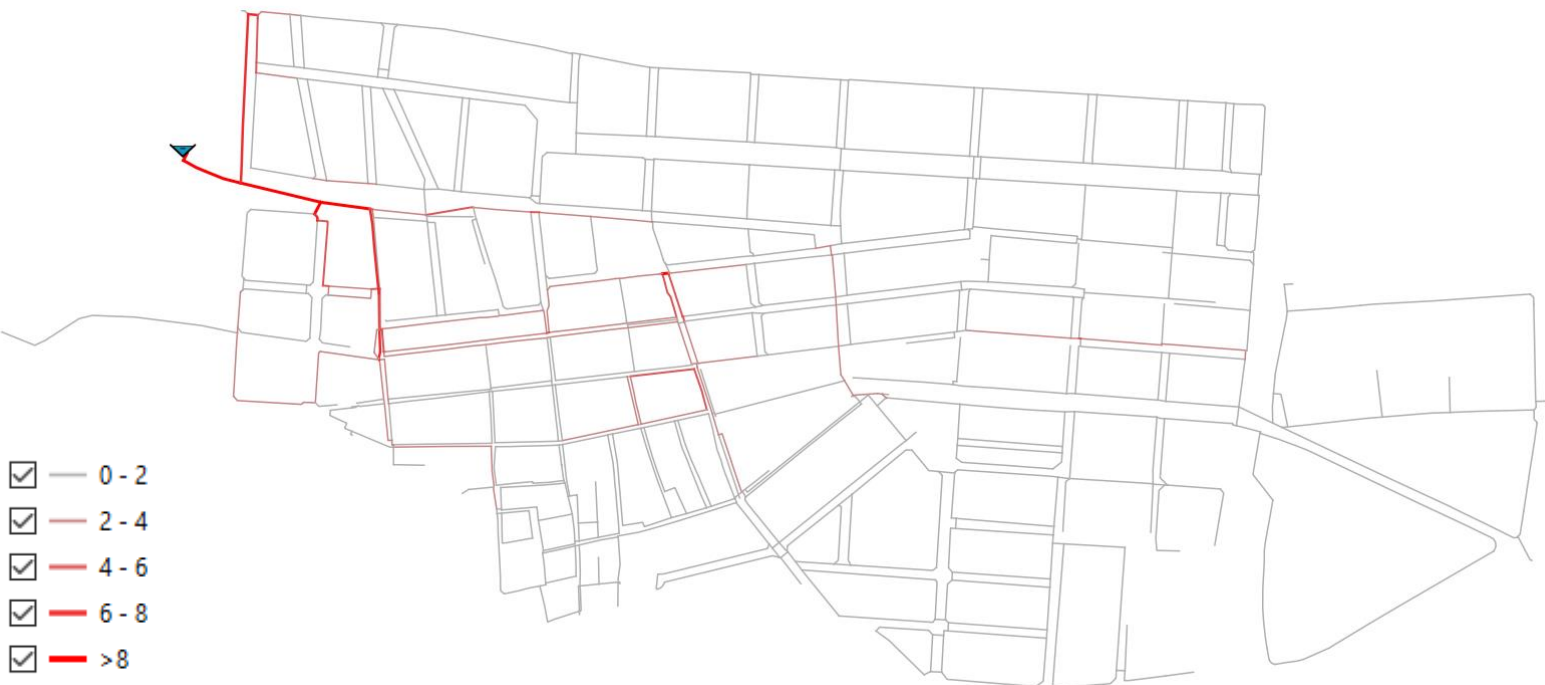

Figura 5.28 Variaciones de caudal (I/min), en valores absolutos, en las líneas ante una fuga de $30 \mathrm{l} / \mathrm{min}$

Una vez realizado el análisis de sensibilidad, y obtenido las variaciones medias de presión y caudal en los nudos y tuberías, el siguiente paso es obtener las lecturas de los sensores para poder realizar la localización de la fuga.

Para este caso, se contará inicialmente con una distribución de 20 sensores de presión ubicados de manera homogénea por la red (Figura 5.29). Se ha elegido una distribución 
homogénea de los mismos en base a los resultados del análisis de sensibilidad, donde se observaba un comportamiento similar de todos ellos.

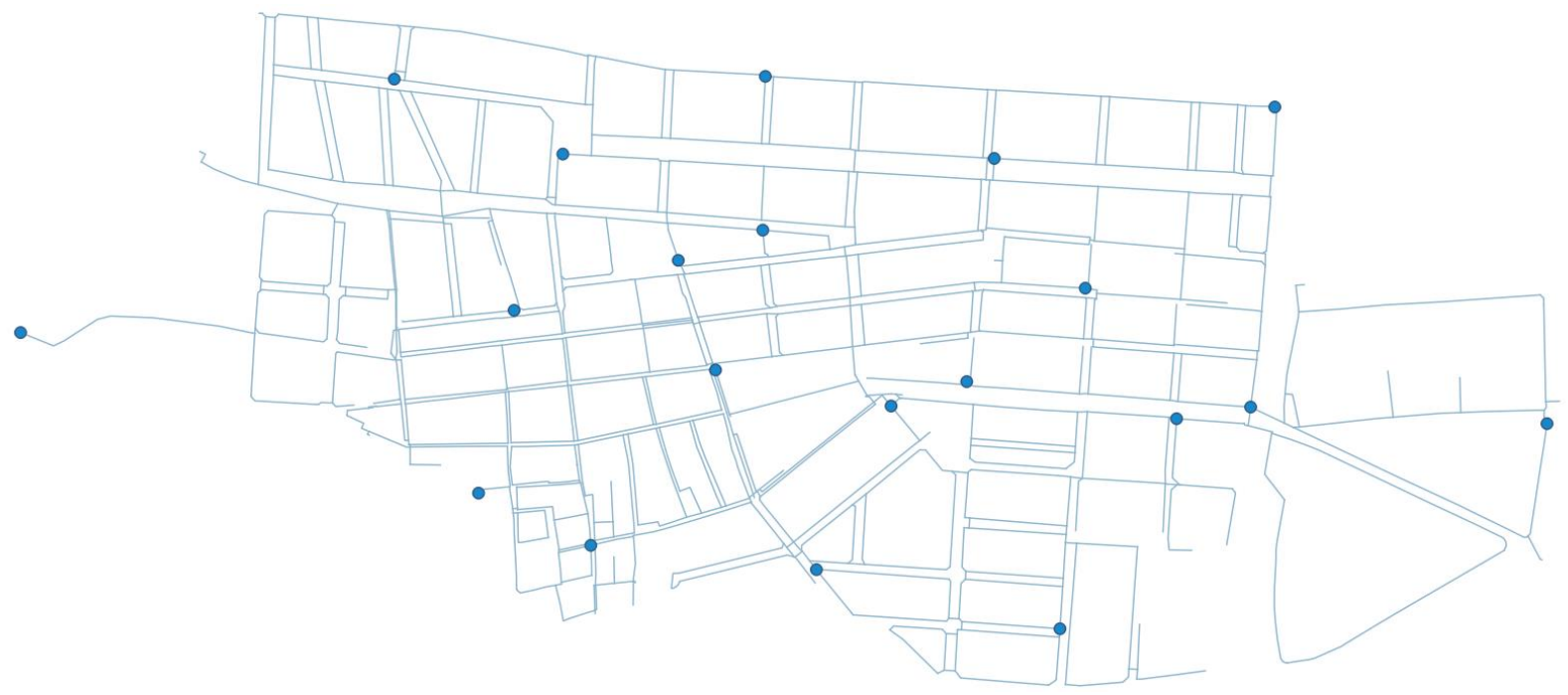

Figura 5.29 Distribución homogénea de los 20 sensores de presión

Los datos de presión recogidos por estos 20 sensores son datos reales, y entrarán en el modelo de cálculo como valores conocidos. Y el resto de los valores de presiones, que también deben que establecerse como valores conocidos, se estimarán como el valor del escenario inicial más la variación de presión media sufrida en cada nudo para el incremento de consumo fijado.

Con todo ello, se simula una fuga de $30 \mathrm{l} / \mathrm{min}$ en el nudo $\mathrm{J} 67$ (redondeado en verde en la Figura 5.30). Con los datos obtenidos por los sensores, se simula el modelo desarrollado en el capítulo 4, obteniendo como resultado el caudal fugado en cada nudo. En un caso ideal, donde se contará con un medidor de presión en cada punto de consumo, se obtendría un consumo extra tan solo en el nudo J67. Pero conforme se ve reducido el número de sensores, las hipótesis de cálculo van tomando relevancia y, con ello, la zona de localización aumenta. Actualmente tan solo se cuenta con 20 sensores de presión repartidos por la red, lo cual supone una cobertura del 3,7\% del total de nudos. Con esta disposición, en la Figura 5.30 se muestra en cada nudo el caudal de fuga que desprende el modelo, despreciando aquellos cuyo valor asignado es cercano a cero. 


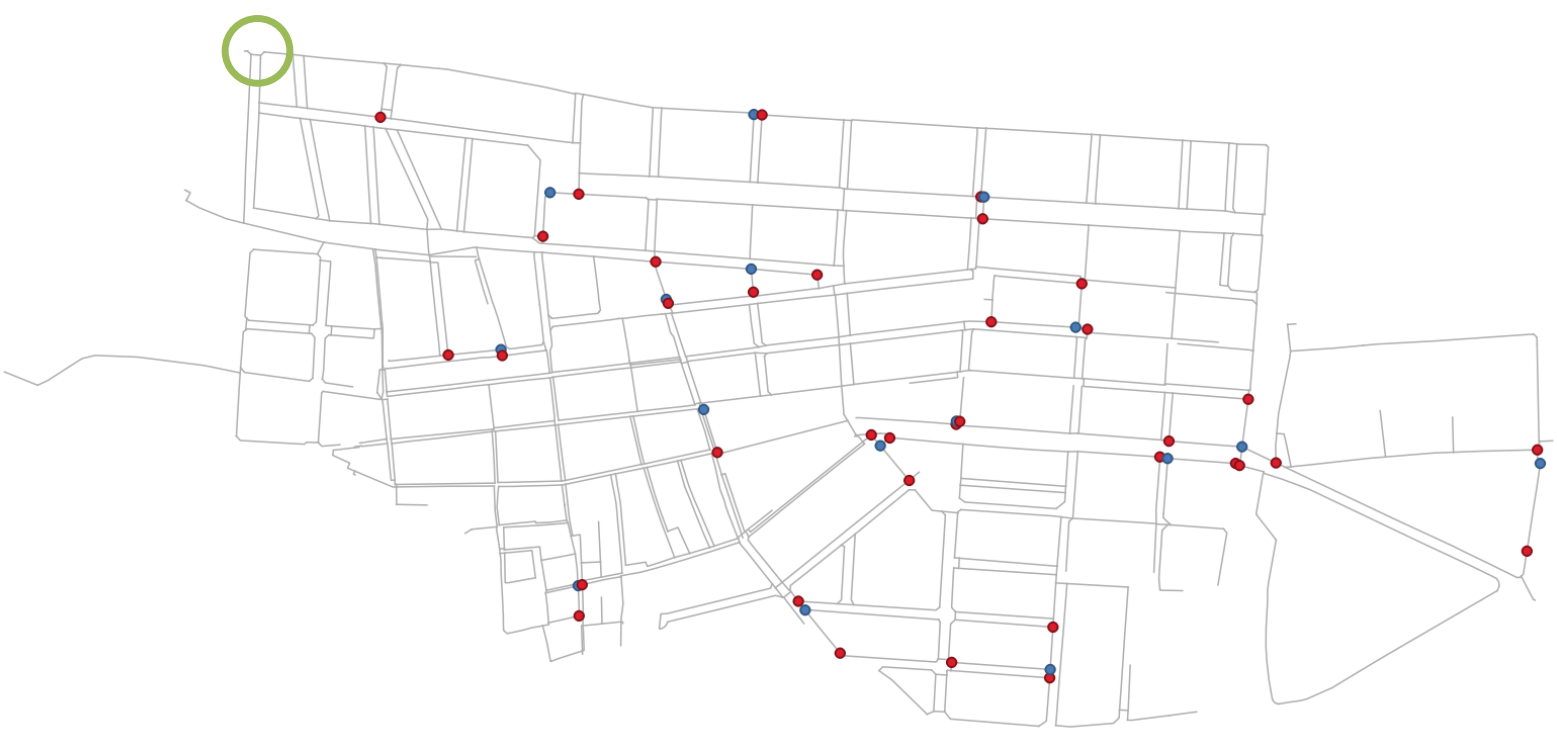

Figura 5.30 Ubicación real de la fuga (verde) y resultado del modelo de localización (azul y rojo)

A primera vista se podría considerar que la localización no ha sido la esperada, pero si analizamos con detalle los resultados obtenidos se puede comprobar que el modelo ha asignado a ciertos nudos (marcados en azul en la Figura 5.30) un caudal fugado negativo, es decir, en estos nudos se está produciendo una entrada de agua. Si analizamos alguno de ellos, por ejemplo, el J710 (Figura 5.31), se puede comprobar que el caudal de fuga que entra al sistema por el nudo J710 sale por los tres nudos colindantes. Esto es debido a que la presión del nudo J710 introducida en el modelo es la real, al contar con un sensor de presión. Y la única manera de ajustar este valor con el valor de las presiones estimadas en sus nudos colindantes es asignando caudales consumidos negativos. Sumando los cuatro valores se puede comprobar como el balance da un caudal fugado para cada nudo muy cercano a cero, y por lo tanto se pueden excluir del resultado.

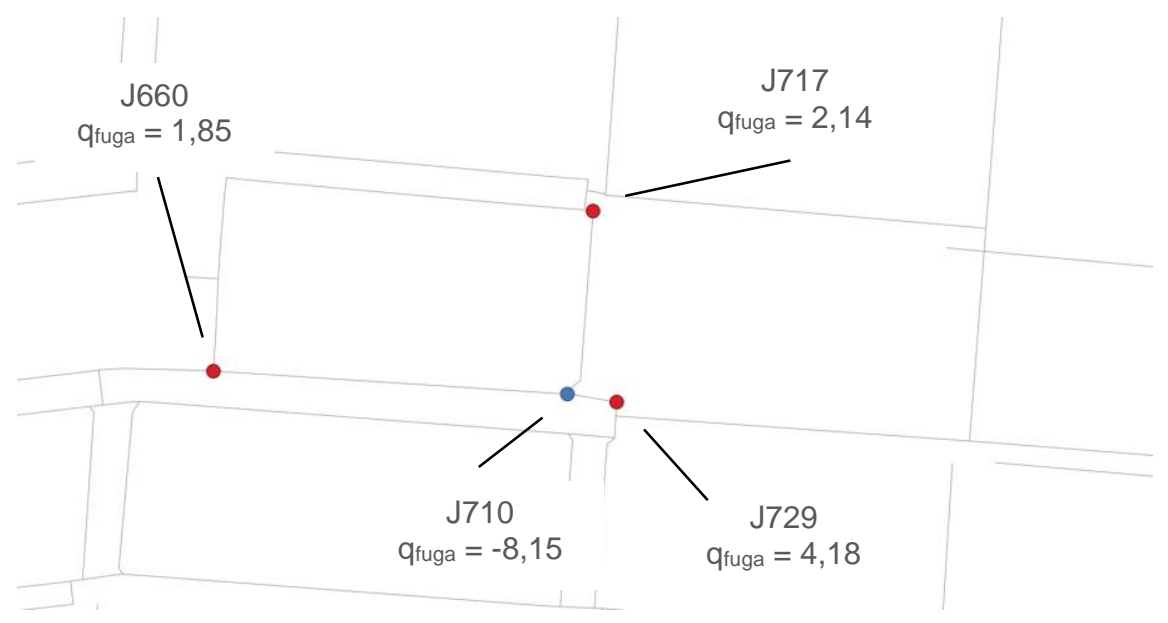

Figura 5.31 Resultado del modelo sobre el nudo J710

Ahora bien, aunque desde el punto de vista teórico sea admisible un caudal de fuga entrando al sistema, prácticamente no puede darse. Por ello, si solo destacamos aquellos nudos equipados con sensor, el resultado de la localización queda más claro. En la Figura 5.32 se puede apreciar cómo, de todos los sensores de presión, el más cercano al nudo J67 marca un consumo de fuga. Cuantitativamente, el modelo asigna un caudal fugado en dicho sensor de $101 \mathrm{l} / \mathrm{min}$. Este valor está alejado de los $30 \mathrm{l} / \mathrm{min}$ de la fuga simulada, y la discrepancia 
vendrá marcada por la distancia entre la ubicación exacta y el sensor en cuestión. Aunque es importante destacar la utilizada de la localización de la zona donde se está produciendo la fuga, más que el valor en sí obtenido por el modelo.

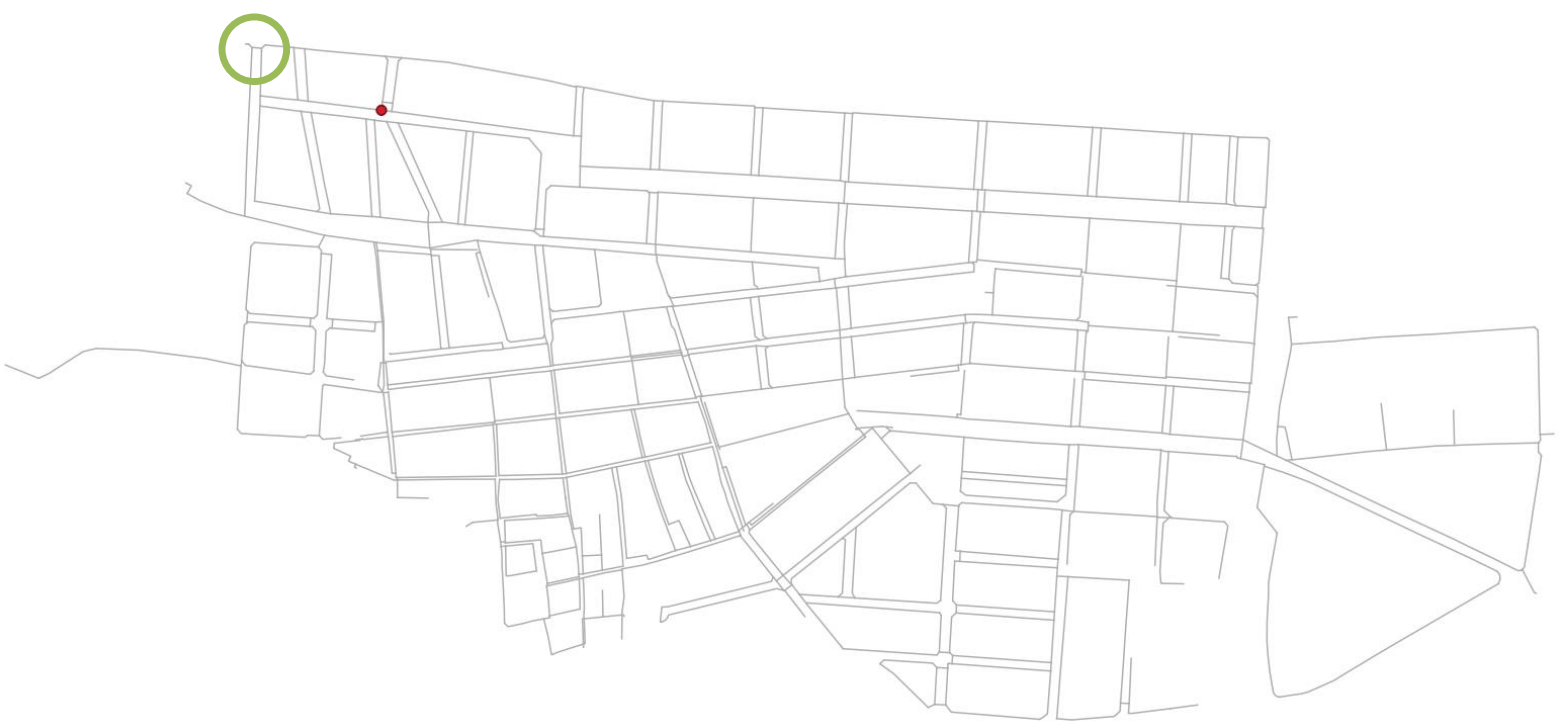

Figura 5.32 Ubicación real de la fuga (verde), nudo J67, y sensores con caudal fugado asignado (rojo)

Bajo el mismo escenario, el grado de acierto en la localización dependerá de la ubicación real de la fuga. Se muestran, como ejemplo, qué pasaría si la fuga se localizara en el nudo J254 (Figura 5.33) y en el nudo J465 (Figura 5.34). Esto demuestra que la facilidad en la localización depende en gran medida, no solo del número de sensores, sino de la ubicación de estos y la topología de la red.

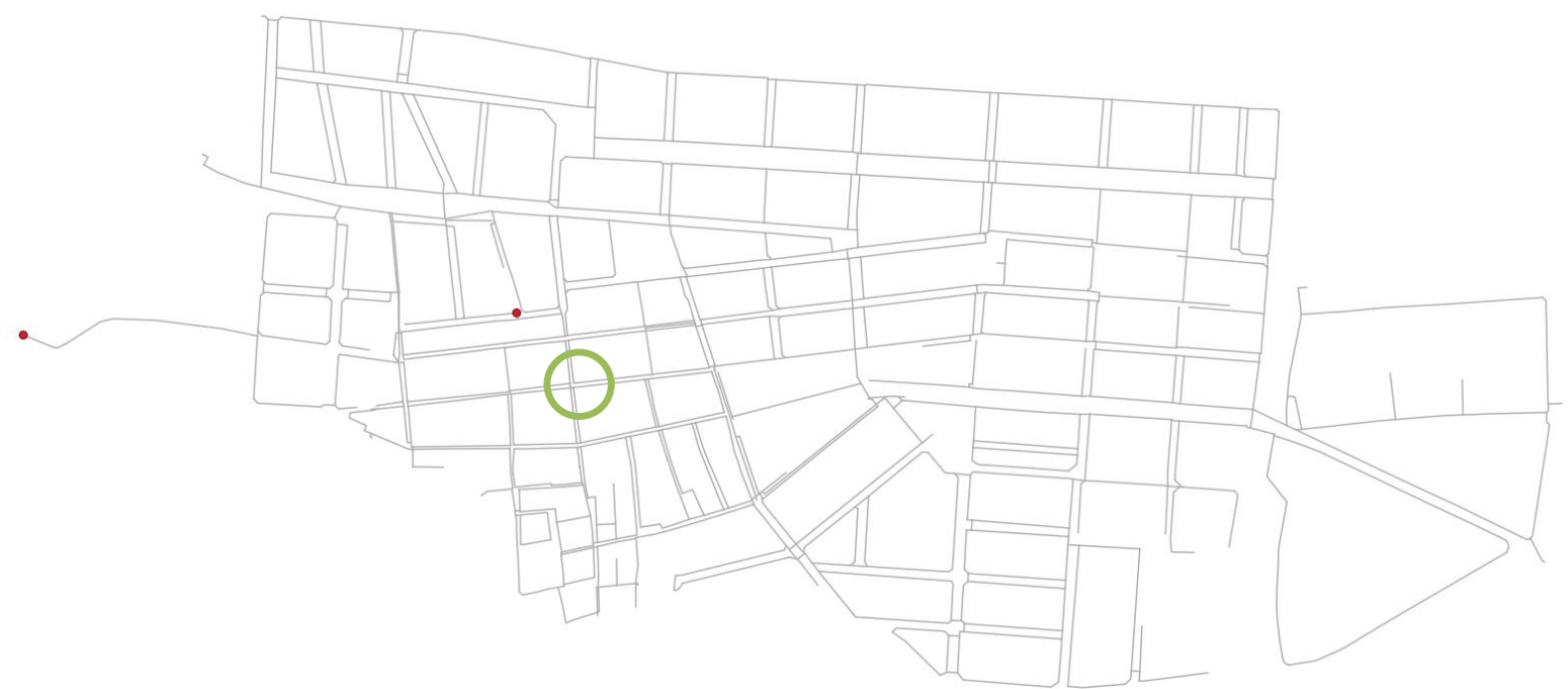

Figura 5.33 Ubicación real de la fuga (verde), nudo J254, y sensores con caudal fugado asignado (rojo) 


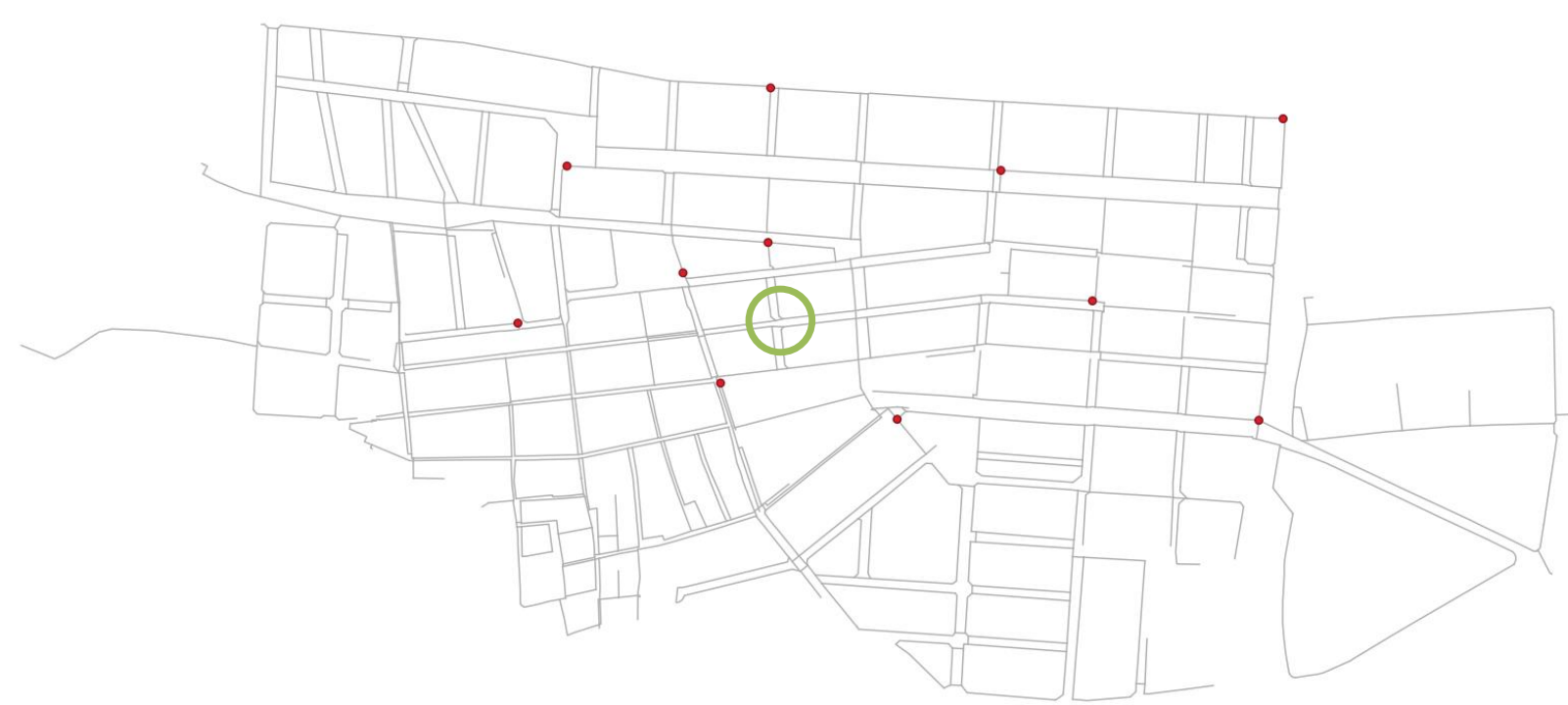

Figura 5.34 Ubicación real de la fuga (verde), nudo J465, y sensores con caudal fugado asignado (rojo)

A diferencia del caso 1, en este caso se observa con mayor facilidad el resultado que desprende la metodología planteada. Se puede observar cómo, para cada planteamiento, la zona detectada cambia según el entorno. Así, para la configuración 1 propuesta, se obtiene una longitud de búsqueda promedio de 8,38 kilómetros, reduciéndose hasta los 5,85 kilómetros para la configuración 2 (Tabla 5.36).

Tabla 5.36 Tabla resumen para la configuración de sensores probadas en el estudio de caso 2

\begin{tabular}{|c|c|c|}
\hline & Config. 1 & Config. 2 \\
\hline № se sensores de presión & 20 & 40 \\
\hline № de caudalímetros & 0 & 5 \\
\hline Número escenarios & 546 & 546 \\
\hline $\begin{array}{c}\text { Ubicación de la fuga en el } \\
\text { sensor cercano }\end{array}$ & 114 & 109 \\
\hline Detección del área & 541 & 541 \\
\hline No detección & 5 & 5 \\
\hline $\begin{array}{c}\text { Longitud de búsqueda } \\
\text { promedio (km) }\end{array}$ & 8,38 & 5,85 \\
\hline
\end{tabular}

\subsubsection{Efectividad y eficiencia en la búsqueda de fugas no declaradas}

Para este segundo caso, se plantea una planificación de las intervenciones de tal forma que se igualen los costes de pérdidas de agua con los de correlación de la red (Hunaidi, 2005). Según esta política, la frecuencia de intervención y el tiempo de correlación de la red inicial quedan recogidos en la tabla Tabla 5.37.

Tabla 5.37 Frecuencia de intervención y tiempo de correlación para la red inicial

\begin{tabular}{|c|c|c|c|}
\hline Escenario & $\begin{array}{c}\text { Frecuencia de } \\
\text { intervención (días) }\end{array}$ & $\begin{array}{c}\text { Tiempo de correlación } \\
\text { (jornadas) }\end{array}$ & $\begin{array}{c}\text { Velocidad de inspección } \\
\text { (m/h) }\end{array}$ \\
\hline Red inicial & 159,2 & 12,83 & 300 \\
\hline
\end{tabular}

Esta política de intervención supondrá un coste anual por las campañas de intervención y el agua fugada por un total de $22.089 €$ €/año (Hunaidi, 2005). 
En el caso de la sectorización según criterios energéticos, y siguiendo la misma política de intervención, el coste anual será de $20.950 €$ €año, según la frecuencia de intervención y el tiempo de correlación recogidas en la Tabla 5.38.

Tabla 5.38 Frecuencia de intervención y tiempo de correlación para la sectorización según criterios energéticos

\begin{tabular}{|c|c|c|c|}
\hline Escenario & $\begin{array}{c}\text { Frecuencia de } \\
\text { intervención (días) }\end{array}$ & $\begin{array}{c}\text { Tiempo de correlación } \\
\text { (jornadas) }\end{array}$ & $\begin{array}{c}\text { Velocidad de inspección } \\
\mathbf{( m / h )}\end{array}$ \\
\hline Red inicial & 167,85 & 12,83 & 300 \\
\hline
\end{tabular}

En el caso de usar la sensórica para la localización y ubicación de las fugas, y debido a que la política de intervención seguida es independiente de la organización de la red, se tendrán los mismos valores que la situación inicial para los trabajos de inspección de la red, pero mayores debido al agua perdida, al ser mayores los niveles fugados por una mayor presión media.

\subsubsection{Capacidad hidráulica de la red resultante}

Para analizar la capacidad hidráulica de la red, se seguirá la misma línea que la seguida en el caso de estudio 1. Se empezará analizando los datos de presiones obtenido en los tres escenarios analizados, y cuyos valores se recogen en la Tabla 5.39.

Tabla 5.39 Niveles de presiones

\begin{tabular}{|c|c|c|c|}
\hline Indicador & $\begin{array}{c}\text { Sectorización } \\
\text { inicial }\end{array}$ & $\begin{array}{c}\text { Sectorización } \\
\text { energética }\end{array}$ & $\begin{array}{c}\text { Red } \\
\text { Sensorizada }\end{array}$ \\
\hline Presión media (mca) & 44,50 & 37,11 & 53,93 \\
\hline Presión máxima (mca) & 75,10 & 49,58 & 74,97 \\
\hline$P_{\text {max }} / P_{\text {media }}(-)$ & 1,69 & 1,34 & 1,39 \\
\hline Presión mínima (mca) & 21,16 & 21,20 & 32,20 \\
\hline$P_{\text {min }} / P_{\text {media }}(-)$ & 0,48 & 0,57 & 0,60 \\
\hline$P_{\text {media }} / P_{\text {min admisible }}(-)$ & 2,23 & 1,86 & 2,69 \\
\hline
\end{tabular}

Siguiendo con el nivel de presiones, se van a añadir los indicadores exceso de presión media, déficit de presión y desviación de la presión respecto a la presión de diseño. De esta menara, se obtienen los valores mostrados en la Tabla 5.40 para los diferentes escenarios.

Tabla 5.40 Exceso de presión media, déficit de presión y desviación de la presión

\begin{tabular}{|c|c|c|c|}
\hline Indicador & $\begin{array}{c}\text { Sectorización } \\
\text { inicial }\end{array}$ & $\begin{array}{c}\text { Sectorización } \\
\text { energética }\end{array}$ & $\begin{array}{c}\text { Red } \\
\text { Sensorizada }\end{array}$ \\
\hline Exceso de presión media (mca) & 27,10 & 17,93 & 33,93 \\
\hline Déficit de presión (mca) & 0 & 0 & 0 \\
\hline $\begin{array}{c}\text { Desviación de la presión respecto } \\
\text { a la presión de diseño (mca) }\end{array}$ & 26,50 & 18,04 & 35,31 \\
\hline
\end{tabular}

Para terminar con el nivel de presiones, se calculará el índice HP, con las mismas consideraciones que en el caso 1, para todos los escenarios (Tabla 5.41). 
Tabla 5.41 Cálculo del indicador HP

\begin{tabular}{|c|c|c|c|}
\hline Indicador & $\begin{array}{c}\text { Sectorización } \\
\text { inicial }\end{array}$ & $\begin{array}{c}\text { Sectorización } \\
\text { energética }\end{array}$ & $\begin{array}{c}\text { Red } \\
\text { Sensorizada }\end{array}$ \\
\hline HP & 1,10 & 1,31 & 1,03 \\
\hline
\end{tabular}

Para abordar la confiabilidad de las redes, se ha analizado el comportamiento de las redes bajo condiciones de incendio (Tabla 5.42).

Tabla 5.42 Cálculo del IPI

\begin{tabular}{|c|c|c|c|}
\hline Indicador & $\begin{array}{c}\text { Sectorización } \\
\text { inicial }\end{array}$ & $\begin{array}{c}\text { Sectorización } \\
\text { energética }\end{array}$ & $\begin{array}{c}\text { Red } \\
\text { Sensorizada }\end{array}$ \\
\hline IPI & 22 & 82 & 16 \\
\hline $\begin{array}{c}\text { № de escenarios de incendio } \\
\text { con incumplimientos }\end{array}$ & $\begin{array}{c}18 \\
3,31 \%\end{array}$ & $\begin{array}{c}44 \\
8,10 \%\end{array}$ & $\begin{array}{c}16 \\
2,90 \%\end{array}$ \\
\hline
\end{tabular}

En la Tabla 5.43 se recogen los valores para los índices de fiabilidad y robustez, así como sus desviaciones respecto a la red sin sectorizar.

Tabla 5.43 Cálculo de indicadores energéticos

\begin{tabular}{|c|c|c|c|}
\hline Indicador & $\begin{array}{c}\text { Sectorización } \\
\text { inicial }\end{array}$ & $\begin{array}{c}\text { Sectorización } \\
\text { energética }\end{array}$ & $\begin{array}{c}\text { Red } \\
\text { Sensorizada }\end{array}$ \\
\hline Índice de fiabilidad & 0,71 & 0,42 & 0,93 \\
\hline Desviación del índice de fiabilidad & $-23,66 \%$ & $-54,84 \%$ & $0 \%$ \\
\hline Índice de la robustez de la red & 0,18 & 0,12 & 0,18 \\
\hline Desviación del índice de robustez & $0 \%$ & $-33,3 \%$ & $0 \%$ \\
\hline
\end{tabular}

Los últimos indicadores por calcular en este apartado corresponderán con la energía entrega a los usuarios, la energía disipada en la fricción en el transporte de esa agua y la eficiencia energética dinámica (Tabla 5.44).

Tabla 5.44 Cálculo de indicadores energéticos

\begin{tabular}{|c|c|c|c|}
\hline Indicador & $\begin{array}{c}\text { Sectorización } \\
\text { inicial }\end{array}$ & $\begin{array}{c}\text { Sectorización } \\
\text { energética }\end{array}$ & $\begin{array}{c}\text { Red } \\
\text { Sensorizada }\end{array}$ \\
\hline Energía entregada a los usuarios $(\mathrm{kWh} / \mathrm{h})$ & 4,34 & 3,38 & 5,08 \\
\hline Energía perdida por fricción en tuberías $(\mathrm{kWh} / \mathrm{h})$ & 0,84 & 1,62 & 0,22 \\
\hline Eficiencia energética dinámica $(\%)$ & 53,21 & 66,00 & 46,38 \\
\hline
\end{tabular}

\subsubsection{Calidad del agua de la red resultante}

En la Tabla 5.45 se recogen los tiempos de permanencia medios del agua en las tuberías. 
Tabla 5.45 Tiempo de permanencia media del agua en tuberías

\begin{tabular}{|c|c|c|c|}
\hline Indicador & $\begin{array}{c}\text { Sectorización } \\
\text { inicial }\end{array}$ & $\begin{array}{c}\text { Sectorización } \\
\text { energética }\end{array}$ & $\begin{array}{c}\text { Red } \\
\text { Sensorizada }\end{array}$ \\
\hline Tiempo de permanencia media $(\mathrm{h})$ & 8,01 & 8,98 & 7,61 \\
\hline
\end{tabular}

Las concentraciones medias y mínimas en los puntos de consumo se muestran en la Tabla 5.46, partiendo de una dosificación constante de 0,9 ppm en el depósito.

Tabla 5.46 Niveles de concentración de cloro en los puntos de consumo

\begin{tabular}{|c|c|c|c|}
\hline $\begin{array}{c}\text { Concentración de cloro en } \\
\text { punto de consumo }\end{array}$ & $\begin{array}{c}\text { Sectorización } \\
\text { inicial }\end{array}$ & $\begin{array}{c}\text { Sectorización } \\
\text { energética }\end{array}$ & $\begin{array}{c}\text { Red } \\
\text { Sensorizada }\end{array}$ \\
\hline Concentración media (ppm) & 0,81 & 0,79 & 0,87 \\
\hline Concentración mínima (ppm) & 0,50 & 0,46 & 0,56 \\
\hline
\end{tabular}

\subsubsection{Coste de implantación}

Para la sectorización actual de la red, es necesario la instalación de dos contadores generales con equipos de comunicación, 1 válvula reductora y 14 válvulas de corte (Tabla 5.47).

Tabla 5.47 Costes directos para la sectorización actual

\begin{tabular}{|l|c|c|c|}
\hline Equipos & Ud. & $\begin{array}{c}\text { Coste } \\
\text { unitario (€) }\end{array}$ & $\begin{array}{c}\text { Coste total } \\
(\boldsymbol{\epsilon})\end{array}$ \\
\hline Contadores sectoriales sobre tubería DN150 & 1 & 3.750 & 3.750 \\
\hline Contadores sectoriales sobre tubería DN90 & 1 & 2.150 & 2.150 \\
\hline Válvulas de corte DN160 & 1 & 2.300 & 2.300 \\
\hline Válvulas de corte DN80 & 5 & 730 & 3.650 \\
\hline Válvulas de corte DN100 & 8 & 1.100 & 8.800 \\
\hline Equipos de comunicación GPRS (Q+P) & 2 & 1.300 & 2.600 \\
\hline Válvula reductora presión DN150 & 1 & 4.750 & 4.750 \\
\hline TOTAL & & & $\mathbf{2 8 . 0 0 0}$ \\
\hline
\end{tabular}

En el caso de la sectorización de la red siguiendo criterios energéticos será necesario instalar 4 contadores sectoriales, 1 válvulas reductoras y 22 válvulas de corte y los correspondientes equipos de telegestión. Siendo así el coste total de implantación de $51.660 €$, según el detalle de la Tabla 5.48.

Tabla 5.48 Costes directos para sectorizar la red según criterios energéticos

\begin{tabular}{|l|c|c|c|}
\hline Equipos & Ud. & $\begin{array}{c}\text { Coste } \\
\text { unitario (€) }\end{array}$ & $\begin{array}{c}\text { Coste } \\
\text { total }(\boldsymbol{\epsilon})\end{array}$ \\
\hline Contadores sectoriales sobre tubería DN150 & 3 & 3.750 & 11.250 \\
\hline Contadores sectoriales sobre tubería DN90 & 2 & 2.150 & 4.300 \\
\hline Válvulas de corte DN50 & 3 & 600 & 1.800 \\
\hline Válvulas de corte DN80 & 7 & 730 & 5.110 \\
\hline Válvulas de corte DN100 & 12 & 1.100 & 13.200 \\
\hline
\end{tabular}




\begin{tabular}{|l|c|c|c|} 
Equipos de comunicación GPRS $(\mathrm{Q}+\mathrm{P})$ & 5 & 1.300 & 6.500 \\
\hline Válvula reductora presión DN150 & 2 & 4.750 & 9.500 \\
\hline TOTAL & & & $\mathbf{5 1 . 6 6 0}$ \\
\hline
\end{tabular}

En el caso de sensorizar la red se optó por instalar 20 sensores de presión, ningún caudalímetro y los correspondientes equipos de telegestión. En este caso, el coste total de implantación necesario asciende a 44.000€ (Tabla 5.49).

Tabla 5.49 Costes directos para sensorizar

\begin{tabular}{|l|c|c|c|}
\hline Equipos & Ud. & $\begin{array}{c}\text { Coste } \\
\text { unitario }(\boldsymbol{(})\end{array}$ & $\begin{array}{c}\text { Coste total } \\
(\boldsymbol{(})\end{array}$ \\
\hline Sensor de presión & 20 & 1.100 & 22.000 \\
\hline Equipos de comunicación GPRS (P) & 20 & 1.100 & 22.000 \\
\hline TOTAL & & & $\mathbf{4 4 . 0 0 0}$ \\
\hline
\end{tabular}

No se consideran, para ninguna de las dos alternativas, otros costes.

\subsubsection{Valoración de las alternativas}

Una vez analizadas todas las métricas, se deberán transformar dichos valores absolutos a valores relativos siguiendo los rangos establecidos en la Tabla 5.4. En la Tabla 5.50 se muestran dichos valores.

Tabla 5.50 Métricas de evaluación y valores de referencia

\begin{tabular}{|c|c|c|c|c|c|c|c|}
\hline \multirow{2}{*}{ Criterios } & \multirow{2}{*}{ Métricas } & \multicolumn{2}{|c|}{ Red actual } & \multicolumn{2}{|c|}{ Sectorización } & \multicolumn{2}{|c|}{ Sensorización } \\
\hline & & $\begin{array}{c}\text { Valor } \\
\text { absoluto }\end{array}$ & $\begin{array}{l}\text { Valor } \\
\text { relativo }\end{array}$ & $\begin{array}{c}\text { Valor } \\
\text { absoluto }\end{array}$ & $\begin{array}{l}\text { Valor } \\
\text { relativo }\end{array}$ & $\begin{array}{c}\text { Valor } \\
\text { absoluto }\end{array}$ & $\begin{array}{l}\text { Valor } \\
\text { relativo }\end{array}$ \\
\hline 1.1 & $\begin{array}{l}\text { Reducción en el coste } \\
\text { anual }\end{array}$ & 0 & 0 & $5,2 \%$ & 1,72 & 0 & 0 \\
\hline 1.2 & Velocidad de inspección & 300 & 3,33 & 300 & 3,33 & 300 & 3,33 \\
\hline \multirow{4}{*}{2.1} & $\begin{array}{l}\text { Variabilidad de la presión } \\
\text { media }\end{array}$ & 26,50 & 5,58 & 18,04 & 6,99 & 35,31 & 4,12 \\
\hline & Exceso de presión media & 27,10 & 2,15 & 17,93 & 4,02 & 33,93 & 1,01 \\
\hline & Déficit de presión media & 0,00 & 10 & 0,00 & 10 & 0,00 & 10 \\
\hline & $\mathrm{HP}$ & 1,10 & 3,67 & 1,31 & 4,37 & 1,03 & 3,43 \\
\hline \multirow{3}{*}{2.2} & IPI & 3,31 & 7,79 & 8,10 & 4,6 & 2,90 & 8,07 \\
\hline & Índice de fiabilidad & 0,71 & 7,16 & 0,42 & 4,24 & 0,93 & 9,31 \\
\hline & Índice de la robustez & 0,18 & 1,82 & 0,12 & 1,21 & 0,29 & 2,93 \\
\hline \multirow{2}{*}{2.3} & $\begin{array}{c}\text { Exceso de Energía } \\
\text { entregada a los usuarios }\end{array}$ & $84 \%$ & 6,09 & $43 \%$ & 7,94 & $115 \%$ & 4,78 \\
\hline & $\begin{array}{c}\text { Eficiencia energética } \\
\text { dinámica }\end{array}$ & 53,21 & 6,88 & 66,00 & 7,73 & 46,38 & 6,43 \\
\hline \multirow{2}{*}{3.1} & $\begin{array}{l}\text { Tiempo máximo de } \\
\text { permanencia }\end{array}$ & 24,00 & 0 & 24,00 & 0 & 24,00 & 0 \\
\hline & $\begin{array}{l}\text { Tiempo medio de } \\
\text { permanencia }\end{array}$ & 8,01 & 6,66 & 8,98 & 6,26 & 7,61 & 6,83 \\
\hline 3.2 & $\begin{array}{c}\text { Concentración media de } \\
\text { cloro }\end{array}$ & 0,81 & 7,89 & 0,79 & 7,67 & 0,87 & 8,56 \\
\hline
\end{tabular}




\begin{tabular}{|c|c|c|c|c|c|c|c|}
\hline \multirow{2}{*}{ Criterios } & Métricas & \multicolumn{2}{|c|}{ Red actual } & \multicolumn{2}{c|}{ Sectorización } & \multicolumn{2}{c|}{ Sensorización } \\
\cline { 2 - 8 } & $\begin{array}{c}\text { Valor } \\
\text { absoluto }\end{array}$ & $\begin{array}{c}\text { Valor } \\
\text { relativo }\end{array}$ & $\begin{array}{c}\text { Valor } \\
\text { absoluto }\end{array}$ & $\begin{array}{c}\text { Valor } \\
\text { relativo }\end{array}$ & $\begin{array}{c}\text { Valor } \\
\text { absoluto }\end{array}$ & $\begin{array}{c}\text { Valor } \\
\text { relativo }\end{array}$ \\
\cline { 2 - 9 } & $\begin{array}{c}\text { Concentración mínima de } \\
\text { cloro }\end{array}$ & 0,50 & 4,44 & 0,46 & 4 & 0,56 & 5,11 \\
\hline 4.1 & $\begin{array}{c}\text { Costes de implantación } \\
\text { directos }\end{array}$ & 933,33 & 7,93 & $1.722,00$ & 6,17 & $1.466,67$ & 6,74 \\
\hline 4.2 & Otros costes & 0,00 & 10 & 0,00 & 10 & 0,00 & 10 \\
\hline
\end{tabular}

Multiplicando dichos valores relativos por su peso asociado (Tabla 5.3) se obtendrá la valoración de cada uno de los criterios, objetivos y la evaluación final entre las dos técnicas objeto de este estudio (Tabla 5.51 y Tabla 5.52).

Tabla 5.51 Evaluación de los objetivos y criterios

\begin{tabular}{|c|c|c|c|c|c|c|c|}
\hline \multirow{4}{*}{$\begin{array}{l}\text { Objetivos } \\
\text { Objetivo } 1\end{array}$} & \multirow{3}{*}{$\begin{array}{l}\text { Criterios de } \\
\text { evaluación }\end{array}$} & \multicolumn{6}{|c|}{ Valoración } \\
\hline & & \multicolumn{2}{|c|}{ Sectorización } & \multicolumn{2}{|c|}{ Sectorización } & \multicolumn{2}{|c|}{ Sensorización } \\
\hline & & 0,00 & & 1,72 & & 0,00 & \\
\hline & Criterio 1.2 & 3,33 & & 3,33 & & 3,33 & \\
\hline \multirow{3}{*}{ Objetivo 2} & Criterio 2.1 & 5,59 & \multirow{3}{*}{5,74} & 6,57 & \multirow{3}{*}{5,74} & 4,93 & \multirow{3}{*}{5,68} \\
\hline & Criterio 2.2 & 5,39 & & 3,24 & & 6,65 & \\
\hline & Criterio 2.3 & 6,54 & & 7,82 & & 5,72 & \\
\hline \multirow{2}{*}{ Objetivo 3} & Criterio 3.1 & 2,22 & \multirow{2}{*}{4,62} & 2,09 & \multirow{2}{*}{4,34} & 2,28 & \multirow{2}{*}{5,09} \\
\hline & Criterio 3.2 & 5,82 & & 5,47 & & 6,49 & \\
\hline \multirow{2}{*}{ Objetivo 4} & Criterio 4.1 & 7,93 & \multirow{2}{*}{8,62} & 6,17 & \multirow{2}{*}{7,45} & 6,74 & \multirow{2}{*}{7,83} \\
\hline & Criterio 4.2 & 10,00 & & 10,00 & & 10,00 & \\
\hline
\end{tabular}

Tabla 5.52 Evaluación final entre las dos técnicas y el escenario inicial

\begin{tabular}{|c|c|}
\hline Técnica & $\begin{array}{c}\text { Valoración } \\
\text { Final }\end{array}$ \\
\hline Sectorización inicial & 4,50 \\
\hline Sectorización según criterios energéticos & 4,56 \\
\hline Sensorización & 4,47 \\
\hline
\end{tabular}

\subsubsection{Discusión de los resultados obtenidos}

En el segundo caso de estudio, a diferencia del primero, se ha partido de una red de distribución ya sectorizada, según criterios puramente prácticos, y con una gestión de presión sobre la misma. Este escenario se ha comparado con una sectorización según criterios energéticos acompañada de una gestión de presiones y con el uso de sensores de presión y caudal sobre la red sin dividir para la localización de volúmenes incontrolados.

Analizando la valoración global (Tabla 5.52) se podría afirmar que las tres técnicas tienen una valoración similar, con la sectorización según criterios energéticos destacando ligeramente. Aunque el número de objetivos y criterios seleccionados son adecuados, reside en la alta 
cantidad de métricas elegidas esta valoración tan pareja entre las técnicas, laminando un comportamiento medio frente a desviaciones de alguna de las métricas.

Desglosando la evaluación final, se pueden observar unos resultados en línea con lo esperado a priori con los objetivos 2,3 y 4, pero no siendo así para el objetivo 1.

Respecto al primer objetivo, efectividad y eficiencia en la búsqueda de fugas no declaradas, a priori se esperaba una mejora importante en los dos escenarios, pero el aporte ha sido mínimo. Según la política de inspección seguida en este caso, la frecuencia de intervención depende de la longitud total de la red, independientemente de que se encuentre sectorizada o no, y del volumen fugado anualmente. Ha sido en la sectorización según criterios energéticos dónde se ha conseguida reducir la presión media respecto la sectorización actual y con ello menores volúmenes fugados. En el objetivo 2, capacidad hidráulica de la red resultante, los resultados han ido en la línea de lo esperado. Por un lado, el sectorizar la red ha mejorado el nivel de presiones y, con ello, la eficiencia energética dinámica. Pero por otro, se ha visto reducida la robustez del sistema. Desde el punto de vista de la calidad del agua, objetivo 3, sectorizar la red afecta negativamente a los parámetros analizados, haciendo que el agua permanezca más tiempo en la red y que, con ello, el nivel de cloro se vea mermado. Respecto al objetivo 4, Costes de implantación, la sectorización inicial implica un menor coste de inversión frente a la sectorización siguiendo criterios energéticos o la red sensorizada planteada, pero este comportamiento no se puede generalizar ni vincular a una metodología u otra. Los costes de implantación dependerán de la topología de la red y el punto de partida de las infraestructuras.

Entrando en detalle en el objetivo 1, para los tres escenarios analizados hay que destacar que la política de búsqueda seguida es independiente de la topología de la red, y que la frecuencia de intervención depende de la longitud total de todo el sistema y el volumen anual fugado. Esto hace que no se consiga una mejora significativa en la reducción de costes. Sí que se aprecia un pequeño aumento, del $5,43 \%$, en el caso de la sectorización siguiente criterios energéticos. Y con ello, un menor coste anual por el volumen fugado y los trabajos de búsqueda de fugas, que se reducen en un 5,2\%. Esto es debido a que esta disposición de los sectores consigue unos niveles de presiones más bajos que los otros dos escenarios. Respecto a la velocidad de inspección de la red es la misma en los tres escenarios y se mantiene en el nivel inicial planteado, $300 \mathrm{~m} / \mathrm{h}$. La política de intervención marca que las inspecciones se tienen que hacer a toda la red, y no a sectores o zonas concretas, de manera periódica. Por lo tanto, la división de la red en zonas aisladas más pequeñas o el incremento en el número de sensores con el objetivo de acotar con mayor precisión la zona donde se está produciendo el volumen incontrolado es independiente de la política de intervención seguida en este caso. Así, la nota de este objetivo es muy baja, situándose en 1,43 para la sectorización actual y para la red sensorizada y 2,41 para la sectorización según criterios energéticos.

Sobre el objetivo 2, se observa que los dos escenarios sectorizados presentan una misma valoración, 5,74, superior a los 5,68 de la red sensorizada.

Desgranando estos valores, se observan grandes diferencias entre los escenarios donde se ha sectorizado la red y se implantado una gestión de presión y la red totalmente abierta con sensores y sin gestión de presiones, como era de esperar. La red con sensórica presenta los niveles más altos de presión, con una presión media que se sitúa en los 53,93 mca y unos valores máximos y mínimos de 74,97 y 32,20 mca, respectivamente. En el caso de la 
sectorización inicial, se mantiene un valor máximo de presión de 75,10 mca, pero descae los valores medios y mínimos a 44,50 y 21,16 mca, respectivamente. Este descenso viene impuesto por las pérdidas de carga introducidas en el sistema, que aumentan en un $281 \%$ respecto a la red sin sectorizar. Los resultados para la sectorización según criterios energéticos son algo mejores, ya que el valor máximo de presión cae hasta los 49,58 mca y a 37,11 mca la presión media. La presión mínima se sitúa en los 21,20 mca. Este comportamiento de los niveles de presiones va en línea con el comportamiento del exceso de presión media y de los valores de las desviaciones de esta para los tres escenarios. Encontramos valores más bajos para la sectorización según criterios energéticos y más altos para la red sensorizada. La sectorización inicial presenta unos valores intermedios, pero más cercanos a la red sensorizada. Siguiendo en esta línea, la red sectorizada según criterios energéticos presenta un nivel general de las presiones con un valor de HP de 1,31, frente a los 1,10 de la sectorización inicial o los 1,03 que presenta la red sin sectorizar. Es decir, unos valores más adecuados de presiones en la red. Así, se puede afirmar que es la sectorización según criterios energéticos quién presenta un mejor comportamiento en el criterio 2.1, Presión hidráulica en red, con una valoración de 6,57 frente a 5,59 de la sectorización inicial o 4,93 de la red sensorizada.

Abordando la resiliencia de los escenarios, se obtienen unos índices de robustez similares entre la sectorización inicial y la red sensorizada, y algo mayores en el caso de la fiabilidad para la red sensorizada. En cambio, y debido al cierre de mallas, en la sectorización de la red según criterios energéticos se obtiene una disminución del índice de robustez de un 33,3\% y en un $54,84 \%$ el índice de fiabilidad. Por lo que, la red inicial sin alterar presenta unos valores más altos de exceso de energía disponible, una distribución de tuberías más uniformes y, por lo tanto, mayores capacidades de recuperación de la red. Este efecto se ve con mayor claridad al analizar las redes bajo condiciones de incendio. Simulando un incendio de $8,33 \mathrm{l} / \mathrm{s}$ en todos los escenarios, y en todos los puntos posibles, se obtiene que en el $8,1 \%$ de los casos para la red sectorizada según criterios energéticos no se estará cumpliendo con las condiciones impuestas por la normativa. Este valor desciende hasta el 3,31\% para la sectorización inicial y $2,9 \%$ para la red sensorizada. En este caso, es la red sensorizada la que presenta un mejor comportamiento bajo en criterio 2.2, Resiliencia de la red, con una valoración 6,65 frente a 5,39 de la sectorización inicial y 3,24 de la red sectorizada según criterios energéticos.

Analizando los indicadores energéticos, se observa una tendencia en línea con el comportamiento de los niveles de presiones. La red sectorizada siguiendo criterios energéticos presenta unos niveles de energía entregada a los usuarios un 33,47\% más bajos respecto a la red sin sectorizada y una eficiencia energética dinámica del $66 \%$. En el caso de la sectorización inicial, los niveles de energía entregada a los usuarios se sitúan en un $14,57 \%$ por debajo, situándose su eficiencia energética dinámica en 53,21\%. La red sensorizada, sin sectorizar, presenta los valores más bajos de este indicador, con un 46,38\%. Con todo ello, es la sectorización según criterios energéticos quién presenta un mejor comportamiento en el criterio 2.3, Energía disponible en red, con una valoración de 7,82 frente a 6,54 de la sectorización inicial o 5,72 de la red sensorizada.

Desgranando el objetivo 3, calidad del agua de la red resultante, los resultados analizados están en línea con los esperados. Los mejores comportamientos se encuentran en la red sensorizada, debido a que cuenta con todas las tuberías abiertas. Así, se obtiene para dicho escenario una valoración de 5,09 frente a la sectorización actual con un 4,62 y un 4,34 de la sectorización según criterios energéticos. En la red sensorizada, sobre la cual no se realiza ningún cierre de tubería, el tiempo medio de permanencia del agua en el sistema se sitúa en 
7,61 horas. Para la sectorización inicial con 14 cortes, este tiempo aumenta en un 5,26\%, hasta 8,01 horas, y en un $15,26 \%$ en la sectorización según criterios energéticos, hasta el valor de 8,98 horas, debido a las 22 de válvulas de corte a instalar. Los niveles de concentración medios y mínimos de cloro en red se mantienen prácticamente iguales para los tres escenarios, aunque ligeramente superiores para el caso de la red sensorizada.

El último factor analizado ha sido el aspecto económico. Los tres escenarios requieren de ciertas inversiones para poder ejecutarse. En este caso, ha sido la sectorización inicial la que requiere de un mayor capital, seguida de la red sensorizada y, en último lugar, la sectorización según criterios energéticos. En los tres escenarios, las valoraciones de este objetivo han sido altas, 8,62 para la sectorización inicial, 7,83 la sensorización de la red y 7,45 la sectorización según criterios energéticos. Ahora bien, este resultado no se puede generalizar ni extrapolar a otro caso ya que el resultado final dependerá de la situación particular de cada caso, así como de la situación presupuestaria disponible. 


\section{Capítulo 6 Conclusiones}

\subsection{Introducción}

El reto al que nos vemos obligados a enfrentarnos es complicado y apasionante a partes iguales. El planeta se encuentra sumido en una emergencia medioambiental ligada al cambio climático, la población mundial está creciendo a un ritmo nunca visto, frente a una capacidad de producción del planeta cada vez más mermada por la sobre explotación de los recursos, y la concentración de la población en ciudades y la urbanización de estas crece a un ritmo acelerado.

Todo ello, nos obliga a actuar bajo políticas de sostenibilidad y de optimización de recursos, buscando en todo momento la eficiencia en los procesos. En el caso que nos ocupa, los gestores de los sistemas de distribución de agua tienen la obligación de efectuar un uso eficiente del agua y optimizar el uso de la energía. $Y$ hacerlo siempre, además, bajo el paradigma de un servicio continuo, seguro y de calidad. Para todo ello y de manera generalizada, cuentan con unas infraestructuras deficientes (las más de las veces) y unas tarifas insuficientes para mantener los servicios. Así, la única vía aceptable para conseguir los objetivos marcados pasa por la realización de acciones transversales enfocadas a la optimización de los procesos y el ahorro de recursos.

Con este objeto, una de las líneas de mejora recae sobre uno de los principales problemas de los gestores de los sistemas de distribución de agua: las fugas de agua. Estas representan un problema económico, ambiental, de sostenibilidad y potencialmente de salud y seguridad, cuya reducción presenta un reto y una obligación para los gestores. En esta línea, es necesario actuar de manera global con estrategias reales, claras y alcanzables. Cualquier gestor debe ser capaz de entenderlas y replicarlas sin mayor dificultad. 
Para poder introducir al gestor y su problemática en la línea de investigación, la presente tesis ha sido desarrollada en el seno de la empresa Aguas de Valencia S.A., optando así a la mención de Doctorado Industrial por la Universitat Politècnica de València. Con ello, se ha conseguido un marco de colaboración entre la Universitat Politècnica de València y la empresa privada que, además de la presente tesis doctoral, ha contribuido a generar una fenomenal simbiosis. Se ha permitido introducir en todos los desarrollos teóricos las limitaciones impuestas por los condicionantes propios de las redes reales. Como resultado, se han obtenido unas herramientas cuya sencillez permite su implantación entre los gestores de redes de abastecimiento.

En lo que sigue, se definen las principales conclusiones de la tesis doctoral agrupadas según los tres objetivos que ha guiado el trabajo de investigación: se ha descrito un método de sectorización según criterios energéticos que facilite la clara delimitación de cada uno de los sectores hidrométricos de la red y que facilite la implantación u operativa de otras técnicas de gestión (apartado 6.2), se ha propuesto el uso de sensores para la adecuada localización de las fugas en la red como técnica alternativa a la sectorización (apartado 6.3) y se han comparado ambas técnicas (apartado 6.4). Por último, se muestran los desarrollos futuros planteados (apartado 6.5).

\subsection{Criterios energéticos como guía en el diseño de la sectorización}

Ya que la erradicación completa de los volúmenes incontrolados fugados es, cuanto menos, complicada, todos los trabajos se centran en reducir el tiempo en el que una fuga pueda estar activa. Para conseguir este objetivo, la sectorización de las redes ha demostrado ser una metodología eficaz.

No existen directrices claras para la definición de los sectores, sino que la comunidad científica cuenta con multitud de recomendaciones, basadas en la experiencia de otros diseñadores. Además, es posible encontrar recomendaciones muy dispares o con poco fundamento técnico para una misma variable de decisión. Esto obliga al diseñador a continuar con diseños basados en su experiencia, procesos manuales y de prueba y error.

Este trabajo presenta una metodología sistemática para el diseño de la sectorización de una red de distribución de agua atendiendo a criterios energéticos. Este método se apoya en la topología de la red y en el comportamiento del sistema de la red.

Así, se delimitan claramente los sectores hidrométricos organizados según niveles homogéneos de eficiencia dinámica energética. Por tanto, se facilita el proceso de diseño y se favorecen posteriores procesos de gestión de la presión (objetivo específico 1.3). Es decir, se consiguen los beneficios propios de esta técnica, pero además se obtienen otras mejoras indirectas ligadas al consumo energético.

Con dicha metodología se ha conseguido un doble objetivo. En primer lugar, la metodología presentada aporta nuevos criterios para la delimitación de la sectorización (objetivo específico 1.1.) que buscan la optimización del funcionamiento hidráulico de la red. La metodología propone de manera autónoma (objetivo específico 1.2) la delimitación precisa de los sectores sin considerar la experiencia del diseñador ni la topología de la red de distribución de agua a 
presión. En segundo lugar, la nueva disposición de la red permite la adecuada gestión de la presión para la reducción de las fugas.

Se puede comprobar como en todos los casos analizados durante este trabajo (apartados 3.3.3.1, 3.3.4.1, 5.3.1 y 5.3.2) la sectorización ha conseguido unos menores niveles medios de presión y, en consecuencia, mejores valores de rendimiento energéticos dinámico. En el caso 2 del Capítulo 5 (5.3.2) se compara una sectorización real, ejecutada según criterios tradicionales, con la resultante de esta metodología. Ambas con gestión de la presión. Como resultado, la sectorización según criterios energéticos consigue reducir en un 16,61\% la presión media, de 44,50 mca a 37,11 mca, y en un 33,98\% la presión máxima, de 75,10 mca a 49,58 mca. Respecto al rendimiento energético dinámico, esta metodología incrementa en un $24,04 \%$ su valor, pasando del $53,21 \%$ al $66 \%$.

Así, se puede concluir que esta tesis aporta de manera clara al ámbito del conocimiento una metodología para la definición de los sectores de una red de distribución de agua bajo criterios energéticos, sin necesidad del aporte del diseñador y que favorece posteriores procesos de gestión de la presión.

\subsection{Sensorización como herramienta para la localización de las fugas}

La sectorización ha resultado ser una técnica eficaz en la búsqueda de fugas, aunque, en contrapartida, supone una pérdida de capacidad hidráulica y una merma en la calidad del agua. Por ello, y respondiendo al objetivo 2, se ha desarrollado una metodología alternativa para la localización de las fugas.

La metodología en sí misma está estructurada en dos partes. En la primera parte, se plantea un análisis de sensibilidad cuando se produce un ligero aumento de la demanda y se analizan las variaciones de los caudales circulantes y de las alturas piezométricas en los nudos. Como resultado se obtiene la variación promedio de los caudales en las líneas y de las presiones en los nudos ante cualquier incremento de consumo en la red de magnitud dada (objetivo específico 2.1). La segunda parte resuelve las ecuaciones de conservación de masa y energía, considerando como incógnitas las demandas de agua desconocidas en cada nudo, debido a las fugas, y los caudales circulantes por las tuberías, y como variables conocidas las alturas piezométricas en los nudos, gracias al análisis de sensibilidad (objetivo específico 2.2). Las mediciones registradas por los sensores de presión y flujo se consideran condiciones de contorno del problema.

Resta solo destacar dos apuntes adicionales. El primero es la versatilidad del método: si bien, todo el trabajo se enfoca desde la perspectiva de la localización de fugas, los conceptos utilizados en la formulación matemática permiten que sea igualmente aplicable a la localización de consumos clandestinos. El segundo, es que esta metodología destaca por su rapidez de cálculo en la resolución del escenario a abordar, lo que debido a la gran combinatoria posible en la consideración de fugas a localizar, será una de sus principales ventajas frente a estudios similares.

Analizando el caso de estudio 1 (apartado 5.3.1), ante una fuga de $30 \mathrm{l} / \mathrm{min}$, la longitud de búsqueda promedio se reduce de $50 \mathrm{~km}$ a 6,58 km usando 15 sensores de presión colocados 
de manera homogénea por toda la red. En el caso de estudio 2 (apartado 5.3.2), para una fuga del mismo tamaño y el uso de 20 sensores de presión, la longitud promedio de búsqueda pasa de $30,8 \mathrm{~km}$ a $8,38 \mathrm{~km}$. Incremento el número de equipos, hasta los 40 sensores, esta longitud se ve reducida hasta los $5,85 \mathrm{~km}$.

En este caso, la tesis aporta una nueva metodología eficaz para la localización de las fugas basada en la monitorización de las redes de distribución de agua que puede sustituir la necesidad de sectorizar la misma.

\subsection{Comparación de las metodologías}

Con el fin último de minimizar el problema de los volúmenes fugados incontrolados, a lo largo de este trabajo se han desarrollado dos alternativas eficaces. Para analizar la bondad global de las alternativas (objetivo 3), se ha planteado un sistema basado en la evaluación de los objetivos marcados según criterios de evaluación y métricas. Sistema que destaca por su sencillez a la hora de su aplicación. Con este sistema, se evalúa con una escala común cada alternativa.

En el caso 1 del Capítulo 5 (apartado 5.3.1), la técnica de la sensorización de la red obtiene una valoración superior a la obtenida por la sectorización según criterios energéticos, 6,98 frente a 6,38, respectivamente. Pero en el caso 2 (apartado 5.3.2), es la técnica de la sectorización la que obtiene una valoración ligeramente superior. Por ello, se puede afirmar que no existe una solución óptima sobre otra para todos los sistemas de manera global. Esto dependerá de los objetivos a conseguir marcados por el gestor, así como la política operativa en cada caso. Evidentemente, si en lugar de analizar el impacto global de las técnicas se analiza el impacto por criterios, sí se vislumbra qué técnica es más aconsejable.

Sobre los trabajos de búsqueda de fuga, ambas técnicas dan como resultado una delimitación de la zona donde se está produciendo la fuga. En el caso de la sectorización, esta delimitación corresponde a cada uno de los sectores. Y en el caso de la sensorización, la longitud de búsqueda promedio dependerá de cada caso, del número de sensores instalaciones y la ubicación de estos. Para el caso 1, la longitud promedio de los sectores es de 12,8 km frente a los $6,58 \mathrm{~km}$ de la longitud de búsqueda promedio de la red sensorizada. En el caso 2, el sector medio tiene una longitud de $6,18 \mathrm{~km}$ y la longitud de búsqueda promedio está entre los 5,85 y 8,38 km según el número de sensores a instalar. La influencia de ambas técnicas sobre los trabajos de correlación de la red dependerá de la política seguida, llegando a ser nula como en el caso 2.

Analizando el comportamiento hidráulico, la sectorización según criterios energéticos presenta mejores resultados sobre los niveles de presión y energía disponible en red, consiguiendo menores niveles medios. En cambio, el uso de la sensorización dota de mayor robustez a la red porque no supone una disminución de la capacidad hidráulica.

Respecto a la calidad del agua, la sensorización resulta ser una mejor opción al no obligar el cierre de tramos. Con ello está permitiendo una mejor circulación del agua por la red, obtenido mejores indicadores de tiempo de permanencia del agua (tiempos menores) en la red o de los niveles de cloro (el decaimiento del cloro es menor por ser el tiempo de permanencia menor).

Ambas técnicas requieren de inversión. Pero la dotación económica de cada método es distinta para cada caso, es decir, no siempre un método demanda más inversión. En el caso 
1, la inversión necesaria para sensorizar la red es mayor que la inversión requerida por la sectorización. En cambio, en el caso 2 ocurre lo contrario.

Se puede concluir que no existe una solución óptima sobre las otras, sino que todos los factores del entorno se ven influenciados por el método seleccionado. Su uso no es excluyente, pudiendo emplearse conjuntamente ambas metodologías sobre la misma red para conseguir unos mejores y globales resultados sobre los volúmenes incontrolados.

\subsection{Futuras líneas de trabajo}

En este trabajo se ha planteado una serie de objetivos, los cuales han ido guiando en todo momento la línea de investigación. Pero no todo ha sido resuelto de manera satisfactoria, ya sea bien porque el alcance de todo trabajo es, y debe ser, finito o bien por las propias limitaciones que las infraestructuras han impuesto a los desarrollos teóricos alcanzados. No puede considerarse este hecho como un punto negativo del trabajo, sino como una oportunidad para futuras líneas de investigación.

Así, se destacan las siguientes líneas de trabajo potenciales a desarrollar en el seno de las técnicas orientadas a la mejora continua de la eficiencia hidráulica de los sistemas de distribución de agua.

- Diseño totalmente autónomo de la sectorización de las redes, según criterios hidráulicos y económicos, que garantice el punto de funcionamiento óptimo de la red una vez sectorizada. En el presente trabajo se presenta una metodología sistemática que consigue unas delimitaciones claras de unos sectores organizados según niveles homogéneos de eficiencia dinámica energética. En estos casos, el prediseño tiene que ser validado con un modelo de simulación que verifique que el escenario planteado cumple las condiciones mínimas de presión y caudal a lo largo de toda la red. Además, conviene subrayar que la metodología planteada emplea el escenario de funcionamiento actual, y agrupa los elementos según este escenario. Es decir, se agrupan los elementos según los valores de presión y caudal, en un escenario sin sectorizar. Pero, para sectorizar la red será necesario cerrar tuberías para aislar las diferentes zonas, con lo que el punto de funcionamiento cambia y no se garantiza que la gestión de presión a realizar sea la óptima.

La resolución de este problema no es muy compleja desde el punto de vista heurístico. Simplemente empleando algoritmos genéticos se puede encontrar una solución óptima o cercana a ella. Ahora bien, muchas de las herramientas heurísticas no presentan un desarrollo sencillo y su uso no es trivial. Por ello, es necesario abordar una metodología sencilla y transparente que invite a su implantación de manera directa ente los gestores de los sistemas de distribución de agua. Aquí radica el éxito de la presente metodología, en la sencillez de su implantación.

- Otros criterios de diseño: Otra línea de trabajo debe centrarse en obtener la disposición de sectores que garantice unos niveles máximos de calidad del agua. Para ello, se deberá seguir la misma filosofía que la recogida en este trabajo a la hora de la definición de la sectorización según criterios energéticos. Se deberá analizar cómo organizar los sectores, de tal forma que se maximice la calidad del agua desde su entrada en el sistema hasta que llega al punto de consumo. 
- Combinación de las dos técnicas presentadas: A lo largo de todo el trabajo se ha presentado la sectorización según criterios energéticos como una técnica contra los volúmenes incontrolados fugados. Para suplir las pequeñas desventajas que presenta esta técnica, se ha abordado el uso de sensores como una nueva herramienta para la localización de las fugas. Incluso, se ha realizado una comparativa entre ambas para analizar cuál de las dos sería más recomendable para el gestor. Otra futura línea de trabajo deberá analizar cómo compatibilizar conjuntamente ambas técnicas y qué resultados se obtendrán.

- Aplicar la técnica de la sensorización con dos fugas simultáneas o más: La sensorización de las redes como herramienta para la localización de fugas ha resultado ser una herramienta adecuada para ello. Pero, parte de la hipótesis de que tan solo se está produciendo una fuga en un único punto. Con esta distribución, se analiza la sensibilidad de toda la red y se ubica la zona donde se localiza. Ahora bien, pueden producirse dos o más fugas en un mismo periodo de tiempo. Una última futura línea de trabajo estudiará si es posible ampliar la metodología presentada en el capítulo 4 al caso de dos o más fugas simultáneas, así como obtener la sensibilidad de la red y la posible ubicación de dichas fugas. 


\section{Capítulo 7 Referencias}

ADB. (2008). Data Book of Southeast Asian Water Utilities 2005. Asian Development Bank. Recuperado el 27 de enero de 2021, de http://hdl.handle.net/11540/226

AEAS, \& AGA. (2018). XV Estudio Nacional de Suministro de Agua Potable y Saneamiento en España 2018. Asociación Española de Abastecimientos de Agua y Saneamiento (AEAS) y Asociación Española de Empresas Gestoras de los Servicios de Agua Urbana (AGA). Recuperado el 31 de enero de 2021, de https://www.asoaeas.com/sites/default/files/Documentos/Nota\%20de\%20prensa\%20 XV\%20Estudio\%20de\%20Suministro.pdf

AEAS, \& AGA. (2020). XVI Estudio Nacional de Suministro de Agua Potable y Saneamiento en España 2020. Asociación Española de Abastecimientos de Agua y Saneamiento (AEAS) y Asociación Española de Empresas Gestoras de los Servicios de Agua Urbana (AGA). Recuperado el 31 de enero de 2021, de https://www.asoaeas.com/?q=content/xvi-estudio-nacional-aeas-aga

Alegre, H., \& Coelho, S. (2012). Infrastructure Asset Management of Urban Water Systems. En Water Supply System Analysis (págs. 49-73). Avi Ostfeld. doi:10.5772/52377

Alegre, H., Baptista, J., Cabrera Jr, E., Cubillo, F., Duarte, P., Hirner, W., \& Parena, R. (2016). Performance indicators for water supply services. IWA publishing.

Alegre, H., Hirnir, W., Baptista, J., \& Parena, R. (2000). Performance Indicators for Water Supply Services. London: IWA Publishing.

Alkasseh, J., Adlan, M., Abustan, I., Aziz, H., \& Hanif, A. (2013). Applying Minimum Night Flow to Estimate Water Loss Using Statistical Modeling: A Case Study in Kinta Valley, Malaysia. Water Resources Management, 27(5), 1439-1455. doi:10.1007/s11269-0120247-2 
Almandoz, J., Cabrera, E., Arregui, F., Cabrera, E., \& Cobacho, R. (2005). Leakage Assessment through Water Distribution Network Simulation. Journal of Water Resources Planning and Management, 131(6), 458-466. doi:10.1061/(asce)07339496(2005)131:6(458)

Alvisi, S., \& Franchini, M. (2014). A Procedure for the Design of District Metered Areas in Water Distribution Systems. Procedia Engineering, 70, 41-50. doi:10.1016/j.proeng.2014.02.006

Araujo, L., Ramos, H., \& Coelho, S. (2006). Pressure Control for Leakage Minimisation in Water Distribution Systems Management. Water Resources Management, 20(1), 133149. doi:10.1007/s11269-006-4635-3

Armand, H., Stoianov, I., \& Graham, N. (2015). Investigating the Impact of Sectorized Networks on Discoloration. Procedia Engineering, 119, 407-415. doi:10.1016/j.proeng.2015.08.902

Atkinson, S., Farmani, R., Fayyaz, A., \& Butler, D. (2014). Reliability indicators for water distribution system design: Comparison. J. Water Resour. Plann. Manage, 140(2), 160168. doi:10.1061/(ASCE)WR.1943-5452.0000304

Awad, H., Kapelan, Z., Savic, D., \& Emerson, A. (2009). Optimal setting of time-modulated pressure reducing valves in water distribution networks using genetic algorithms. En Integrating water systems (págs. 31-37). Boxall \& Maksimovic (eds).

Awumah, K., Goulter, I., \& Bhatt, S. K. (1991). Entropy-Based Redundancy Measures in WaterDistribution Networks. Journal of Hydraulic Engineering, 117(5), 595-614. doi:10.1061/(asce)0733-9429(1991)117:5(595)

AWWA. (1990). Manual of water supply practices: Water audits and leak detection (Vol. 1st ed. (M36. ISBN 089867 4850)). Denver, CO: American Water Works Association.

AWWA, \& AWWARF. (1992). Water Industry Database: Utility Profiles. Denver: American Water Works Association and American Water Works Association Research.

Babayan, A., Kapelan, Z., Savic, D., \& Walters, G. (2005). Least-cost design of water distribution networks under demand uncertainty. Journal of Water Resources Planning and Management, 131(5), 375-382. doi:10.1061/(asce)0733-9496(2005)131:5(375)

Baños, R., Reca, J., Martínez, J., Gil, C., \& Márquez, A. L. (2011). Resilience Indexes for Water Distribution Network Design: A Performance Analysis Under Demand Uncertainty. Water Resources Management, 25(10), 2351-2366. doi:10.1007/s11269-011-9812-3

Bartolín Ayala, H. J. (2013). Confección de modelos de redes de distribución de agua desde un Sig y desarrollo de herramientas de apoyo a la toma de decisiones. Doctoral dissertation, Universitat Politècnica de València.

Biggs, E. M., Bruce, E., Boruff, B., Duncan, J. M., Horsley, J., Pauli, N., \& Imanari, Y. (2015). Sustainable development and the water-energy-food nexus: A perspective on livelihoods. Environmental Science \& Policy, 54, 389-397. doi:10.1016/j.envsci.2015.08.002 
Blesa, J., Nejjari, F., \& Sarrate, R. (2016). Robust sensor placement for leak location: analysis and design. Journal of Hydroinformatics, 18(1), 136-148. doi:10.2166/hydro.2015.021.

Boulos, F., \& Aboujaoude, S. (2011). Managing leaks using flow step-testing, network modeling, and field measurement. Journal-American Water Works Association, 103(2), 90-97. doi:10.1002/j.1551-8833.2011.tb11404.x

Boulos, P. F., \& Bros, C. M. (2010). Assessing the carbon footprint of water supply and distribution systems. Journal American Water Works Association, 102(11), 47-54. doi:10.1002/j.1551-8833.2010.tb11338.x

Brentan, B. M., Campbell, E., Meirelles, G. L., Luvizotto, E., \& Izquierdo, J. (2017). Social Network Community Detection for DMA Creation: Criteria Analysis through Multilevel Optimization. Mathematical Problems in Engineering, 1-12. doi:10.1155/2017/9053238

Cabrera Jr., E., \& Pardo, M. A. (2008). Performance Assessment of Urban Infrastructure Services. IWA Publishing.

Cabrera, E. (2018). Indicadores de desempeño para servicios de abastecimiento de agua. Valencia: Universitat Politècnica de València.

Cabrera, E., Pardo, M. A., Cobacho, R., \& Cabrera, E. (2010). Energy Audit of Water Networks. Journal of Water Resources Planning and Management, 136(6), 669-677. doi:10.1061/(asce)wr.1943-5452.0000077

Campbell, E. (2017). Sectorización de Redes de Abastecimiento de Agua Potable basada en detección de comunidades en redes sociales y optimización heurística. Tesis Doctoral. Universitat Politècnica de València - Valencia.

Campbell, E., Izquierdo, J., Montalvo, I., \& Pérez-García, R. (2016). A Novel Water Supply Network Sectorization Methodology Based on a Complete Economic Analysis, Including Uncertainties. Water, 8(5), 179. doi:10.3390/w8050179

Casillas, M. V., Garza-Castañón, L. E., \& Cayuela, V. P. (2014). Model-based leak detection and location in water distribution networks considering an extended-horizon analysis of pressure sensitivities. Journal of Hydroinformatics, 16(3), 649-670. doi:10.2166/hydro.2013.019

Casillas, M. V., Garza-Castañón, L. E., \& Puig, V. (2015). Sensor placement for leak location in water distribution networks using the leak signature space. IFAC-PapersOnLine, 48(21), 214-219. doi:10.1016/j.ifacol.2015.09.530

Castro Gama, M. E., Quan, P., Jonoski, A., \& Chiesa, C. (2014). Model-based Sectorization of water distribution networks for increased energy efficiency. CUNY Academic Works. Obtenido de https://academicworks.cuny.edu/cc_conf_hic/233

Charalambous, B., Foufeas, D., \& Petroulias, N. (2014). Leak detection and water loss management. Water Utility Journal, 8(3), 25-30.

Chatzivasili, S., Papadimitriou, K., \& Kanakoudis, V. (2019). Optimizing the Formation of DMAs in a Water Distribution Network through Advanced Modelling. Water, 11(2), 278. doi:10.3390/w11020278 
Cheng, W., Shen, Y., Zhang, X., Xu, G., Zhu, Z., \& Long, Z. (2020). Research on Hydraulic Safety Assessment of Water Distribution Systems. Water, 12(6), 1733. doi:10.3390/w12061733

Chong, C. Y., \& Kumar, S. P. (2003). Sensor networks: Evolution, opportunities, and challenges. Proceedings of the IEEE, 91(8), 1247-1256. doi:10.1109/jproc.2003.814918

Cobacho, R., Arregui, F., Soriano, J., \& Cabrera, E. (2014). Including leakage in network models: an application to calibrate leak valves in EPANET. Journal of Water Supply: Research and Technology-Aqua, 64(2), 130-138. doi:10.2166/aqua.2014.197

Coelho, B., \& Andrade-Campos, A. (2014). Efficiency achievement in water supply systems A review. Renewable and Sustainable Energy Reviews, 30, 59-84. doi:10.1016/j.rser.2013.09.010

Coelho, S. T. (1997). Performance in water distribution: A systems approach. Taylor \& Francis Group.

Colombo, A. F., \& Karney, B. W. (2002). Energy and Costs of Leaky Pipes: Toward Comprehensive Picture. Journal of Water Resources Planning and Management, 128(6), 441-450. doi:10.1061/(asce)0733-9496(2002)128:6(441)

Colombo, A., \& Karney, B. (2002). Energy and costs of leaky pipes: Toward comprehensive picture. Journal of Water Resources Planning and Management, 128(6), 441- 450. doi:doi.org/10.1061/(ASCE)0733-9496(2002)128:6(441)

Conde, C. P. (1996). Sensores ópticos (Vol. 30). Universitát de València.

Confederación Hidrográfica del Júcar. (2003). Recuperación de costes de los servicios del agua demarcación hidrográfica del Júcar. Ministerio de Agricultura, Alimentación y Medio Ambiente. España. .

Consejería de Agricultura, Pesca y Medio Ambiente. (2009). Recuperación de los costes de los servicios de agua. Junta de Andalucía. Obtenido de https://www.juntadeandalucia.es/medioambiente/portal_web/agencia_andaluza_del_ agua/nueva_organizacion_gestion_integral_agua/planificacion/planes_aprobados_co nsejo_gobierno/dh_mediterraneo_aprobado/Anejos_memoria/Anejo_IX/ANEJO_IX.p df

Cross, H. (1936). Analysis of flow in networks of conduits or conductors. Engineering Experiment Station, University oflllions Bulletin, 286.

Dandy, G., Roberts, A., Hewitson, C., \& Chrystie, P. (2008). Sustainability Objectives For The Optimization Of Water Distribution Networks. Water Distribution Systems Analysis Symposium 2006, 1-11. doi:10.1061/40941(247)83

de Nicolás, V. L., Laguna-Peñuelas, F., \& Vidueira, P. (2014). Criterio para la optimización energética de redes ramificadas de agua. Tecnología y ciencias del agua, 5(6), 41-51.

De Paola, F., Fontana, N., Galdiero, E., Giugni, M., Savic, D., \& Uberti, G. S. (2014). Automatic Multi-objective Sectorization of a Water Distribution Network. Procedia Engineering, 89, 1200-1207. doi:10.1016/j.proeng.2014.11.250 
De Paola, F., Fontana, N., Galdiero, E., Giugni, M., Uberti, G. S., \& Vitaletti, M. (2014). Optimal Design of District Metered Areas in Water Distribution Networks. Procedia Engineering, 70, 449-457. doi:10.1016/j.proeng.2014.02.050

Deuerlein, J. (2008). Decomposition Model of a General Water Supply Network Graph. Journal of Hydraulic Engineering, 134(6), 822-832. doi:10.1061/(asce)07339429(2008)134:6(822)

Di Nardo, A., \& Di Natale, M. (2010). A Design Support Methodology for District Metering of Water Supply Networks. Water Distribution Systems Analysis 2010, 870-887. doi:10.1061/41203(425)80

Di Nardo, A., \& Di Natale, M. (2011). A heuristic design support methodology based on graph theory for district metering of water supply networks. Engineering Optimization, 43(2), 193-211. doi:10.1080/03052151003789858

Di Nardo, A., Cavallo, A., Di Natale, M., Greco, R., \& Santonastaso, G. F. (2016). Dynamic Control of Water Distribution System Based on Network Partitioning. Procedia Engineering, 154, 1275-1282. doi:10.1016/j.proeng.2016.07.460

Di Nardo, A., Cavallo, A., Di Natale, M., Greco, R., \& Santonastaso, G. F. (2016). Dynamic Control of Water Distribution System Based on Network Partitioning. Procedia Engineering, 154, 1275-1282. doi:10.1016/j.proeng.2016.07.460

Di Nardo, A., Di Natale, M., \& Di Mauro, A. (2013a). Water District Metering policy and practice. En Water Supply Network District Metering. Springer, Vienna: CISM International Centre for Mechanical Sciences. doi:10.1007/978-3-7091-1493-3_2

Di Nardo, A., Di Natale, M., Giudicianni, C., Santonastaso, G., Tzatchkov, V., \& Varela, J. (2017). Economic and Energy Criteria for District Meter Areas Design of Water Distribution Networks. Water, 9(7), 463. doi:10.3390/w9070463

Di Nardo, A., Di Natale, M., Greco, R., \& Santonastaso, G. F. (2014). Ant Algorithm for Smart Water Network Partitioning. Procedia Engineering, 70, 525-534. doi:10.1016/j.proeng.2014.02.058

Di Nardo, A., Di Natale, M., Musmarra, D., Santonastaso, G. F., Tuccinardi, F. P., \& Zaccone, G. (2015). Software for partitioning and protecting a water supply network. Civil Engineering and Environmental Systems, 33(1), 55-69. doi:10.1080/10286608.2015.1124867

Di Nardo, A., Di Natale, M., Musmarra, D., Santonastaso, G., Tzatchkov, V., \& AlcocerYamanaka, V. (2014). A District Sectorization for Water Network Protection from Intentional Contamination. Procedia Engineering, 70, 515-524. doi:10.1016/j.proeng.2014.02.057

Di Nardo, A., Di Natale, M., Santonastaso, G. F., \& Venticinque, S. (2011). Graph partitioning for automatic sectorization of a water distribution system. Proceedings of computer and control in water industry CCWI, 841-846.

Di Nardo, A., Di Natale, M., Santonastaso, G. F., Tzatchkov, V. G., \& Alcocer-Yamanaka, V. H. (2013b). Water network sectorization based on a genetic algorithm and minimum 
dissipated power paths. Water Science and Technology: Water Supply, 13(4), 951957. doi:10.2166/ws.2013.059

Di Nardo, A., Di Natale, M., Santonastaso, G., Tzatchkov, V. G., \& Alcocer- Yamanaka, V. (2014). Performance indices for water network partitioning and sectorization. Water Science and Technology: Water Supply, 15(3), 499-509. doi:10.2166/ws.2014.132

Diao, K., Sweetapple, C., Farmani, R., Fu, G., Ward, S., \& Butler, D. (2016). Global resilience analysis of water distribution systems. Water Research, 106, 383-393. doi:10.1016/j.watres.2016.10.011

Diao, K., Zhou, Y., \& Rauch, W. (2013). Automated Creation of District Metered Area Boundaries in Water Distribution Systems. Journal of Water Resources Planning and Management, 139(2), 184-190. doi:10.1061/(asce)wr.1943-5452.0000247

Dijkstra, E. W. (1959). A note on two problems in connexion with graphs. Numerische mathematik, 1(1), 269-271.

EPA. (2002). Effects of Water Age on Distribution System Water Quality. US Environmental Protection Agency, Washington, DC, USA.

EPA. (2010). Control and Mitigation of Drinking Water Losses in Distribution Systems. US Environmental Protection Agency, The Drinking Water Protection Division of the EPA Office of Ground Water and Drinking Water. Recuperado el 27 de enero de 2021, de https://nepis.epa.gov/Exe/ZyPURL.cgi?Dockey=P1009VCZ.txt

ERSAR, L. (2009). Guia de avaliação da qualidade dos serviços de águas e resíduos prestados aos utilizadores. Instituto Regulador de Águas e Resíduos (actual Entidade Reguladora de Sistemas de Águas e Resíduos) e Laboratório Nacional de Engenharia Civil.

Fang, D., \& Chen, B. (2017). Linkage analysis for the water-energy nexus of city. Applied Energy, 189, 770-779. doi:10.1016/j.apenergy.2016.04.020

Fanner, P., Davis, S., Hoogerwerf, T., Liemberger, R., Sturm, R., \& Thornton, J. (2007). Leakage management technologies. Awwa Research Foundation.

Farley, M. (1985). District metering. Part 1 - System design and installation. Swindon, U.K: WRc.

Farley, M. (2001). Leakage management and control: a best practice training manual. World Health Organization, Water, Sanitation and Health Team \& Water Supply and Sanitation Collaborative Council. doi:apps.who.int/iris/handle/10665/66893

Farley, M. (2008). The Manager's Non-Revenue Water Handbook - A Guide to Understanding Water Losses. IWA. Recuperado el 13 de febrero de 2021, de https://iwanetwork.org/learn_resources/the-managers-non-revenue-water-handbook/

Farley, M. (2010). Are there alternatives to the DMA? Asian Water, 26(10), 10-16.

Farley, M., \& Liemberger, R. (2005). Developing a non-revenue water reduction strategy: planning and implementing the strategy. Water Science and Technology: Water Supply, 5(1), 41-50. doi:10.2166/ws.2005.0006 
Farley, M., \& Trow, S. (2003). Losses in Water Distribution Networks. A Practitioner's Guide to Assessment, Monitoring and Control. London: IWA publishing.

Farmani, R., Wright, J., Savic, D., \& Walters, G. (2005). Selfadaptive fitness formulation for evolutionary constrained optimization of water systems. J. Comput. Civ. Eng., 19(2), 212-216. doi:10.1061/(ASCE)0887-3801(2005)19:2(212)

Fernández, M. F. (2012). Política y administración del abastecimiento de agua en Sevilla durante la Edad Moderna. Sevilla: Diputación de Sevilla, Servicio de Archivos y Publicaciones.

Ferrari, G., \& Savic, D. (2015). Economic Performance of DMAs in Water Distribution Systems. Procedia Engineering, 119, 189-195. doi:10.1016/j.proeng.2015.08.874

Ferrari, G., \& Savic, D. (2015). Economic Performance of DMAs in Water Distribution Systems. Procedia Engineering, 119, 189-195. doi:10.1016/j.proeng.2015.08.874

Ferrari, G., Savic, D., \& Becciu, G. (2014). Graph-Theoretic Approach and Sound Engineering Principles for Design of District Metered Areas. Journal of Water Resources Planning and Management, 140(12). doi:10.1061/(asce)wr.1943-5452.0000424

Filion, Y. R., MacLean, H. L., \& Karney, B. W. (2004). Life-Cycle Energy Analysis of a Water Distribution System. Journal of Infrastructure Systems, 10(3), 120-130. doi:10.1061/(asce)1076-0342(2004)10:3(119)

Filion, Y. R., MacLean, H. L., \& Karney, B. W. (2004). Life-Cycle Energy Analysis of a Water Distribution System. Journal of Infrastructure Systems, 10(3), 120-130. doi:10.1061/(asce)1076-0342(2004)10:3(119)

Fu, G., Kapelan, Z., \& Reed, P. (2012). Reducing the Complexity of Multiobjective Water Distribution System Optimization through Global Sensitivity Analysis. Journal of Water Resources Planning and Management, 138(3), 196-207. doi:10.1061/(asce)wr.194354

Fuchs-Hanusch, D., \& Steffelbauer, D. (2017). Real-world Comparison of Sensor Placement Algorithms for Leakage Localization. Procedia Engineering, 186, 499-505. doi:10.1016/j.proeng.2017.03.262

Galdiero, E., De Paola, F., Fontana, N., Giugni, M., \& Savic, D. (2016). Decision support system for the optimal design of district metered areas. J. Hydroinf, 18(1), 49-61. doi:10.2166/hydro.2015.023

Gamboa-Medina, M. M., \& Reis, L. F. (2017). Sampling Design for Leak Detection in Water Distribution Networks. Procedia Engineering, 186, 460-469. doi:10.1016/j.proeng.2017.03.255

Gheisi, A., \& Naser, G. (2015). Multistate Reliability of Water-Distribution Systems: Comparison of Surrogate Measures. Journal of Water Resources Planning and Management, 141(10), 04015018. doi:10.1061/(asce)wr.1943-5452.0000529

Gheisi, A., Forsyth, M., \& Naser, G. (2016). Water Distribution Systems Reliability: A Review of Research Literature. Journal of Water Resources Planning and Management, 142(11), 04016047. doi:10.1061/(asce)wr.1943-5452.0000690 
Girvan, M., \& Newman, M. (2002). Community structure in social and biological networks. Proceedings of the National Academy of Sciences, 99(12), 7821-7826. doi:10.1073/pnas.122653799

Giustolisi, O., \& Ridolfi, L. (2014). Modularity Index for Hydraulic System Segmentation. Procedia Engineering, 89, 1152-1159. doi:10.1016/j.proeng.2014.11.240

Giustolisi, O., Ridolfi, L., \& Berardi, L. (2015). General metrics for segmenting infrastructure networks. Journal of Hydroinformatics, 17(4), 505-517. doi:10.2166/hydro.2015.102

GIZ, \& VAG. (2011). Guía para la reducción de las pérdidas de agua: un enfoque en la gestión de la presión. Deutsche Gesellschaft für Internationale Zusammenarbeit (GIZ) GmbH y VAG-Armaturen $\mathrm{GmbH}$, Alemania. Recuperado el 1 de abril de 2016, de http://www.waterloss-reduction.com

Global Omnium. (2019). Informe de Sostenibilidad. Recuperado el 9 de mayo de 2021, de https://www.globalomnium.com/Sites/20/Docs/Sostenibilidad/GO_EINOF_GRI_2019. pdf

Gomes, R., Marques, A. S., \& Sousa, J. (2012). Decision support system to divide a large network into suitable District Metered Areas. Water Science and Technology, 65(9), 1667-1675. doi:10.2166/wst.2012.061

Gomes, R., Marques, A., \& Sousa, J. (2013). District Metered Areas Design Under Different Decision Makers' Options: Cost Analysis. Water Resources Management,, 27(13), 4527-4543. doi:10.1007/s11269-013-0424-y

Gómez, E. (2016). Caracterización y mejora de la eficiencia energética del transporte de agua a presión. Doctoral dissertation, Universitat Politècnica de València. doi:10.4995/Thesis/10251/72637

Gong, W., Suresh, M. A., Smith, L., Ostfeld, A., Stoleru, R., Rasekh, A., \& Banks, M. K. (2016). Mobile sensor networks for optimal leak and backflow detection and localization in municipal water networks. Environmental Modelling \& Software, 80, 306-321. doi:10.1016/j.envsoft.2016.02.001

Grayman, Murray, R., Savic, A., \& Farmani, R. (2016). Redesign of water distribution systems for passive containment of contamination. Journal-American Water Works Association(108), 381-391. doi:10.5942/jawwa.2016.108.0105

Grayman, W., Murray, R., \& Savic, D. (2009). Effects of Redesign of Water Systems for Security and Water Quality Factors. World Environmental and Water Resources Congress 2009. doi:10.1061/41036(342)49

Grayman, W., Murray, R., Savic, D., \& Farmani, R. (2016). Redesign of water distribution systems for passive containment of contamination. Journal-American Water Works Association, 108(7), 381-391.

Hardy, L., Garrido, A., \& Juana, L. (2012). Evaluation of Spain's Water-Energy Nexus. International Journal of Water Resources Development, 28(1), 151-170. doi:10.1080/07900627.2012.642240 
Herrera, A. M., Gutiérrez-Pérez, J. A., Izquierdo, J., \& Pérez, R. (2012). Combining multiple perspectives on clustering. Node-pipe case in hydraulic sectorization. International Journal of Complex Systems in Science, 2(1), 17-2.

Herrera, M., Abraham, E., \& Stoianov, I. (2016a). A Graph-Theoretic Framework for Assessing the Resilience of Sectorised Water Distribution Networks. Water Resources Management, 30(5), 1685-1699. doi:10.1007/s11269-016-1245-6

Herrera, M., Izquierdo, J., Pérez-García, R., \& Ayala-Cabrera, D. (2010). Water supply clusters by multi-agent based approach. Water distribution systems analysis 2010, 861-869.

Herrera, M., Wright, R., Abraham, E., Izquierdo, J., \& Perez-Garcia, R. (2016b). Condiciones hidráulicas sobre medidas de centralidad en grafos para la evaluación de la resiliencia de redes de distribución de agua. Acta Universitaria, 26(3), 82-90. doi:10.15174/au.2016.1058

Huang, G. (2003). Casting the Wireless Sensor Net. MIT's Magazine of Innovation, 51-56.

Hunaidi, O. (2005). Economic comparison of periodic acoustic surveys and DMA-based leakage management strategies. Leakage 2005 - Conference Proceedings Benchmarking the Water Losses.

INE. (2018). Encuesta sobre el suministro y saneamiento del agua. Recuperado el 14 de marzo de 2019, de http://www.ine.es/dyngs/INEbase/es/operacion.htm?c=Estadistica_C\&cid=125473617 $6834 \&$ menu $=u l t i D a t o s \& i d p=1254735976602$

Instituto Aragonés del Agua. (2013). Tarifas del agua. Obtenido de https://www.aragon.es/documents/20127/674325/Tarifas\%20de\%20Agua_09_2015.p df/a7562776-5ba5-5116-5e6d-ce8b0d842aac

Kingdom, B., Liemberger, R., \& Marin, P. (2006). The Challenge of Reducing Non-Revenue Water in Developing Countries - How the Private Sector Can Help: A Look at Performance-Based Service Contracting. Water Supply and Sanitation Sector Board discussion paper series; n‥ 8, World Bank, Washington, DC. Recuperado el 27 de enero de 2021, de https://openknowledge.worldbank.org/handle/10986/17238

Korkana, P., Kanakoudis, V., Makrysopoulos, A., Patelis, M., \& Gonelas, K. (2016). Developing an Optimization Algorithm to form District Metered Areas in a Water Distribution System. Procedia Engineering. Procedia Engineering, 162, 530-536. doi:10.1016/j.proeng.2016.11.097

Lai, T. T., Chen, Y. H., Huang, P., \& Chu, H. H. (2010). PipeProbe: a mobile sensor droplet for mapping hidden pipeline. In Proceedings of the 8th ACM Conference on Embedded Networked Sensor Systems, 113-126. doi:10.1145/1869983.1869996

Lambert, A., \& Hirner, W. (2000). Losses from Water Supply Systems: Standard Terminology and Recommended Performance. International Water Association.

Laucelli, D. B., Simone, A., Berardi, L., \& Giustolisi, O. (2017). Optimal Design of District Metering Areas for the Reduction of Leakages. Journal of Water Resources Planning and Management, 143(6), 04017017. doi:10.1061/(asce)wr.1943-5452.0000768 
Lee, M., Keller, A. A., Chiang, P.-C., Den, W., Wang, H., Hou, C.-H., \& Yan, J. (2017). Waterenergy nexus for urban water systems: A comparative review on energy intensity and environmental impacts in relation to global water risks. Applied Energy, 589-601. doi:10.1016/j.apenergy.2017.08.002

Lence, B. J., Moosavian, N., \& Daliri, H. (2017). Fuzzy Programming Approach for Multiobjective Optimization of Water Distribution Systems. Journal of Water Resources Planning and Management, 143(7). doi:10.1061/(asce)wr.1943-5452.0000769

Liemberger, R., \& Farley, M. (2005). Developing a nonrevenue water reduction strategy Part 1: Investigating and assessing water losses. Water Science and Technology: Water Supply, 5(1), 41-50. doi:10.2166/ws.2005.0006

Lopes, R. E., \& Martinez, C. B. (2006). Use of pumps as turbines for systems with water discharge. Proceedings of VISEREA-Seminário Iberoamericano sobre Sistemas de Abastecimento Urbano de Água, João Pessoa, Brazil.

MacDonald, G., \& Yates, C. D. (2005). DMA design and implementation, a North American context. Leakage Conference. IWA.

Martin, D. W., \& Peters, G. (1963). The Application of Newton's Method to Network Analysis by Digital Computer. Journal ofthe Institution of Water Engineers , 66.

Ministerio de Medio Ambiente. (2007). Precios y costes de los Servicios del Agua en España Informe integrado de recuperación de costes de los servicios de agua en España. España. http://hispagua.cedex.es/sites/default/files/especiales/Tarifas_agua/precios_. Recuperado el 19 de octubre de 2019, de http://hispagua.cedex.es/sites/default/files/especiales/Tarifas_agua/precios_costes_s ervicios_\%20agua.pdf

Möderl, M., Hellbach, C., Sitzenfrei, R., Mair, M., Lukas, A., Mayr, E., \& Rauch, W. (2011). GIS based applications of sensitivity analysis for water distribution models. In World Environmental and Water Resources Congress 2011: Bearing Knowledge for Sust. In World Environmental and Water Resources Congress 2011: Bearing Knowledge for Sustainability, 129-136. doi:10.1061/41173(414)14

Monsef, H., Naghashzadegan, M., Farmani, R., \& Jamali, A. (2019). Deficiency of reliability indicators in water distribution networks. Journal of Water Resources Planning and Management, 145(6). doi:10.1061/(ASCE)WR.1943-5452.0001053

Morris Jr, R. (1967). Principal causes and remedies of water main breaks. Journal American Water Works Association, 59(7), 782-798.

Morrison, J., Tooms, S., \& Rogers, D. (2007). District metered areas: Guidance notes. IWA, Water Loss Task Force.

Newman, M. (2004). Detecting community structure in networks. The European Physical Journal B - Condensed Matter, 38(2), 321-330. doi:10.1140/epjb/e2004-00124-y

Newman, M., \& Girvan, M. (2003). Mixing Patterns and Community Structure in Networks. Lecture Notes in Physics, 66-87. doi:10.1007/978-3-540-44943-0_5 
OECD. (2012). Environmental Outlook to 2050: The Consequences of Inaction. Organización para la Cooperación y Desarrollo Económicos. París: OECD.

OECD. (2012). Environmental Outlook to 2050: The Consequences of Inaction. Organización para la Cooperación y Desarrollo Económicos. París: OCDE. doi:10.1787/1999155x

Ofwat. (2008). International comparison - leakage. Obtenido de http://www.ofwat.gov.uk/regulating/reporting/

OIT. (2013). El desarrollo sostenible, el trabajo decente y los empleos verdes. Organización Internacional del Trabajo, Informe No. 5, Conferencia Internacional del Trabajo, 102 reunión. Ginebra, Suiza: OIT.

OIT. (2015). Perspectivas sociales y del empleo en el mundo - Tendencias 2015. Organización Internacional del Trabajo. Ginebra, Suiza: OIT.

Ortí, R. B., Toda, A. C., \& Val, A. A. (2008). Los costes de los servicios urbanos del agua. Un análisis necesario para el establecimiento y control de tarifas. Hacienda pública española, 186, 123-155.

Ostfeld, A., Kogan, D., \& Shamir, U. (2002). Reliability simulation of water distribution systems - Single and multiquality. Urban Water, 4(1), 53-61. doi:10.1016/S14620758(01)00055-

Özdemir, Ö. (2018). Water leakage management by district metered areas at water distribution networks. Environmental Monitoring and Assessment, 190(4). doi:10.1007/s10661018-6559-9

Pelli, T., \& Hitz, H. U. (2000). Energy indicators and savings in water supply. Journal-American Water Works Association, 92(6), 55-62. doi:10.2307/41297339

Perelman, L. S., Allen, M., Preis, A., Iqbal, M., \& Whittle, A. J. (2014). Multi-level automated sub-zoning of water distribution systems. Proceedings of the 7th International Congress on Environmental Modelling and Software.

Perelman, L., \& Ostfeld, A. (2011). Topological clustering for water distribution systems analysi. Environmental Modelling \& Software, 26(7), 969-972. doi:10.1016/j.envsoft.2011.01.006

Perelman, L., Allen, M., Preis, A., Iqbal, M., \& Whittle, A. (2015). Automated sub-zoning of water distribution systems. Environmental Modeling \& Software, 65, 1-14. doi:10.1016/j.envsoft.2014.11.025

Pereyra, G., Pandolfi, D. R., \& Villagra, N. A. (2017). Diseño y optimización de redes de distribución de agua utilizando algoritmos genéticos. Informe Científico Técnico UNPA, 9(1), 37-63.

Pérez, R. (1993). Dimensionamiento óptimo de redes de distribución de agua ramificadas considerando los elementos de regulación. Tesis doctoral. Valencia: Universidad Politécnica de Valencia. Director: Fernando Martínez Alzamora.

Pérez, R., Puig, V., Pascual, J., Quevedo, J., Landeros, E., \& Peralta, A. (2011). Methodology for leakage isolation using pressure sensitivity analysis in water distribution networks. 
Control Engineering Practice, $\quad 19(10), \quad$ 1157-1167. doi:10.1016/j.conengprac.2011.06.004

Pesantez, J. E., Berglund, E. Z., \& Mahinthakumar, G. (2019). Multiphase Procedure to Design District Metered Areas for Water Distribution Networks. Journal of Water Resources Planning and Management, 145(8), 04019031. doi:10.1061/(asce)wr.19435452.0001095

Pesantez, J. E., Berglund, E. Z., \& Mahinthakumar, G. (2019). Multiphase Procedure to Design District Metered Areas for Water Distribution Networks. Journal of Water Resources Planning and Management, 145(8). doi:10.1061/(asce)wr.1943-5452.00010

Pilcher, R. (2003). Leak Detection Practices and Techniques: A Practical Approach. Water21. IWA Water Loss Task Force, Diciembre, 44-45.

Prasad, T. D., \& Park, N. S. (2004). Multiobjective Genetic Algorithms for Design of Water Distribution Networks. Journal of Water Resources Planning and Management, 130(1), 73-82. doi:10.1061/(asce)0733-9496(2004)130:1(73)

Prasad, T. D., \& Tanyimboh, T. T. (2008). Entropy Based Design of Anytown" Water Distribution Network. Proceedings of the 10th Annual Water Distribution Systems Analysis Conference WDSA2008. doi:10.1061/41024(340)39

Prim, R. C. (1957). Shortest connection networks and some generalizations. The Bell System Technical Journal, 36(6), 1389-1401.

Pudar, R. S., \& Liggett, J. A. (1992). Leaks in Pipe Networks. Journal of Hydraulic Engineering, 118(7), 1031-1046. doi:10.1061/(asce)0733-9429(1992)118:7(1031)

Puust, R., Kapelan, Z., Savic, D. A., \& Koppel, T. (2010). A review of methods for leakage management in pipe networks. Urban Water Journal, 7(1), 25-45. doi:10.1080/15730621003610878

Puust, R., Kapelan, Z., Savic, D., \& Koppel, T. (2010). A review of methods for leakage management in pipe networks. Urban Water Journal, 7(1), 25-45. doi:10.1080/15730621003610878

Ramírez, L. G., Jiménez, G. S., \& Carreño, J. M. (2014). Sensores y actuadores. Grupo Editorial Patria.

Renaud, E.; Pillot, J.; Aubrun, C.; Bréjoux, E.; Augeard, B. (2107). Water loss reduction: a new approach to achieve real water saving and environmental benefits. Water Science and Technology: Water Supply, 18(1), 251-258. doi:10.2166/ws.2017.099

Rossman, L. A. (Septiembre de 2002). EPANET 2 Users Manual. US Environmental Protection Agency, National Risk Management Research Laboratory, Cincinnati, Ohio.

Saldarriaga, J. (2007). Hidráulica de Tuberías: Abastecimiento de Agua, Redes y Riego. Marcombo.

Saldarriaga, J. (2016). Battle of water networks DMAs. Journal of Water Resources Planning and Management, 140(7). doi:10.1061/(ASCE)WR.1943-5452.0000830 
Salguero, F. J., Cobacho, R., \& Pardo, M. A. (2018). Unreported leaks location using pressure and flow sensitivity in water distribution networks. Water Science and Technology: Water Supply, ws2018048. doi:10.2166/ws.2018.048

Sarbu, I. (2009). Design of optimal water distribution systems. International journal of energy, $4(3), 59-67$.

Sarrate, R., Blesa, J., Nejjari, F., \& Quevedo, J. (2014). Sensor placement for leak detection and location in water distribution networks. Water Science and Technology: Water Supply, 14(5), 795-803. doi: 10.2166/ws.2014.037. Water Science and Technology: Water Supply, 14(5), 795-803. doi:10.2166/ws.2014.037

Savić, D., \& Ferrari, G. (2014). Design and Performance of District Metering Areas in Water Distribution Systems. Procedia Engineering, 89, 1136-1143. doi:10.1016/j.proeng.2014.11.236

Scarpa, F., Lobba, A., \& Becciu, G. (2016). Elementary DMA Design of Looped Water Distribution Networks with Multiple Sources. Journal of Water Resources Planning and Management, 142(6). doi:10.1061/(asce)wr.1943-5452.0000639

Schaeffer, S. E. (2007). Graph clustering. Computer Science Review. Computer Science Review, 1(1), 27-64. doi:10.1016/j.cosrev.2007.05.001

Scibetta, M., Boano, F., Revelli, R., \& Ridolfi, L. (2014). Community Detection as a Tool for District Metered Areas Identification. Procedia Engineering, 70, 1518-1523. doi:10.1016/j.proeng.2014.02.167

Scott, C. A., Pierce, S. A., Pasqualetti, M. J., Jones, A. L., Montz, B. E., \& Hoover, J. H. (2011). Policy and institutional dimensions of the water-energy nexus. Energy Policy, 39(10), 6622-6630. doi:10.1016/j.enpol.2011.08.013

Shannon, C. (1948). A mathematical theory of communication. Bell System Tech. J., 27, 379423.

Sharif, M. N., Haider, H., Farahat, A., Hewage, K., \& Sadiq, R. (2019). Water Energy Nexus for Water Distribution Systems: A Literature Review. Environmental Reviews. doi:10.1139/er-2018-0106

Siegel, S. (2017). Hágase el agua: La Solución de Israel para un mundo hambriento de agua. Nagrela.

Smith, K., Liu, S., Liu, Y., Savic, D., Olsson, G., Chang, T., \& Wu, X. (2015). Impact of urban water supply on energy use in China: a provincial and national comparison. Mitigation and Adaptation Strategies for Global Change, 21(8), 1213-1233. doi:10.1007/s11027015-9648-x

Steffelbauer, D., Neumayer, M., Günther, M., \& Fuchs-Hanusch, D. (2014). Sensor placement and leakage localization considering demand uncertainties. Procedia Engineering, 89, 1160-1167. doi:10.1016/j.proeng.2014.11.242

Stephens, M., Gong, J., Zhang, C., Marchi, A., Dix, L., \& Lambert, M. (2020). Leak-BeforeBreak Main Failure Prevention for Water Distribution Pipes Using Acoustic Smart Water 
Technologies: Case Study in Adelaide. Journal of Water Resources Planning and Management, 146(10). doi:10.1061/(ASCE)WR.1943-5452.0001266

Sturm, M., \& Thornton, J. (2005). Proactive Leakage Management using DMA and Pressure Management - Is it applicable in North America. In IWA Specialised Conference “Leakage 2005”, Conference Proceedings, p426.

Sturm, R., \& Thornton, J. (2005). Proactive leakage management using District Metered Areas (DMA) and pressure management-Is it applicable in North America. In Leakage 2005 Conference, Canada.

Tanyimboh, T. T., \& Templeman, A. B. (1993). Optimum design of flexible water distribution networks. Civil Engineering Systems, 10(3), 243-258. doi:10.1080/02630259308970126

Tanyimboh, T. T., \& Templeman, A. B. (2000). A quantified assessment of the relationship between the reliability and entropy of water distribution systems. Eng. Optim, 33(2), 179-199. doi:10.1080/03052150008940916.

Thornton, J. (October de 2003). Managing leakage by managing pressure: a practical approach. Water 21, 43-44.

Todini, E. (2000). Looped water distribution networks design using a resilience index based heuristic approach. Urban Water, 2(2), 115-122. doi:10.1016/s1462-0758(00)00049-2

Todini, E., \& Pilati, S. (1987). A gradient method for the analysis of pipe networks. In International Conference on Computer Applications for Water Supply and Distribution, Leicester Polytechnic, UK.

Todini, E., \& Pilati, S. (1988). A gradient method for the solution of looped pipe networks. Computer applications in water supply, 1, 1-20.

Todini, E., \& Rossman, L. A. (2013). Unified framework for deriving simultaneous equation algorithms for water distribution networks. Journal of Hydraulic Engineering, 139(5), 511-526. doi:10.1061/(ASCE)HY.1943-7900.0000703

Tzatchkov, V. (2007). Datos básicos. 3-ra edición, Manuales de Diseño de Agua Potable.

Tzatchkov, V. G., Alcocer-Yamanaka, V. H., \& Bourguett Ortíz, V. (2008). Graph Theory Based Algorithms for Water Distribution Network Sectorization Projects. Water Distribution Systems Analysis Symposium. doi:10.1061/40941(247)172

Tzatchkov, V. G., Alcocer-Yamanaka, V. H., \& Ortiz, V. B. (2012). Graph partitiioning algorithms for water distribution network sectorization projects. In Proceedings of 10th international conference on hydroinformatics HIC.

Tzatchkov, V., Alcocer, V., \& Rodriguez-Varela, J. (2006). Water Distribution Network Sectorization Projects in Mexican Cities along the Border with USA. Proceedings of the 3rd International Symposium on Transboundary Water Management, 1-13.

Tzatchkov, V., Alcocer, V., Bourguett, V., \& Arreguín, F. (2014). Avances en la hidráulica de redes de distribución de agua potable. Jiutepec, Mor: Instituto Mexicano de Tecnología del Agua. 
UNESCO. (2017). Resumen del Informe de seguimiento de la educación en el mundo, 2017/8. Rendir cuentas en el ámbito de la educación:: Organización de las Naciones Unidas para la Educación, la Ciencia y la Cultura, París. Obtenido de unesdoc.unesco.org/images/0025/002593/259338e.pdf

United Utilities Water plc. (2015). Design guidance for water mains and services on new development sites. Document Ref. 20368. Issue 3 November 2015.

Vakilifard, N., Bahri, P. A., Anda, M., \& Ho, G. (2017). Water Security and Clean Energy, Cobenefits of an Integrated Water and Energy Management. 27th European Symposium on Computer Aided Process Engineering, 1363-1368. doi:10.1016/b978-0-444-639653.50

Vegas, O., Martínez, F., \& Tzatchkov, V. (2016). Aplicación de la teoría de grafos a la identificación de subsistemashidráulicos en redes de distribución de agua. XXVII CongresoLatinoamericano de Hidráulica.

Viollet, P. (2010). La ingeniería y la gestión del agua en las primeras civilizaciones en la Edad de Bronce. En E. Cabrera Jr., La ingeniería y la gestión del agua a través de los tiempos (págs. 45-76). Valencia: Cabrera y Arregui.

Water Authorities Association. (1985). Leakage Control Policy and Practice. London.

Watson, K., Klingenberg, B., Polito, T., \& Geurts, T. (2004). Impact of environmental management system implementation on financial performance: A comparison of two corporate strategies. Management of Environmental Quality, 15(6), 622-628. doi:10.1108/14777830410560700

Wojciech, K., \& Krzysztof, D. (2012). Sectorisation, reduction and stabilisation of pressure as a key to control and decrease of leakage. Case study from Bytom, Poland. Water Loss 2012. Manila, Philipinnes.

Wong, L., Deo, R., Rathnayaka, S., Shannon, B., Zhang, C., Chiu, W., \& Widyastuti, H. (2018). Leak Detection in Water Pipes Using Submersible Optical Optic-Based Pressure Sensor. Sensors, 18(12), 4192. doi:10.3390/s18124192

Wood, D. J., \& Charles, O. A. (1972). Hydraulic Network Analysis Using Linear Theory. Journal of the Hydraulics División, ASCE , 98.

Wright, R., Abraham, E., Parpas, P., \& Stoianov, I. (2015). Optimized Control of Pressure Reducing Valves in Water Distribution Networks with Dynamic Topology. Procedia Engineering. Procedia doi:10.1016/j.proeng.2015.08.994

Engineering(119), 1003-1011.

Wu, W., Dandy, G. C., \& Maier, H. R. (2014). Protocol for developing ANN models and its application to the assessment of the quality of the ANN model development process in drinking water quality modelling. Environmental Modelling \& Software, 54, 108-127. doi:10.1016/j.envsoft.2013.12.016

WWAP (United Nations World Water Assessment Program). (2018). UN World Water Development Report, Nature-based Solutions for Water. Paris: UNESCO. 
WWAP. (2012). The United Nations World Water Development Report 4: Managing Water under Uncertainty and Risk. Programa Mundial de Evaluación de los Recursos Hídricos. París: UNESCO.

WWAP. (2014). The United Nations World Water Development Report 2014: Water and Energy. Programa Mundial de Evaluación de los Recursos. París: UNESCO.

WWAP. (2016). Desarrollo de los Recursos Hídricos en el Mundo 2016. UNESCO, Programa Mundial de Evaluación de los Recursos Hídricos, París.

WWAP. (2019). Informe Mundial de las Naciones Unidas sobre el Desarrollo de los Recursos Hidricos 2019: No dejar a nadie atrás. UNESCO, Programa Mundial de Evaluación de los Recursos Hídricos de la UNESCO, París.

Xie, X., Zhou, Q., Hou, D., \& Zhang, H. (2017). Compressed sensing based optimal sensor placement for leak localization in water distribution networks. Journal of Hydroinformatics, jh2017145. doi:10.2166/hydro.2017.145

Yazdani, A., \& Jeffrey, P. (2011). Complex network analysis of water distribution systems. Chaos: An Interdisciplinary Journal of Nonlinear Science, 21(1), 016111. doi:10.1063/1.3540339

Yazdani, A., \& Jeffrey, P. (2012). Water distribution system vulnerability analysis using weighted and directed network models. Water Resources Research, 48(6). doi:10.1029/2012wr011897

Zhang, Q., Wu, Z. Y., Zhao, M., Qi, J., Huang, Y., \& Zhao, H. (2017). Automatic Partitioning of Water Distribution Networks Using Multiscale Community Detection and Multiobjective Optimization. Journal of Water Resources Planning and Management, 143(9), 04017057. doi:10.1061/(asce)wr.1943-5452.0000819

Zheng, F., Simpson, A. R., \& Zecchin, A. C. (2011). A combined NLP-differential evolution algorithm approach for the optimization of looped water distribution systems. Water Resources Research, 47(8). doi:10.1029/2011wr010394 